\title{
Synthesis of 1H-pyrazol-5-yl-pyridin-2-yl-[1,2,4]triazinyl Soft-Lewis Basic Complexants via Metal and Oxidant Free [3+2] Dipolar Cycloaddition of Terminal Ethynyl Pyridines with Tosylhydrazides
}

Giri Babu Veerakanellore, ${ }^{1}$ Caris M. Smith, ${ }^{2}$ Monica Vasiliu, ${ }^{2}$ Allen G. Oliver, ${ }^{3}$ David A. Dixon, ${ }^{2}$ and Jesse D. Carrick*1

${ }^{1}$ Department of Chemistry, Tennessee Technological University, 55 University Drive, Cookeville, TN 38505-0001

${ }^{2}$ Department of Chemistry, The University of Alabama, Box 870336, Tuscaloosa, AL 35487-0336

${ }^{3}$ Department of Chemistry, The University of Notre Dame, 236 Nieuwland Science Hall, Notre Dame, IN 46556

Department of Chemistry, Tennessee Technological University, 55 University Drive, Cookeville, TN 38505-0001

Table of Contents

S1-S4

H1 N'-(4-methylbenzylidene)-4-methylbenzenesulfonohydrazide ${ }^{1} \mathrm{H}$ NMR

S5

H1 N'-(4-methylbenzylidene)-4-methylbenzenesulfonohydrazide ${ }^{13} \mathrm{C}$ NMR

H2 N'-(N,N-dimethylaminobenzylidene)-4-methylbenzenesulfono-hydrazide ${ }^{1} \mathrm{H}$ NMR

H2 N'-(N,N-dimethylaminobenzylidene)-4-methylbenzenesulfono-hydrazide ${ }^{13} \mathrm{C}$ NMR

S8

H3 N'-(2-iodobenzylidene)-4-methylbenzenesulfonohydrazide ${ }^{1} \mathrm{H}$ NMR

H3 N'-(2-iodobenzylidene)-4-methylbenzenesulfonohydrazide ${ }^{13} \mathrm{C}$ NMR

H4 N'-(3,5-di-tert-butylbenzylidene)-4-methylbenzenesulfono-hydrazide ${ }^{1} \mathrm{H} N \mathrm{NR}$

H4 N'-(3,5-di-tert-butylbenzylidene)-4-methylbenzenesulfono-hydrazide ${ }^{13} \mathrm{C}$ NMR

H5 N'-(pyridin-4-yl-benzylidene)-4-methylbenzenesulfono-hydrazide ${ }^{1} \mathrm{H} N \mathrm{NR}$

S13

H5 N'-(pyridin-4-yl-benzylidene)-4-methylbenzenesulfono-hydrazide ${ }^{13} \mathrm{C}$ NMR

H6 N'-(4-tert-butylbenzylidene)-4-methylbenzenesulfonohydrazide ${ }^{1} \mathrm{H} N \mathrm{NR}$

H6 N'-(4-tert-butylbenzylidene)-4-methylbenzenesulfonohydrazide ${ }^{13} \mathrm{C}$ NMR

H7 N'-(2,4,6-trimethoxybenzylidene)-4-methylbenzenesulfono-hydrazide ${ }^{1} \mathrm{H} N \mathrm{NR}$

H7 N'-(2,4,6-trimethoxybenzylidene)-4-methylbenzenesulfono-hydrazide ${ }^{13} \mathrm{C}$ NMR $\quad$ S18

H8 N'-(C-6-bromo-pyridin-2-yl-benzylidene)-4-methylbenzenesulfono-hydrazide ${ }^{1} \mathrm{H}$ NMR S19

H8 N'-(C-6-bromo-pyridin-2-yl-benzylidene)-4-methylbenzenesulfono-hydrazide ${ }^{13} \mathrm{C}$ NMR S20

H9 N'-(2-bromo-4-tert-butyl-6-phenol benzylidene)-4-methyl-benzenesulfono-hydrazide

${ }^{1} \mathrm{H}$ NMR

H9 N'-(2-bromo-4-tert-butyl-6-phenol benzylidene)-4-methyl-benzenesulfono-

Hydrazide ${ }^{13} \mathrm{C}$ NMR

Compound $1{ }^{1} \mathrm{H}$ NMR

Compound $1{ }^{13} \mathrm{C}$ NMR

S24

Compound $4{ }^{1} \mathrm{H}$ NMR

S25

Compound $4{ }^{13} \mathrm{C}$ NMR

S26

Compound $5^{1} \mathrm{H}$ NMR

Compound $5{ }^{13} \mathrm{C}$ NMR

Compound $6{ }^{1} \mathrm{H}$ NMR

S29

Compound $6{ }^{13} \mathrm{C}$ NMR

S30

Compound $7{ }^{1} \mathrm{H}$ NMR

S31 
Table of Contents, cont.

Compound $7{ }^{13} \mathrm{C}$ NMR $\quad$ S32

Compound $8{ }^{1} \mathrm{H}$ NMR

Compound $8{ }^{13} \mathrm{C}$ NMR $\quad$ S34

Compound $9{ }^{1} \mathrm{H}$ NMR $\quad$ S35

Compound $9{ }^{13} \mathrm{C}$ NMR $\quad$ S36

$\begin{array}{ll}\text { Compound } 2 \text { Major regioisomer }{ }^{1} \mathrm{H} \text { NMR } & \text { S37 }\end{array}$

$\begin{array}{ll}\text { Compound } 2 \text { Major regioisomer }{ }^{13} \mathrm{C} \text { NMR } & \text { S38 }\end{array}$

Compound 3 Minor regioisomer ${ }^{1} \mathrm{H}$ NMR $\quad$ S39

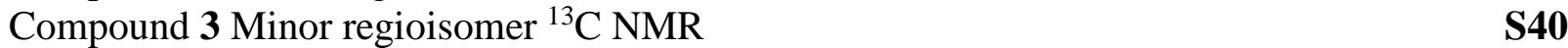

Compound $10^{1} \mathrm{H}$ NMR $\quad$ S41

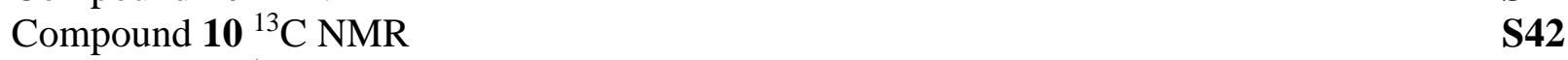

Compound $11^{1} \mathrm{H}$ NMR $\quad$ S43

Compound $11{ }^{13} \mathrm{C}$ NMR $\quad$ S44

Compound $12^{1} \mathrm{H}$ NMR $\quad$ S45

Compound $12{ }^{13} \mathrm{C}$ NMR $\quad$ S46

$\begin{array}{lr}\text { Compound } 13^{1} \mathrm{H} \text { NMR } & \text { S47 }\end{array}$

Compound $13{ }^{13} \mathrm{C}$ NMR $\quad$ S48

Compound $14^{1} \mathrm{H}$ NMR

Compound $14{ }^{13} \mathrm{C}$ NMR $\quad$ S50

Compound $15{ }^{1} \mathrm{H}$ NMR $\quad$ S51

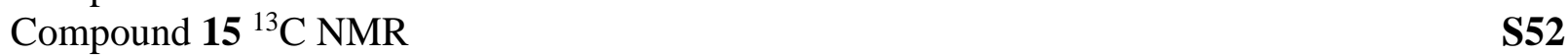

Compound $16{ }^{1} \mathrm{H}$ NMR $\quad$ S53

Compound $16{ }^{13} \mathrm{C}$ NMR $\quad$ S54

Compound $17{ }^{1} \mathrm{H}$ NMR $\quad S 55$

Compound $17^{13} \mathrm{C}$ NMR $\quad$ S56

$\begin{array}{ll}\text { Compound } 18{ }^{1} \mathrm{H} \text { NMR } & \text { S57 }\end{array}$

Compound $18{ }^{13} \mathrm{C}$ NMR $\quad$ S58

Compound $19^{1} \mathrm{H}$ NMR $\quad$ S59

Compound $19{ }^{13} \mathrm{C}$ NMR $\quad$ S60

Compound $20^{1} \mathrm{H}$ NMR $\quad$ S61

Compound $20^{13} \mathrm{C}$ NMR $\quad$ S62

Compound $21{ }^{1} \mathrm{H}$ NMR $\quad$ S63

Compound $21{ }^{13} \mathrm{C}$ NMR $\quad$ S64

Compound $22{ }^{1} \mathrm{H}$ NMR $\quad$ S65

Compound $22{ }^{13} \mathrm{C}$ NMR $\quad$ S66

Compound $23^{1} \mathrm{H}$ NMR $\quad$ S67

Compound $23{ }^{13} \mathrm{C}$ NMR $\quad$ S68

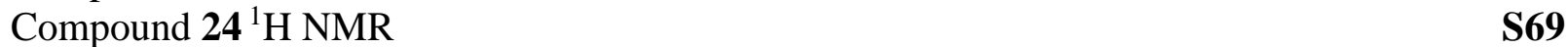

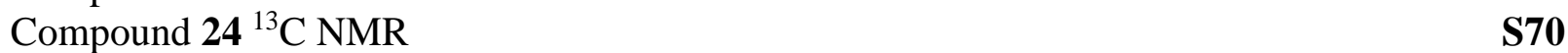

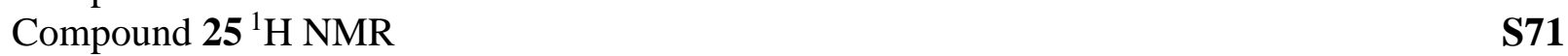

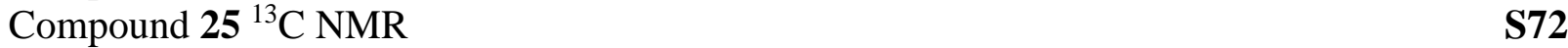

Compound $26{ }^{1} \mathrm{H}$ NMR $\quad$ S73

Compound $26{ }^{13} \mathrm{C}$ NMR $\quad$ S74

Compound $27^{1} \mathrm{H}$ NMR $\quad \mathbf{S 7 5}$ 
Table of Contents, cont.

Compound $27{ }^{13} \mathrm{C}$ NMR $\quad$ S76

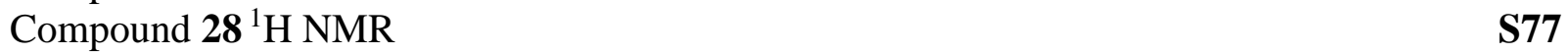

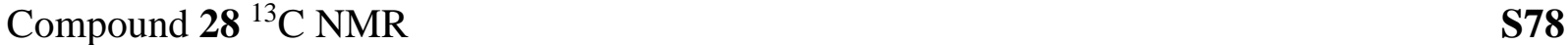

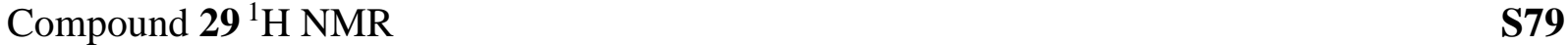

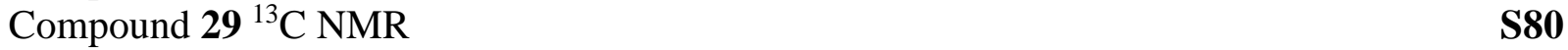

Figure S1. Automated Flash Chromatogram of $1 \quad$ S81

Figure S2. Automated Flash Chromatogram of $4 \quad \mathbf{S 8 2}$

Figure S3. Automated Flash Chromatogram of $5 \quad$ S83

Figure S4. Automated Flash Chromatogram of $6 \quad \mathbf{S 8 4}$

$\begin{array}{ll}\text { Figure S5. Automated Flash Chromatogram of } 7 & \text { S85 }\end{array}$

Figure S6. Automated Flash Chromatogram of $8 \quad \mathbf{S 8 6}$

Figure S7. Automated Flash Chromatogram of $9 \quad$ S87

Figure S8. Automated Flash Chromatogram of $2 \quad$ S88

Figure S9. Automated Flash Chromatogram of $10 \quad 589$

Figure S10. Automated Flash Chromatogram of $11 \quad \mathbf{S 9 0}$

Figure S11. Automated Flash Chromatogram of $12 \quad$ S91

Figure S12. Automated Flash Chromatogram of $13 \quad 592$

Figure S13. Automated Flash Chromatogram of $14 \quad$ S93

Figure S14. Automated Flash Chromatogram of $15 \quad 594$

Figure S15. Automated Flash Chromatogram of $16 \quad$ S95

Figure S16. Automated Flash Chromatogram of $17 \quad 596$

Figure S17. Automated Flash Chromatogram of $18 \quad$ S97

Figure S18. Automated Flash Chromatogram of $19 \quad 598$

Figure S19. Automated Flash Chromatogram of $20 \quad$ S99

Figure S20. Automated Flash Chromatogram of $21 \quad \mathbf{S 1 0 0}$

Figure S21. Automated Flash Chromatogram of 22 $\quad$ S101

Figure S22. Automated Flash Chromatogram of 23

Figure S23. Automated Flash Chromatogram of $24 \quad 5103$

Figure S24. Automated Flash Chromatogram of $25 \quad \mathbf{S 1 0 4}$

Figure S25. Automated Flash Chromatogram of $26 \quad \mathbf{S 1 0 5}$

Figure S26. Automated Flash Chromatogram of 27 $\quad$ S106

Figure S27. Automated Flash Chromatogram of $28 \quad$ S107

Figure S28. Automated Flash Chromatogram of $29 \quad \mathbf{S 1 0 8}$

Figure S29. LC/MS Chromatogram of $9 \quad$ S109

Figure S30. LC/MS Chromatogram of $2 \quad S 110$

Figure S31. LC/MS Chromatogram of $10 \quad 5111$

Figure S32. LC/MS Chromatogram of $11 \quad S 112$

Figure S33. LC/MS Chromatogram of $12 \quad \mathrm{S113}$

Figure S34. LC/MS Chromatogram of $13 \quad S 114$

Figure S35. LC/MS Chromatogram of $14 \quad \mathbf{S 1 1 5}$

Figure S36. LC/MS Chromatogram of $15 \quad S 116$

$\begin{array}{ll}\text { Figure S37. LC/MS Chromatogram of } 16 & \text { S117 }\end{array}$

Figure S38. LC/MS Chromatogram of $17 \quad$ S118

Figure S39. LC/MS Chromatogram of $18 \quad S 119$

Figure S40. LC/MS Chromatogram of $19 \quad 5120$ 
Table of Contents, cont.

Figure S41. LC/MS Chromatogram of $\mathbf{2 0}$

Figure S42. LC/MS Chromatogram of $\mathbf{2 1}$

S122

Figure S43. LC/MS Chromatogram of $\mathbf{2 2}$

S123

Figure S44. LC/MS Chromatogram of 23

S124

Figure S45. LC/MS Chromatogram of 24

S125

Figure S46. LC/MS Chromatogram of 25

S126

Figure S47. LC/MS Chromatogram of $\mathbf{2 6}$

S127

Figure S48. LC/MS Chromatogram of 27

S128

Figure S49. LC/MS Chromatogram of $\mathbf{2 8}$

S129

Tables S1-S7. X-ray crystallographic data for major regioisomer 2

S130

Tables S8-S14. X-ray crystallographic data for minor regioisomer 3

S143

Tables S15-S17. Computational data supporting information

S156 


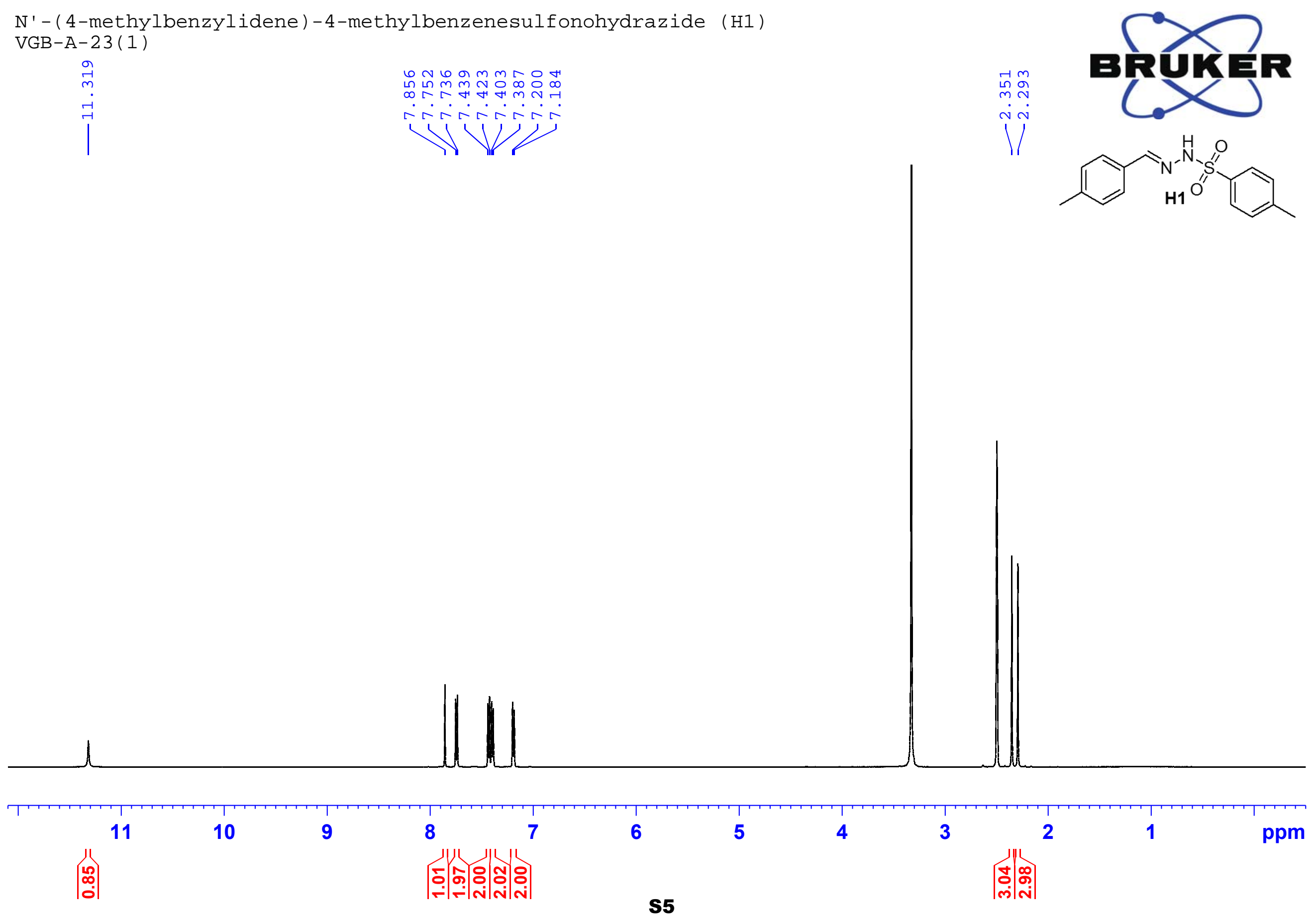


$N^{\prime}-(\mathrm{N}, \mathrm{N}$-dimethylaminobenzylidene)-4-methylbenzenesulfono-hydrazide (H2) VGB-B-43(5)
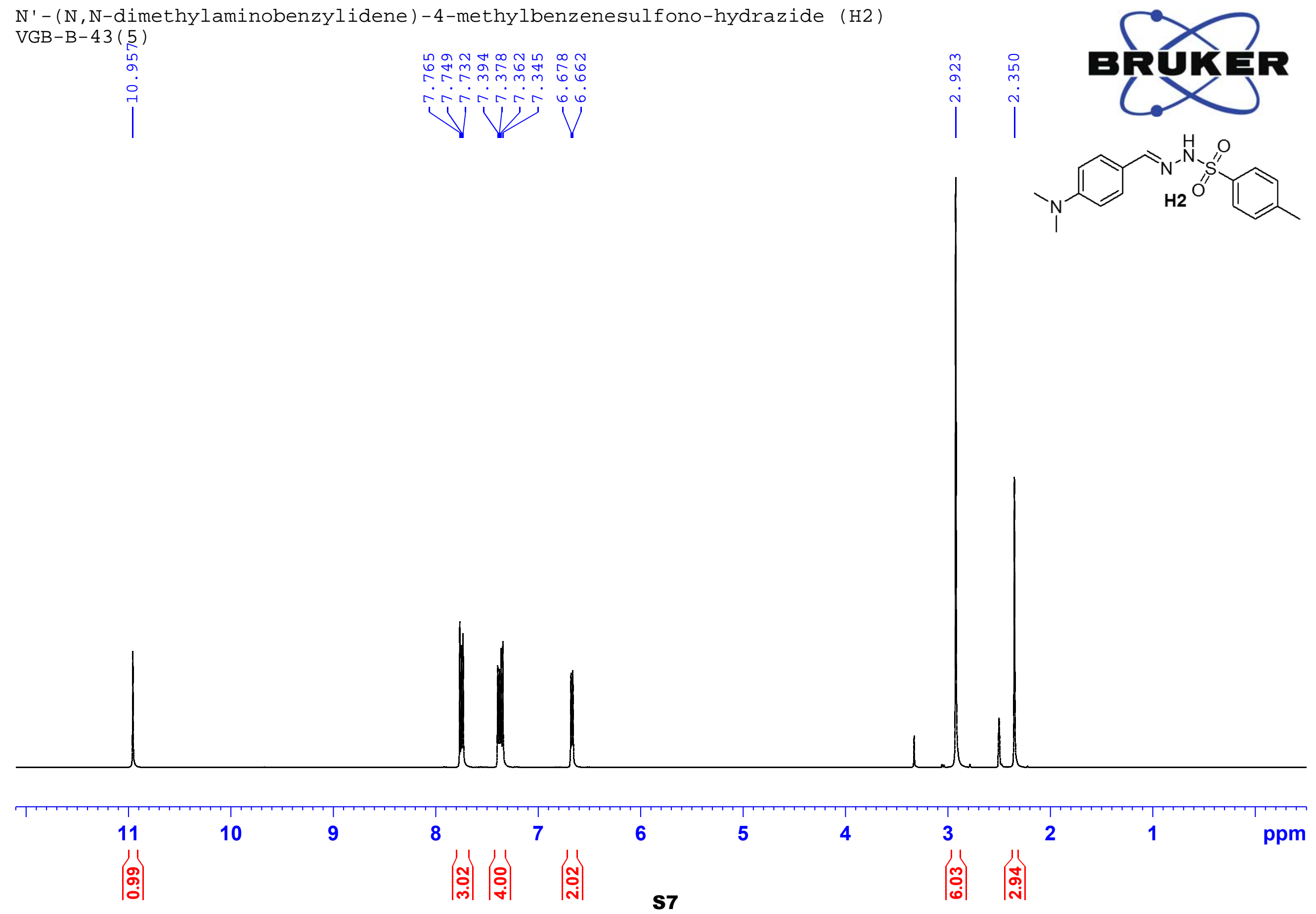


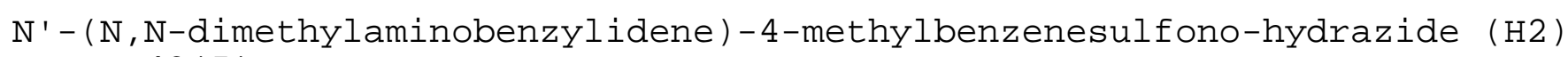
VGB-B-43(5)
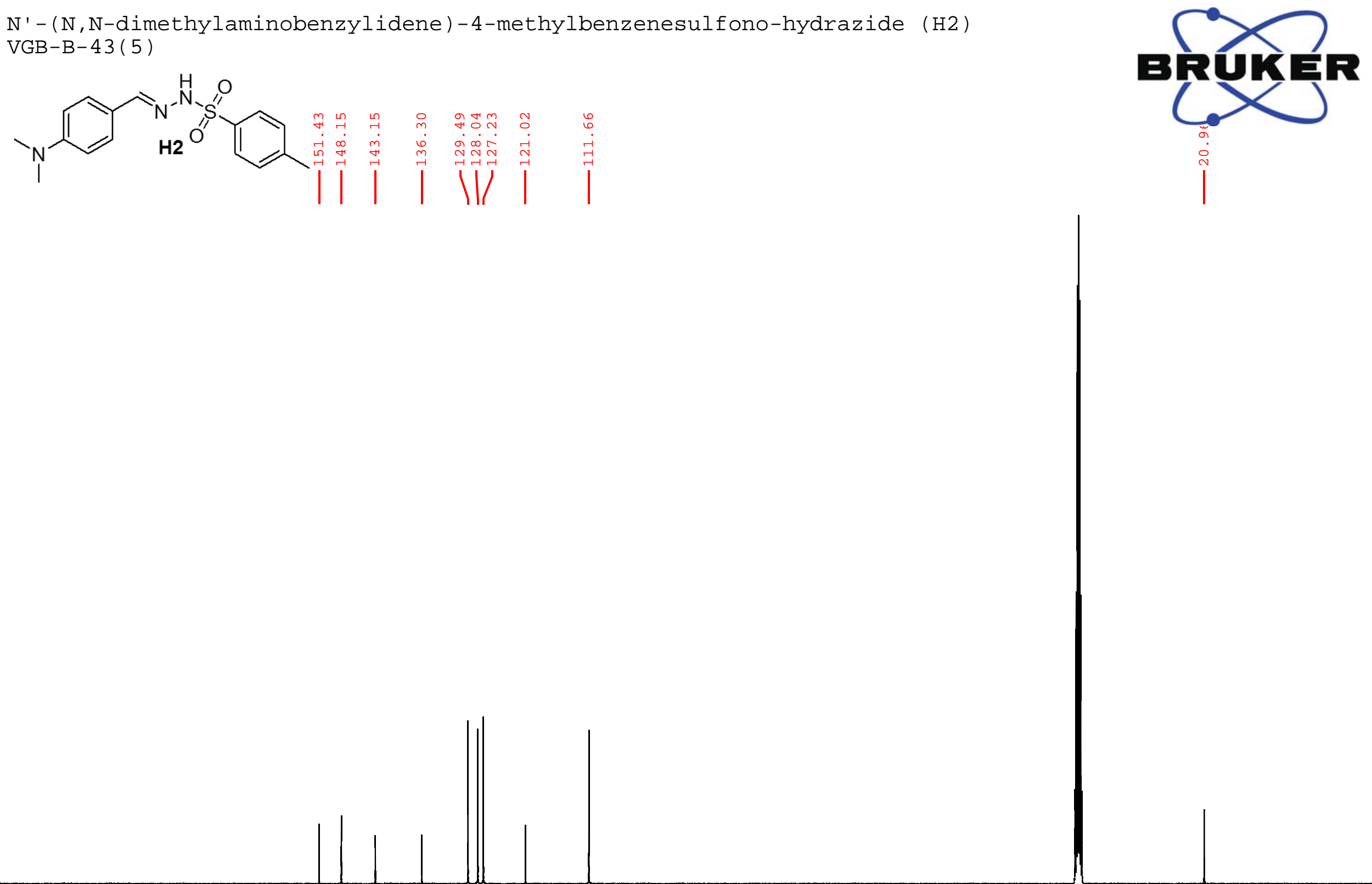

200

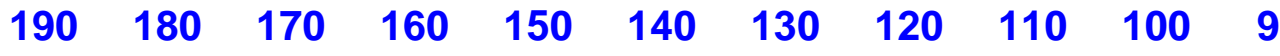

ग0 80

807060


$N^{\prime}$ - (2-iodobenzylidene) - 4-methylbenzenesulfonohydrazide (H3) VGB-B-197(1)
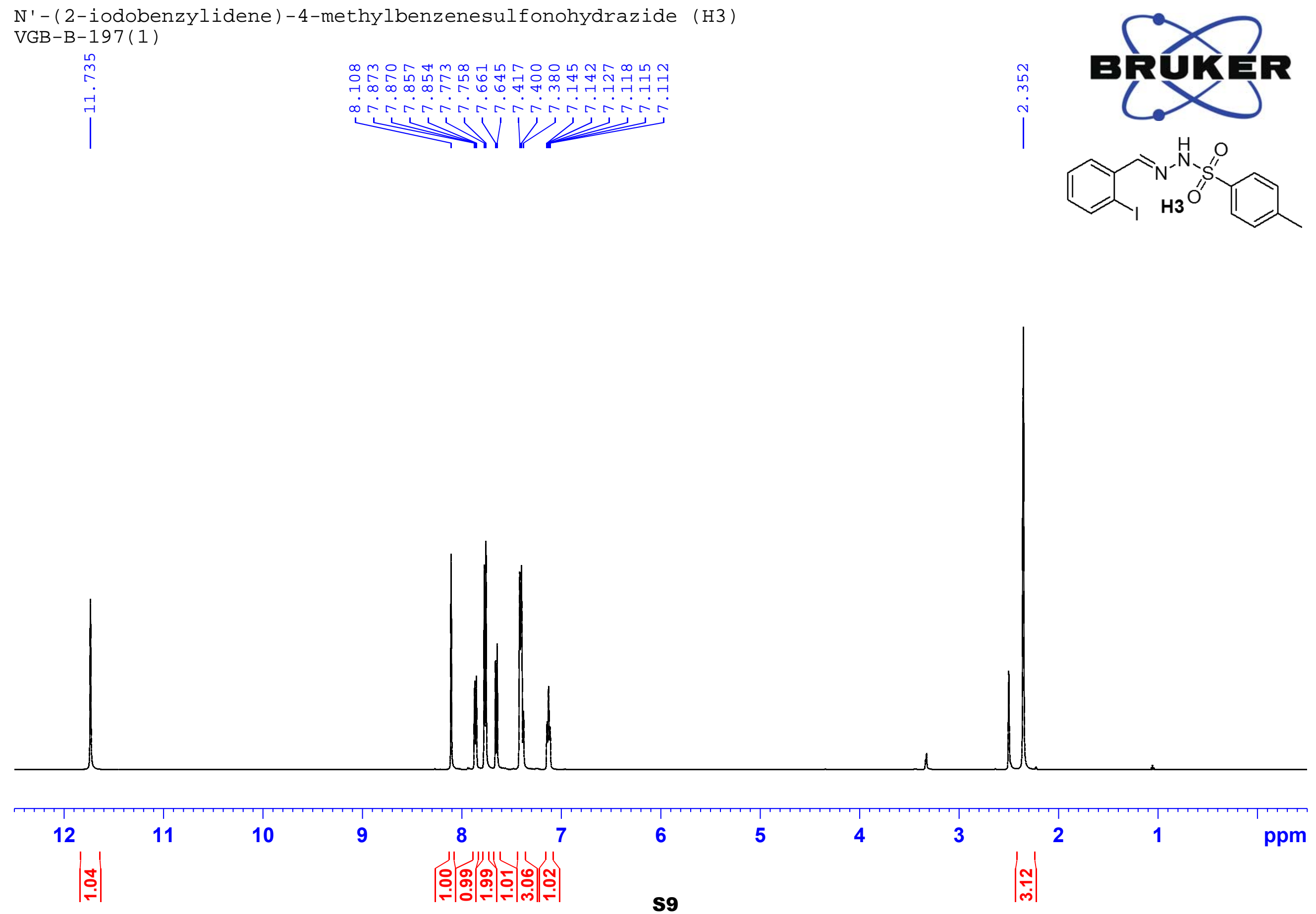
$N^{\prime}$ - (2-iodobenzylidene)-4-methylbenzenesulfonohydrazide (H3)

VGB-B-197(2)
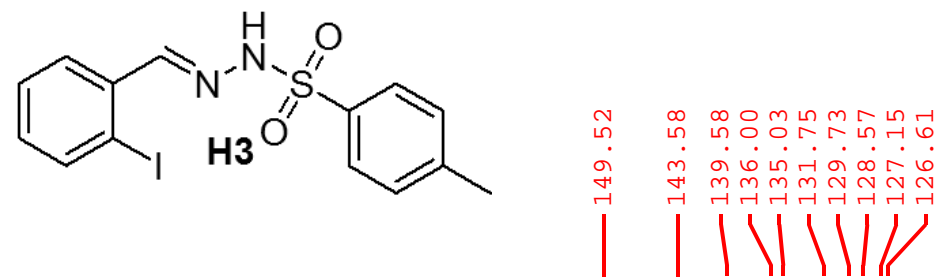

7
0
0
0

BefuKER (

$\stackrel{\mathfrak{N}}{1}$

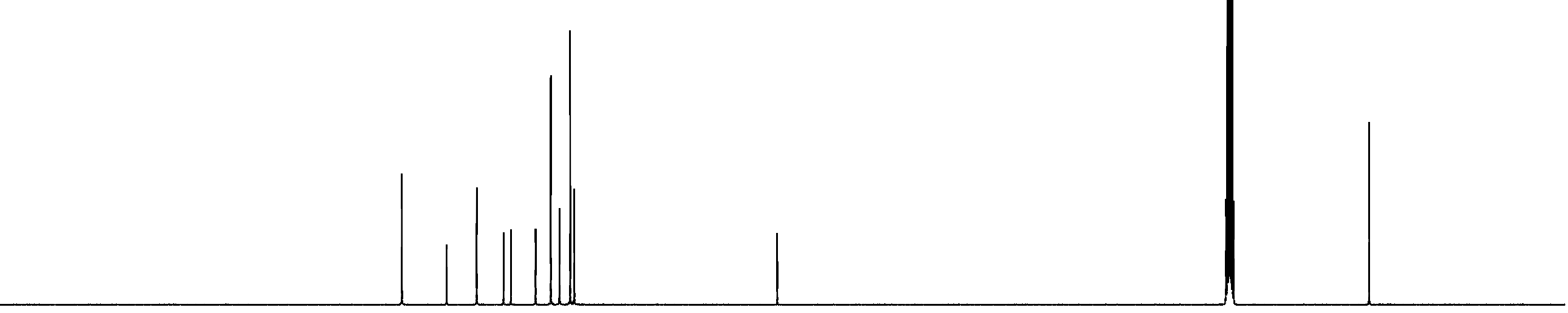

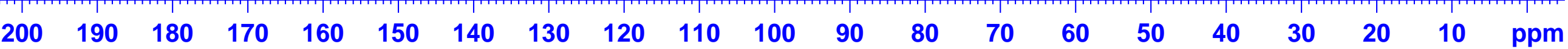


$N^{\prime}-(3,5-d i$-tert-butylbenzylidene)-4-methylbenzenesulfono-hydrazide (H4) VGB-B-169(1)
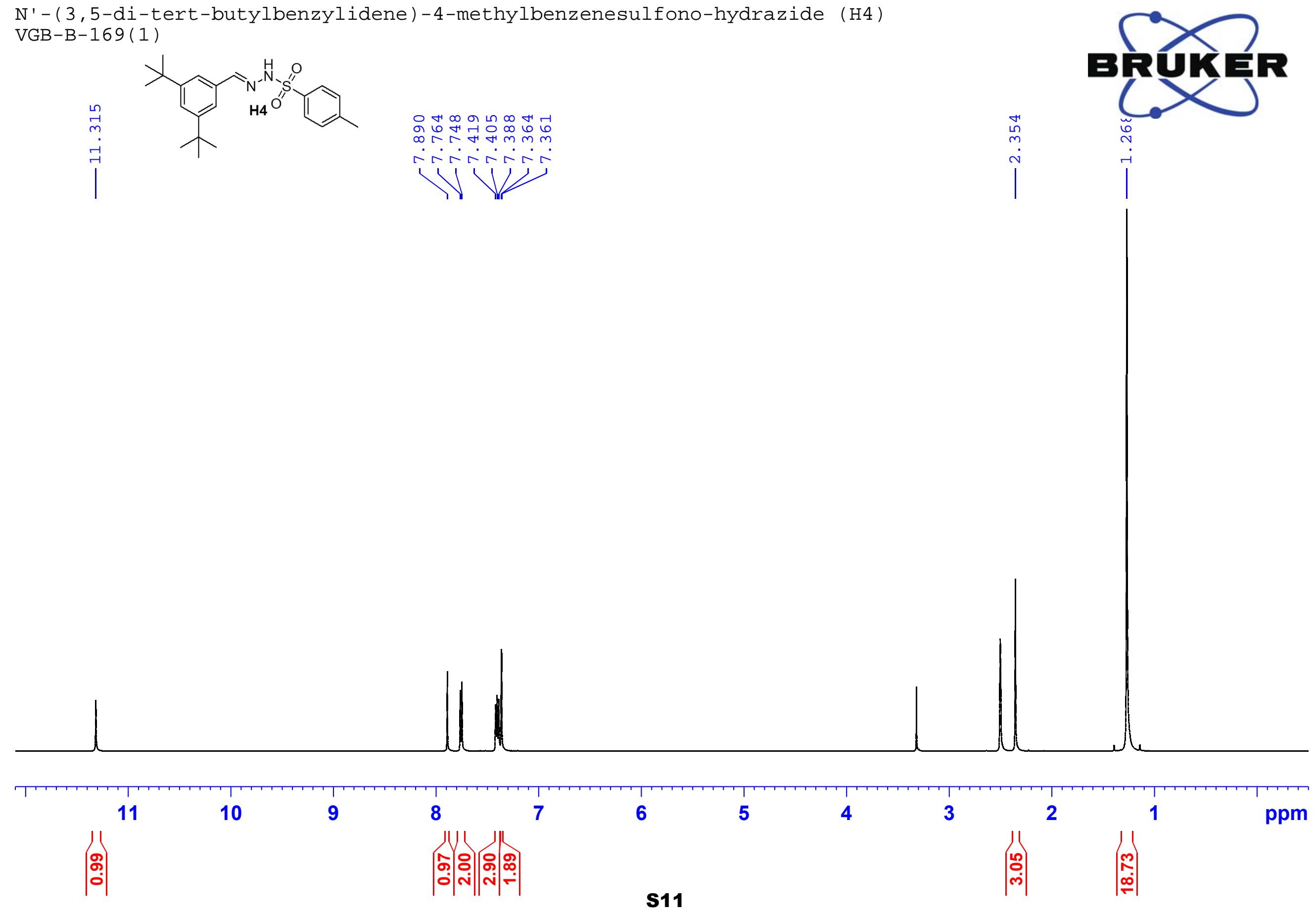
$N^{\prime}-(3,5-d i-t e r t-b u t y l b e n z y l i d e n e)-4-m e t h y l b e n z e n e s u l f o n o-h y d r a z i d e ~(H 4)$ VGB-B-169(5)
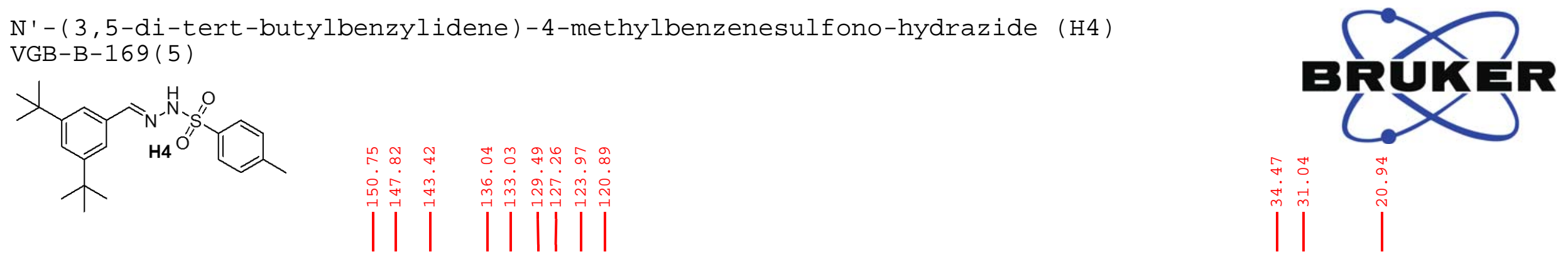

$\stackrel{\dot{\sim}}{\sim}$

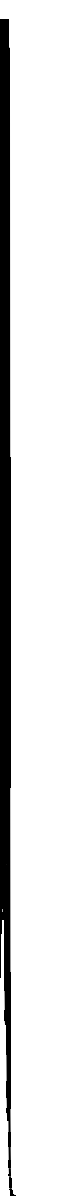

20

190180

180170

160

150

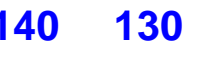

120

110

100

90

80

$70 \quad 60$

60

$50 \quad 40$ 
N'-(pyridinizylidene) - 4-methylbenzenesulfono-hydrazide (H5) VGB-B-115(1)
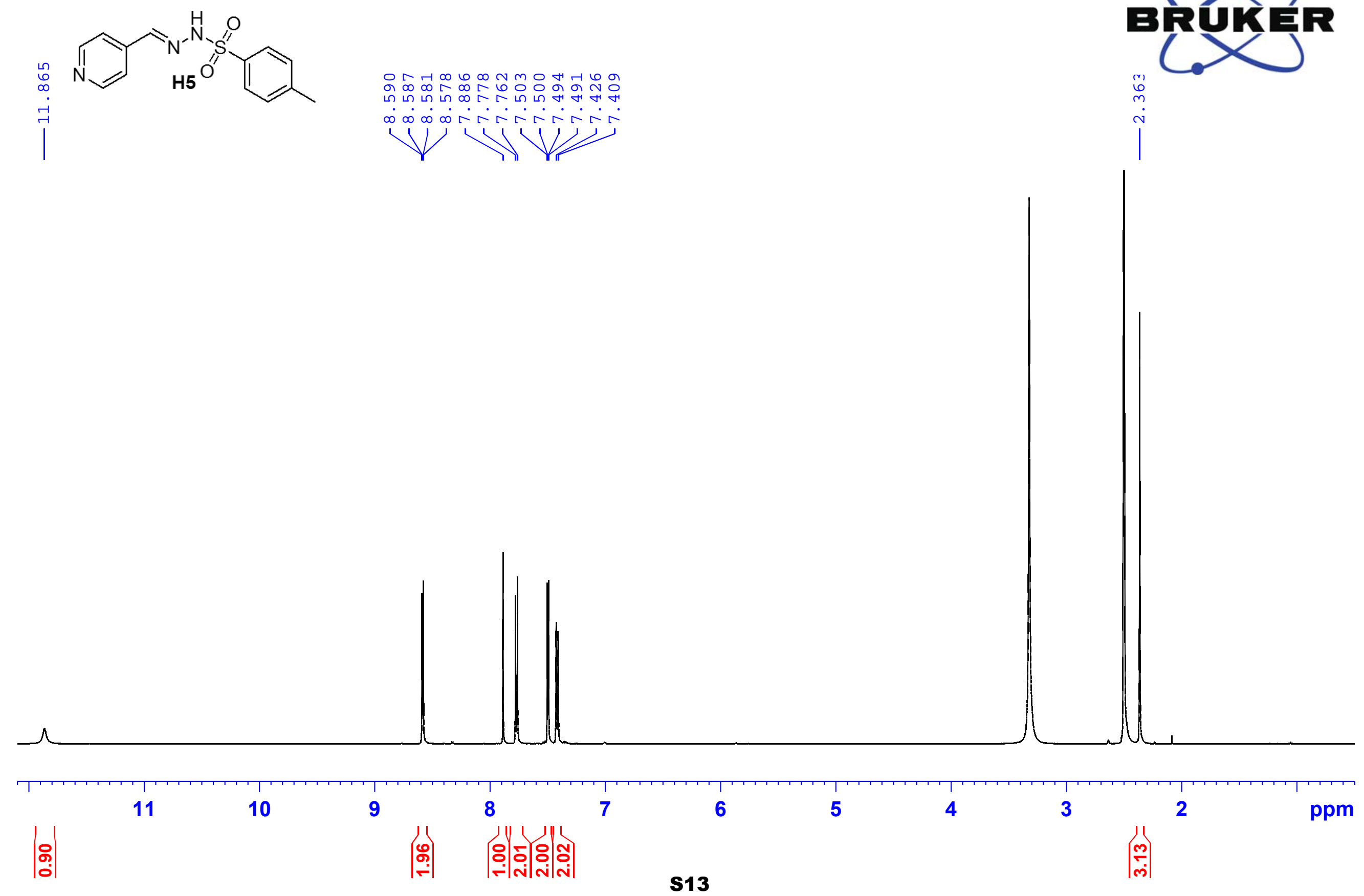
$N^{\prime}$ - (pyridin-4-yl-benzylidene)-4-methylbenzenesulfono-hydrazide (H5) VGB-B-115(4)
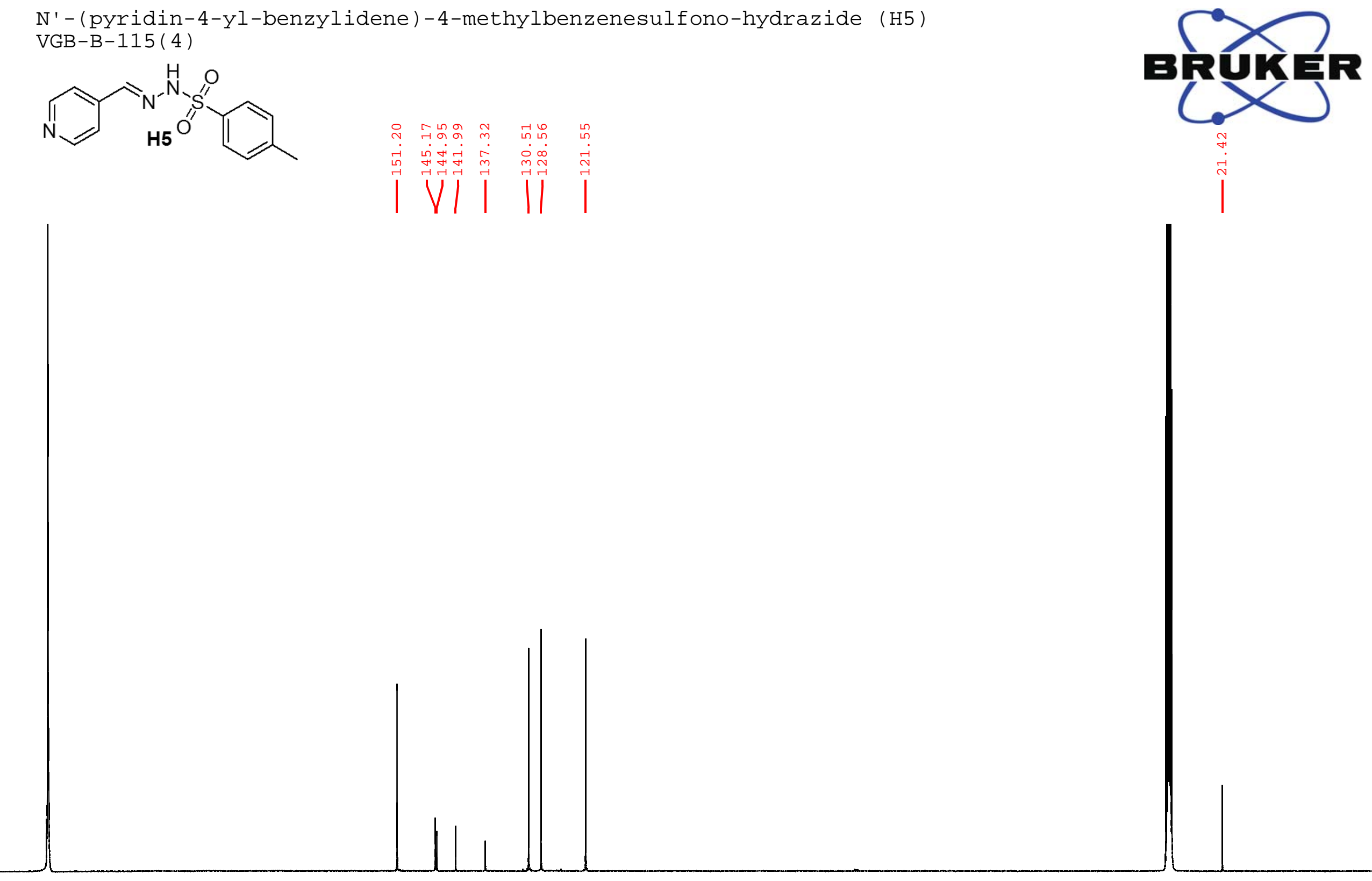

\begin{tabular}{|c|c|c|c|c|c|c|c|c|c|c|c|c|c|c|c|c|c|c|}
\hline 200 & 190 & 180 & 170 & 160 & 150 & 140 & 130 & 120 & 110 & 100 & 90 & 80 & 70 & 60 & 50 & 40 & 30 & 10 \\
\hline
\end{tabular}


$N^{\prime}$ - (4-tert-butylbenzylidene)-4-methylbenzenesulfonohydrazide (H6) VGB-B-69(5)

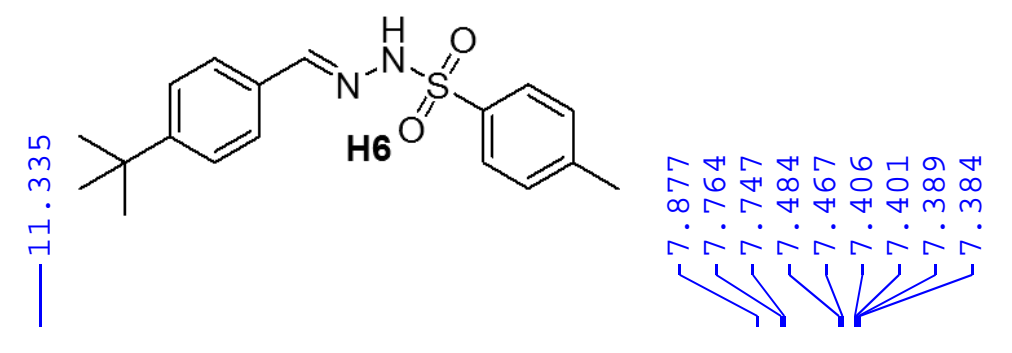

gêu

离

$(->)$

$\stackrel{\overbrace{}}{\sim}$

í

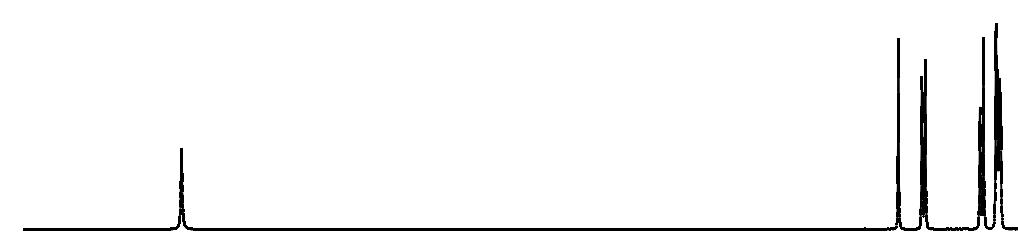

$U$

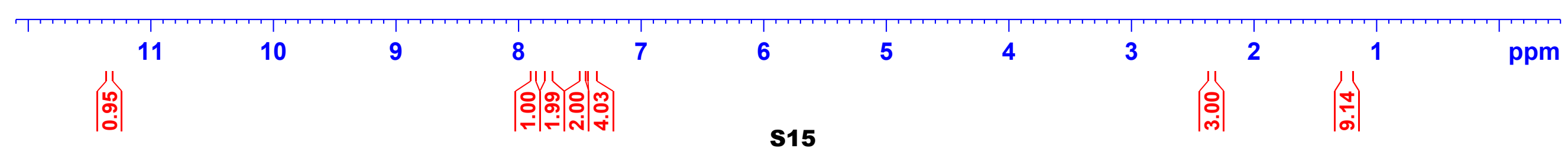




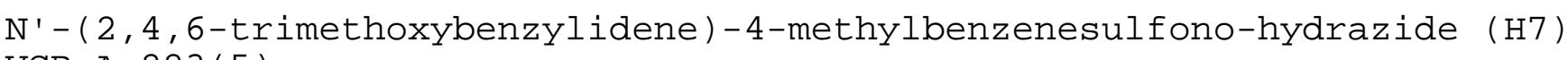
VGB-A-223(5)
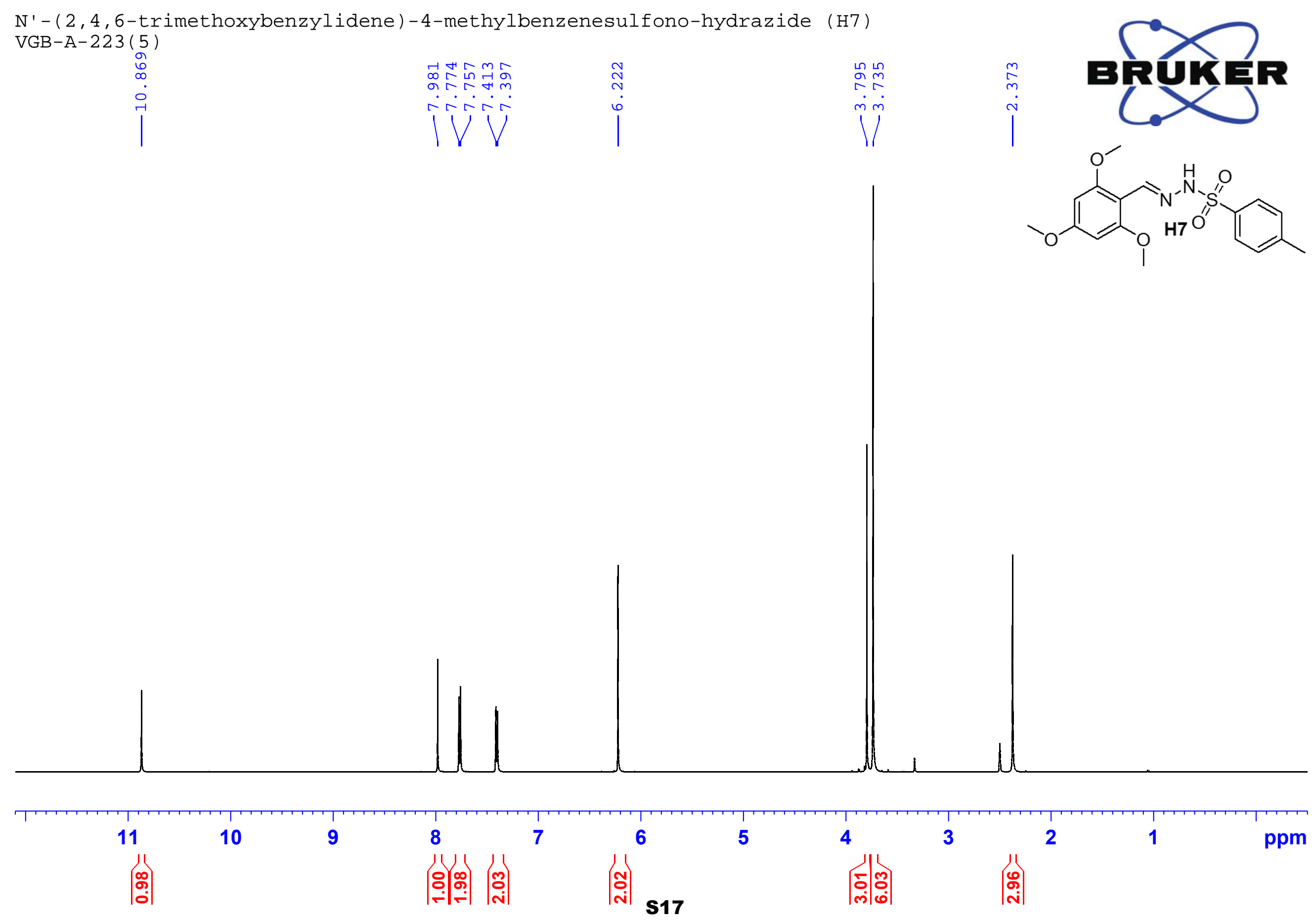
$N^{\prime}-(2,4,6$-trimethoxybenzylidene $)$-4-methylbenzenesulfono-hydrazide (H7) VGB-A-223(5)
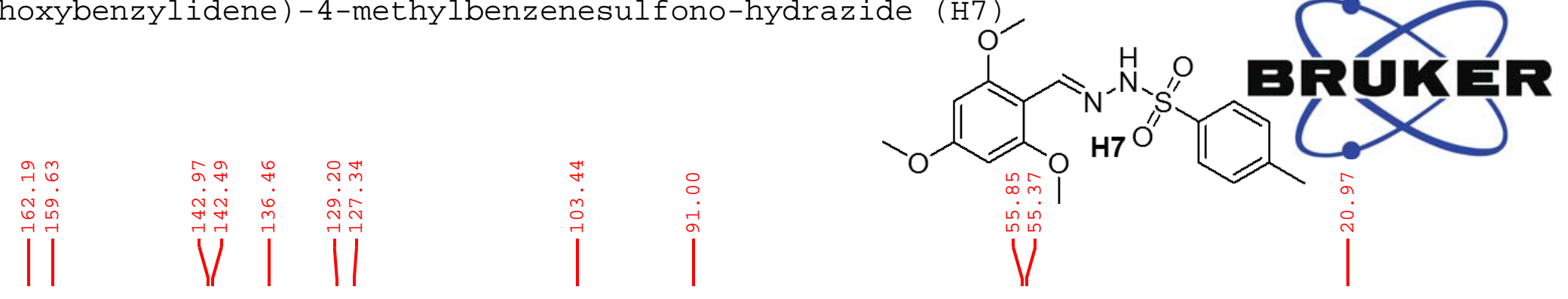

200

190

180

$170 \quad 160$

150

140

130120

110

100

90

80

70

60

50

40 
$N^{\prime}-($ C-6-Bromo-pyridin-2-yl)-4-methylbenzenesulfono-hydrazide (H8) VGB-B-41(1)

ํํํ

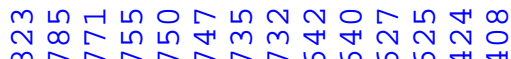

icisiciciticis

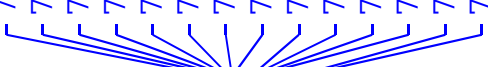

ङे

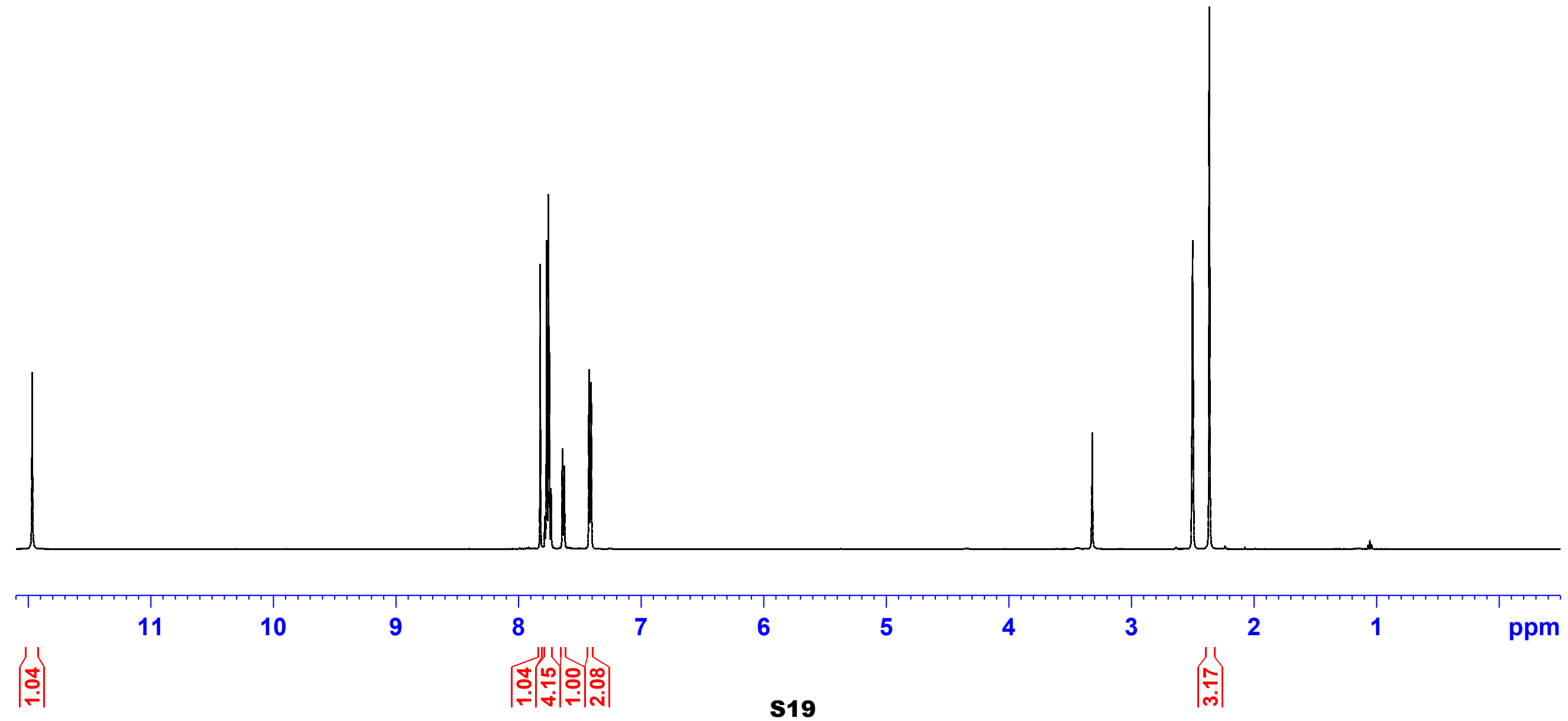


$N^{\prime}-($ C-6-Bromo-pyridin-2-yl)-4-methylbenzenesulfono-hydrazide (H8) VGB-B-41(1)
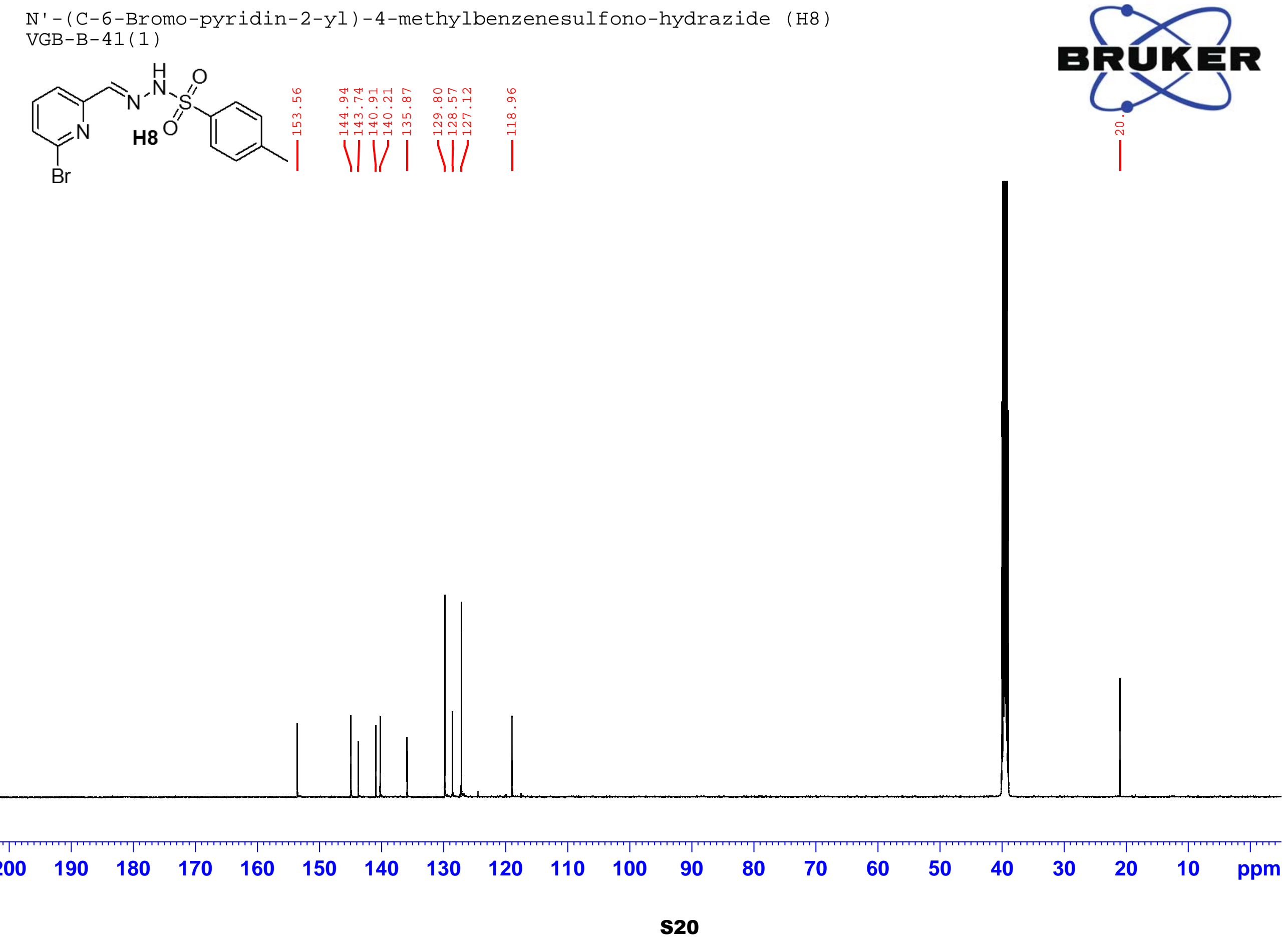
N'-(2-bromo-4-tert-butyl-6-phenol benzylidene)-4-methylbenzenesulfono-hydrazide (H9)

VGB-B-237(1)

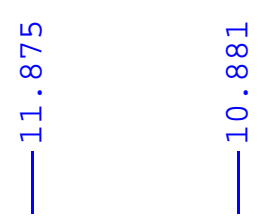

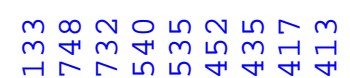
oninininina 11
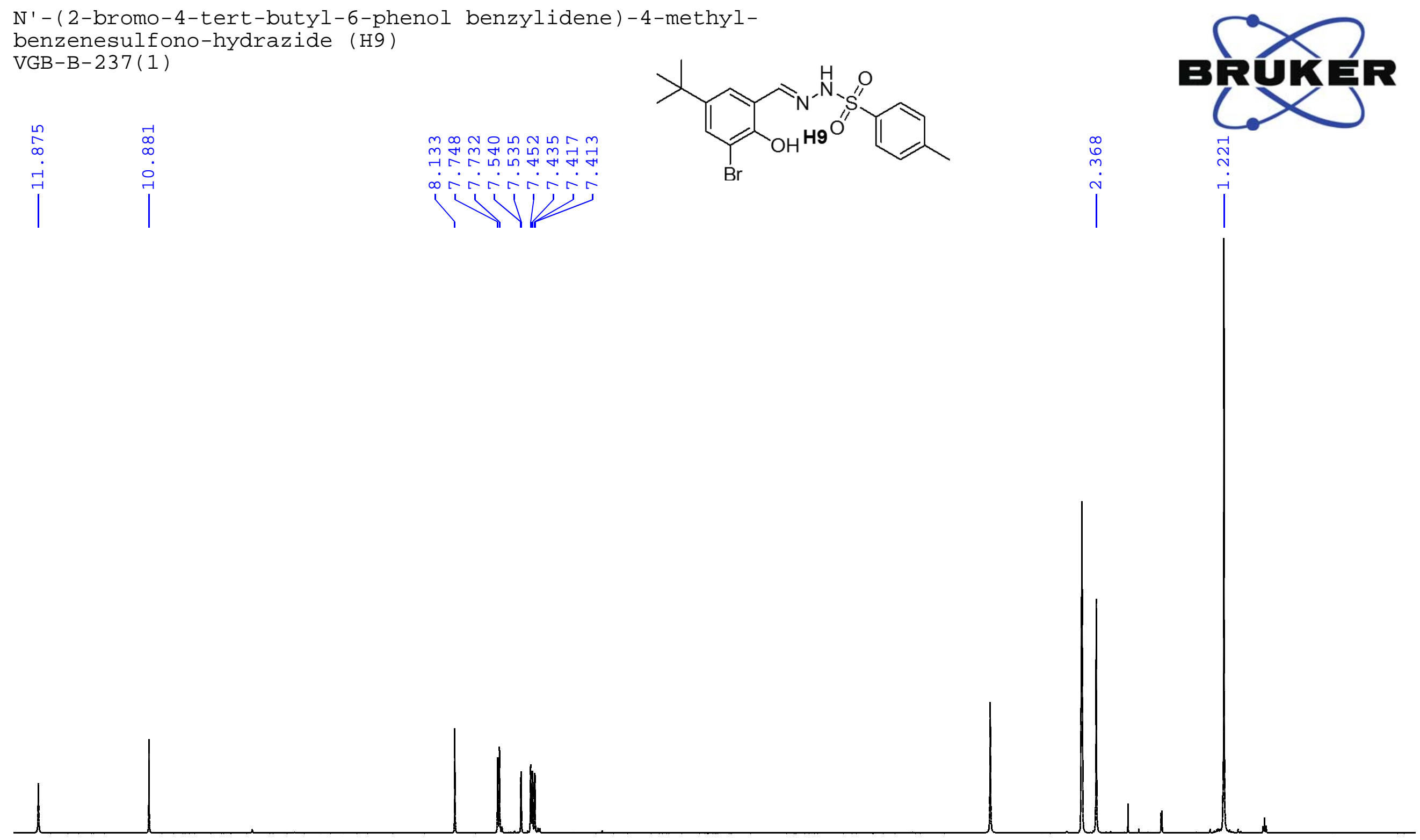

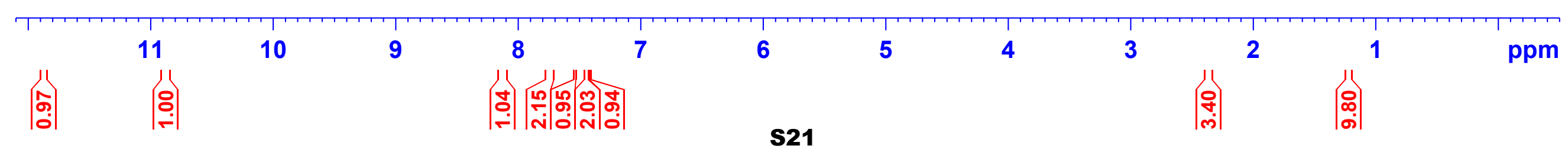


$\mathrm{N}^{\prime}$ - (2-bromo-4-tert-butyl-6-phenol benzylidene)-4-methylbenzenesulfono-hydrazide (H9)

VGB-B-237 (1)
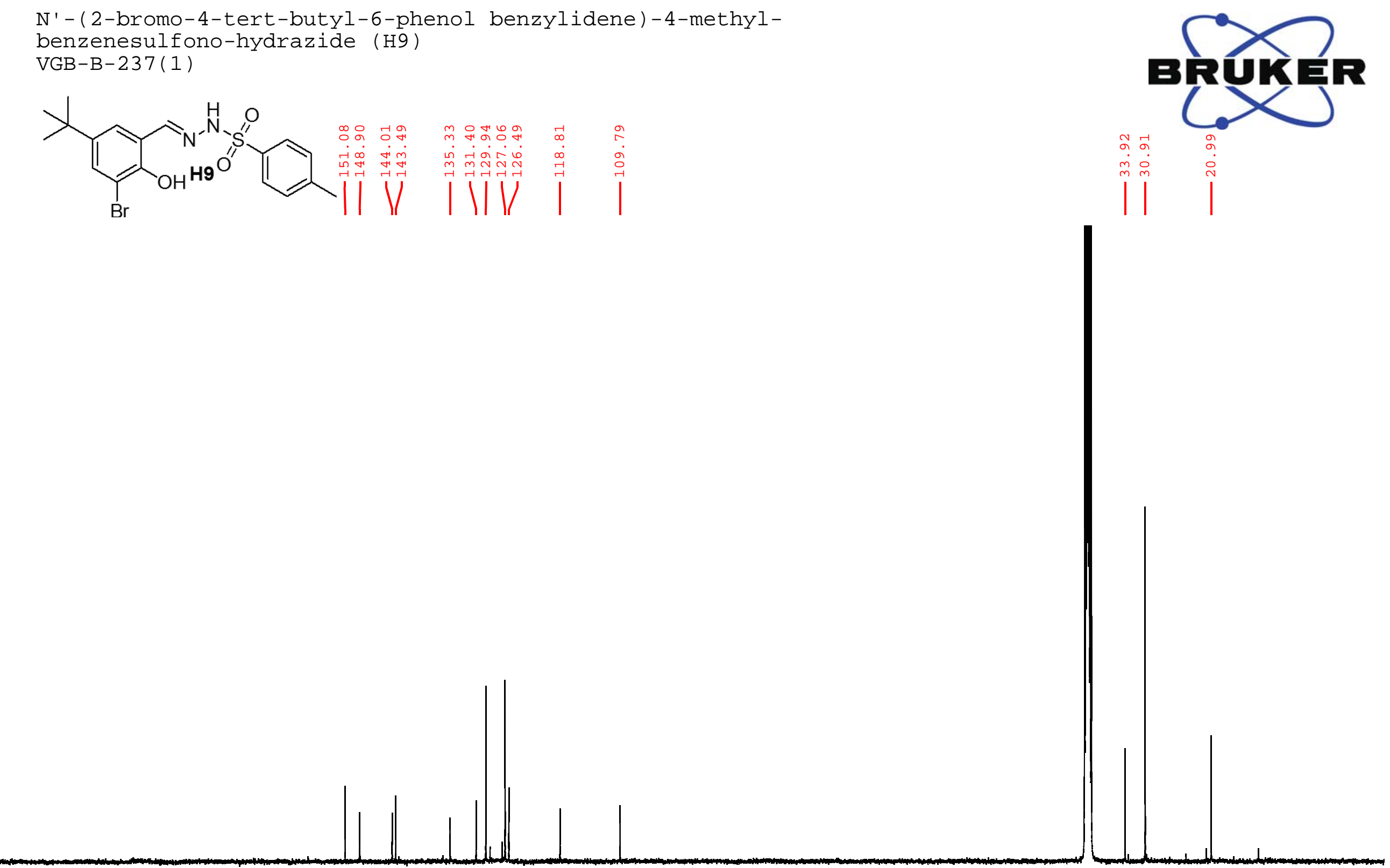

$\begin{array}{lllllllllllllllllllll}200 & 190 & 180 & 170 & 160 & 150 & 140 & 130 & 120 & 110 & 100 & 90 & 80 & 70 & 60 & 50 & 40 & 30 & 20 & 10 & \text { ppm }\end{array}$


3-(6-Ethynyl-pyridin-2-yl)-5,6-diphenyl-[1,2,4]triazine (1) VGB-B-177(1)

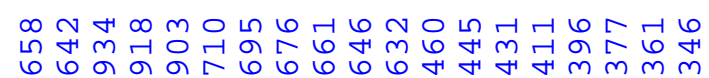

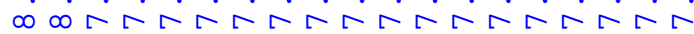

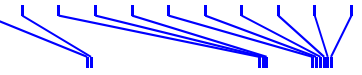
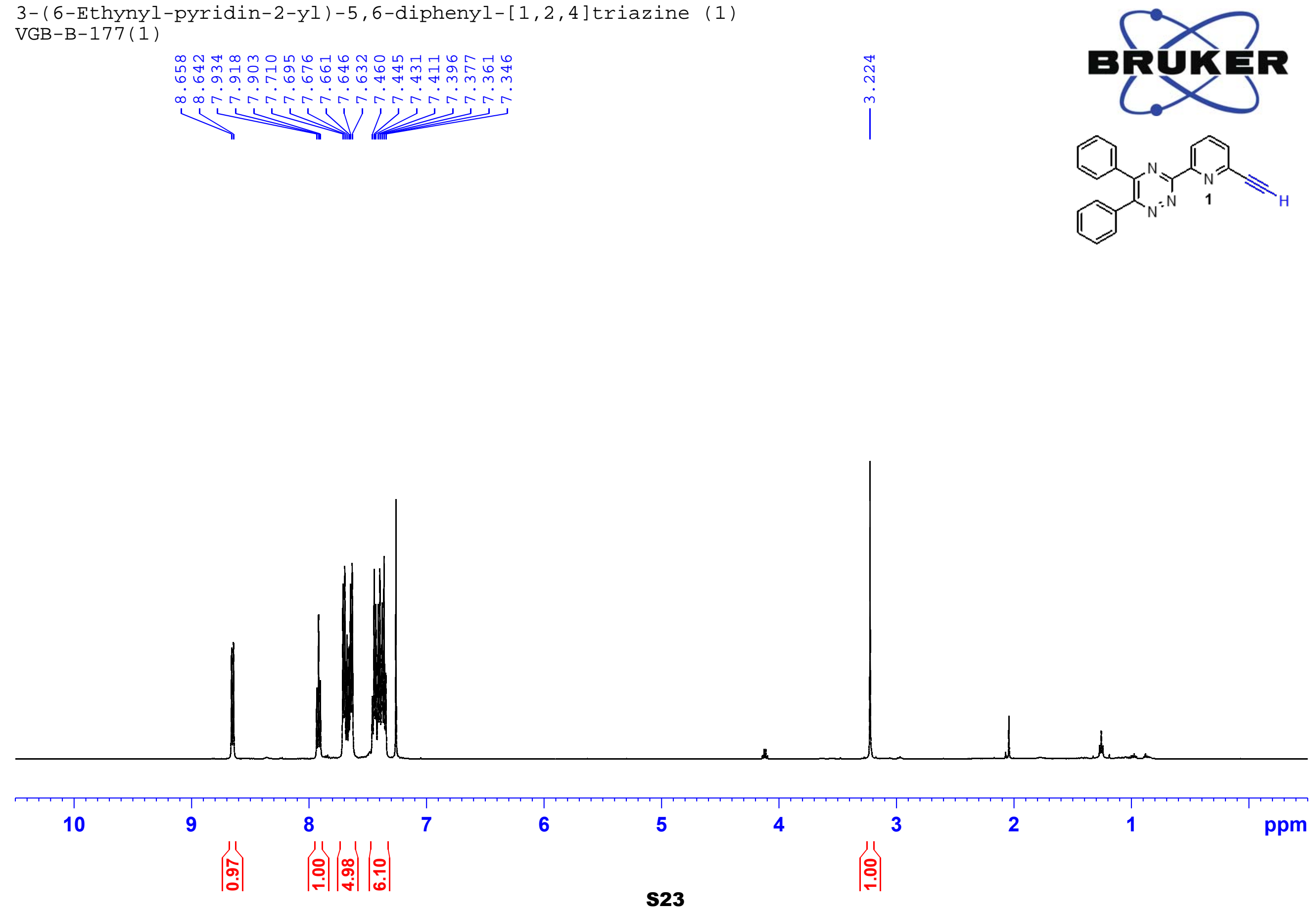
3-(6-Ethynyl-pyridin-2-yl)-5,6-diphenyl- $[1,2,4]$ triazine VGB-B-177(1)

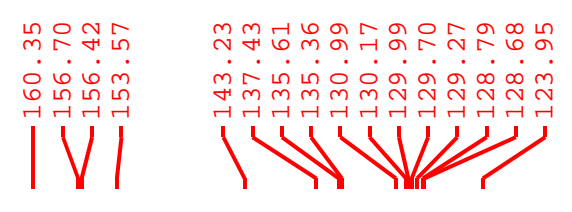

200
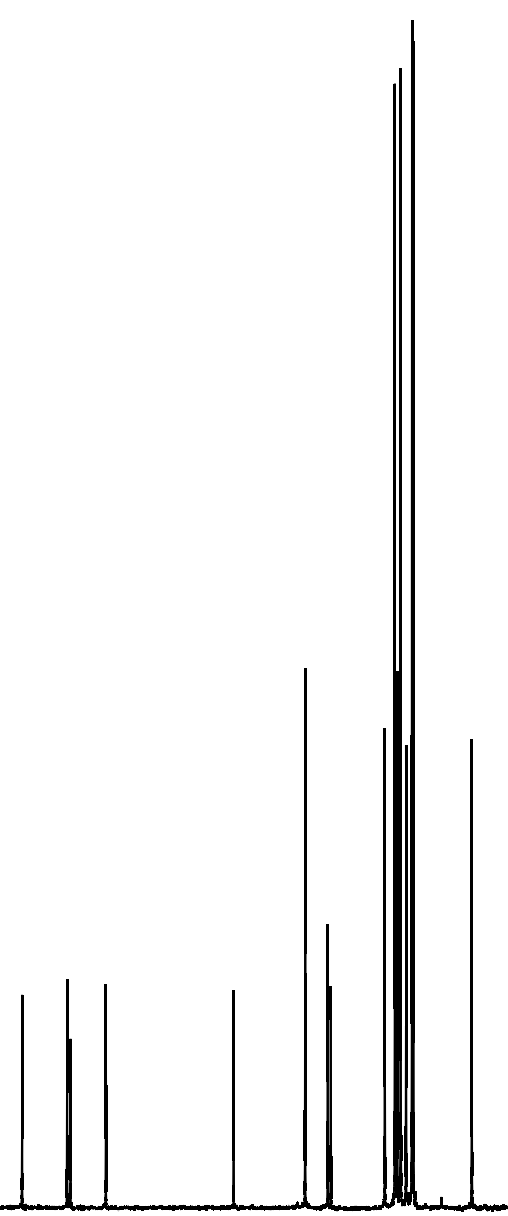

$\stackrel{n}{n}$

$\stackrel{\infty}{\infty} \stackrel{\infty}{\infty}$

$\left.\right|^{\infty}$

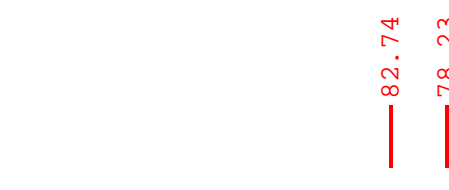

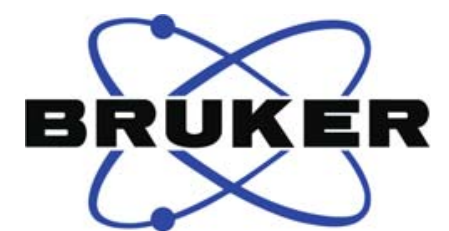

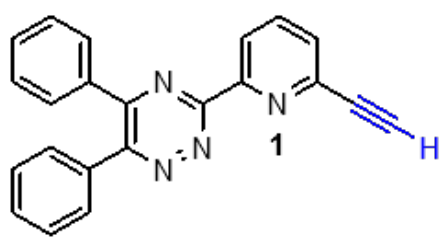


3-(6-Ethynyl-pyridin-2-yl)-5,6-di-p-tolyl-[1,2,4]triazine (4) VGB-B-103(5)

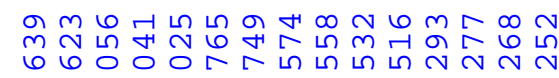

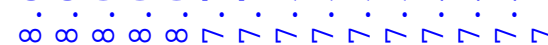

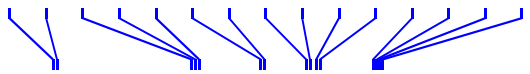

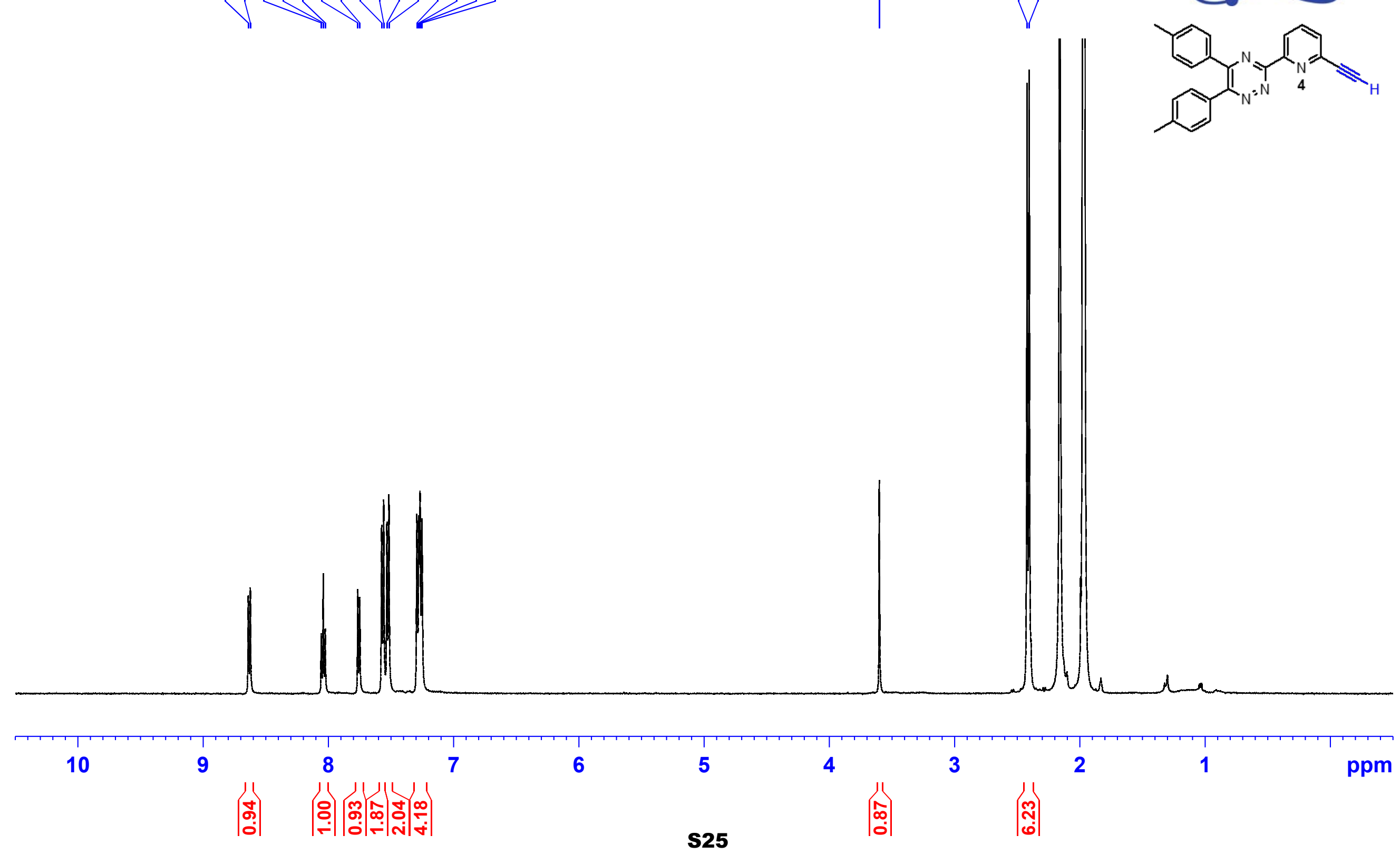


3-(6-Ethynyl-pyridin-2-yl) -5, 6-di-p-tolyl-[1,2,4]triazine (4) VGB-B-161(1)

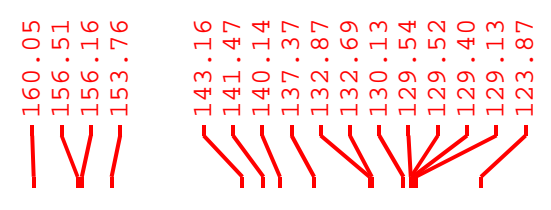

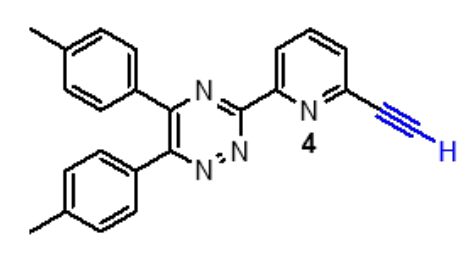

$\begin{array}{ll}-1 & 7 \\ \infty & 7 \\ \infty & \infty \\ \infty & 1 \\ \mid & 1\end{array}$
BRUIA

$\stackrel{\text { กิ }}{\text { ก }}$

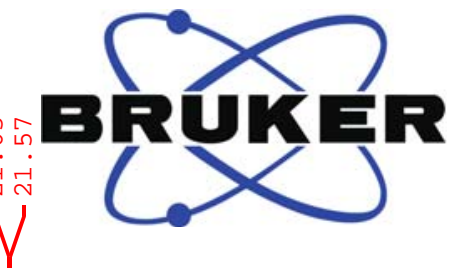

20

190180

170

160

150140

130

120

$110 \quad 100$

90

80

$70 \quad 60$

50

40

30

20

10

ppm 
5,6-Bis-(4-butyl-phenyl)-3-(6-ethynyl-pyridin-2-yl)-[1,2,4] triazine (5) VGB-B-161(1)

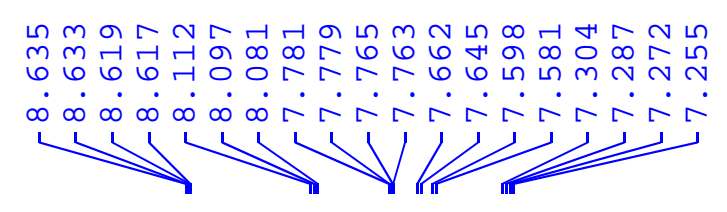

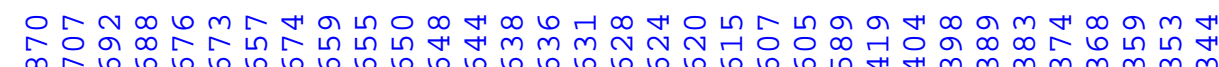

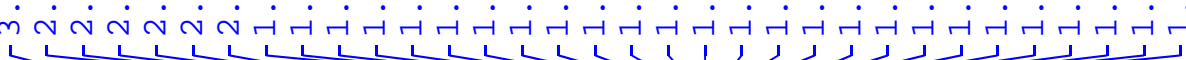

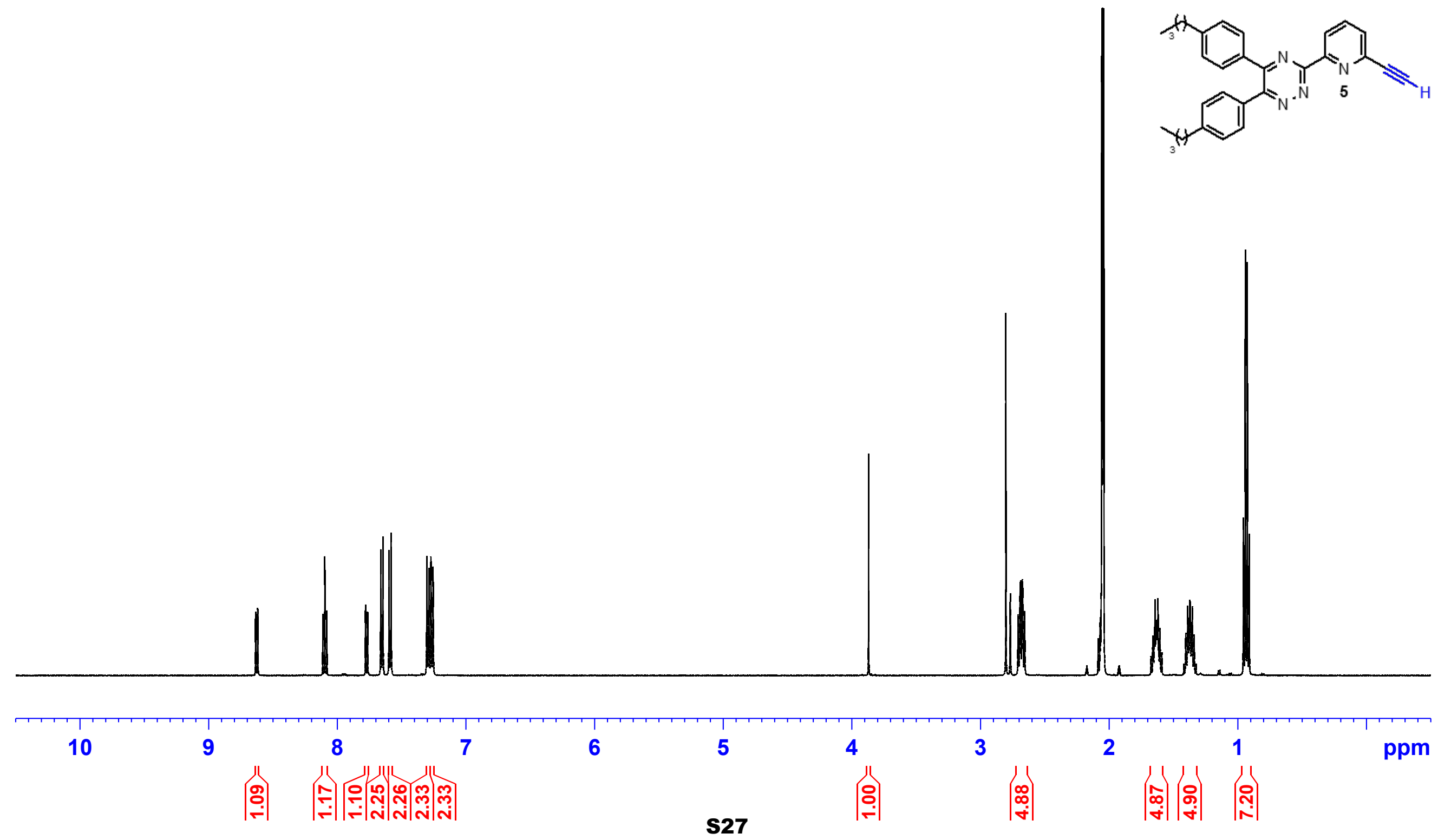


3 - (6-Ethynyl-pyridin-2-yl) -5,6-bis- (4-fluoro-phenyl) $[1,2,4]$ triazine (6)

VGB-B-83(1)

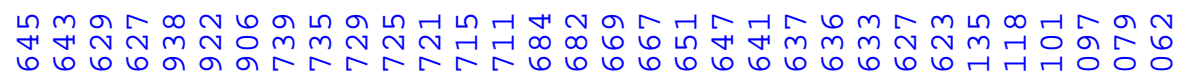

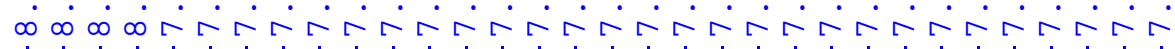
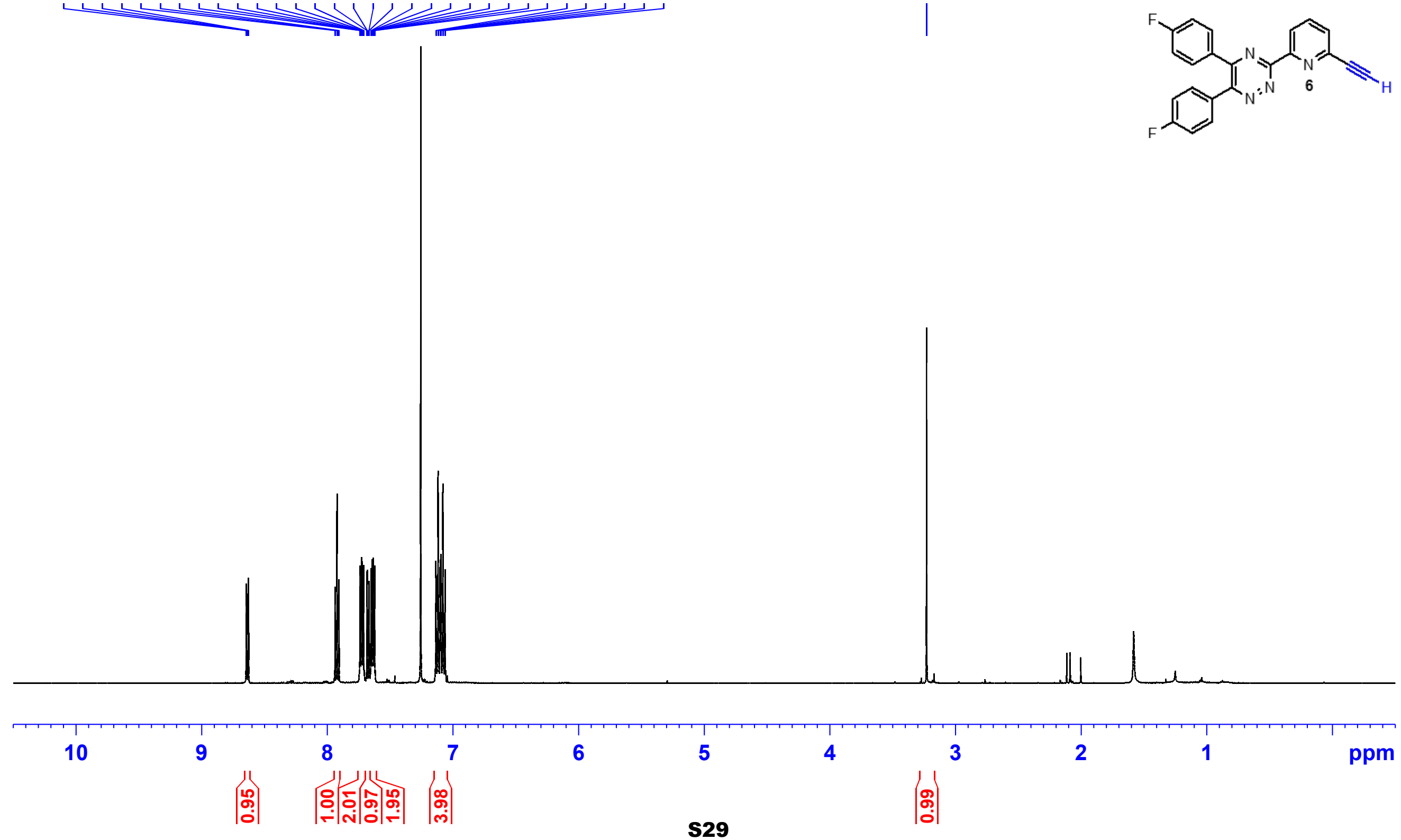
3-(6-Ethynyl-pyridin-2-yl)-5,6-bis-(4-fluoro-phenyl)-[1,2,4] triazine (6) VGB-B-83(1)

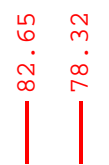

เึ. ले

$\stackrel{\infty}{\infty}$

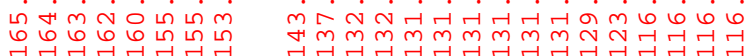
VI/VI
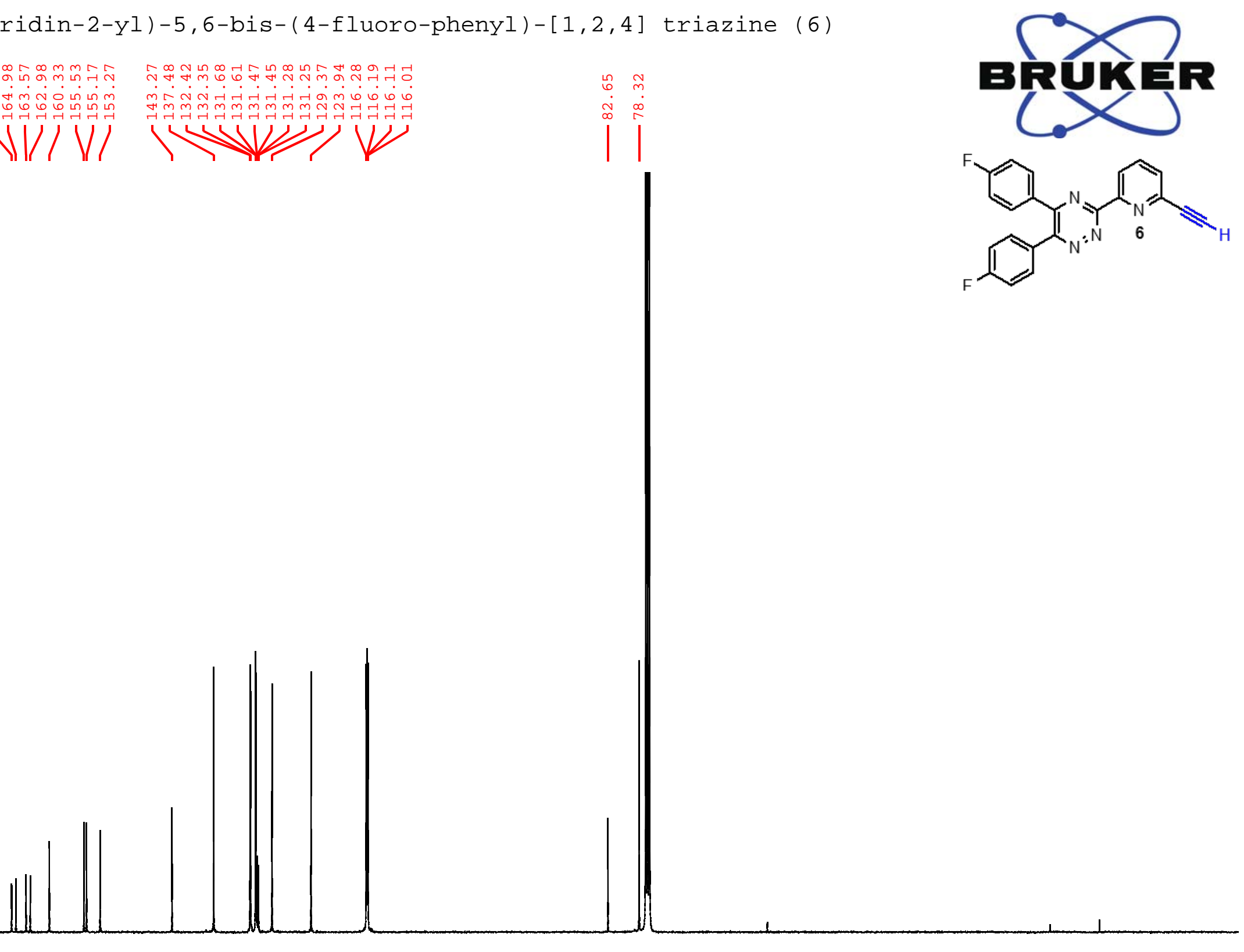

20

$\begin{array}{lll}190 \quad 180 & 170\end{array}$

160

150140

130

120

110

100

90

80

70

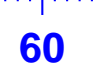

50

40

30

20

10 ppm 
5,6-Bis - [4-(3,3-dimethyl-butyl) - phenyl] - 3 - (6-ethynyl -

pyridin-2-yl)-[1,2,4]triazine (7)

VGB-B-91(2)

œ

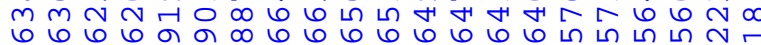

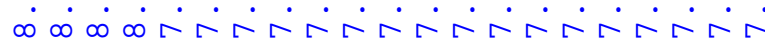
$\longrightarrow$

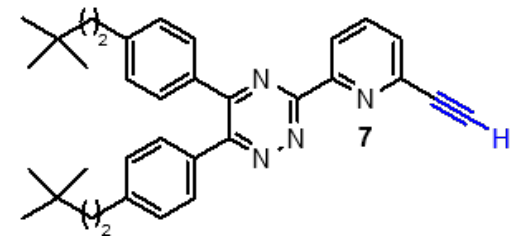

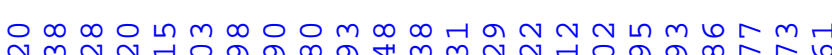
N

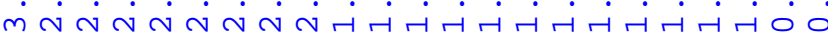

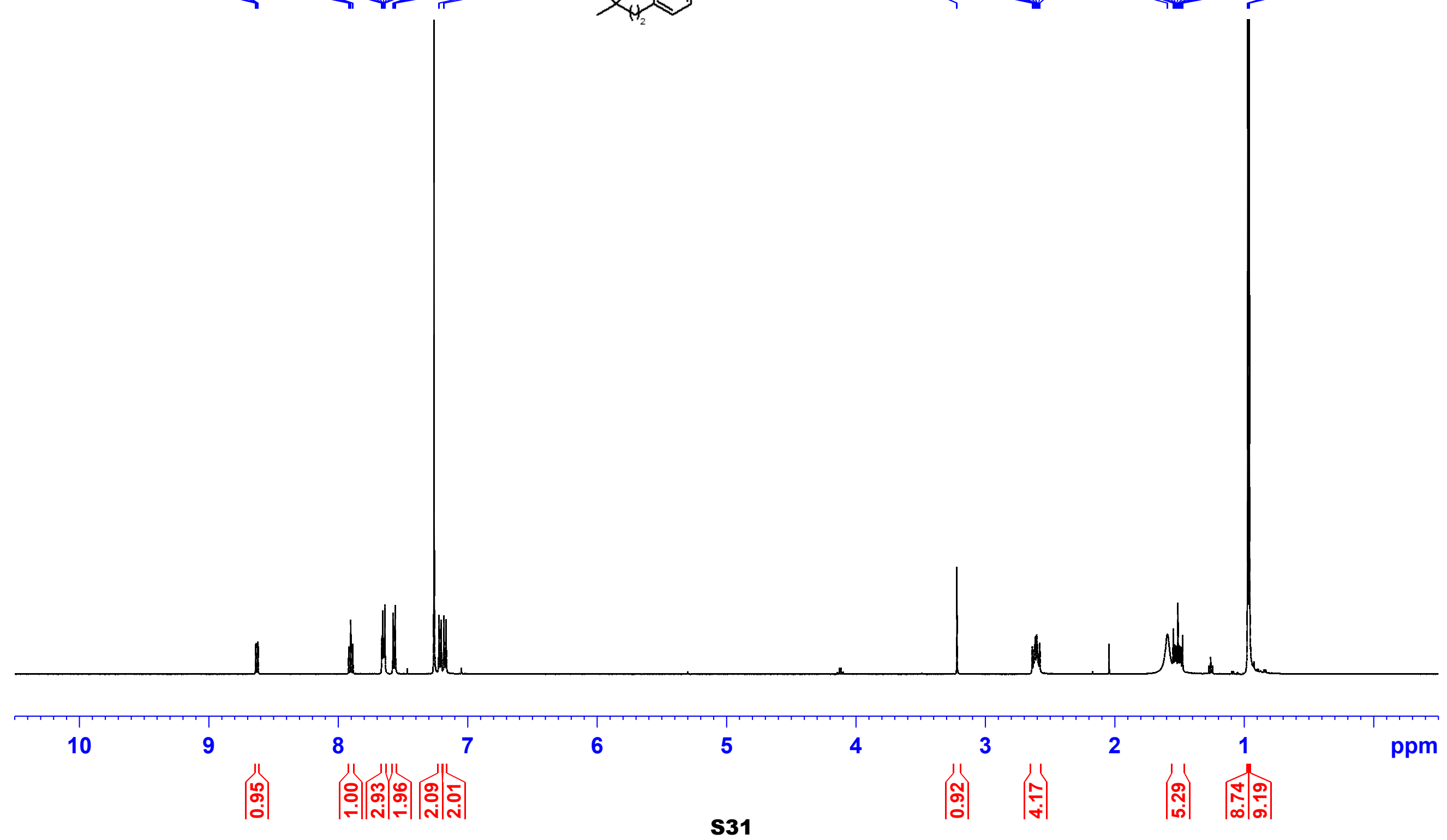


5,6-Bis-[4-(3,3-dimethyl-butyl)-phenyl]-3(6-ethynyl-pyridin-2-yl)-[1,2,4]triazine (7) VGB-B-91(1)

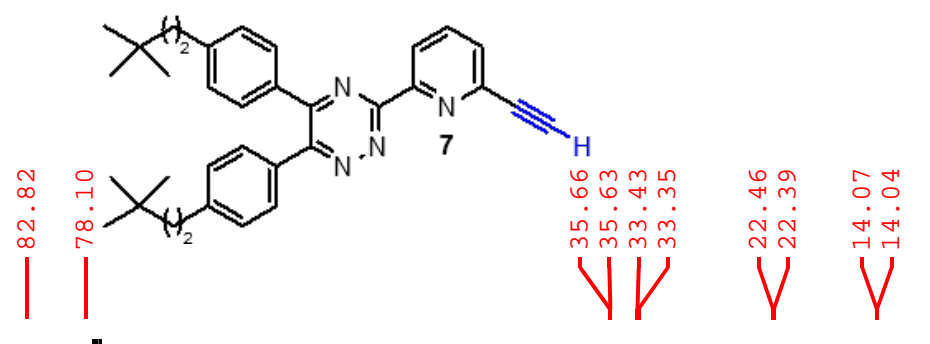


5,6-Bis-(4-cyclopropyl-phenyl) -3- (6-ethynyl-pyridin-2-yl)$[1,2,4]$ triazine $(8)$

VGB-B-263(2)

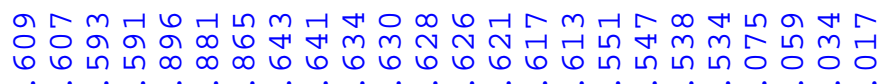

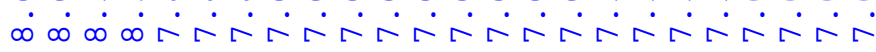

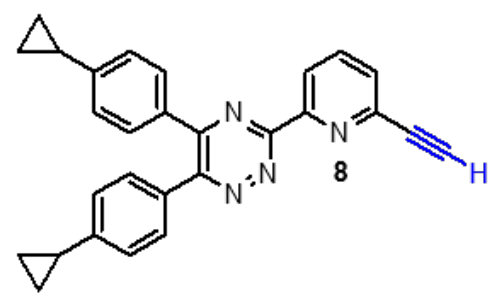

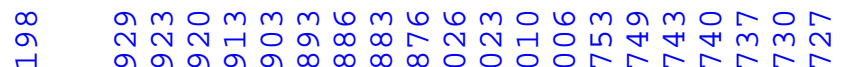

m
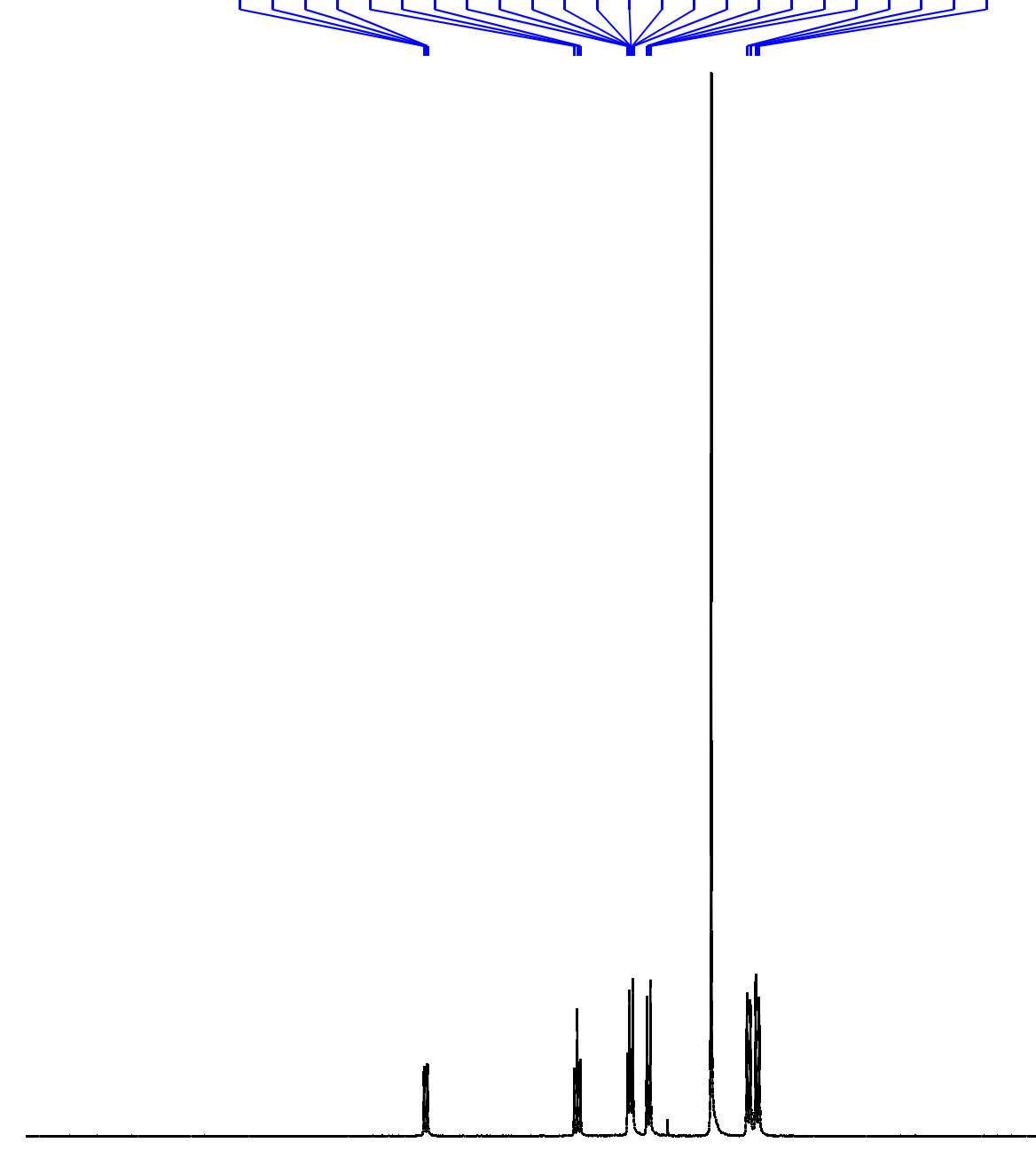

10

9

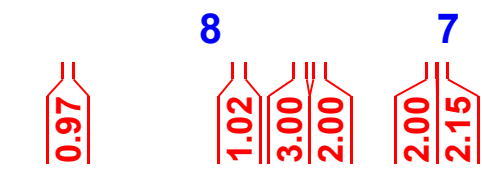

6

5

S33
4

3

$\left(\begin{array}{c}1 \\ \infty \\ 0 \\ 0\end{array} \mid\right.$
2

$\left|\frac{1}{i}\right|$ ppm 
5,6-Bis-(4-cyclopropyl-phenyl)-3-(6-ethynyl-pyridin-2-yl)- [1,2,4]triazine (8) VGB-B-263(1)

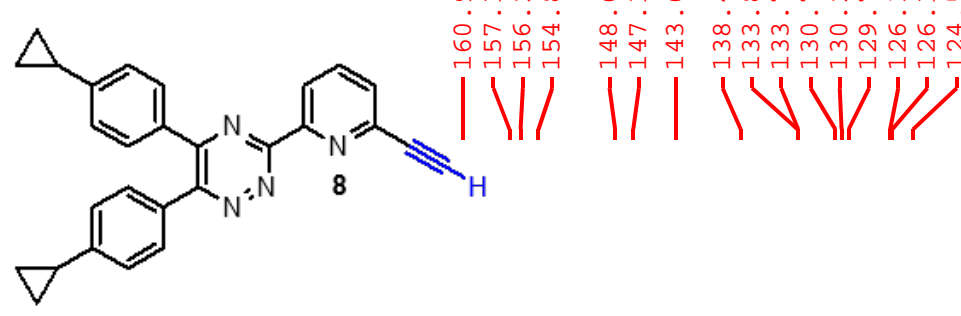

구

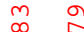

1

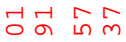

욱용ㅇㅇ

V V

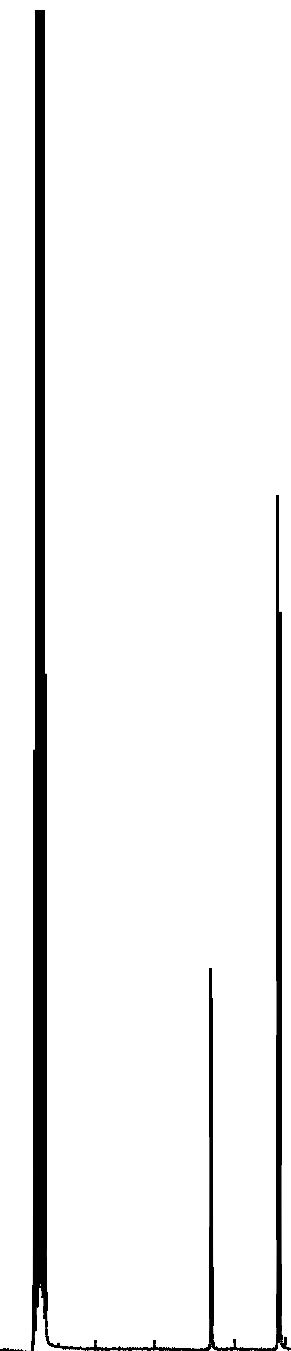

20

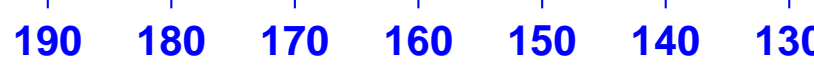

120

11010090

80

70

60

50

40

30

20

10

ppm 
5,6-Diphenyl-3-[6-(5-phenyl-2H-pyrazol-3-yl)-pyridin-2-yl] $[1,2,4]$ triazine $(9)$

VGB-B-19(5)

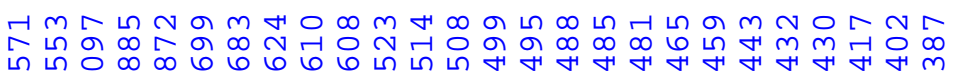

ת

N1,

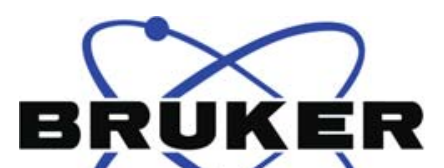

(x)

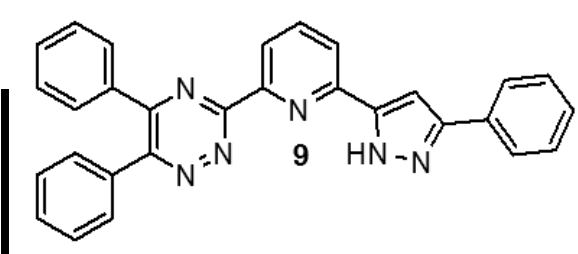

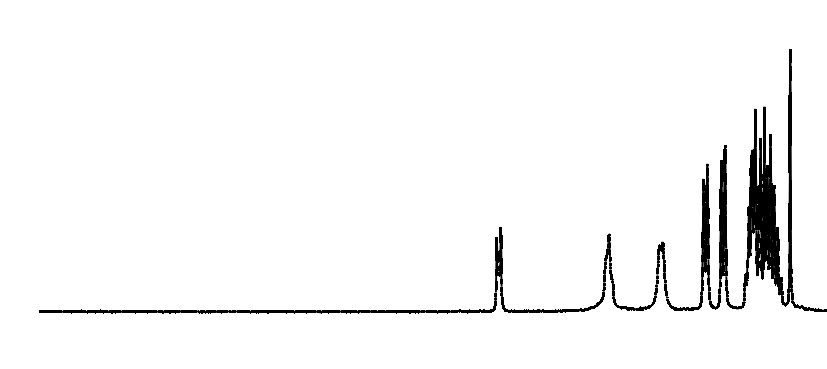

10

9

8

7

6

5

4

3

2

1

ppm

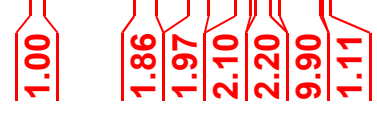


5, 6-Diphenyl - 3 - [6 - (5 - phenyl-2H-pyrazol-3-yl) - pyridin-2-yl] $[1,2,4]$ triazine $(9)$ VGB-B-19(5)
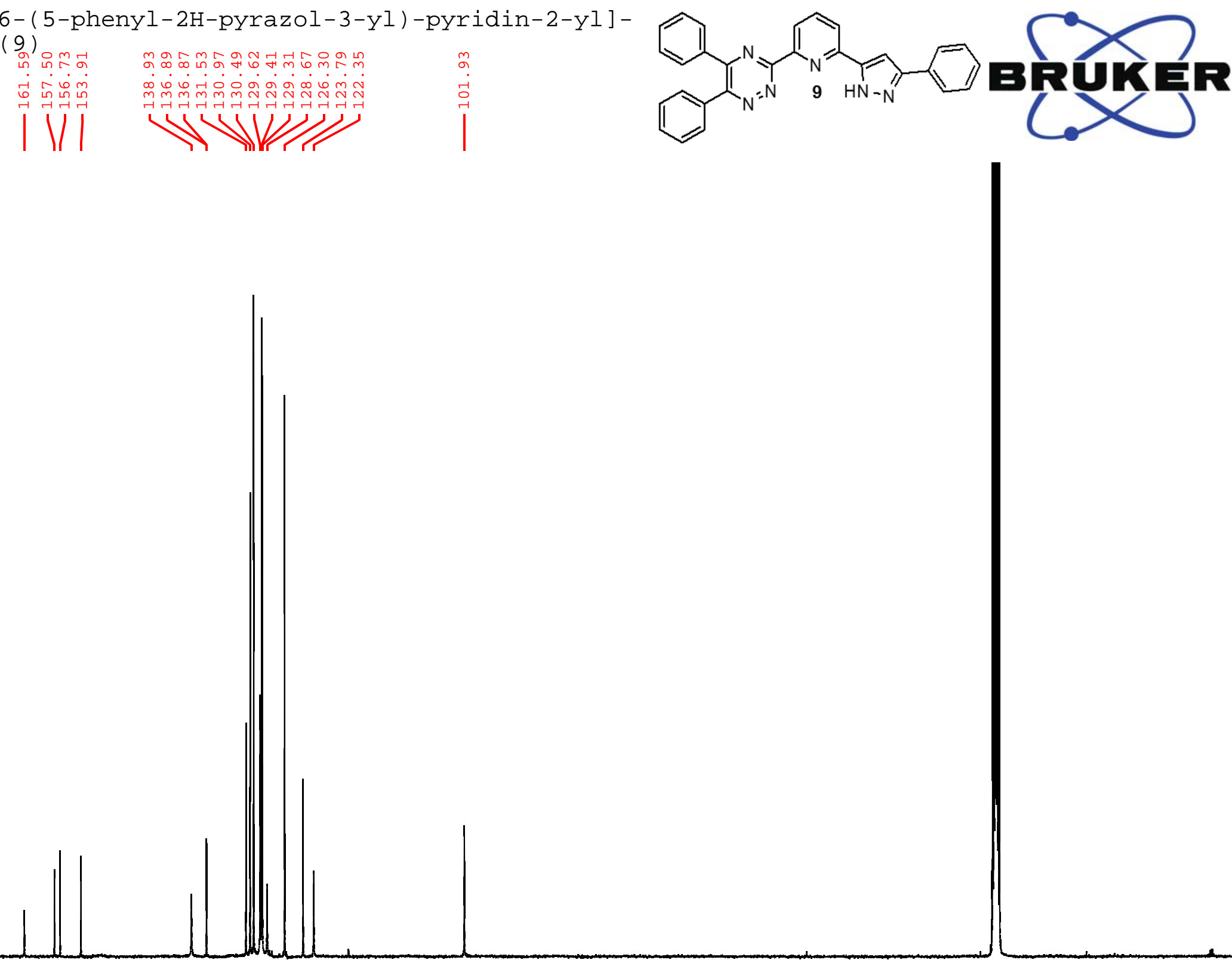
5,6-Diphenyl-3- [6-(5-p-tolyl-2H-pyrazol-3-yl) - pyridin-2-yl] $[1,2,4]$ triazine (2) VGB-A-283(15)

mo

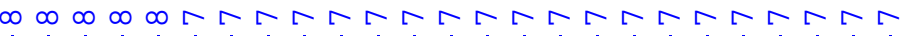
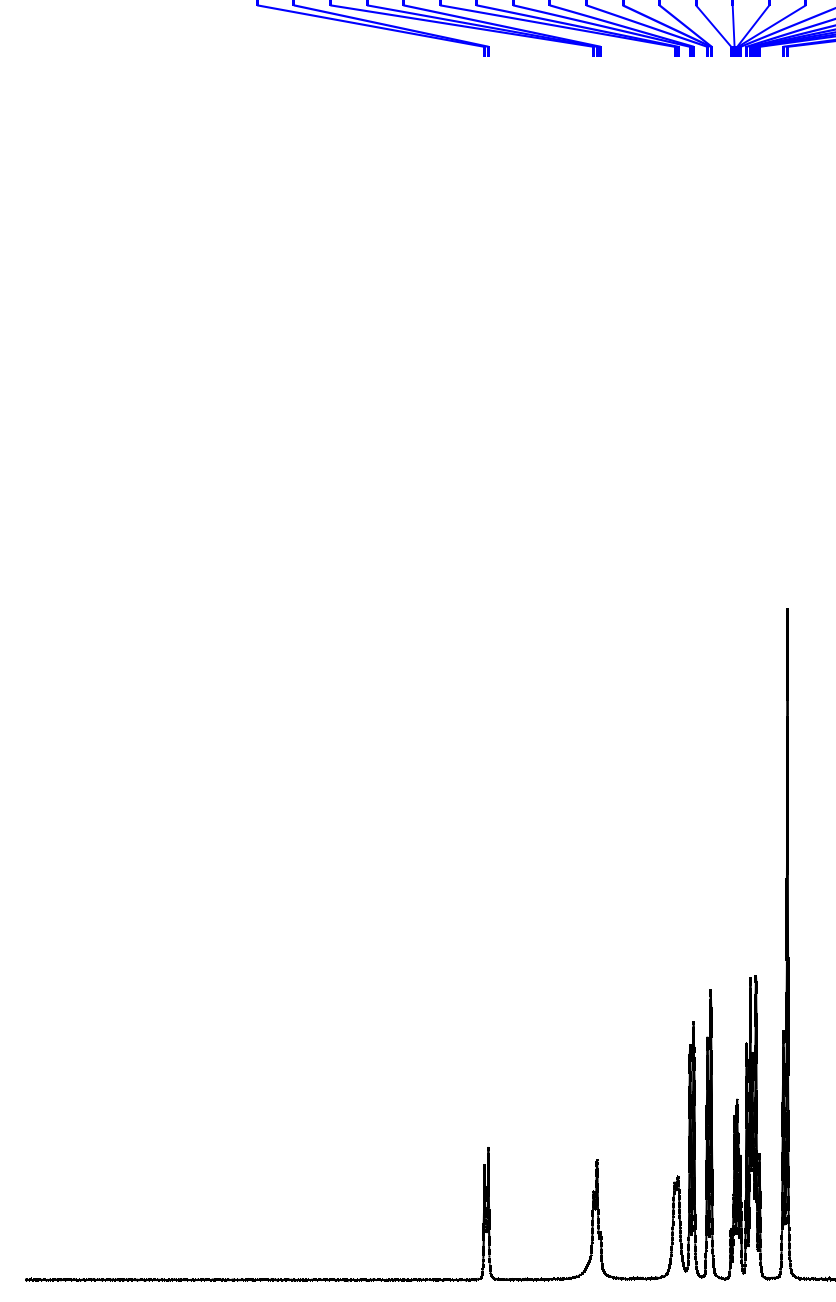

10 8

7

6

5

4

3

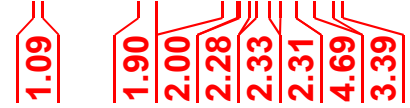

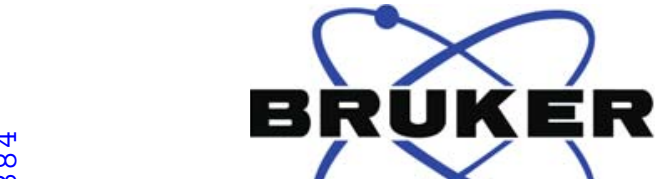

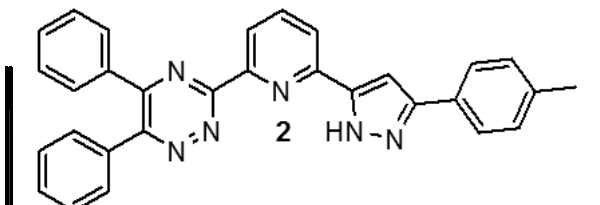


5,6-Diphenyl-3-[6-(5-p-tolyl-2H-pyrazol-3-yl)-pyridin-2-yl] $[1,2,4]$ triazine $(2)$ VGB-B-283(15)
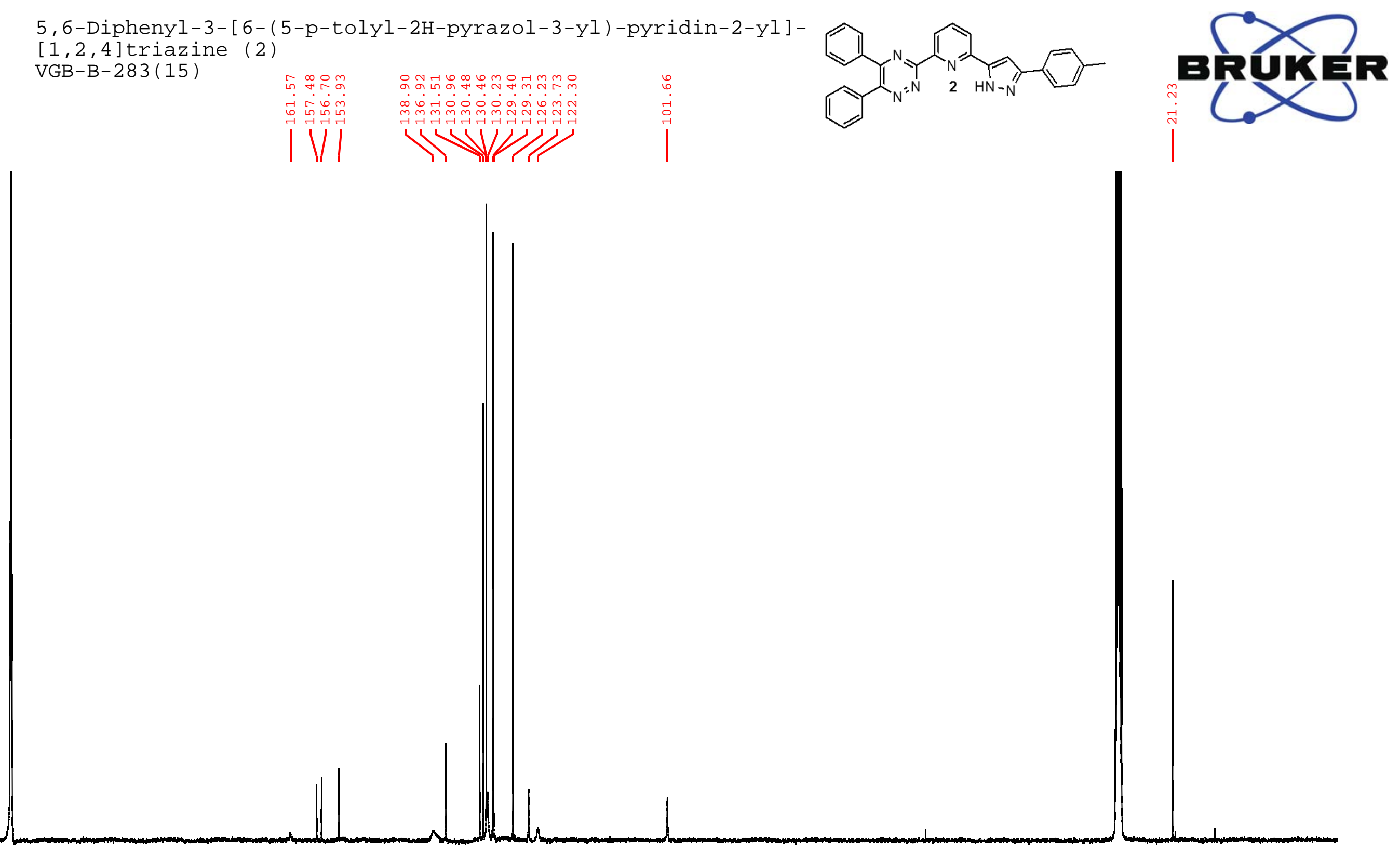
5,6-Diphenyl-3-[6-(3-p-tolyl-1H-pyrazol-4-yl)-pyridin-2-yl] $[1,2,4]$ triazine

VGB-C-31(1)

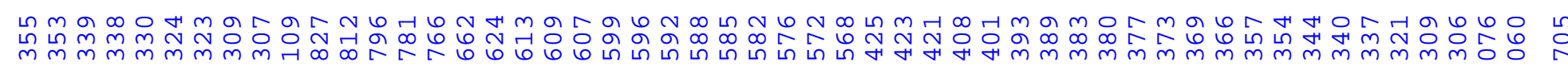
舟向
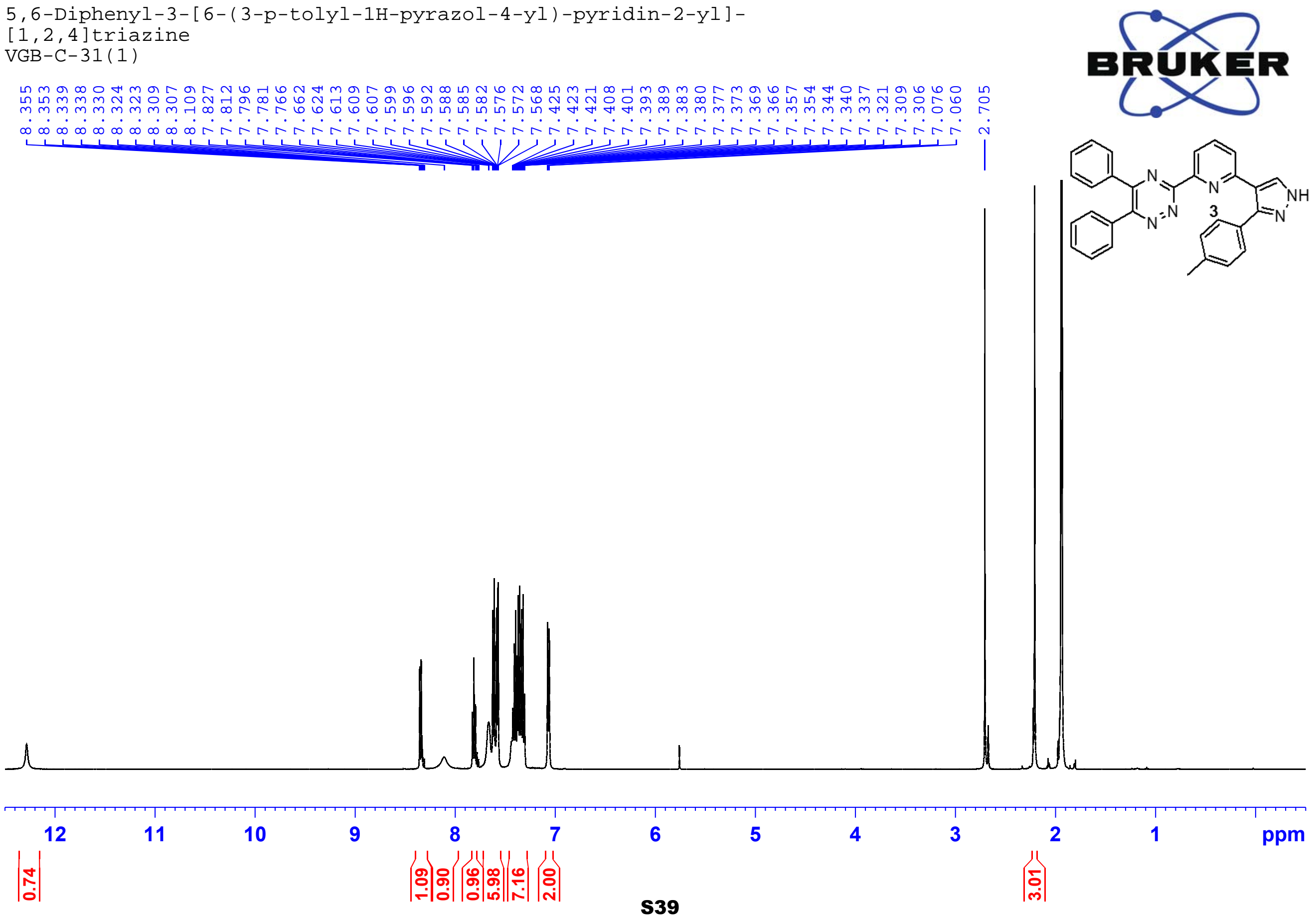
5,6-Diphenyl-3- [6- (3-p-tolyl-1H-pyrazol-4-yl) -pyridin-2-yl] $[1,2,4]$ triazine VGB-C-31(1)

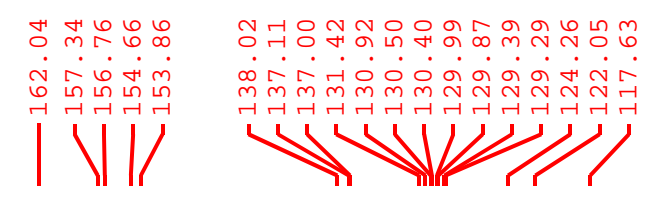

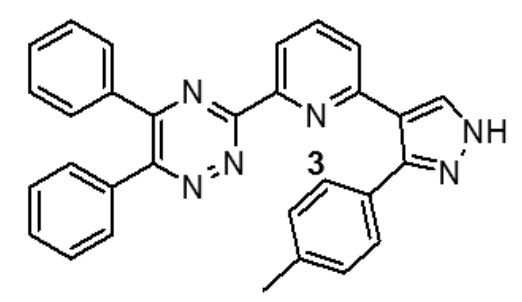

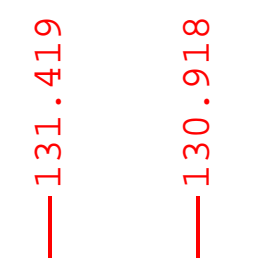

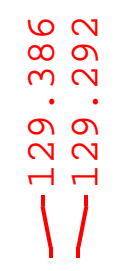

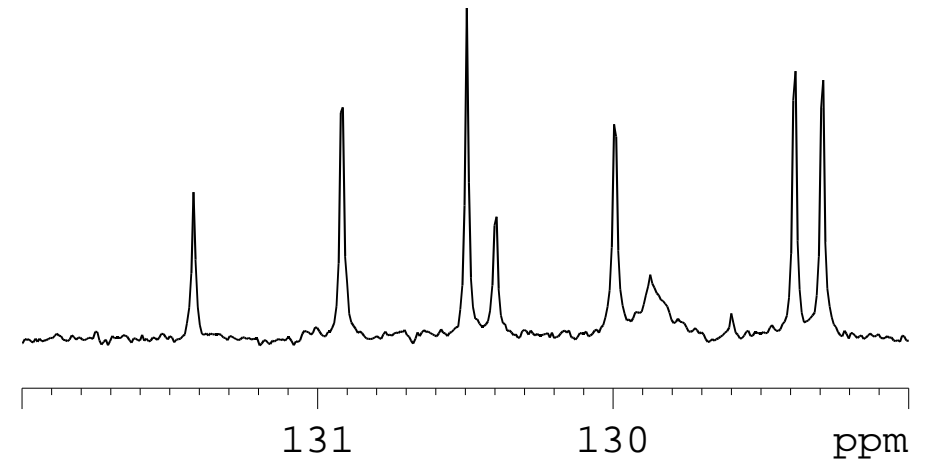

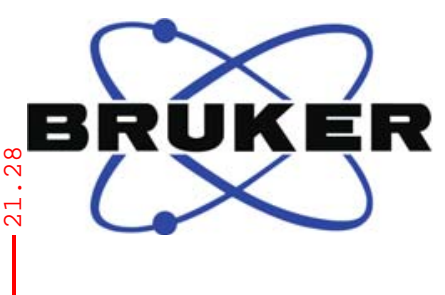

BRURER<smiles>C1CCCC1</smiles> 
3-\{6- [5- (4-Fluoro-phenyl) - 2H-pyrazol-3-yl] - pyridin-2-yl\}-5,6-diphenyl$[1,2,4]$ triazine $(10)$

VGB-B-225(5)

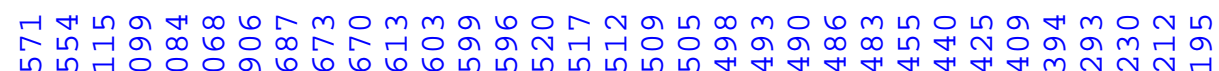

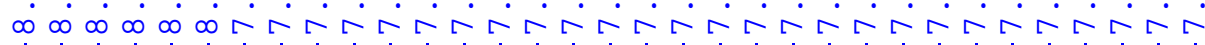

en $(x)$

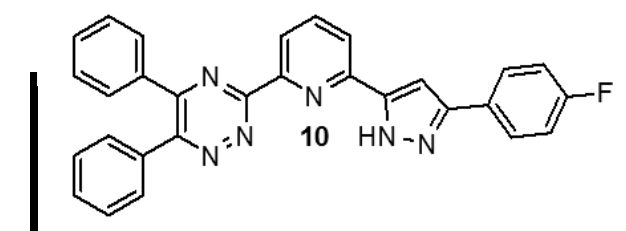

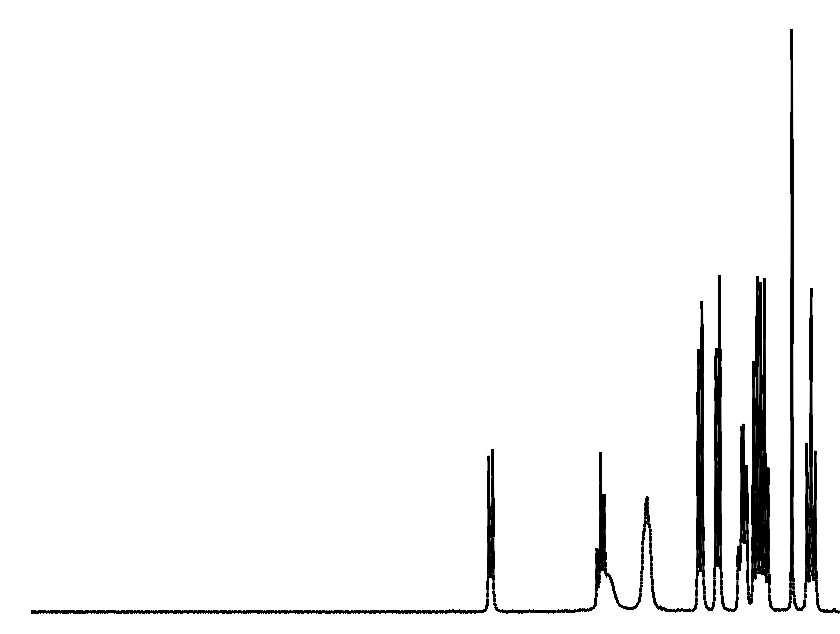

10

9

8

7

6

5

4

3

2

1

ppm

(L) 
VGB-B-251( 5 )

5,6-Diphenyl-3-\{6-[5-(4-trifluoromethyl-phenyl)-2H-pyrazol-3-yl] pyridin-2-yl\}-[1,2,4]triazine (11)

ஓ

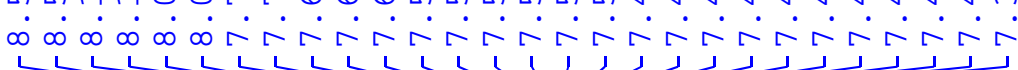

aruKer $(1)$

M

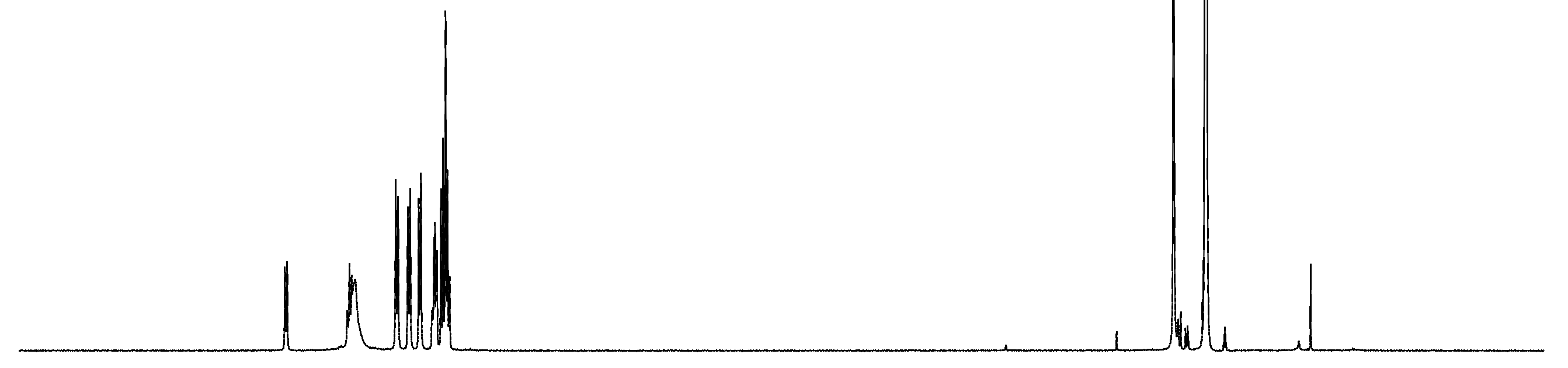


5, 6-Diphenyl-3-\{6-[5-(4-trifluoromethyl-phenyl) - $2 \mathrm{H}$-pyrazol-3-yl] pyridin-2-yl\}-[1,2,4] triazine (11) VGB-B-251(5)
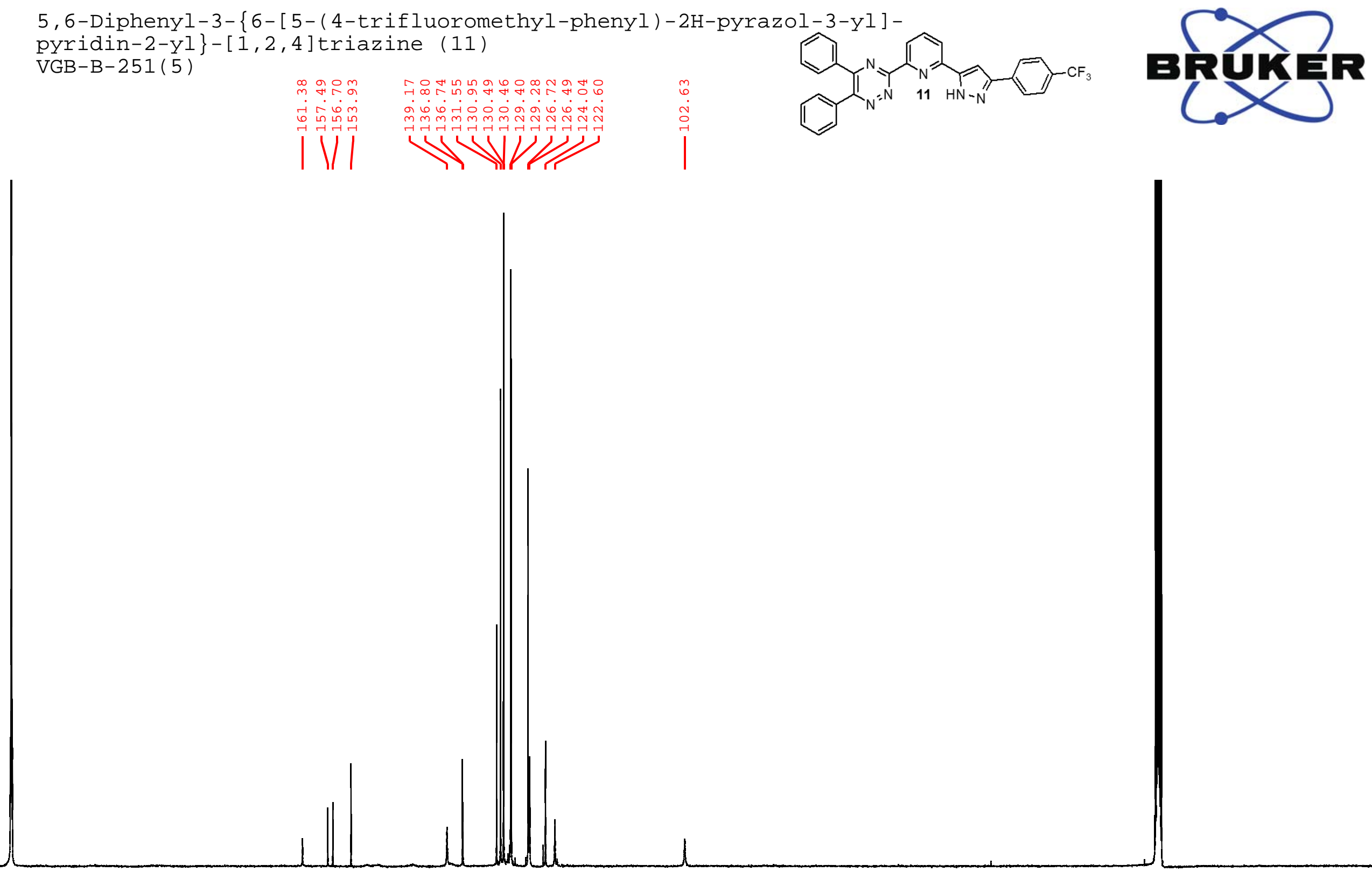
3 - $\{6$ - [5 - (4 - Bromo - phenyl) - $2 \mathrm{H}-$ pyrazol-3-yl] - pyridin-2-yl $\}$ 5,6 -diphenyl- $[1,2,4]$ triazine (12)

VGB-B-243(5)

مo

o

1

$\longrightarrow$ m

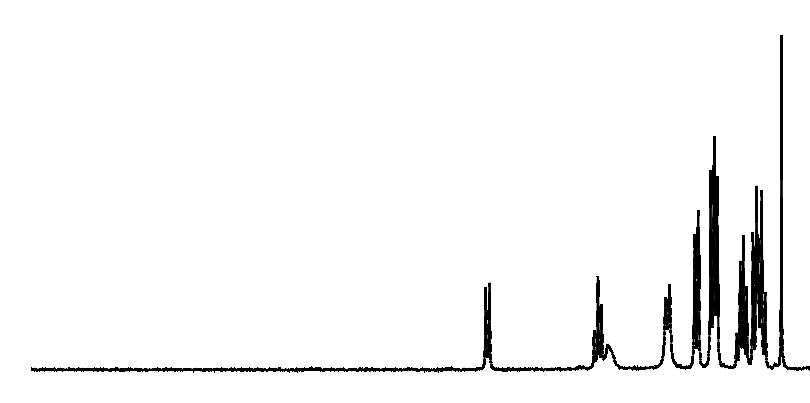

10

9

8

7

6

5

45

3

2

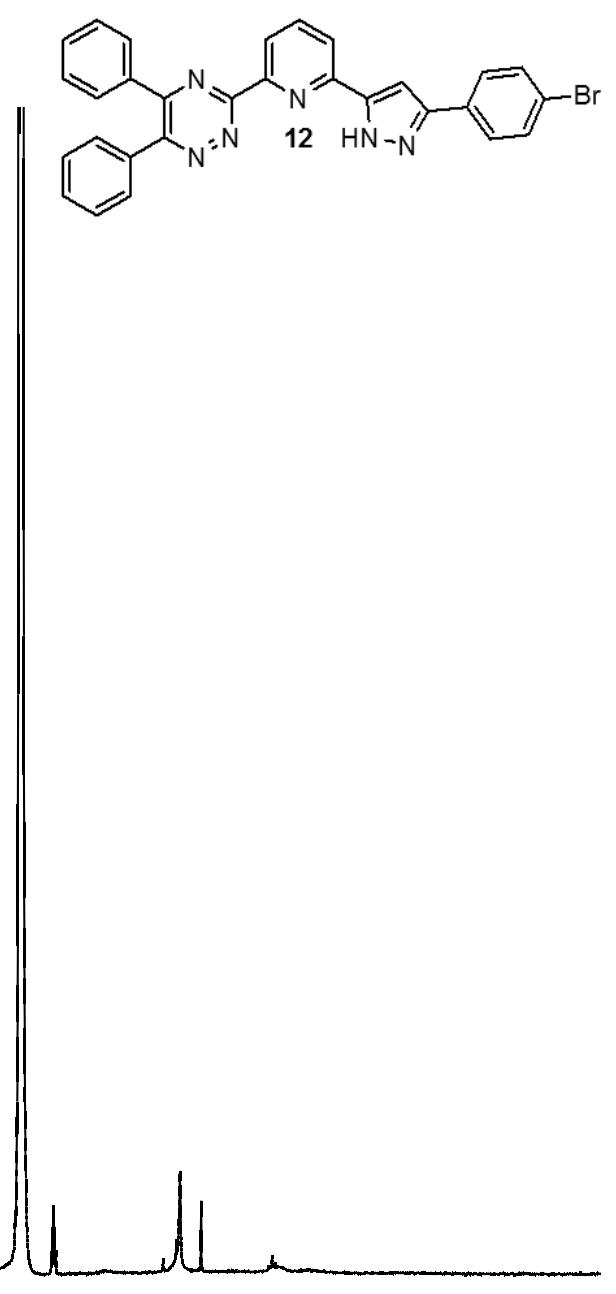

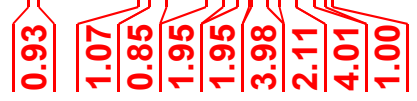


3 - $\{6$ - [5- (4-Bromo - phenyl) - 2H-pyrazol-3-yl] - pyridin-2-yl $\}-5,6$-diphenyl$[1,2,4]$ triazine $(12)$

VGB-B-243(15)
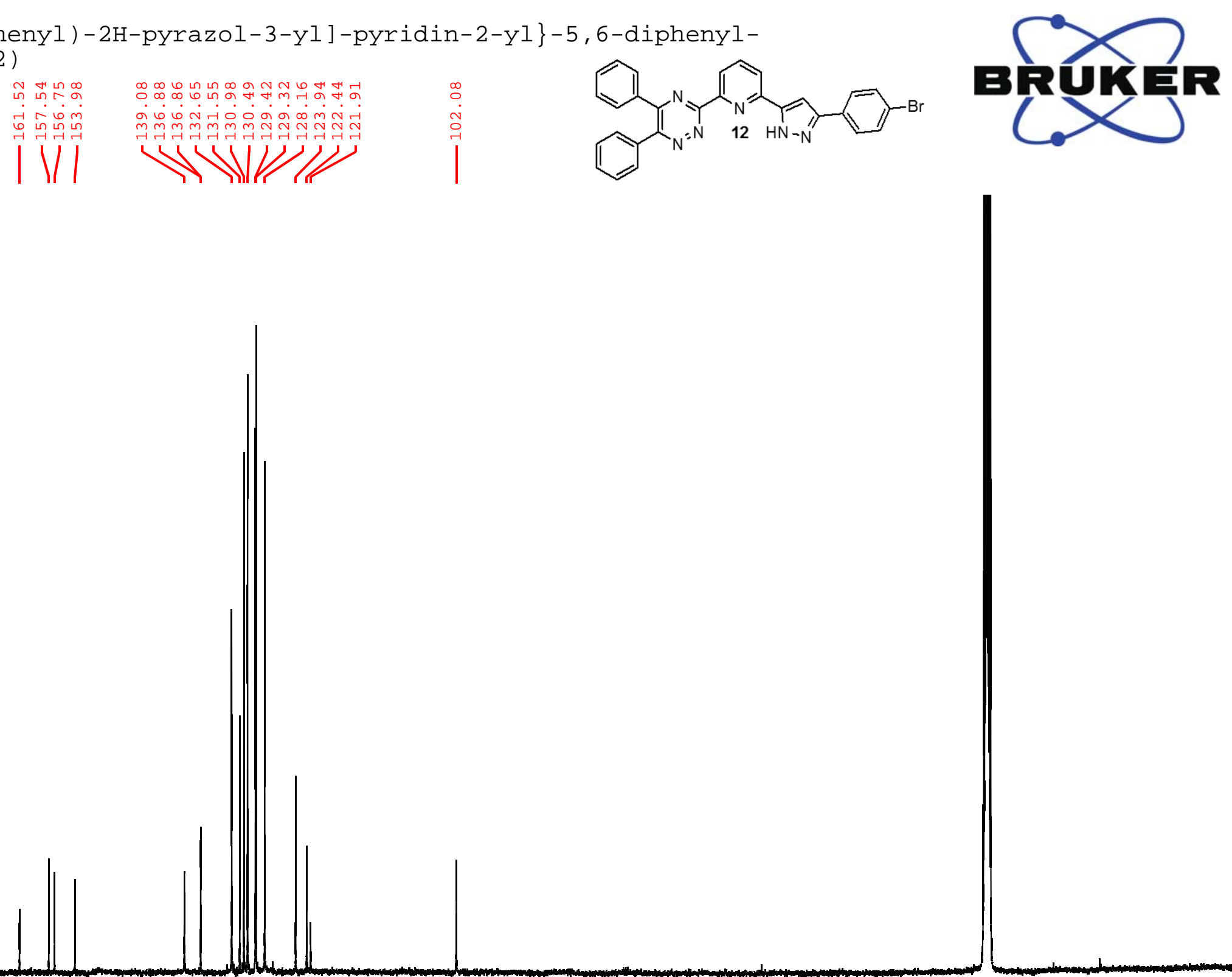

200

170160

150

140130

120

$\begin{array}{lll}110 & 100 & 90\end{array}$

80

70

60

50

40

30

20

10

ppm 
(4-\{5-[6-(5,6-Diphenyl- [1,2,4]triazin-3-yl) -pyridin-2-yl]-1Hpyrazol-3-yl\}-phenyl) - dimethylamine (13)

VGB-C-17(5)

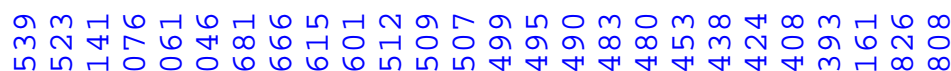

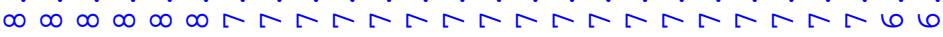
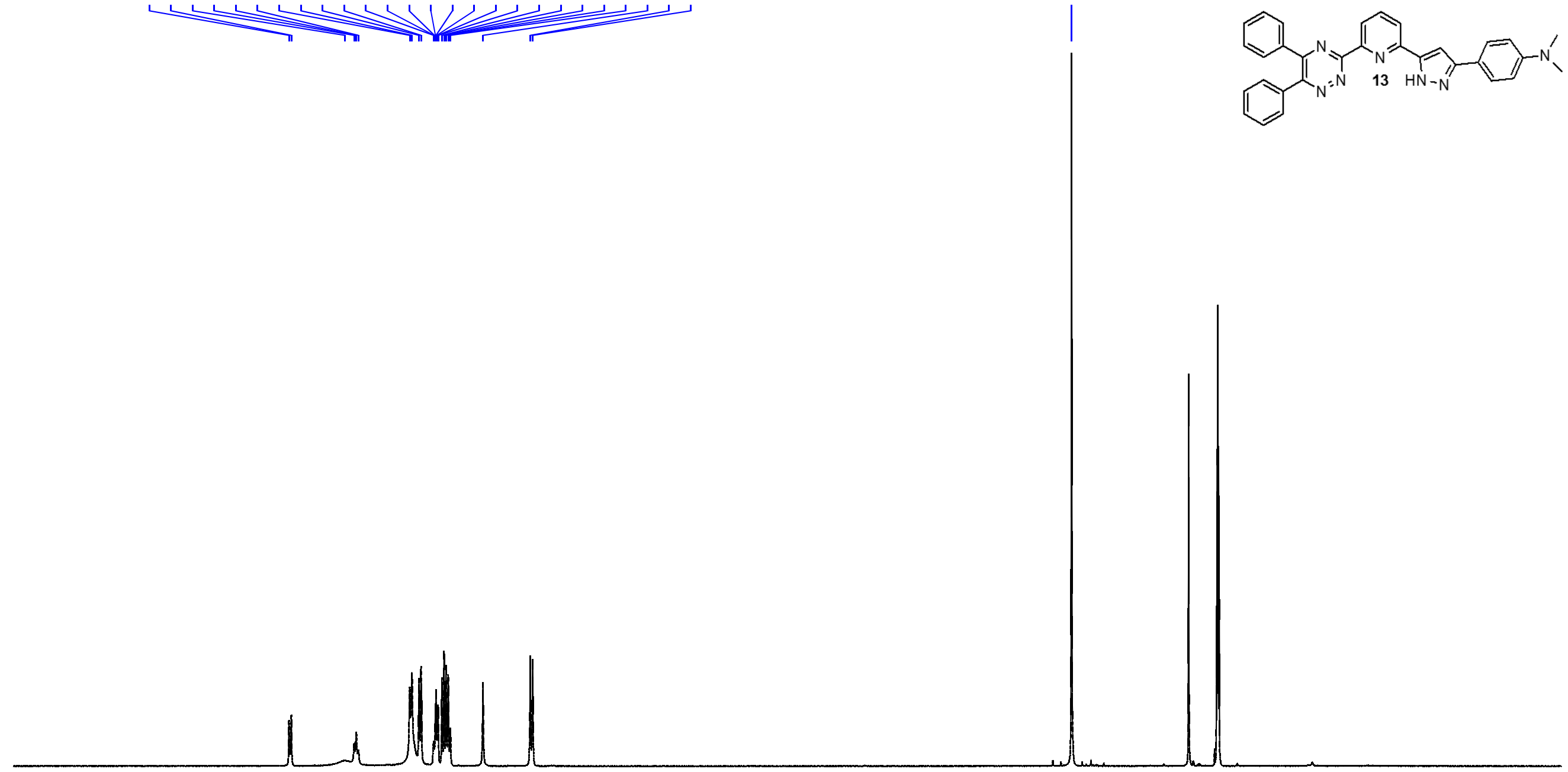
(4- $\{5$ - [6- (5,6-Diphenyl- $[1,2,4]$ triazin-3-yl) - pyridin-2-yl] - $1 \mathrm{H}$ pyrazol-3-yl\}-phenyl)-dimethylamine (13)

VGB-C-17(5)
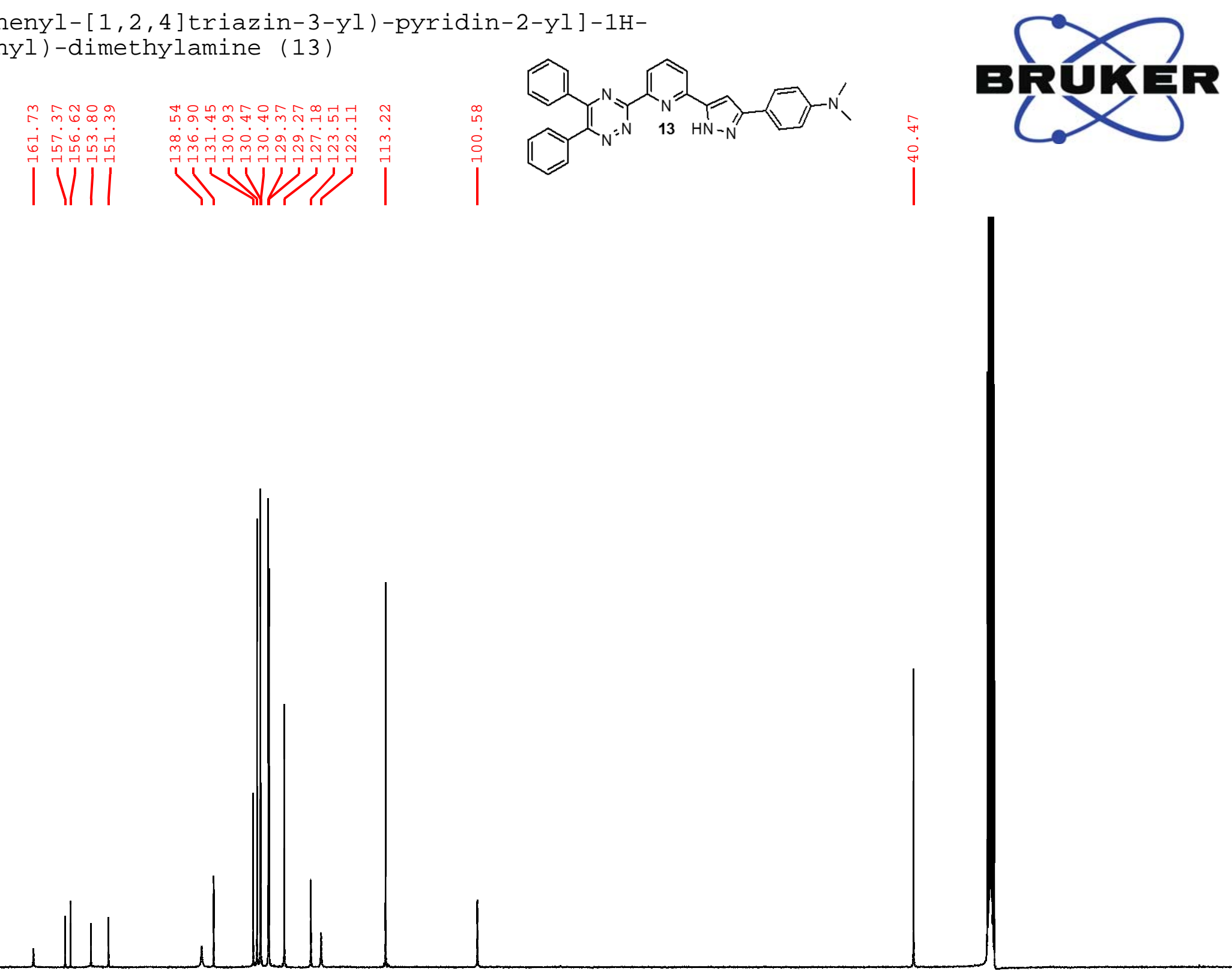

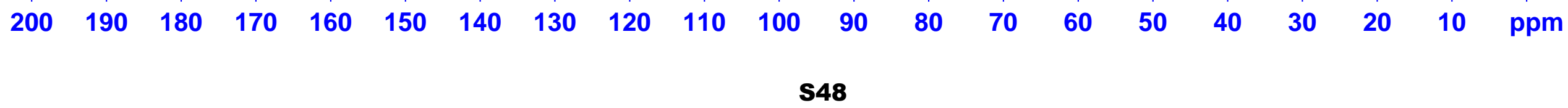


3 - $\{6$ - [5 - (4 - Methoxy - phenyl) - 2H-pyrazol-3-yl] - pyridin-2-yl $\}-5,6$ diphenyl- $[1,2,4]$ triazine (14)

VGB-B-203(5)

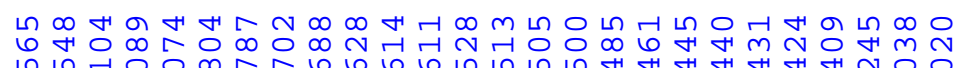

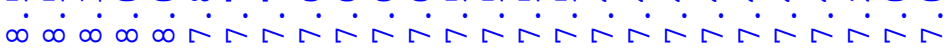

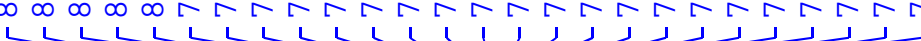

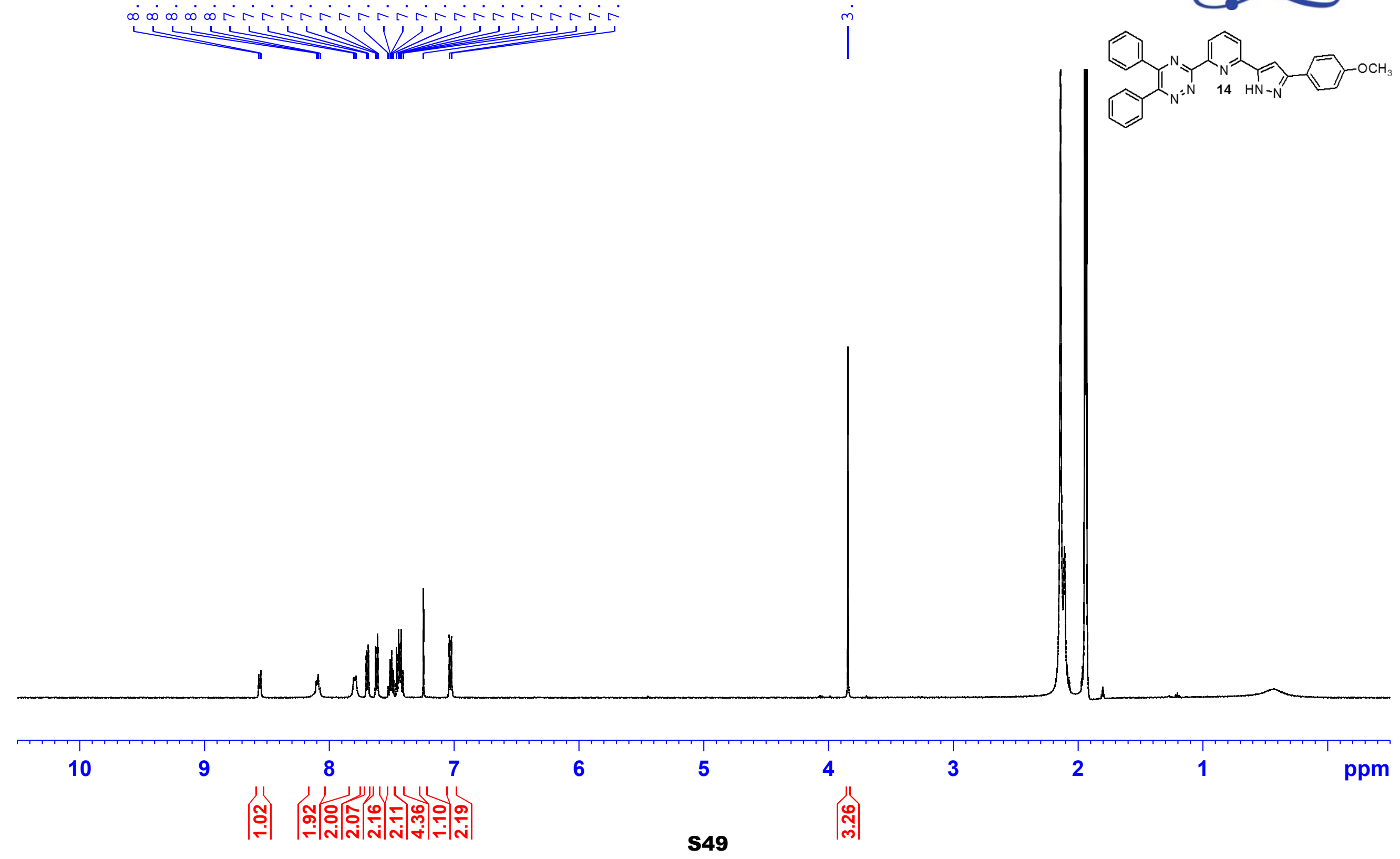


3-\{6- [5- (4-Methoxy-phenyl) - 2H-pyrazol-3-yl] - pyridin-2-yl\}-5,6diphenyl-[1,2,4]triazine (14)

VGB-B-203(5)
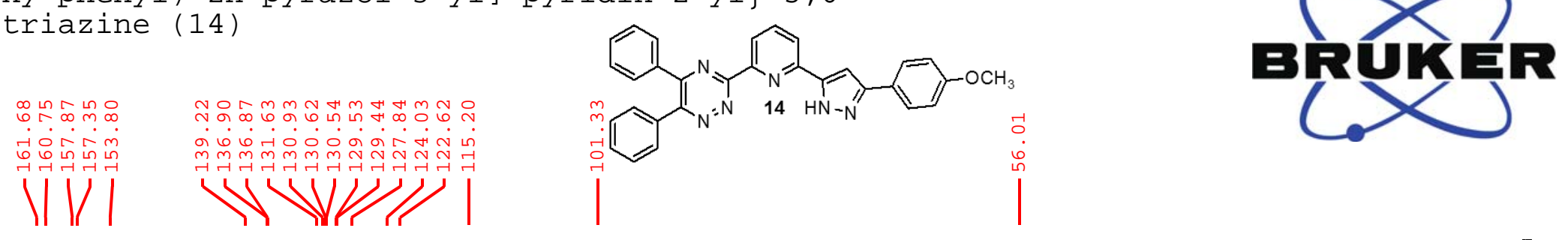
3 - $\{5$ - [6- (5,6-Diphenyl - [1,2,4]triazin-3-yl) - pyridin-2-yl]-1H-pyrazol-3-yl $\}$ benzoic acid methyl ester (15) VGB-B-215(15)

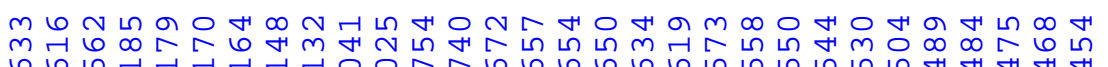

$\stackrel{\odot}{\sim}$

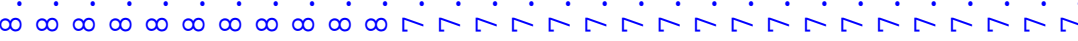

A

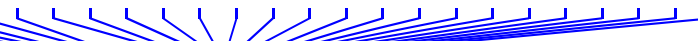

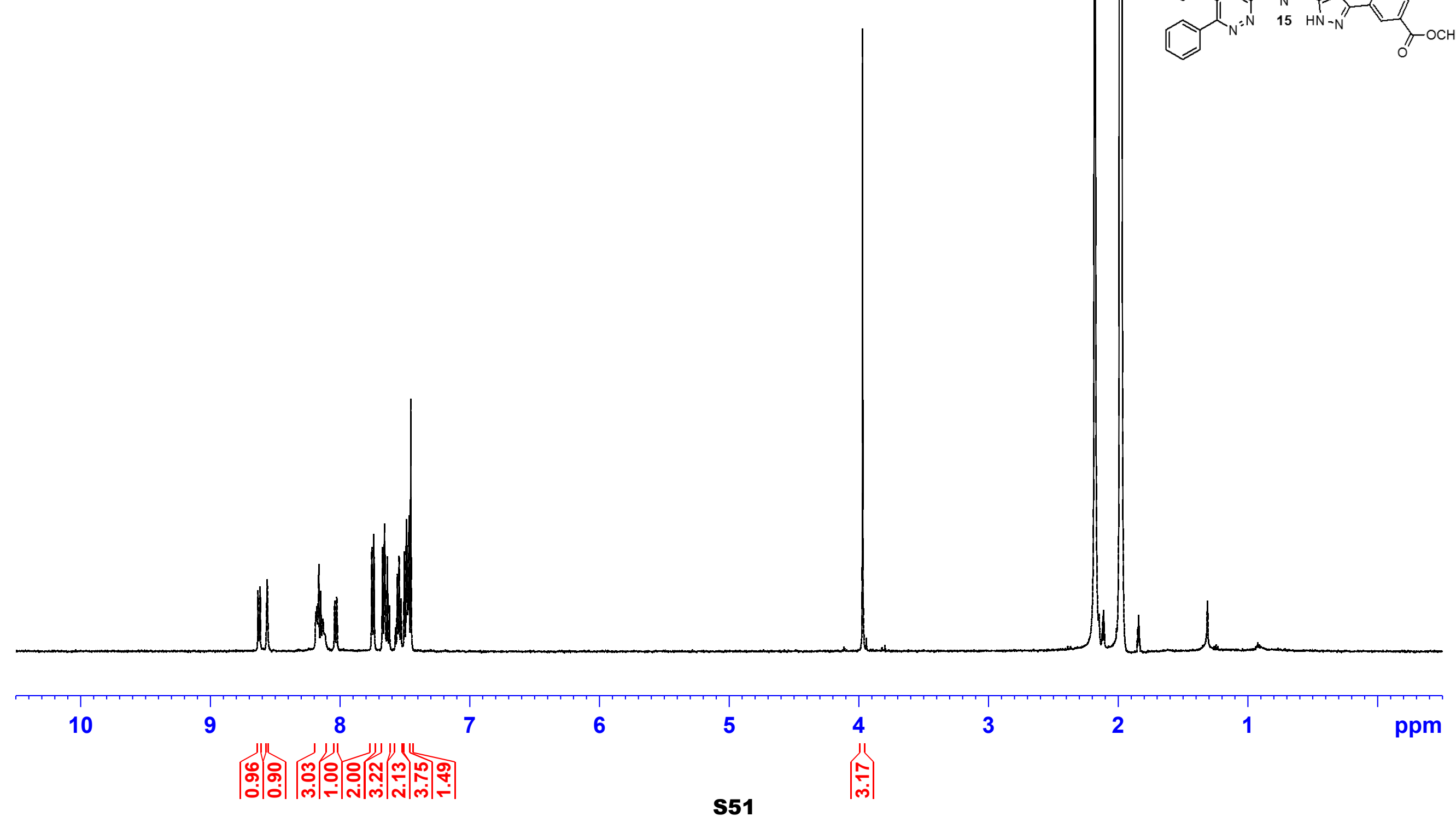


$3-\{5$ - [6- (5,6-Diphenyl- $[1,2,4]$ triazin-3-yl) - pyridin-2-yl] - $1 \mathrm{H}$-pyrazol-3-yl $\}$ benzoic acid methyl ester (15)

VGB-B-215(15)

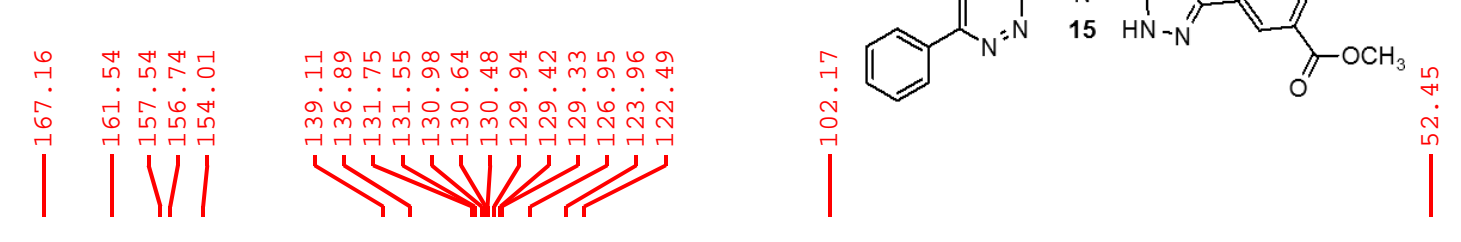

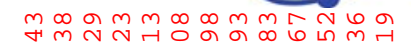

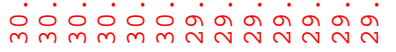

WWIII)
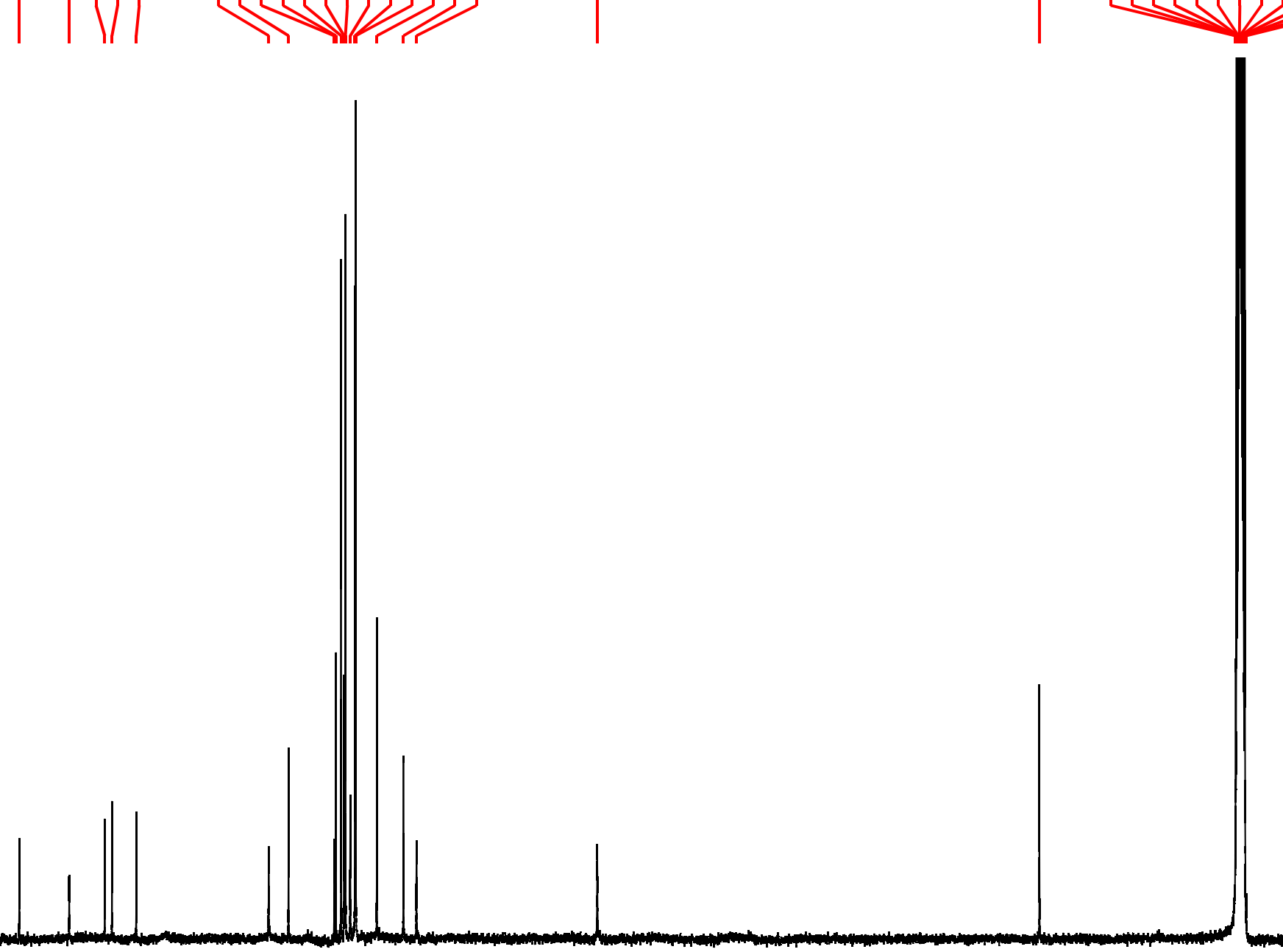

$\begin{array}{lllllllllllllllllllll}200 & 190 & 180 & 170 & 160 & 150 & 140 & 130 & 120 & 110 & 100 & 90 & 80 & 70 & 60 & 50 & 40 & 30 & 20 & 10 & \mathrm{ppm}\end{array}$


3 - $\{6$ - [5- (2-Iodo - phenyl) - 2H-pyrazol-3-yl] - pyridin-2-yl $\}$-5, 6-diphenyl$[1,2,4]$ triazine $(16)$

VGB-B-249(5)

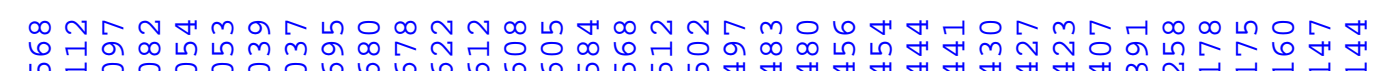

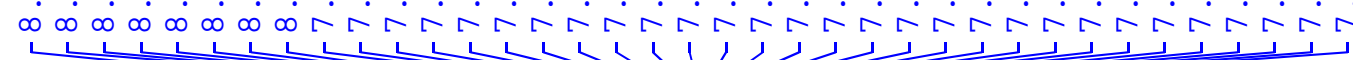

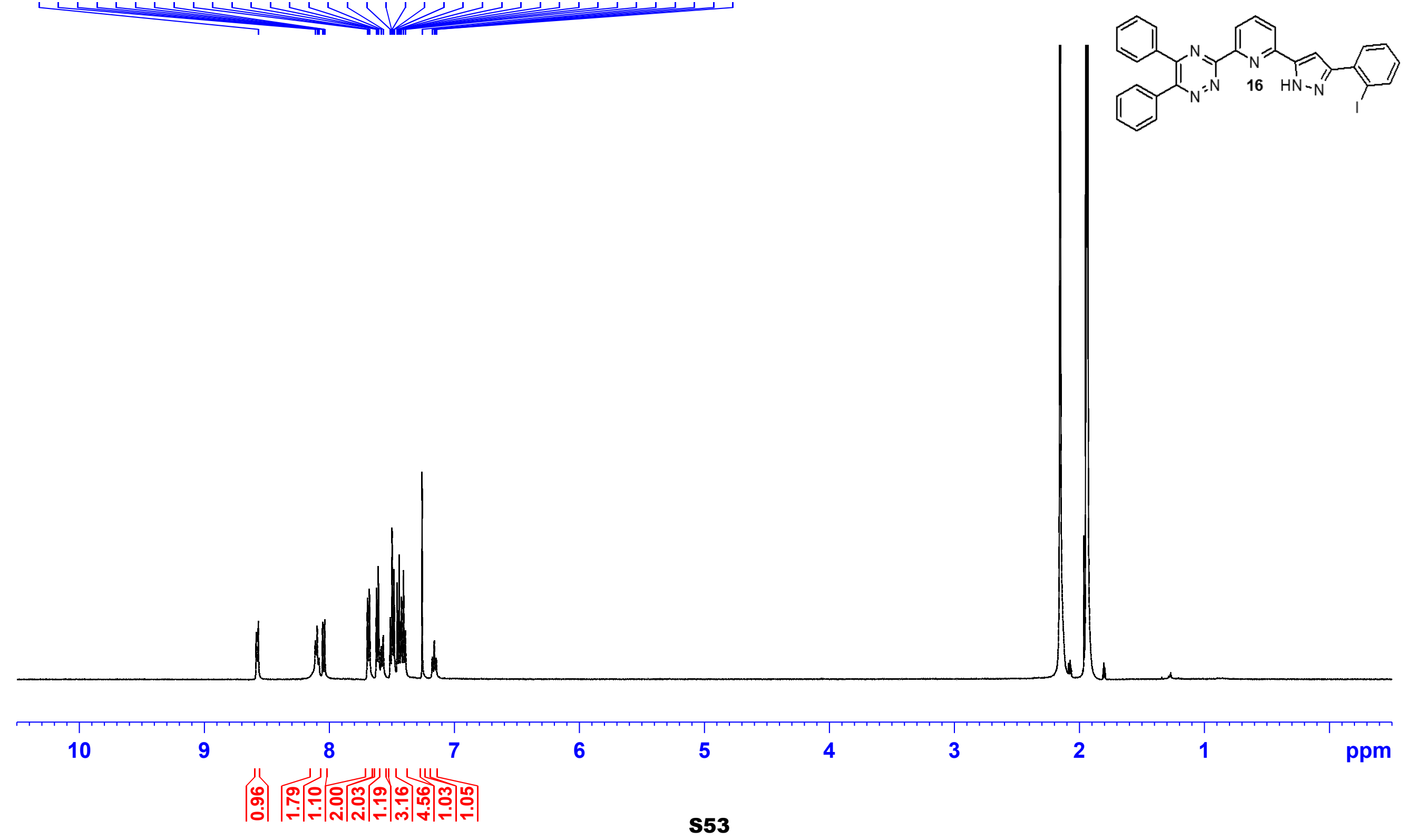

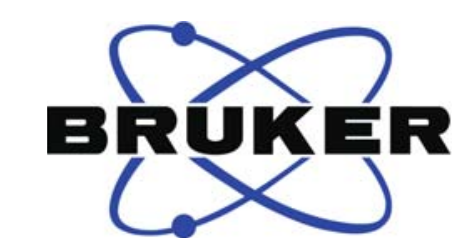

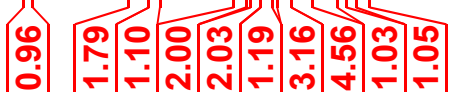


5,6-Diphenyl-3-\{6-[5-(2,4,6-trifluoro-phenyl)-2H-pyrazol-3-yl] pyridin-2-yl\}-[1,2,4]triazine (17)

VGB-B-61(5)

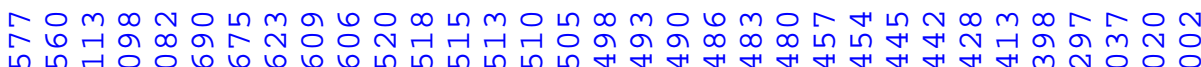

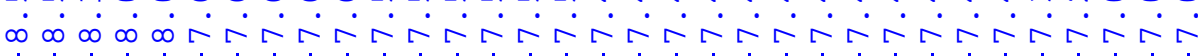

Be ,

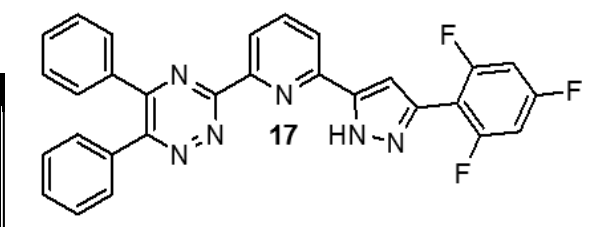

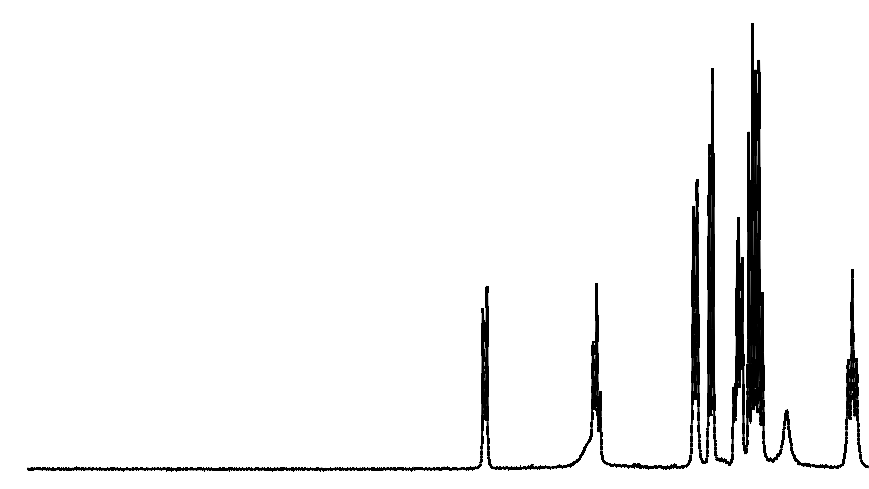

10

9 8

7

6

5

4

3

2

1

ppm

| 
5,6-Diphenyl-3-\{6-[5-(2,4,6-trifluoro-phenyl) - 2H-pyrazol-3-yl] pyridin-2-yl\}-[1,2,4]triazine (17)

VGB-B-61(5)

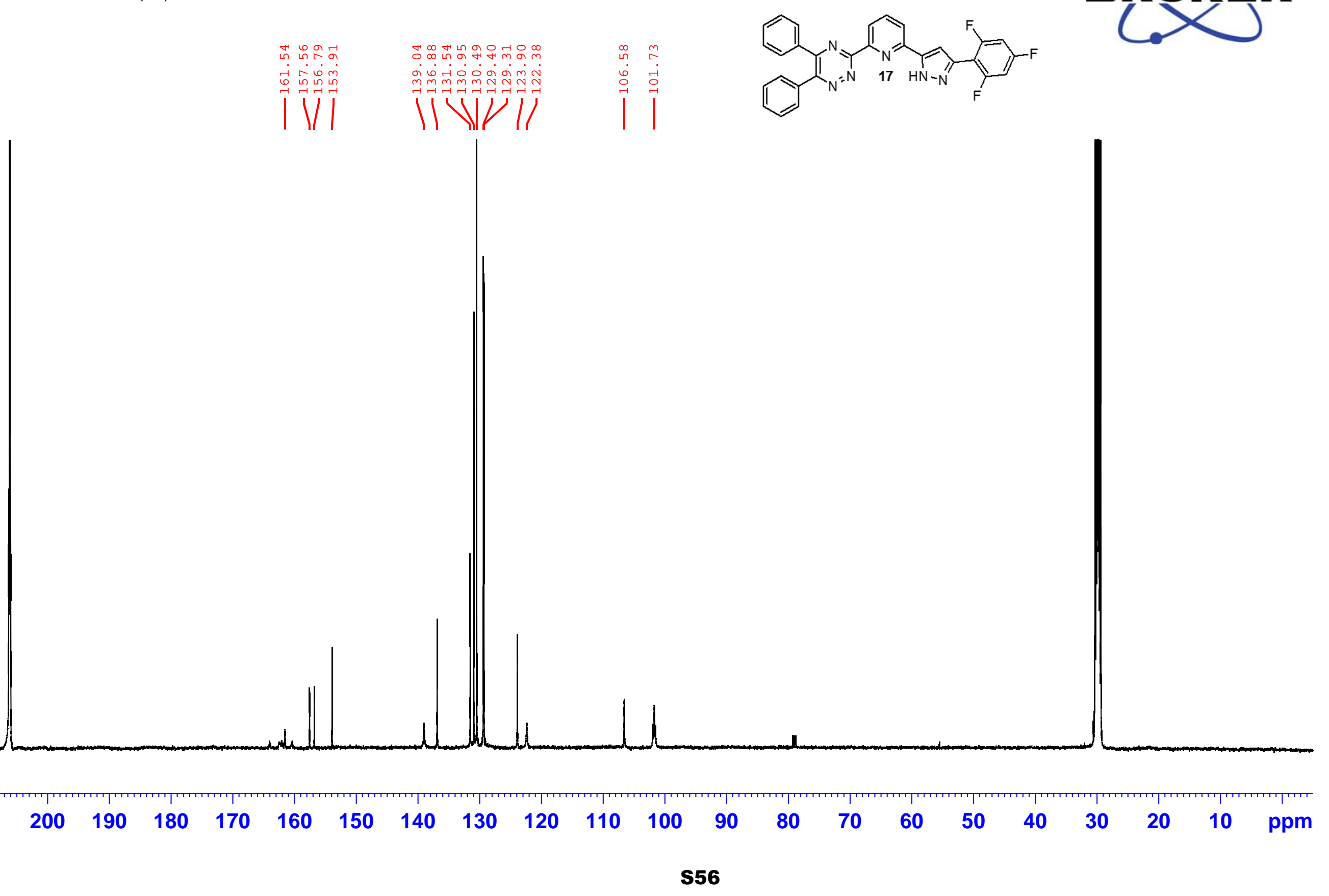


3 - $\{6$ - [5- (3,5-Di - tert - butyl-phenyl) - 2H-pyrazol-3-yl] - pyridin-2-yl $\}$ 5,6 -diphenyl- $[1,2,4]$ triazine (18)

VGB-B-211(15)

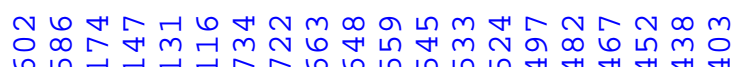

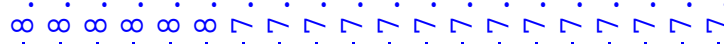

$N_{m}^{2}$
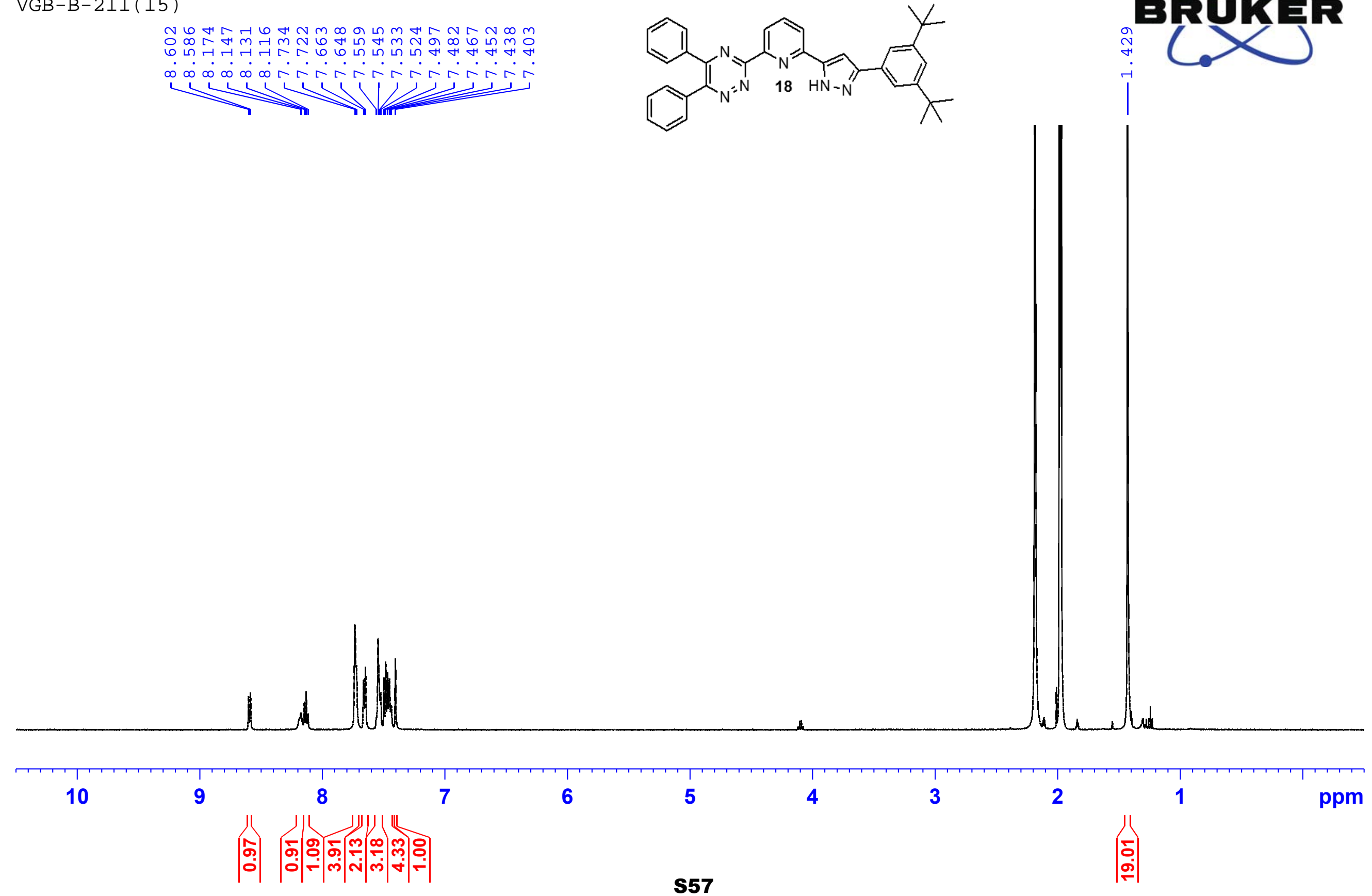
5,6-Diphenyl-3- [6-(5-pyridin-4-yl-2H-pyrazol-3-yl)-pyridin-2-yl] $[1,2,4]$ triazine $(19)$

VGB-B-2019(15)

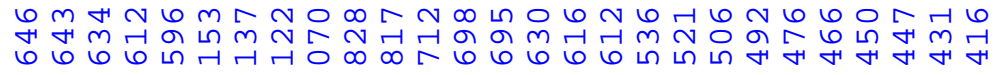

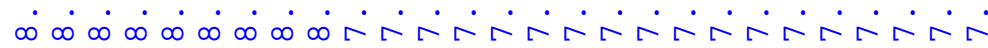

每

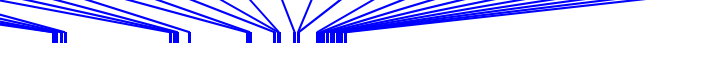

10

9 8

7

6

5

4

3

2

1

ppm స̦ 
5,6-Diphenyl-3- [6-(5-pyridin-4-yl-2H-pyrazol-3-yl) - pyridin-2-yl] $[1,2,4]$ triazine $(19)$

VGB-B-2019(15)
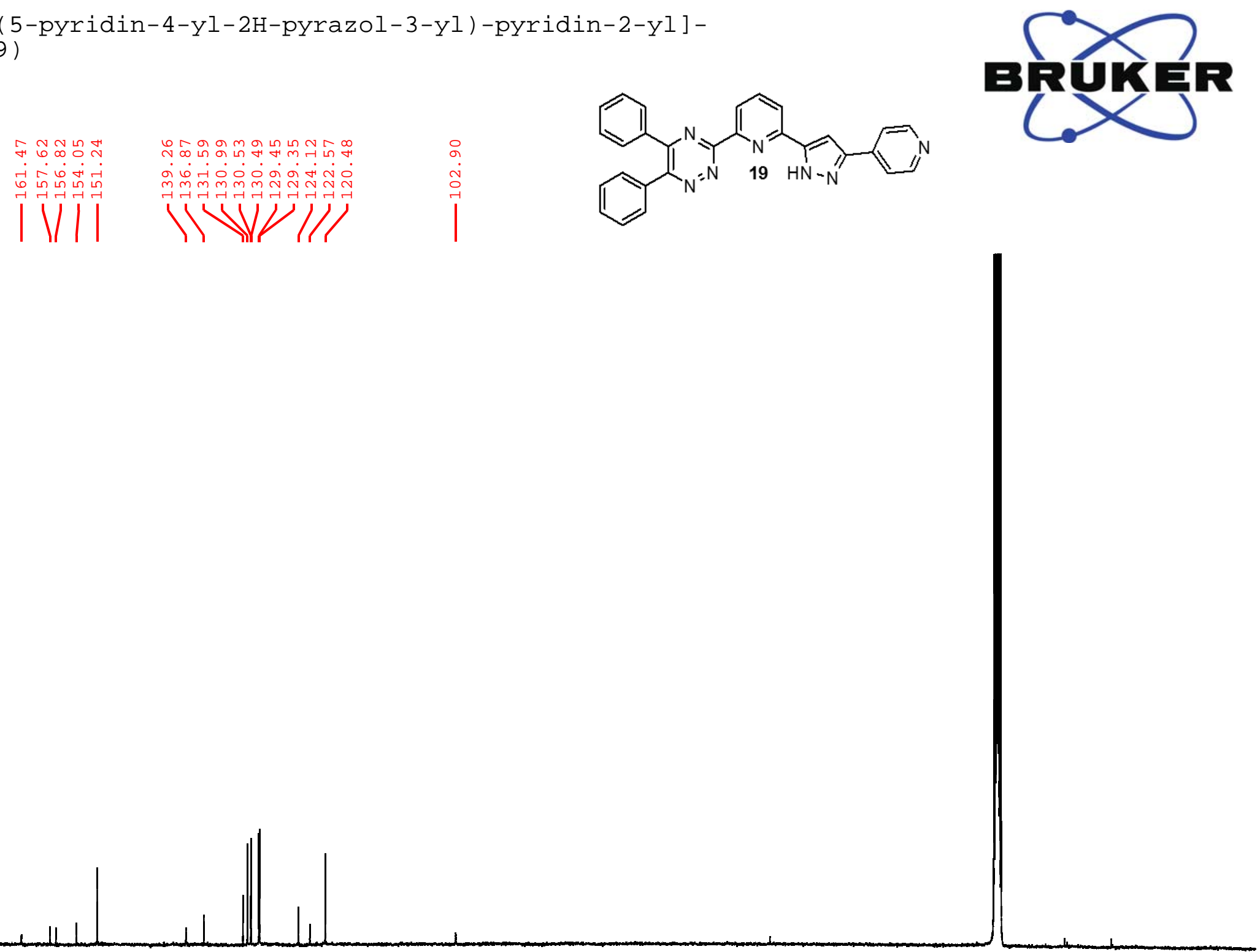

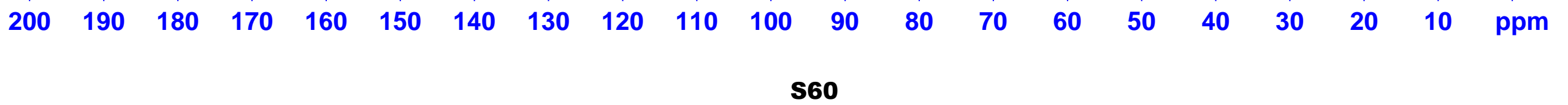


5,6-Bis - (4-cyclopropyl-phenyl) - 3- [6 - (5-phenyl-2H-pyrazol-3-yl) pyridin-2-yl]-[1,2,4]triazine (20)

VGB-B-265(4)

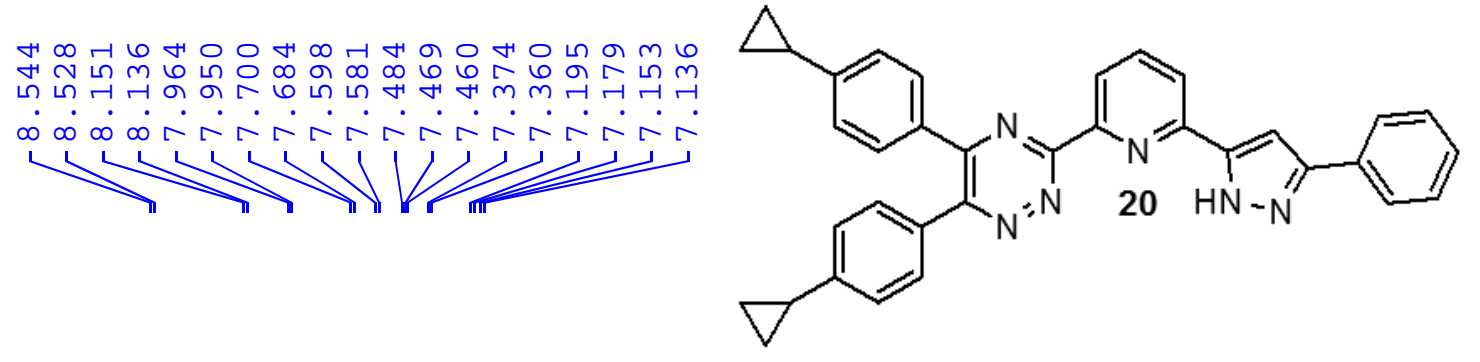

\section{ำ} ñंaें

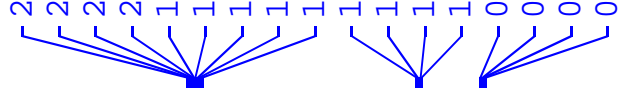

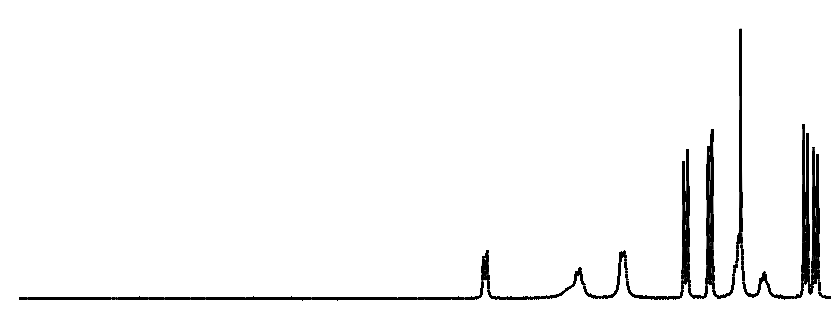

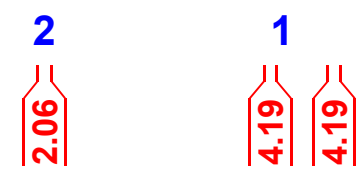


5,6-Bis - (4-cyclopropyl-phenyl) - 3- [6 - (5-phenyl-2H pyrazol-3-yl)-pyridin-2-yl]-[1, 2,4]triazine (20) VGB-B-265(15)

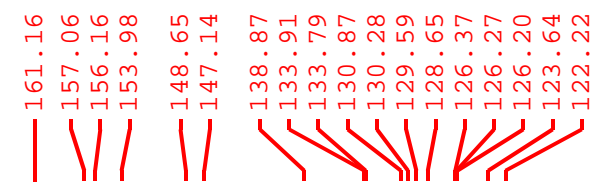

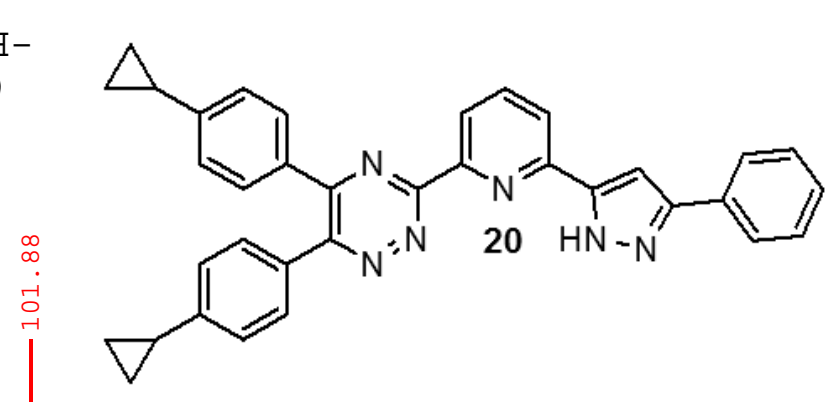
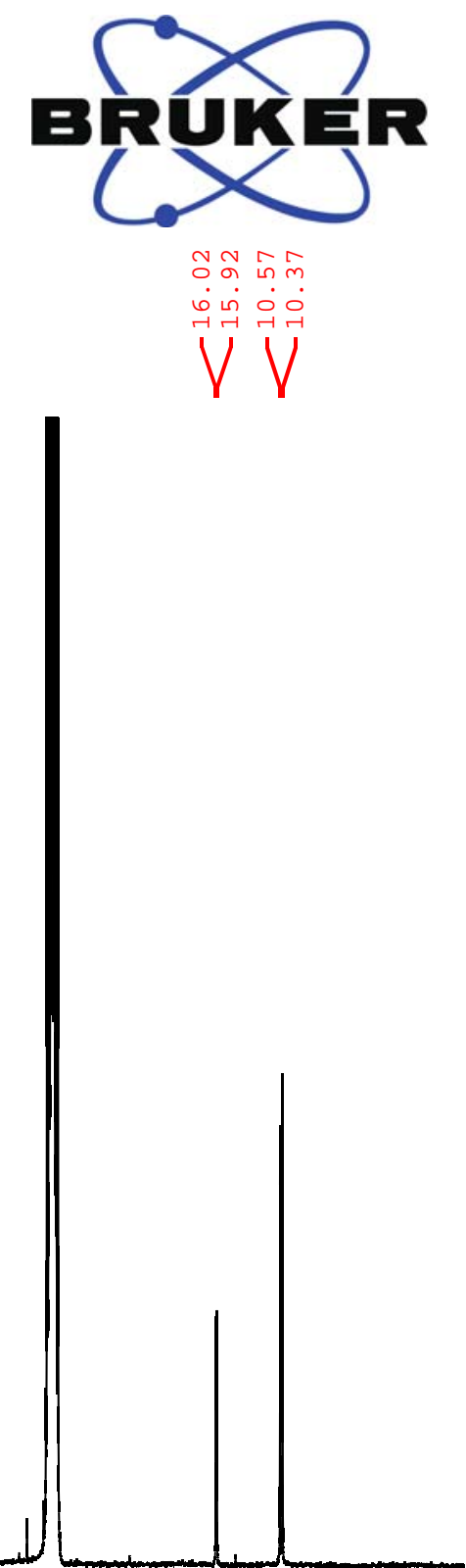
5,6-Bis - (4-cyclopropyl - phenyl ) - 3 - $\{6$ - [5 - (2 - iodo - phenyl) - $2 \mathrm{H}$ pyrazol-3-yl]-pyridin-2-yl\}-[1,2,4] triazine (21) VGB-B-269(5)

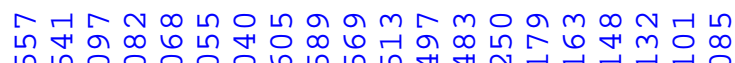
$\underbrace{\infty}_{\infty}$ $\underbrace{\infty}_{\text {III }}$

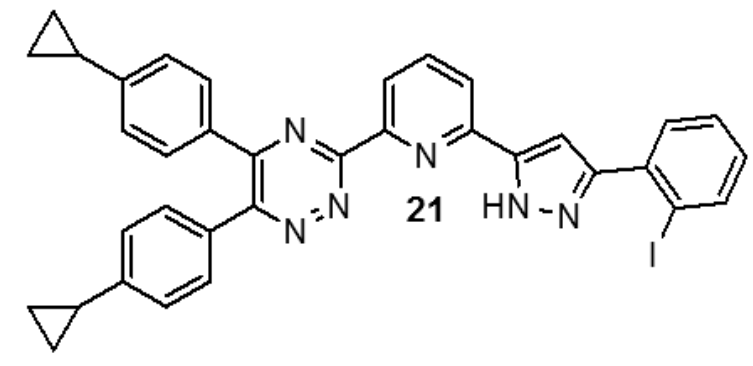

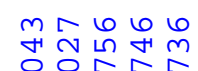
तिंबं०ं $\checkmark V$

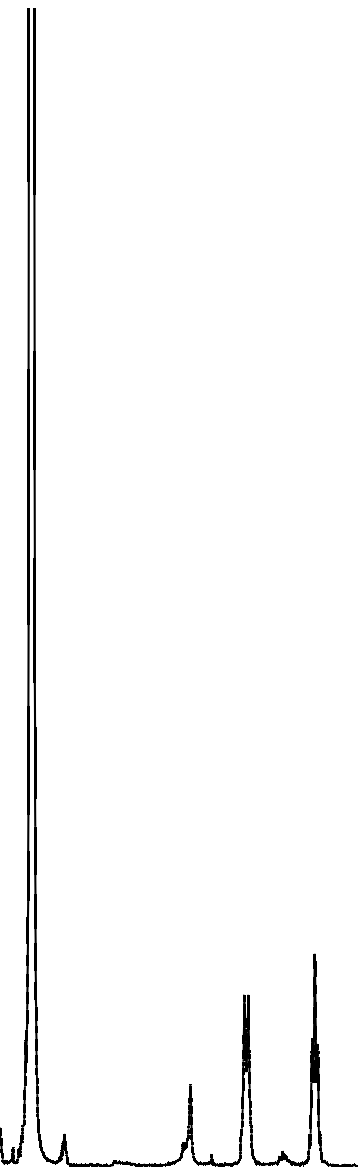

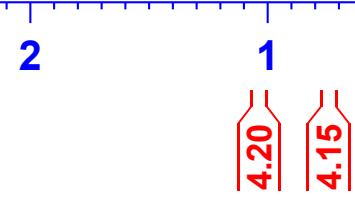


3 - $\{6$ - [5- (4-Bromo - phenyl) - $2 \mathrm{H}$ - pyrazol - 3-yl] - pyridin-2-yl $\}-5,6$ - bis (4-butyl-phenyl)-[1,2,4]triazine (22)

VGB-B-221(5)

オேオ・ !

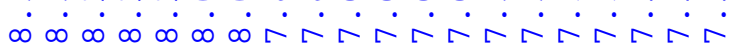
N1)

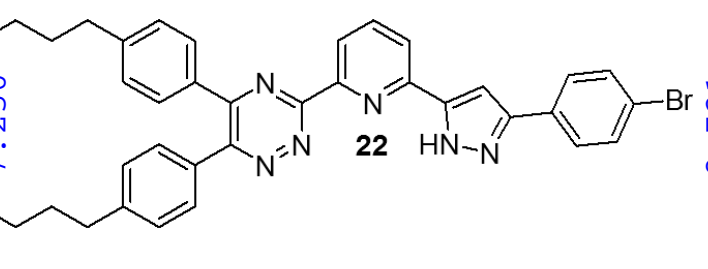
r

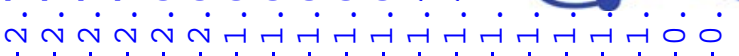
$1 / 21 / 1$<smiles>C1CCCCC1</smiles>

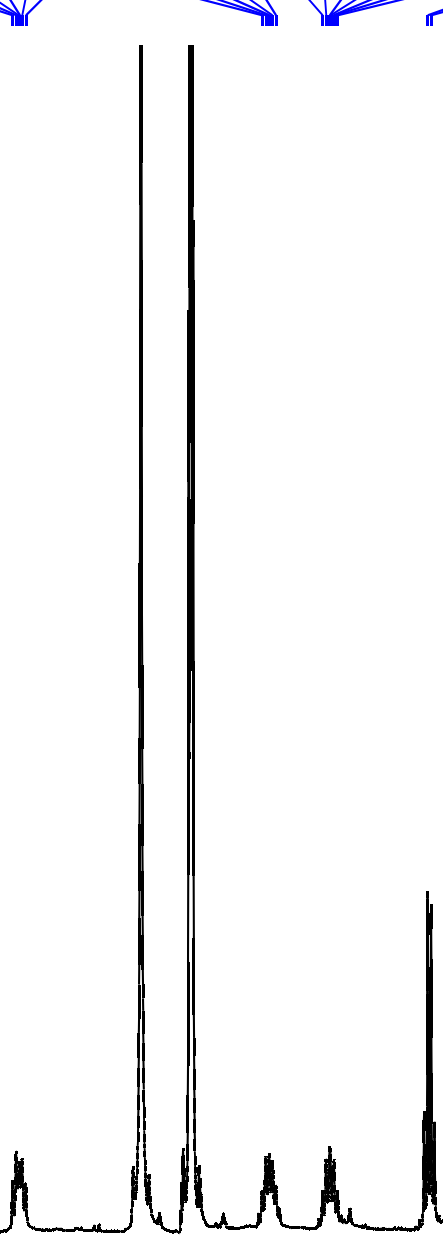


3 - $\{6$ - [5- (4-Bromo-phenyl) - 2H-pyrazol-3-yl] - pyridin-2-yl $\}-5,6$ - bis (4-butyl-phenyl)-[1,2,4]triazine (22) VGB-B-221(32)

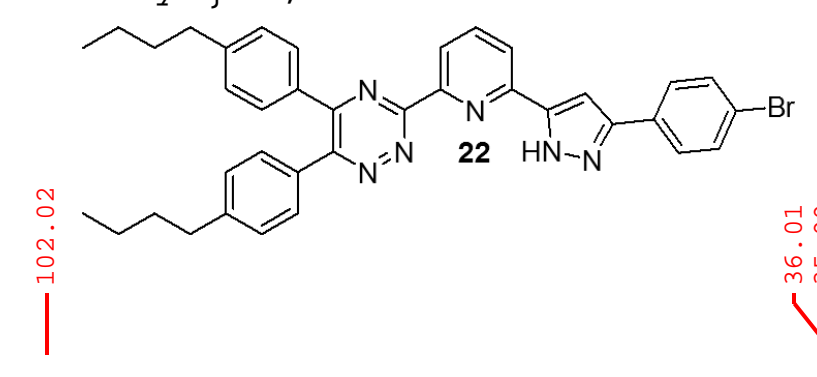

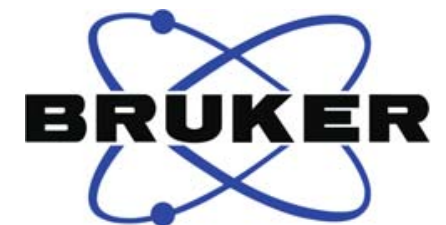

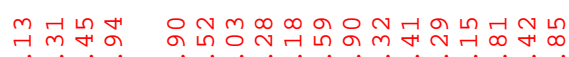

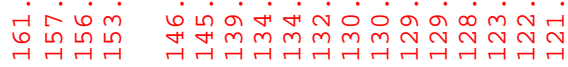

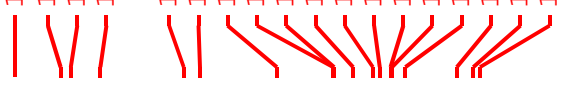

लn

à $\quad$ 舟

กิ $\dot{7} \dot{\exists}$

VI
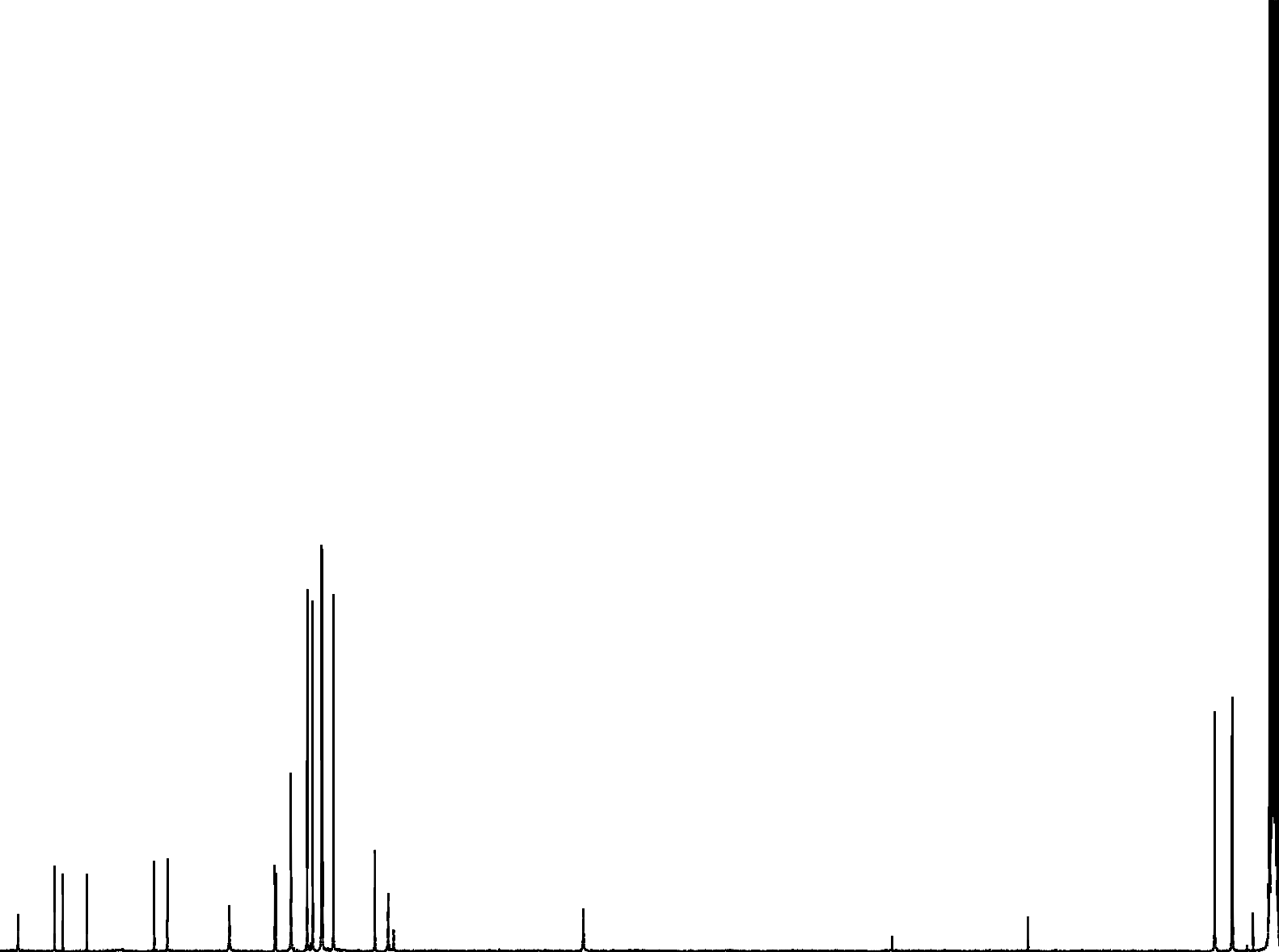

$190 \quad 180-170$

$150 \quad 140$

110100

90

80

70

60

50

40

30

20

10

ppm 
3 - $\{6$ - [5- (4-Bromo - phenyl) - $2 \mathrm{H}$ - pyrazol-3-yl] - pyridin-2-yl $\}-5,6$ - bis [4-(3,3-dimethyl-butyl)-phenyl]-[1,2,4]triazine (23)

VGB-B-99(5)

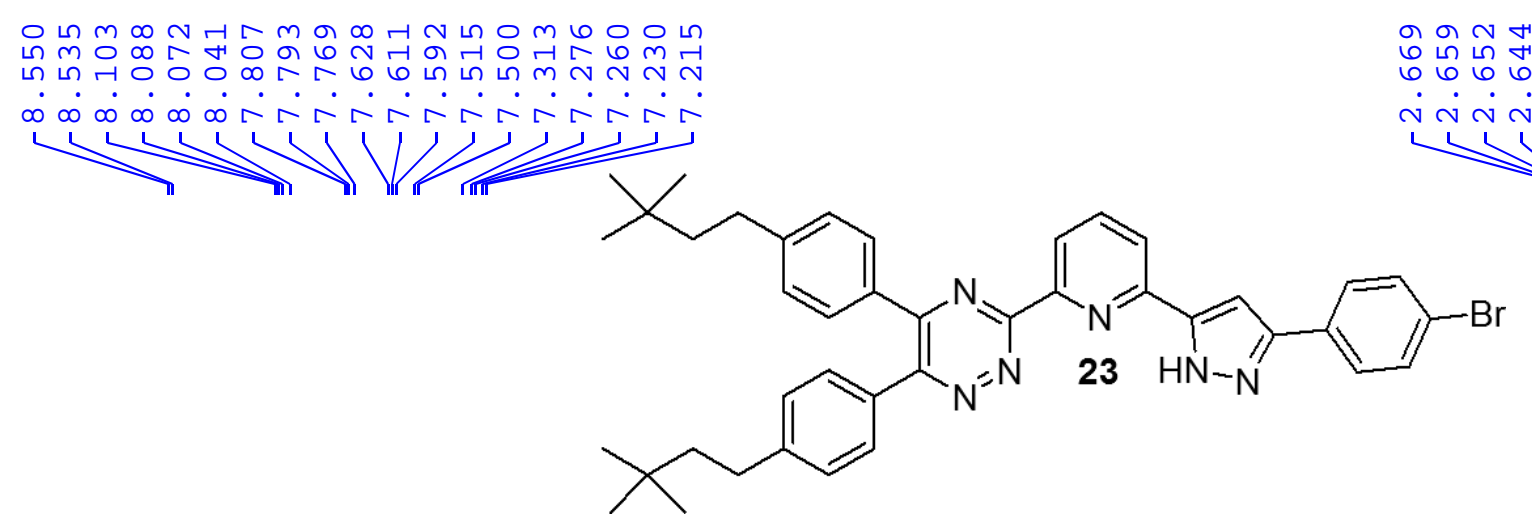

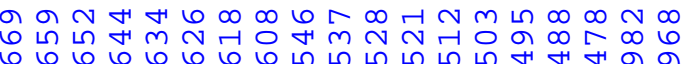
innanañ

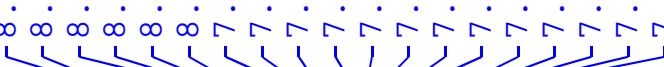

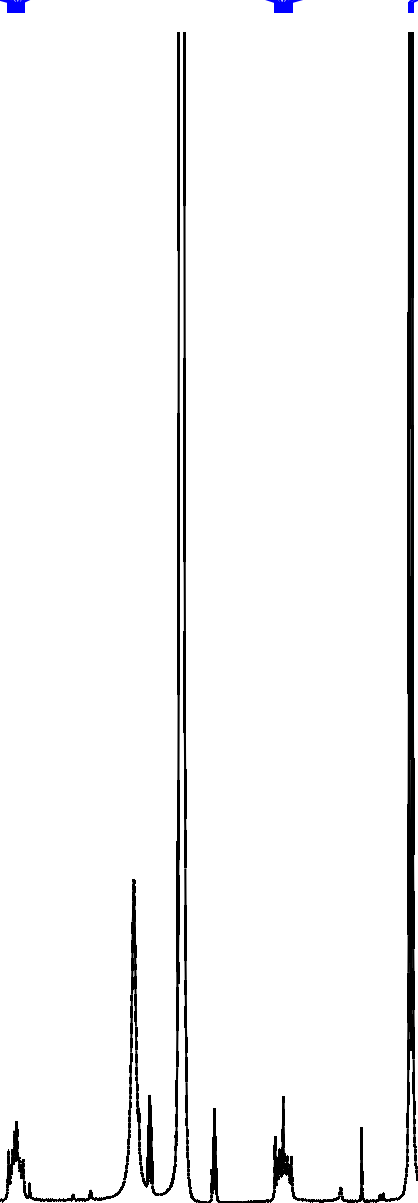

L h l hu

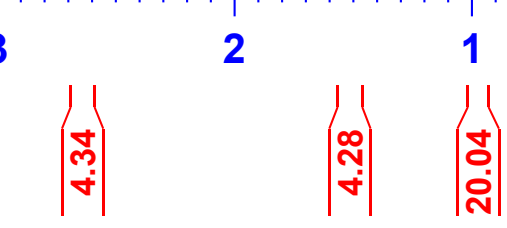


$3-\{6$ - [5- (4-Bromo - phenyl) - 2H-pyrazol - 3-yl] - pyridin-2-yl $\}-5,6$ - bis [4-(3,3-dimethyl-butyl) - phenyl]-[1,2,4]triazine (23) VGB-B-99(5)
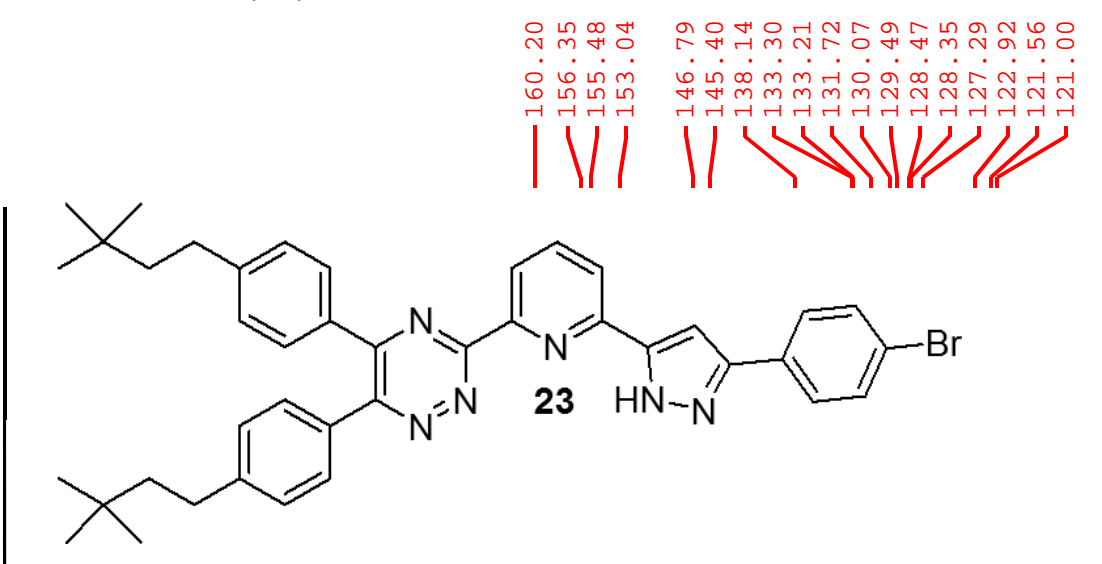

\section{BR UKER}

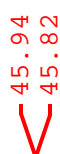

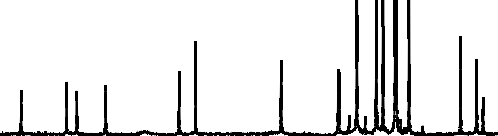


3 - $\{6$ - [5- (4 - tert - Butyl - phenyl) - 2H-pyrazol - 3-yl] - pyridin-2-yl\}5,6 -bis-[4-(3,3-dimethyl-butyl)-phenyl]-[1,2,4] triazine (24) VGB-B-97(5)
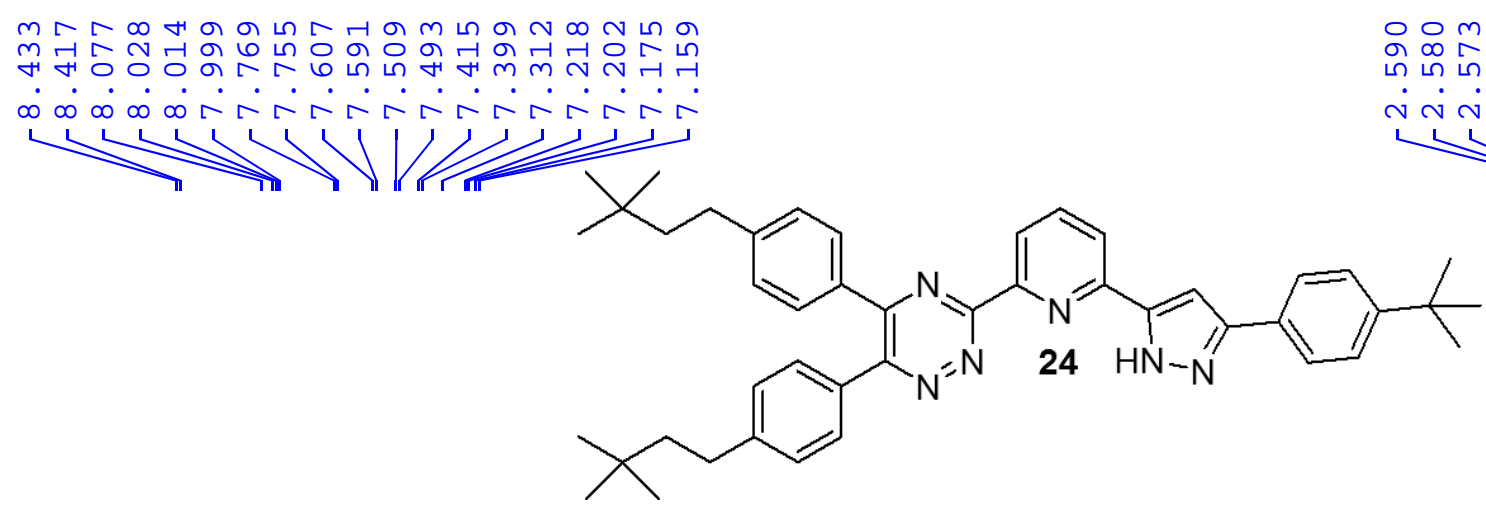

๑ே ஃ

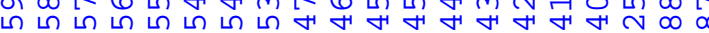

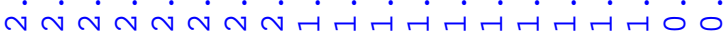

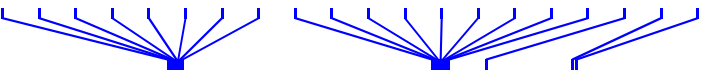

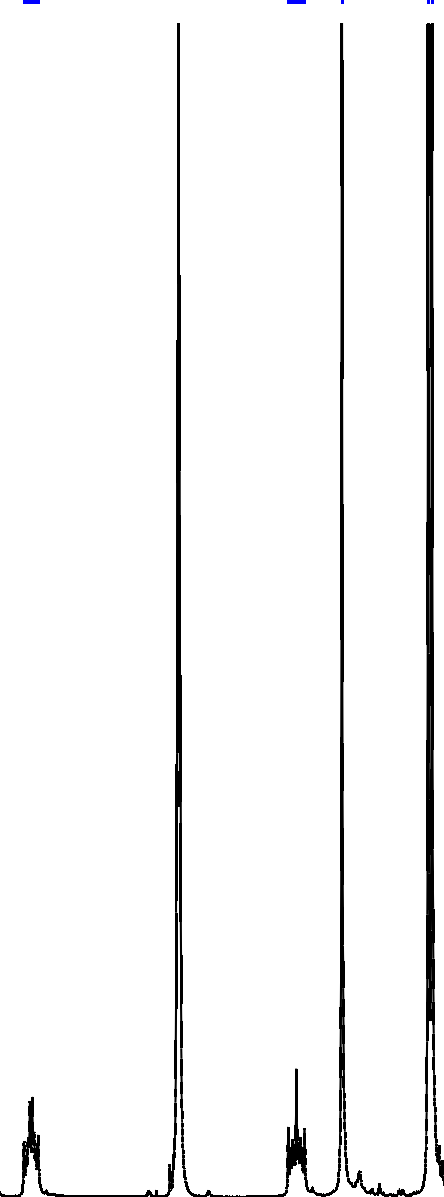

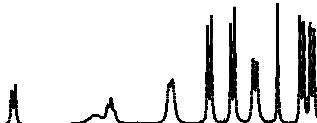

65

5

4

3

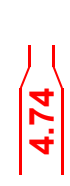

2

1

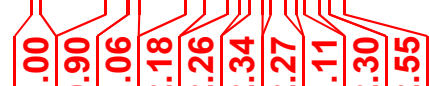

- 
5, 6 -Bis - (4-butyl-phenyl) - 3 - $\{6$ - [5- (3,5-di-tert-butyl-phenyl) - $2 \mathrm{H}$ pyrazol-3-yl]-pyridin-2-yl\}-[1,2,4]triazine (25)

VGB-B-223(5)

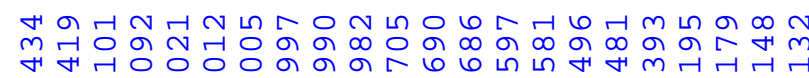

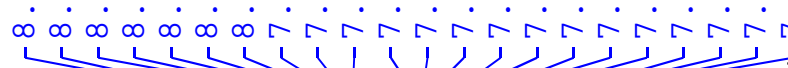

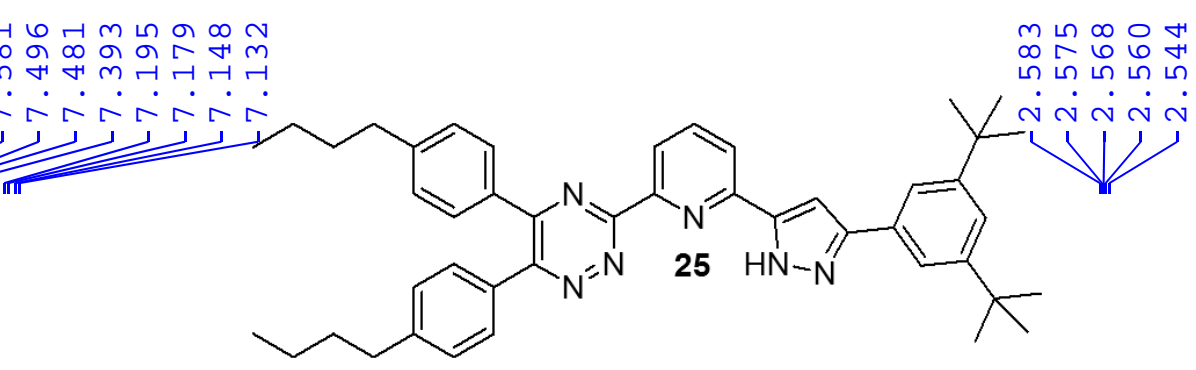

每

-

$\mathrm{W}^{\longrightarrow}$

$\sqrt[1]{4}$
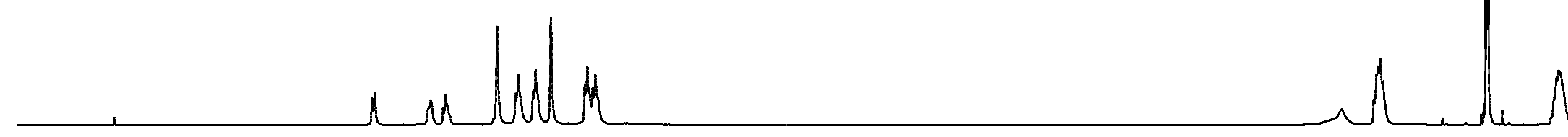

10

8

7

6

5

4

3

$\left|\begin{array}{l}\text { o } \\ \dot{\sigma}\end{array}\right|$

2

1

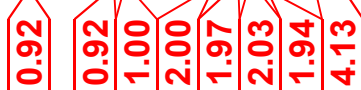


5, 6 -Bis-(4-butyl-phenyl) - $3-\{6$ - [5- (3,5-di-tert-butyl-pheny $\mathrm{l})-2 \mathrm{H}$ pyrazol-3-yl]-pyridin-2-yl\}-[1,2,4]triazine (25) VGB-B-223(5)
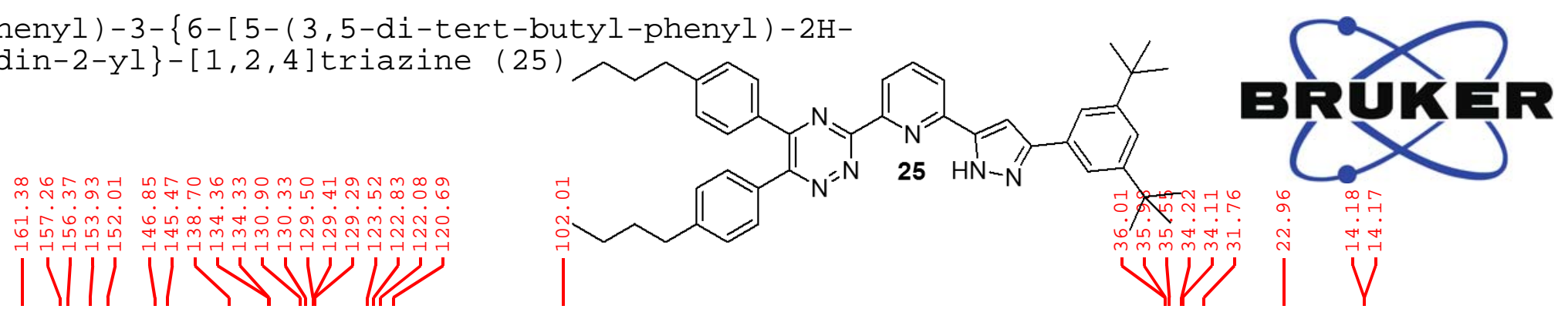

(
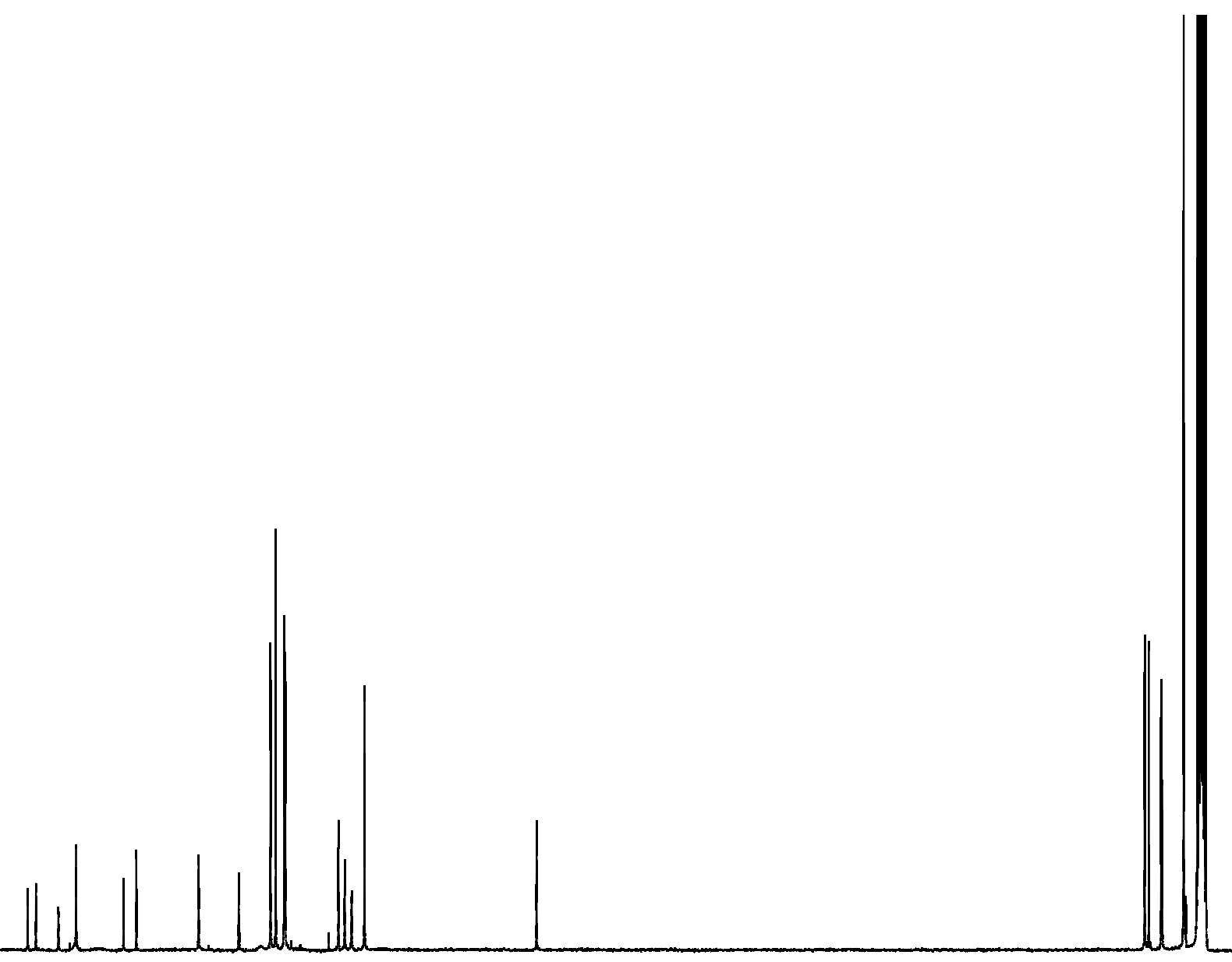

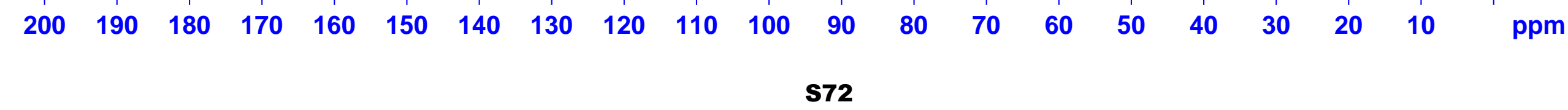


3 - $\{6$ - [5- (4-Bromo - phenyl) - $2 \mathrm{H}-$ pyrazol - 3-yl] - pyridin-2-yl $\}$ 5,6-di-p-tolyl-[1,2,4] triazine (26)

VGB-B-107(5)

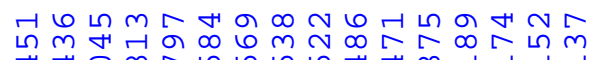

.

$\infty \infty 0$ nNanNanNan
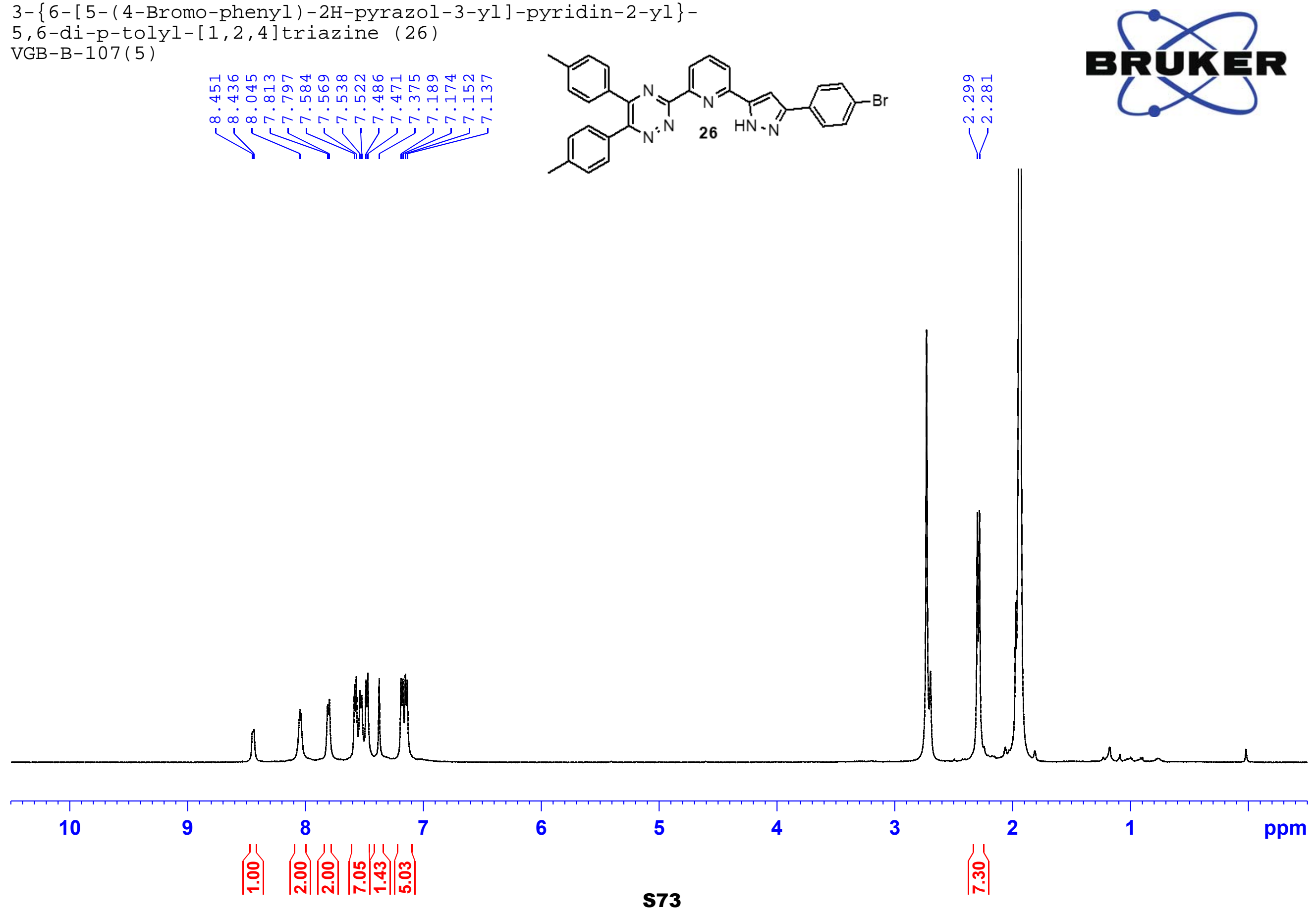


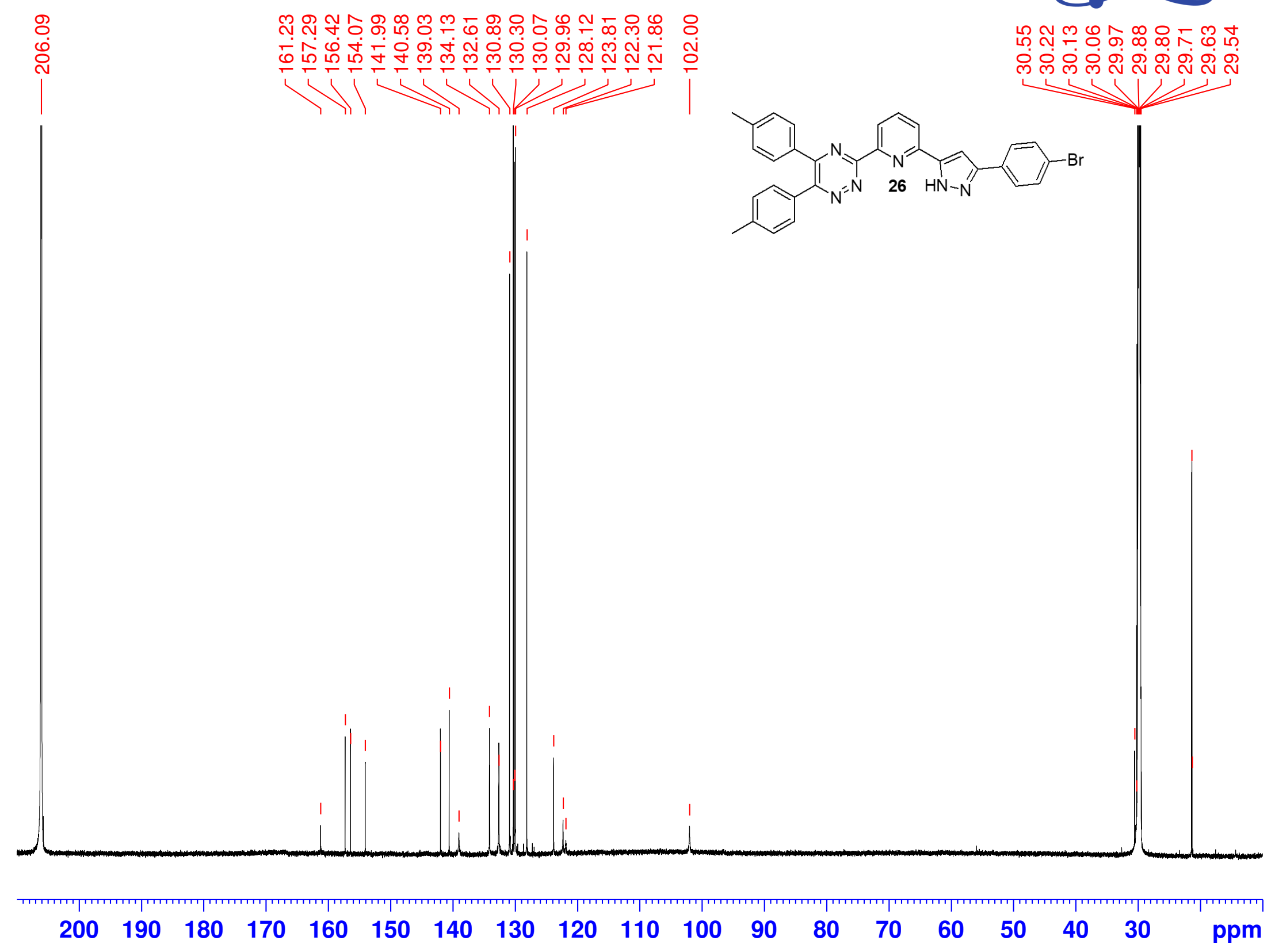


5, 6 -Bis - (4 - fluoro-phenyl) - 3 - $\{6$ - [5 - (4 - methoxy-phenyl) - $2 \mathrm{H}$ pyrazol-3-yl]-pyridin-2-yl\}-[1,2,4]triazine (27)

VGB-B-93(5)

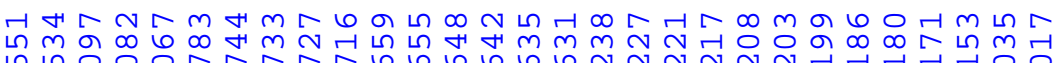

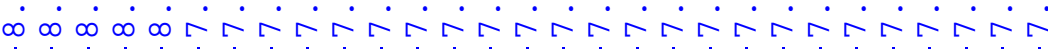

N

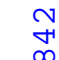

m

A

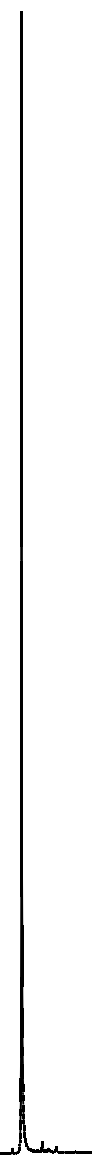

575

\section{BR UKR}

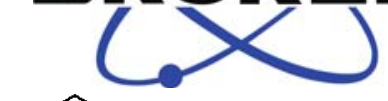

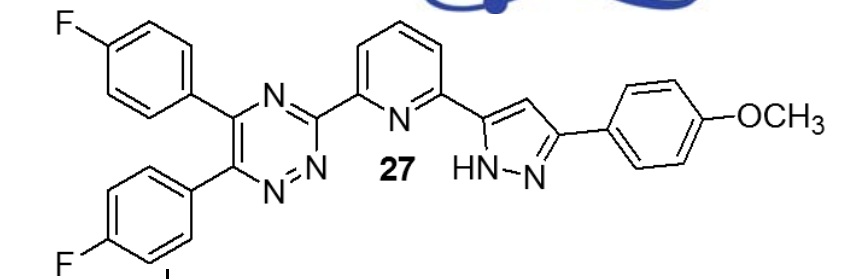

10

9
6

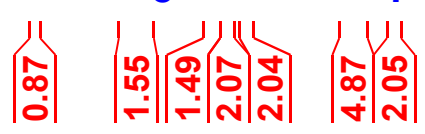

5

$7 \quad 6$

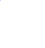

$1+1+1$

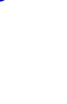

4

$\left|\begin{array}{l}0 \\ 0 \\ 0 \\ j\end{array}\right|$
3

2

1

ppm 


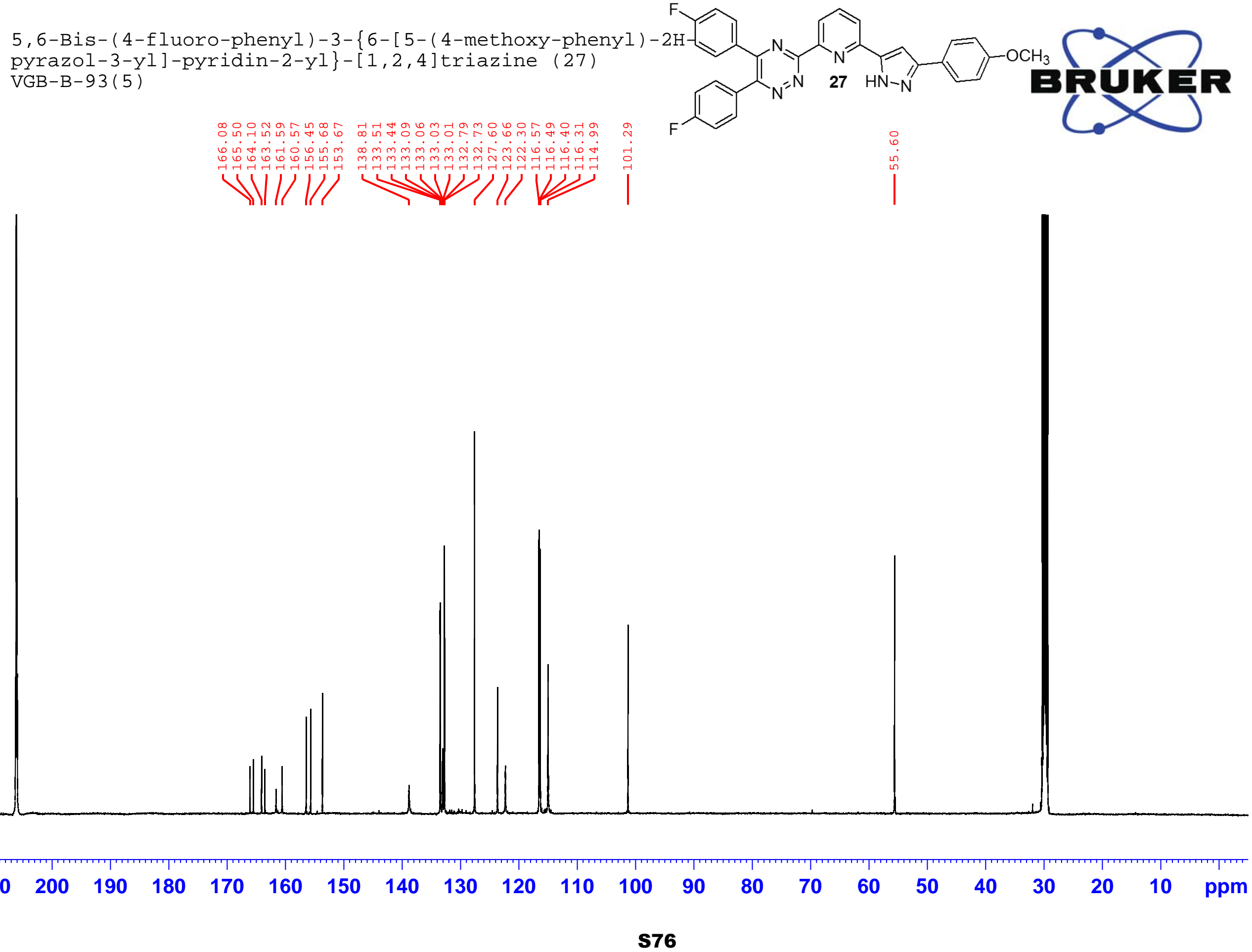


5,6-Bis - (4-cyclopropyl-phenyl) - 3 - $\{6$ - [5- (2, 4, 6-trifluoro-phenyl) - $2 \mathrm{H}$ pyrazol-3-yl]-pyridin-2-yl\}-[1,2,4]triazine (28)
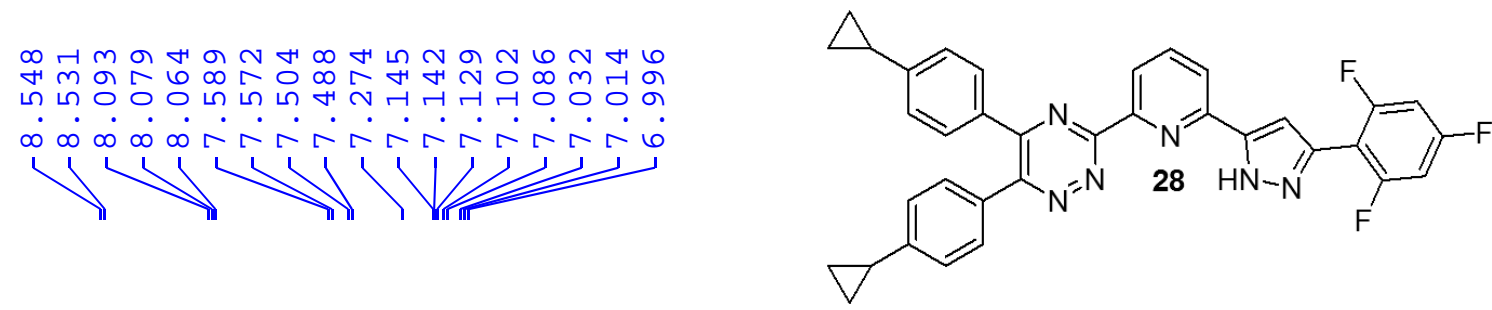

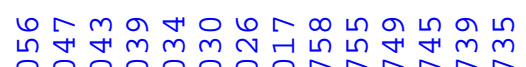

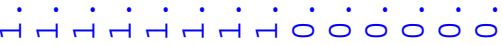
$1<1<1<111$
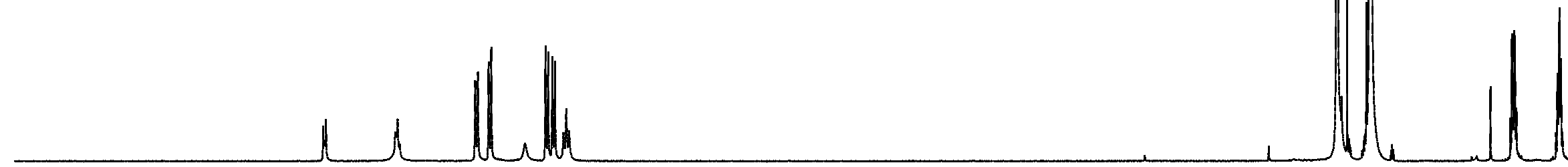

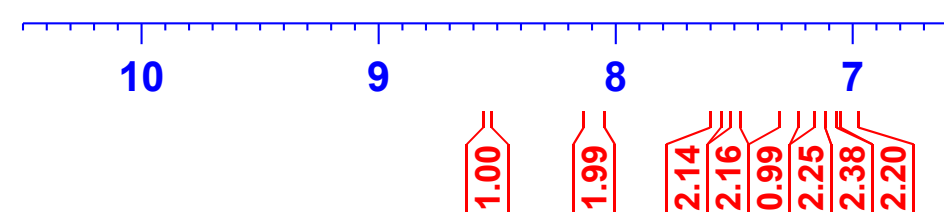

5

S77
3

4

2

1

กิ ppm 
3 - (6-\{5-[4- (4-tert-Butyl-phenylethynyl) - phenyl] - 2H-pyrazol-3-yl $\}$ - pyridin-2-yl) 5,6 -diphenyl-[1,2,4] triazine (29)

VGB-C-57(5)

N

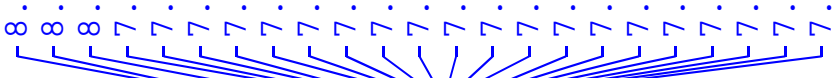

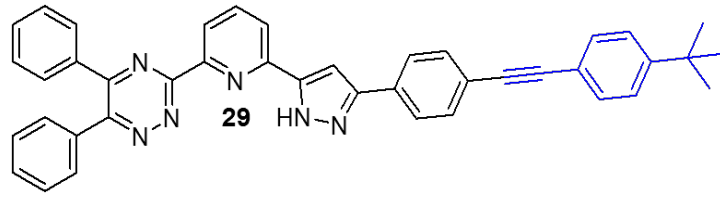

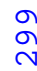

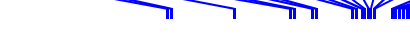
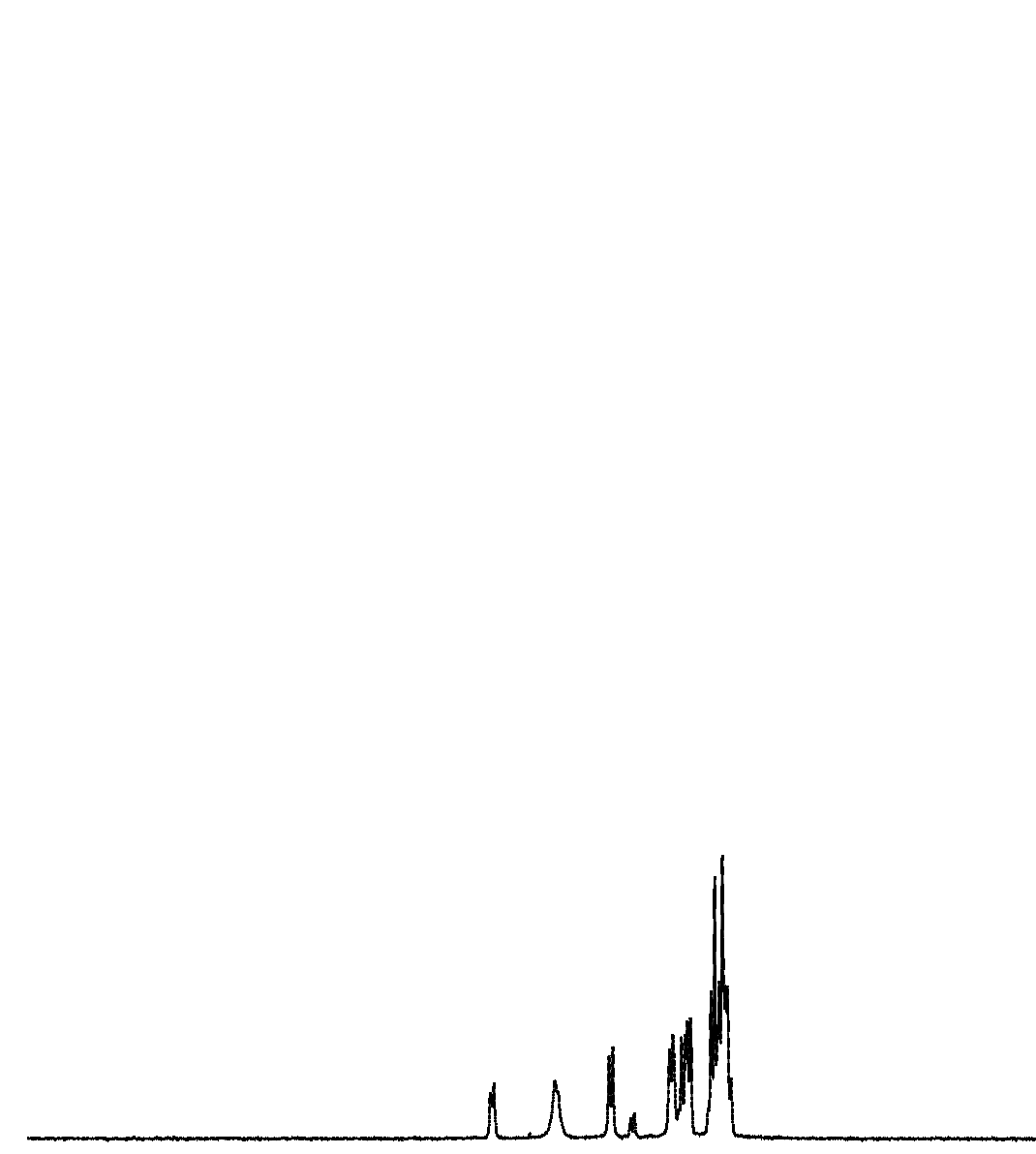

10

9

8

7

6

5

4

3

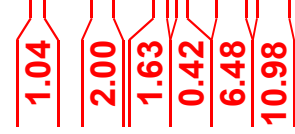

1

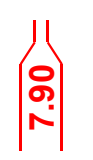

ppm 
3- (6- 65 - [4- (4-tert-Butyl-phenylethynyl) - phenyl] - 2H-pyrazol-3-yl\}pyridin-2-yl)-5,6-diphenyl-[1,2,4] triazine (29)

VGB-B-57(3)
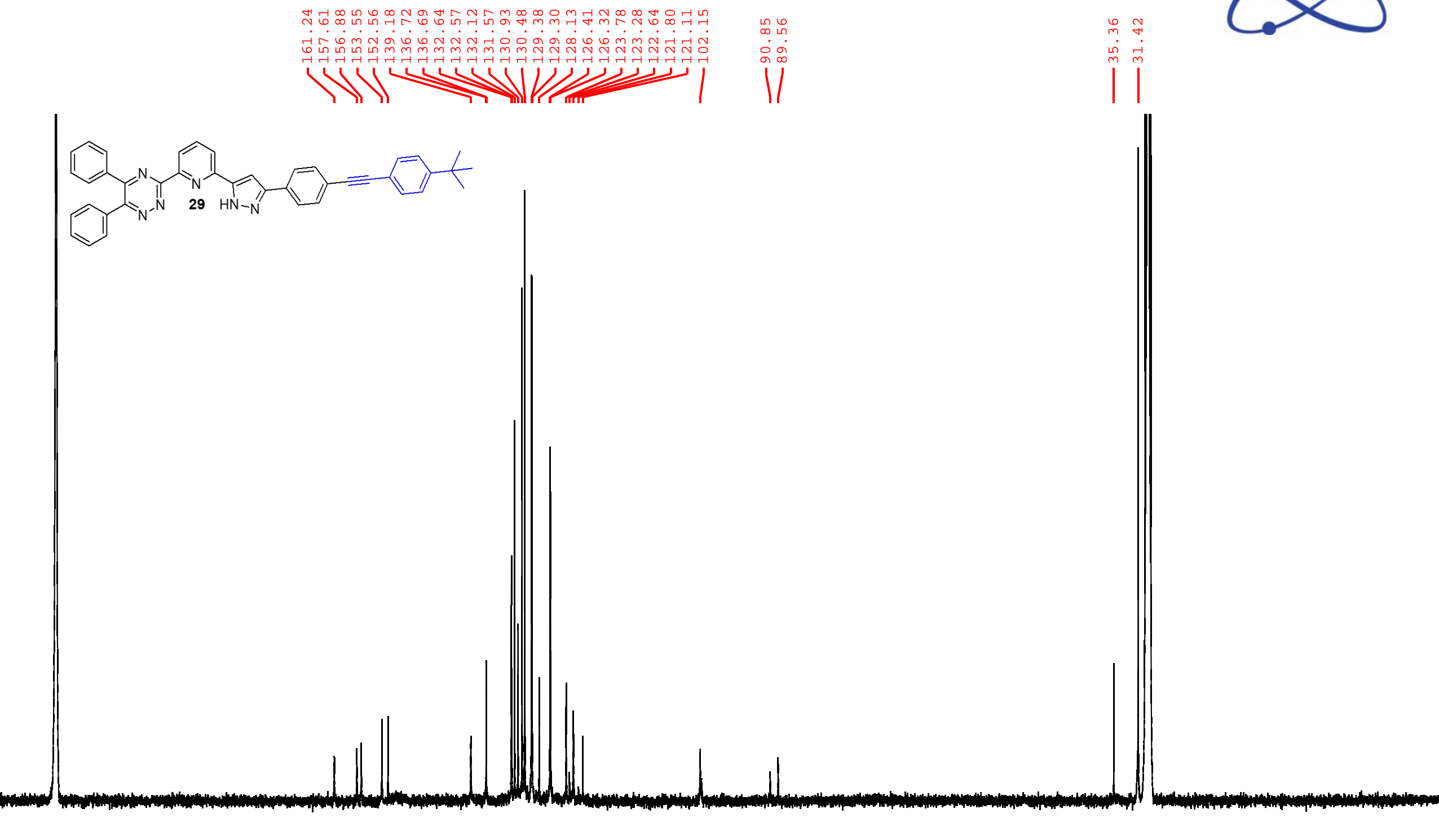
Figure S1. 3-(6-Ethynyl-pyridin-2-yl)-5,6-diphenyl-[1,2,4]triazine (1) Sample: VGB-B-177

4x Column: 2

RediSep Column: Silica 40g

SN: E041501CEEF781 Lot: 232124001Z

Flow Rate: $40 \mathrm{ml} / \mathrm{min}$

Equilibration Volume: $240.0 \mathrm{ml}$

Initial Waste: $0.0 \mathrm{ml}$

Air Purge: $1.0 \mathrm{~min}$

Solvent: A1 hexane

Solvent: B1 ethyl acetate

\section{Rf 200}

Peak Tube Volume: Max.

Non-Peak Tube Volume: Max.

Loading Type: Solid

Wavelength 1 (red): 254nm

Peak Width: 2 min

Threshold: 0.20 AU

Wavelength 2 (purple): $280 \mathrm{~nm}$
Tuesday 18 December 2018 08:18PM

All Wavelength (orange): 200-300nm

Peak Width: 2 min

Threshold: 0.20 AU

Run Notes:

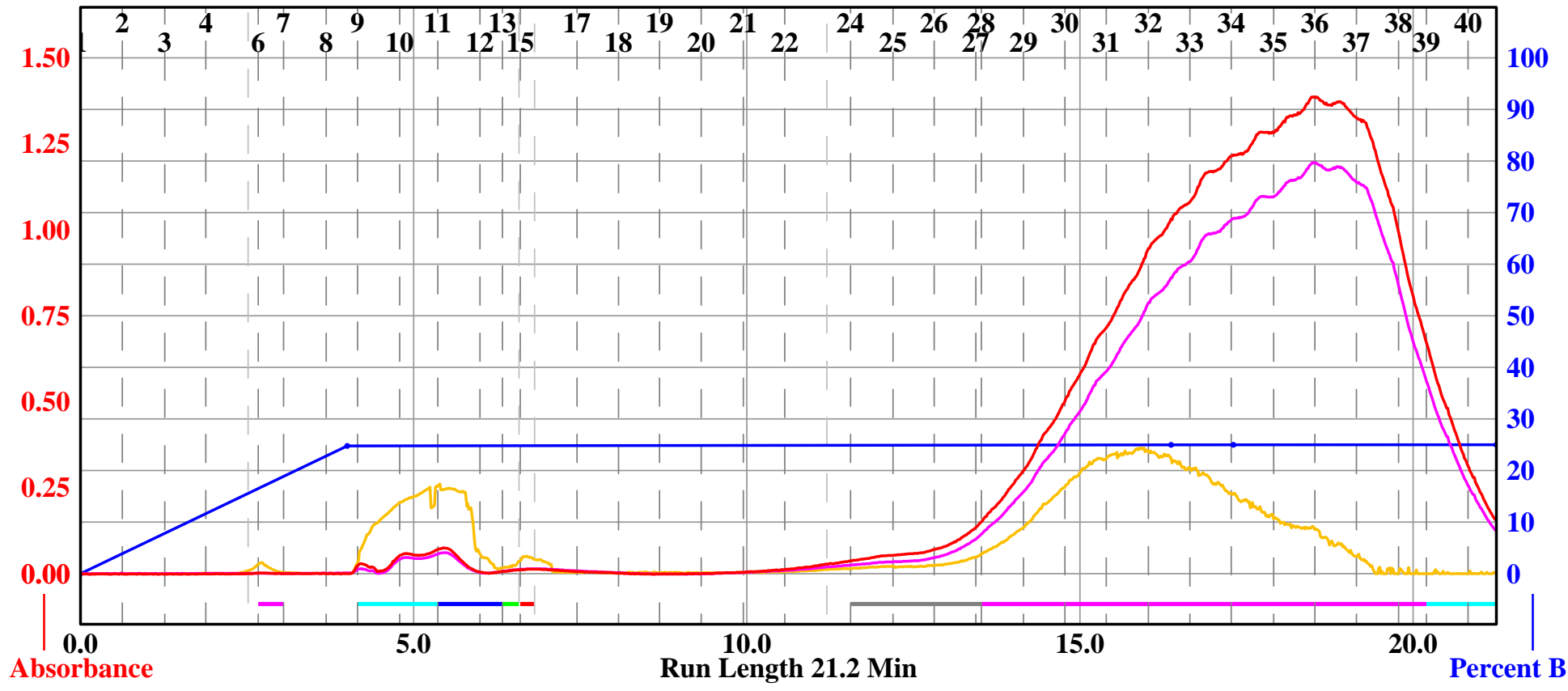

Rack A

\begin{tabular}{|c|c|c|c|c|}
\hline (70) & (69) & (68) & (67) & 66 \\
\hline 1) & (62) & $(63)$ & (64) & 65 \\
\hline D) & (59) & (58) & (57) & (20) \\
\hline (51) & (52) & (53) & (54) & \\
\hline (50) & (49) & (48) & (47) & 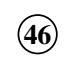 \\
\hline (4) & (42) & (43) & (44) & \\
\hline (40) & (39) & (38) & (37) & \\
\hline & (32) & (33) & (34) & \\
\hline & (29) & (28) & (27) & \\
\hline (2) & (22) & (23) & (24) & $(2$ \\
\hline (20) & (19) & (18) & (17) & \\
\hline & & (13) & (14) & \\
\hline (10) & (9) & (8) & (7) & 6 \\
\hline (1) & (2) & (3) & (4) & \\
\hline
\end{tabular}

Peak \#

Start Tube

A:6

A:9

A:11

A:13

A:15

A:24

A:28

A:39

7

\begin{tabular}{|c|c|c|c|}
\hline \multicolumn{1}{c}{ Duration } & \multicolumn{1}{c}{$\%$ B } & Solvent A & Solvent B \\
\hline $\mathbf{0 . 0}$ & $\mathbf{0 . 0}$ & A1 hexane & B1 ethyl acetate \\
4.0 & $\mathbf{2 4 . 8}$ & A1 hexane & B1 ethyl acetate \\
12.4 & $\mathbf{2 5 . 0}$ & A1 hexane & B1 ethyl acetate \\
0.9 & 25.0 & A1 hexane & B1 ethyl acetate \\
3.9 & $\mathbf{2 5 . 0}$ & A1 hexane & B1 ethyl acetate \\
& & & \\
& & & \\
& & & \\
& & & \\
\hline
\end{tabular}



Figure S2. 3-(6-Ethynyl-pyridin-2-yl)-5,6-di-p-tolyl-[1,2,4]triazine (4)
Sample: VGB-B-103

4x Column: 2

RediSep Column: Silica 12g Gold

SN: E0415093D6276B Lot: 281928401Y

Flow Rate: $30 \mathrm{ml} / \mathrm{min}$

Equilibration Volume: $100.8 \mathrm{ml}$

Initial Waste: $0.0 \mathrm{ml}$

Air Purge: 0.5 min

Solvent: A1 hexane

Solvent: B1 ethyl acetate
Peak Tube Volume: Max.

Non-Peak Tube Volume: Max.

Loading Type: Solid

Wavelength 1 (red): 254nm

Peak Width: 1 min

Threshold: $0.20 \mathrm{AU}$

Wavelength 2 (purple): $280 \mathrm{~nm}$
Monday 26 November 2018 08:54AM

All Wavelength (orange): 200-300nm Peak Width: 1 min

Threshold: 0.20 AU

Run Notes:

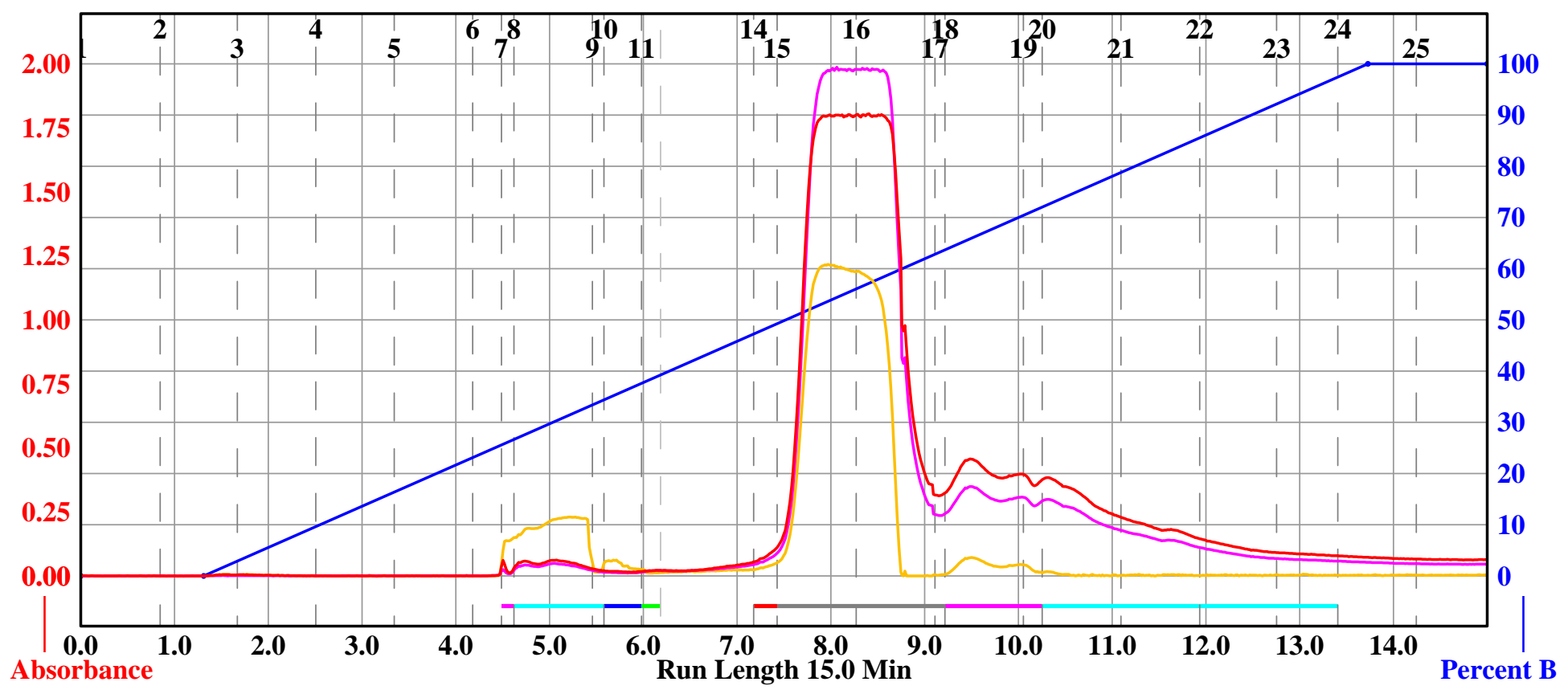

Rack A

\begin{tabular}{|c|c|c|c|c|}
\hline (70) & (69) & (68) & (67) & 66 \\
\hline (61) & (62) & (63) & (64) & 65 \\
\hline (6) & (59) & (58) & (57) & 56 \\
\hline 5 & (52) & (53) & (54) & $\Omega$ \\
\hline $8 \mathrm{P}$ & (49) & (48) & (47) & (46) \\
\hline$(4)$ & (42) & (43) & (44) & 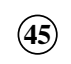 \\
\hline 4 & (39) & (38) & (37) & 36 \\
\hline & (32) & (33) & (34) & (30) \\
\hline (30) & (29) & (28) & (27) & 26 \\
\hline 2 & (22) & (23) & (24) & 2 \\
\hline 24 & (19) & (18) & (17) & (16) \\
\hline$(11)$ & (12) & (13) & (14) & 1. \\
\hline & (9) & (8) & (7) & 6 \\
\hline & (2) & (3) & (4) & 5 \\
\hline
\end{tabular}

Peak \#

Start Tube

A:7

A:8

A:10

A:11

A:14

A:15

A:18

A:20

End Tube

A:7

A:9

A:10

A:11

A:14

A:17

A:19

A:23

\begin{tabular}{|c|c|c|c|}
\hline \multicolumn{1}{|c}{ Duration } & \multicolumn{1}{c}{$\%$ B } & Solvent A & Solvent B \\
\hline $\mathbf{0 . 0}$ & $\mathbf{0 . 0}$ & A1 hexane & B1 ethyl acetate \\
1.3 & $\mathbf{0 . 0}$ & A1 hexane & B1 ethyl acetate \\
12.4 & $\mathbf{1 0 0 . 0}$ & A1 hexane & B1 ethyl acetate \\
1.3 & $\mathbf{1 0 0 . 0}$ & A1 hexane & B1 ethyl acetate \\
& & & \\
& & & \\
& & & \\
& & & \\
& & & \\
\hline
\end{tabular}


Figure S3. 5,6-Bis-(4-butyl-phenyl)-3-(6-ethynyl-pyridin-2-yl)-[1,2,4] triazine (5)
Sample: VGB-B.-161
Thursday

4x Column: 2

RediSep Column: Silica 12g

SN: E041508C98E45C Lot: 281639102W

Flow Rate: $30 \mathrm{ml} / \mathrm{min}$

Equilibration Volume: $100.8 \mathrm{ml}$

Initial Waste: $0.0 \mathrm{ml}$

Air Purge: 0.5 min

Solvent: A1 hexane

Solvent: B1 ethyl acetate
Peak Tube Volume: Max.

Non-Peak Tube Volume: Max.

Loading Type: Solid

Wavelength 1 (red): 254nm

Peak Width: 1 min

Threshold: 0.20 AU

Wavelength 2 (purple): 280nm
Thursday 13 December 2018 02:30PM

All Wavelength (orange): 200-300nm

Peak Width: 1 min

Threshold: 0.20 AU

Run Notes:

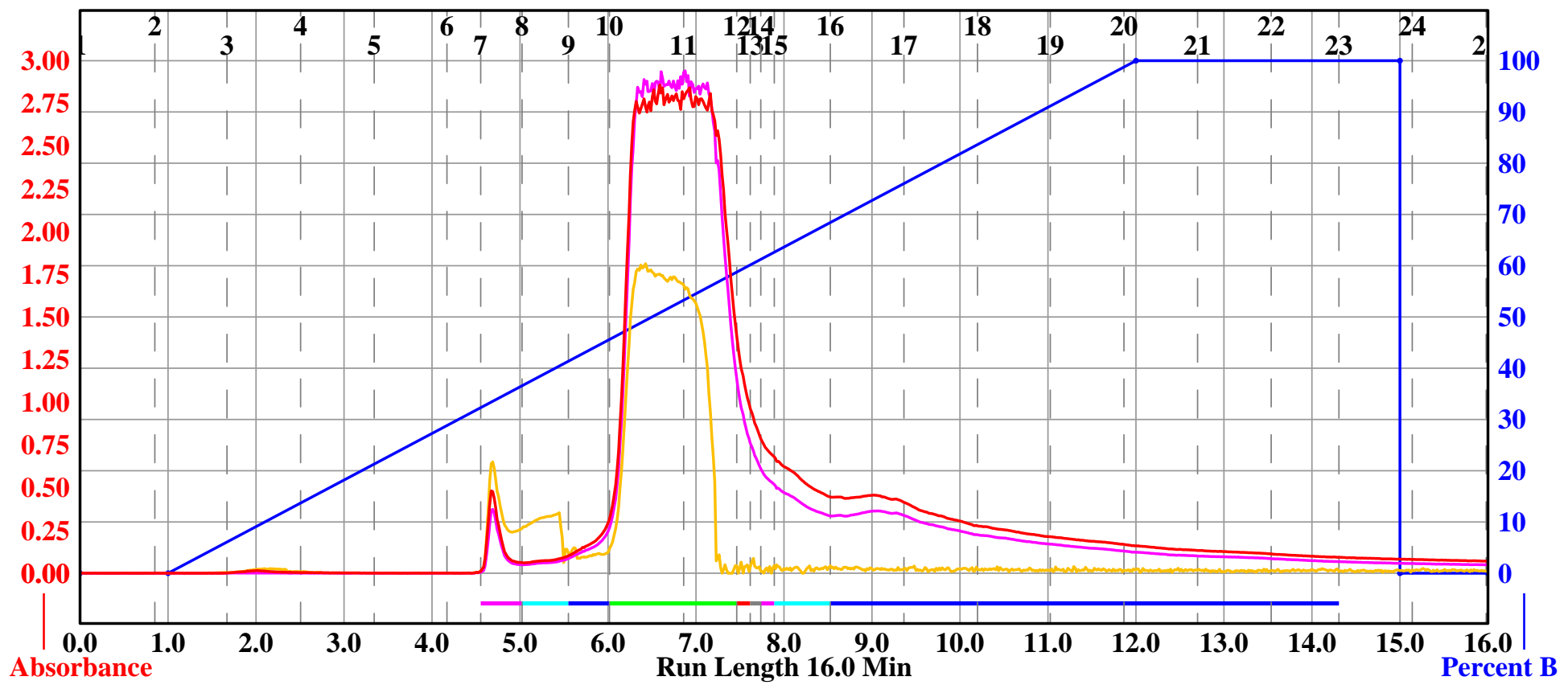

Rack A

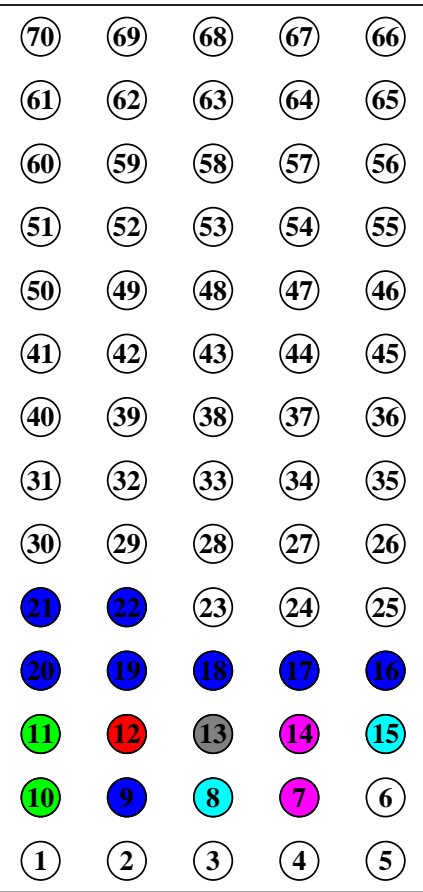

$18 \mathrm{~mm} \times 150 \mathrm{~mm}$ Tubes

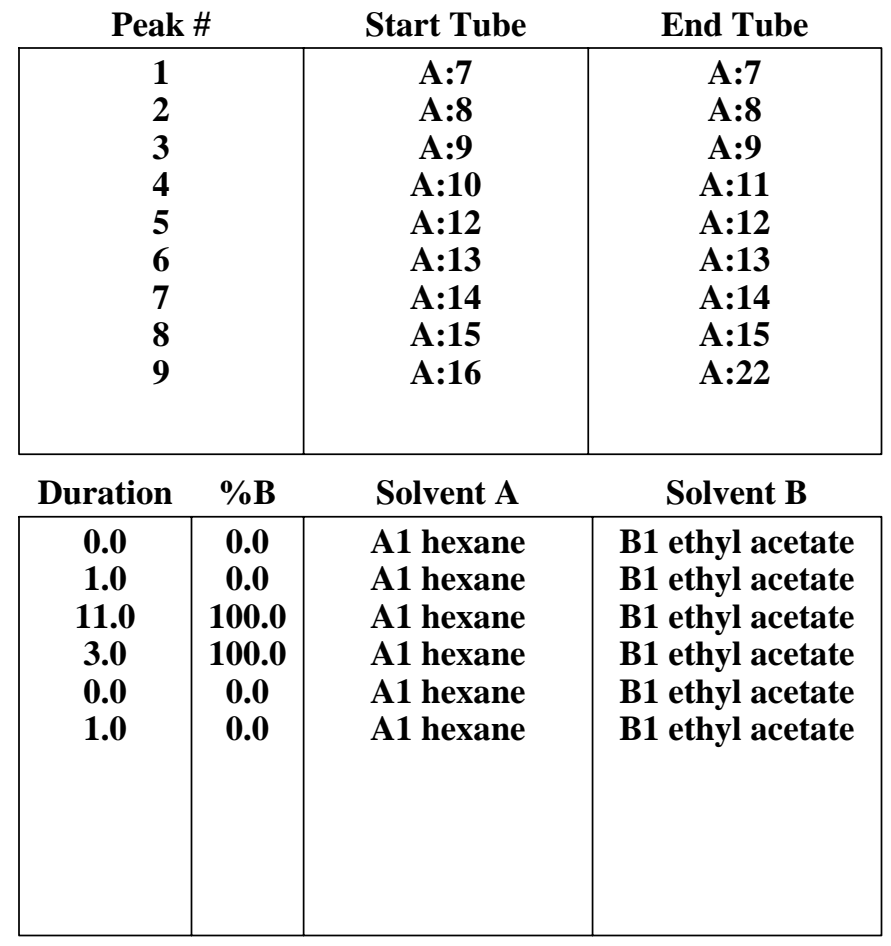


Figure S4 3-(6-Ethynyl-pyridin-2-yl)-5,6-bis-(4-fluoro-phenyl)-[1,2,4] triazine (6)

Sample: VGB-B-83-1

4x Column: 2

RediSep Column: Silica 12g Gold

SN: E0415093D62C38 Lot: 281928401Y

Flow Rate: $30 \mathrm{ml} / \mathrm{min}$

Equilibration Volume: $100.8 \mathrm{ml}$

Initial Waste: $0.0 \mathrm{ml}$

Air Purge: 0.5 min

Solvent: A1 hexane

Solvent: B1 ethyl acetate
Peak Tube Volume: Max.

Non-Peak Tube Volume: Max.

Loading Type: Solid

Wavelength 1 (red): 254nm

Peak Width: 1 min

Threshold: 0.20 AU

Wavelength 2 (purple): 280nm

Tuesday 20 November 2018 04:46PM

All Wavelength (orange): 200-300nm

Peak Width: 1 min

Threshold: 0.20 AU

Run Notes:

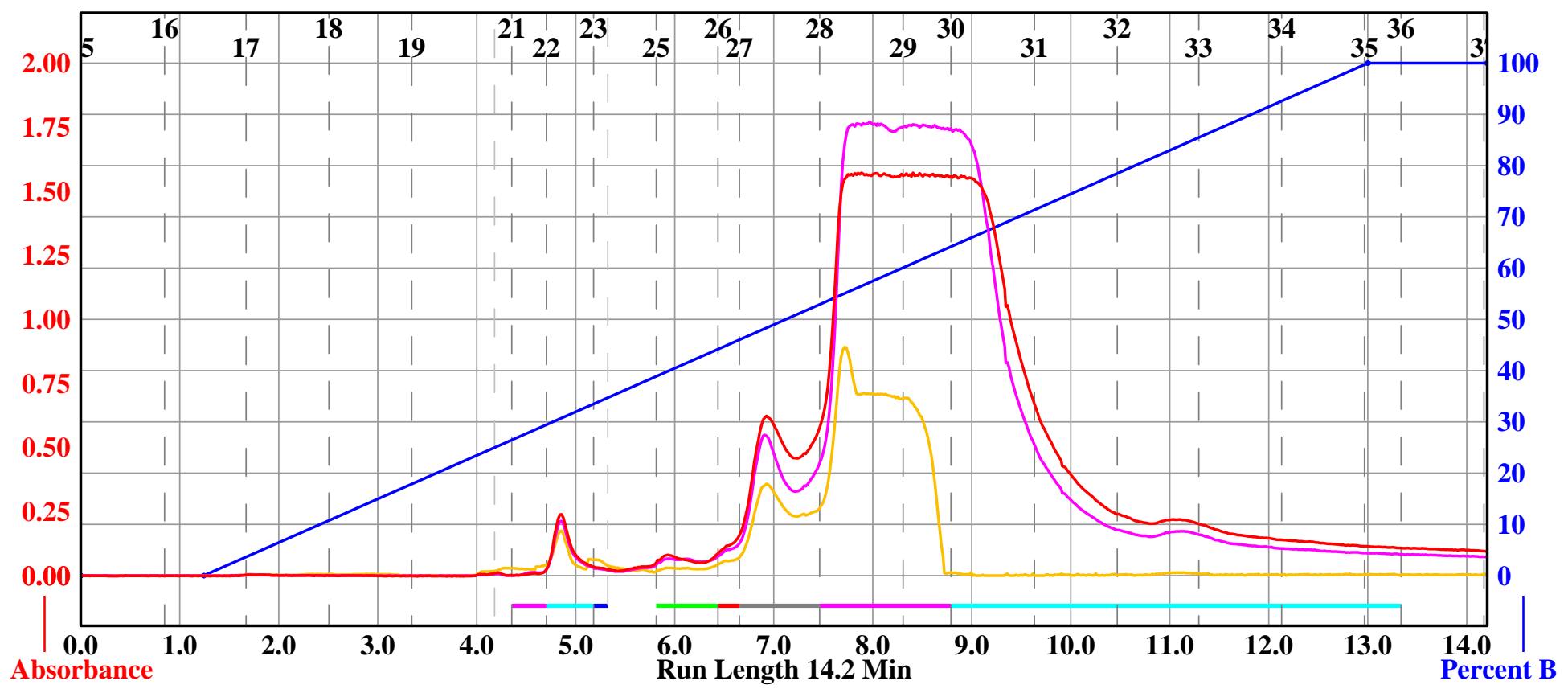

Rack A

\begin{tabular}{|c|c|c|c|c|}
\hline (70) & (69) & (68) & (67) & 66 \\
\hline 61 & (62) & (63) & (64) & (1) \\
\hline 60 & (59) & (58) & (57) & 56 \\
\hline (51) & (52) & (53) & (54) & \\
\hline 50 & (49) & (48) & (47) & (46) \\
\hline 4 & (42) & (43) & (44) & 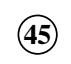 \\
\hline (40) & (39) & (38) & (37) & 36 \\
\hline (31) & (32) & (33) & (34) & (2) \\
\hline (30) & (29) & (2) & (27) & $\sigma$ \\
\hline & (22) & & (24) & (25) \\
\hline (20) & (19) & (18) & (17) & 16 \\
\hline (11) & (12) & (13) & (14) & (1. \\
\hline 11 & (9) & (8) & (7) & 6 \\
\hline$\curvearrowright$ & (2) & (3) & (4) & 6 \\
\hline
\end{tabular}

$18 \mathrm{~mm} \times 150 \mathrm{~mm}$ Tubes

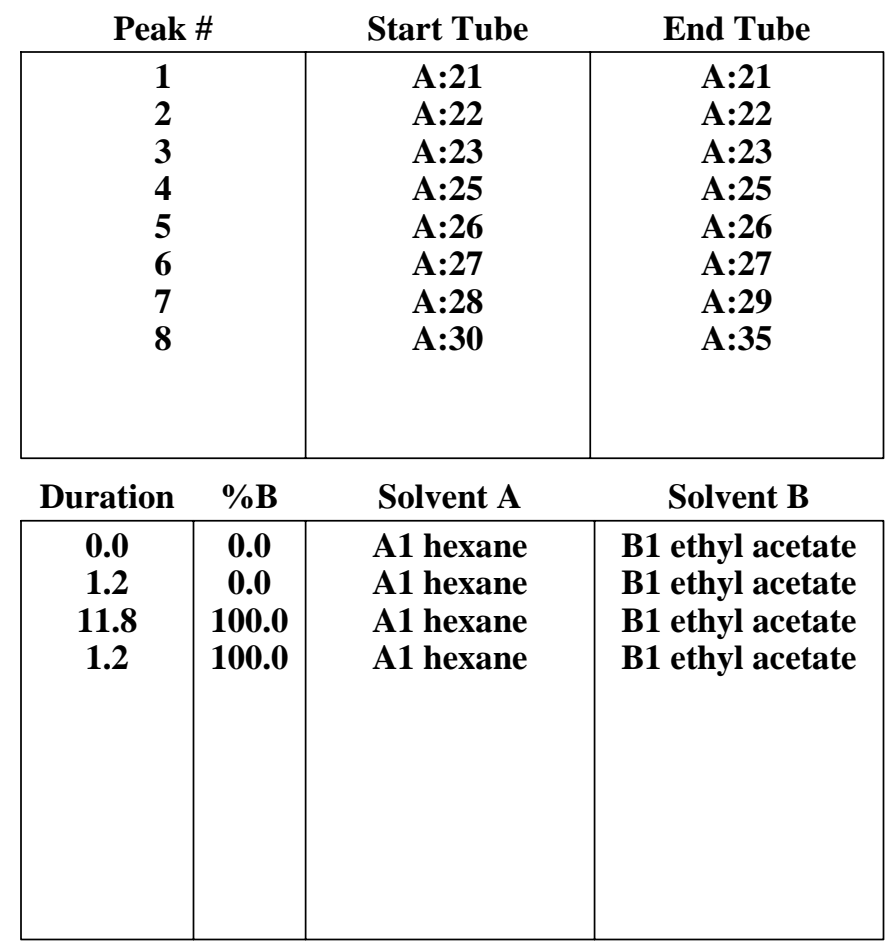




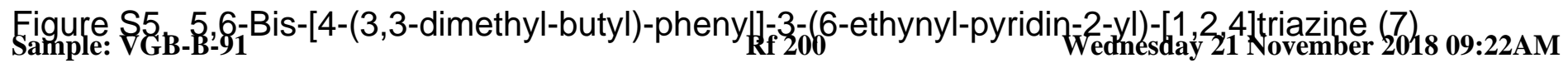

4x Column: 2

RediSep Column: Silica 12g Gold

SN: E0415093D63242 Lot: $281928401 Y$

Flow Rate: $30 \mathrm{ml} / \mathrm{min}$

Equilibration Volume: $100.8 \mathrm{ml}$

Initial Waste: $0.0 \mathrm{ml}$

Air Purge: 0.5 min

Solvent: A1 hexane

Solvent: B1 ethyl acetate
Peak Tube Volume: Max.

Non-Peak Tube Volume: Max.

Loading Type: Solid

Wavelength 1 (red): 254nm

Peak Width: 1 min

Threshold: 0.20 AU

Wavelength 2 (purple): 280nm
All Wavelength (orange): $200-300 \mathrm{~nm}$

Peak Width: 1 min

Threshold: 0.20 AU

Run Notes:

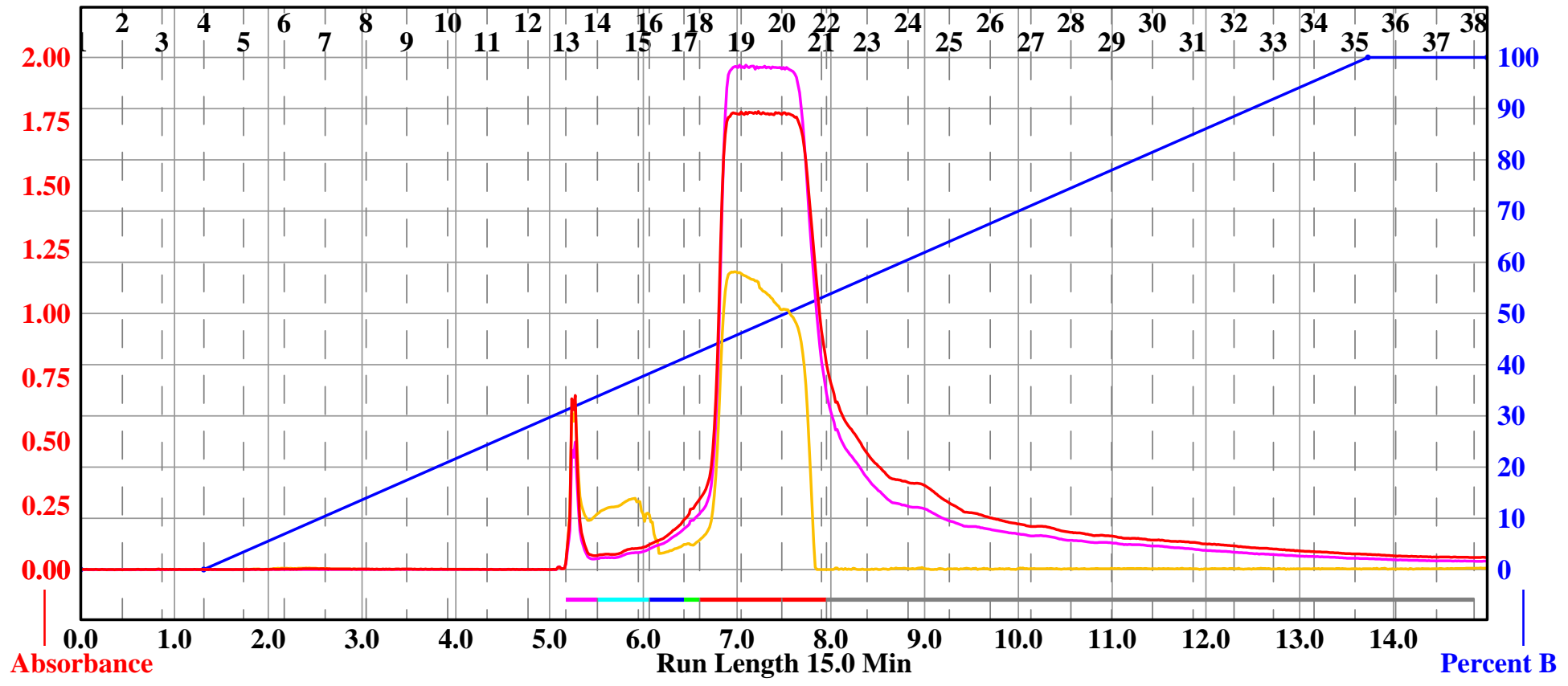

Rack A

\begin{tabular}{|c|c|c|c|}
\hline (71) & (72) & (13) & (14) \\
\hline (70) & (69) & (68) & (67) \\
\hline (61) & (62) & (63) & (64) \\
\hline (60) & (59) & (58) & (57) \\
\hline (51) & (52) & (53) & (54) \\
\hline (50) & (49) & (48) & (47) \\
\hline (41) & (42) & (43) & (44) \\
\hline (40) & (39) & (38) & (37) \\
\hline II) & (32) & (33) & (34) \\
\hline (30) & (29) & (28) & (27) \\
\hline (21) & (22) & (23) & (24) \\
\hline (20) & (19) & (18) & (17) \\
\hline (11) & (12) & (13) & (14) \\
\hline (10) & (9) & (8) & (7) \\
\hline (1) & (2) & (3) & (4) \\
\hline
\end{tabular}

$16 \mathrm{~mm} \times 100 \mathrm{~mm}$ Tubes

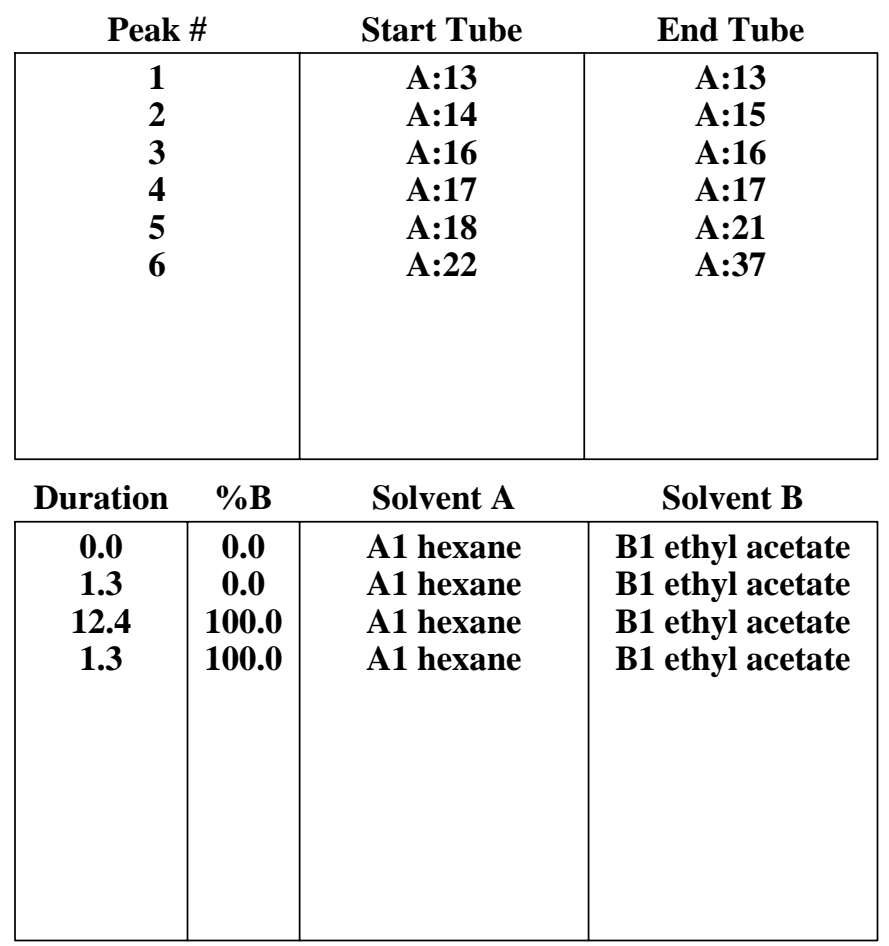



Figure S6.
Sample: VGB-B-263
56-Bis-(4-cyclopropyl-phenyl)-3-(6-ethynyl-pyridin-2-yl)-[1,2,4]triazine
$\mathbf{R f}$ 200

4x Column: 2

RediSep Column: Silica $24 \mathrm{~g}$

SN: E041508C98A11 Lot: 282217407X

Flow Rate: $35 \mathrm{ml} / \mathrm{min}$

Equilibration Volume: $168.0 \mathrm{ml}$

Initial Waste: $0.0 \mathrm{ml}$

Air Purge: $1.0 \mathrm{~min}$

Solvent: A1 hexane

Solvent: B1 ethyl acetate
Peak Tube Volume: Max.

Non-Peak Tube Volume: Max.

Loading Type: Solid

Wavelength 1 (red): 254nm

Peak Width: 1 min

Threshold: $0.20 \mathrm{AU}$

Wavelength 2 (purple): $280 \mathrm{~nm}$

Thursday 24 January 2019 07:56PM

All Wavelength (orange): 200-300nm

Peak Width: 1 min

Threshold: 0.20 AU

Run Notes:

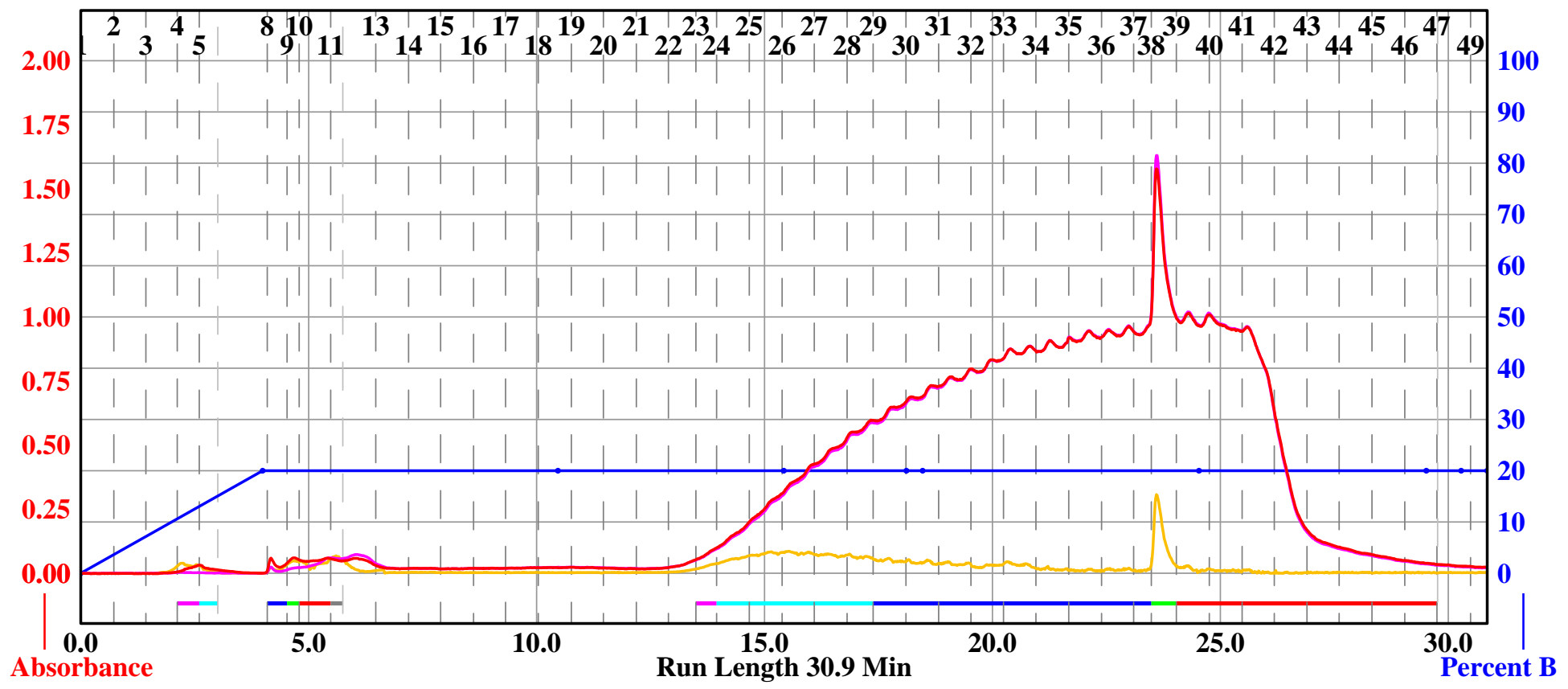

Rack A

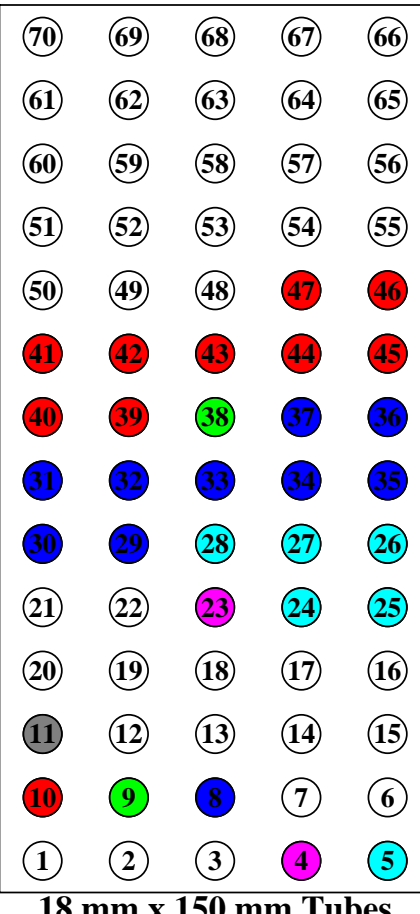

\begin{tabular}{|c|c|c|c|}
\hline \multicolumn{2}{|c|}{ Peak \# } & Start Tube & End Tube \\
\hline \multicolumn{2}{|l|}{1} & A:4 & A:4 \\
\hline \multicolumn{2}{|l|}{2} & A:5 & A:5 \\
\hline \multicolumn{2}{|l|}{3} & A:8 & A:8 \\
\hline \multicolumn{2}{|l|}{4} & A:9 & A:9 \\
\hline \multicolumn{2}{|l|}{5} & A:10 & A:10 \\
\hline \multicolumn{2}{|l|}{6} & A:11 & A:11 \\
\hline \multicolumn{2}{|l|}{7} & $A: 23$ & $A: 23$ \\
\hline \multicolumn{2}{|l|}{8} & A:24 & $A: 28$ \\
\hline \multicolumn{2}{|l|}{9} & $A: 29$ & $A: 37$ \\
\hline$\cdots$ & & $\cdots$ & $\cdots$ \\
\hline Duration & $\%$ B & Solvent A & Solvent B \\
\hline 0.0 & 0.0 & A1 hexane & B1 ethyl acetate \\
\hline 4.0 & 20.0 & A1 hexane & B1 ethyl acetate \\
\hline 6.5 & 20.0 & A1 hexane & B1 ethyl acetate \\
\hline 5.0 & 20.0 & A1 hexane & B1 ethyl acetate \\
\hline 2.7 & 20.0 & A1 hexane & B1 ethyl acetate \\
\hline 0.4 & 20.0 & A1 hexane & B1 ethyl acetate \\
\hline 6.1 & 20.0 & A1 hexane & B1 ethyl acetate \\
\hline 5.0 & 20.0 & A1 hexane & B1 ethyl acetate \\
\hline 0.8 & 20.0 & A1 hexane & B1 ethyl acetate \\
\hline 0.6 & 20.0 & A1 hexane & B1 ethyl acetate \\
\hline
\end{tabular}


Figure S7. 5,6-Diphenyl-3-[6-(5-phenyl-2H-pyrazol-3-yl)-pyridin-2-yl]-[1, 2,4]triazine (9)
Sample: VGB-B-19 200 (hursday 08 November 2018 11:06AM

4x Column: 3

RediSep Column: Silica 4g

Flow Rate: $18 \mathrm{ml} / \mathrm{min}$

Equilibration Volume: $33.6 \mathrm{ml}$

Initial Waste: $0.0 \mathrm{ml}$

Air Purge: 0.5 min

Solvent: A2 dichloromethane

Solvent: B2 2-propanol
Peak Tube Volume: Max.

Non-Peak Tube Volume: Max.

Loading Type: Solid

Wavelength 1 (red): 254nm

Peak Width: 30 sec

Threshold: 0.20 AU

Wavelength 2 (purple): 280nm
All Wavelength (orange): 200-300nm

Peak Width: 30 sec

Threshold: 0.20 AU

Run Notes:

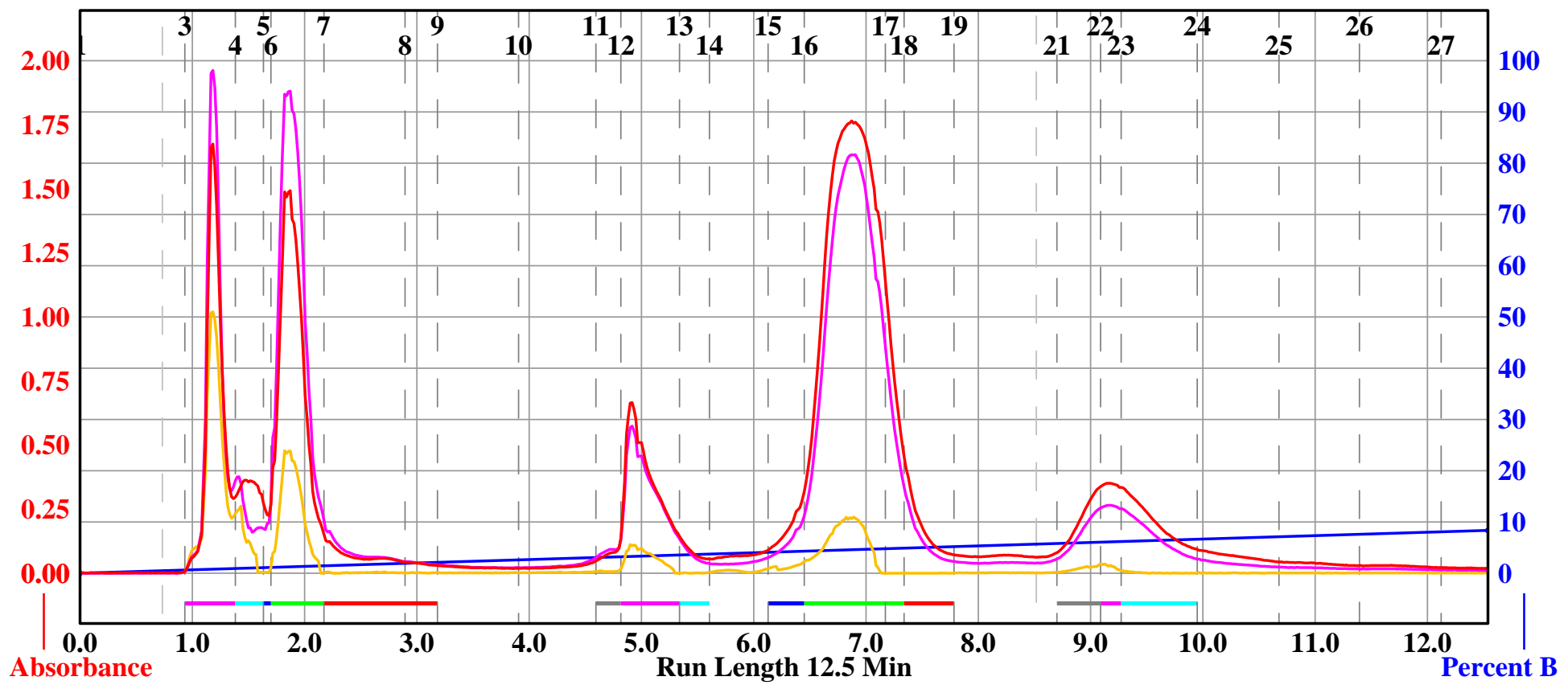

Rack A

\begin{tabular}{|c|c|c|c|}
\hline (71) & (72) & (13) & (14) \\
\hline (70) & (69) & (68) & (67) \\
\hline (61) & (62) & (63) & (64) \\
\hline (60) & (59) & (58) & (57) \\
\hline (51) & (52) & (53) & (54) \\
\hline (50) & (49) & (48) & (47) \\
\hline (41) & (42) & (43) & (44) \\
\hline (40) & (39) & (38) & (37) \\
\hline (31) & (32) & (33) & (34) \\
\hline (30) & (29) & (28) & (27) \\
\hline (21) & (22) & (23) & (24) \\
\hline (20) & (19) & (18) & (17) \\
\hline (11) & (12) & (13) & (14) \\
\hline (10) & (9) & (8) & (7) \\
\hline (1) & (2) & (3) & (4) \\
\hline
\end{tabular}

$16 \mathrm{~mm} \times 100 \mathrm{~mm}$ Tubes

Peak \#

Start Tube

End Tube

\begin{tabular}{c|c} 
A:3 & A:3 \\
A:4 & A:4 \\
A:5 & A:5 \\
A:6 & A:6 \\
A:7 & A:8 \\
A:11 & A:11 \\
A:12 & A:12 \\
A:13 & A:13 \\
A:15 & A:15 \\
$\cdots$ & $\cdots$
\end{tabular}

\begin{tabular}{|c|c|c|c|}
\hline Duration & \multicolumn{1}{c}{ \% B } & \multicolumn{1}{c|}{ Solvent A } & Solvent B \\
\hline $\mathbf{0 . 0}$ & $\mathbf{0 . 0}$ & A2 dichlorometha & B2 methanol \\
$\mathbf{1 2 . 5}$ & $\mathbf{8 . 4}$ & A2 dichlorometha & B2 methanol \\
& & & \\
& & & \\
& & & \\
& & & \\
& & & \\
& & & \\
\hline
\end{tabular}


Figure S8. 5,6-Diphenyl-3-[6-(5-p-tolyl-2H-pyrazol-3-yl)-pyridin-2-yl]-[1,2,4]triazine (2)

Sample: VGB-A-283

Rf 200

Friday 02 November 2018 11:41AM

4x Column: 3

RediSep Column: Al2O3 pH=7 8g

Flow Rate: $18 \mathrm{ml} / \mathrm{min}$

Equilibration Volume: $33.6 \mathrm{ml}$

Initial Waste: $0.0 \mathrm{ml}$

Air Purge: 0.5 min

Solvent: A2 dichloromethane

Solvent: B2 2-propanol
Peak Tube Volume: Max.

Non-Peak Tube Volume: Max.

Loading Type: Solid

Wavelength 1 (red): 254nm

Peak Width: 30 sec

Threshold: 0.20 AU

Wavelength 2 (purple): 280nm

Run Notes:

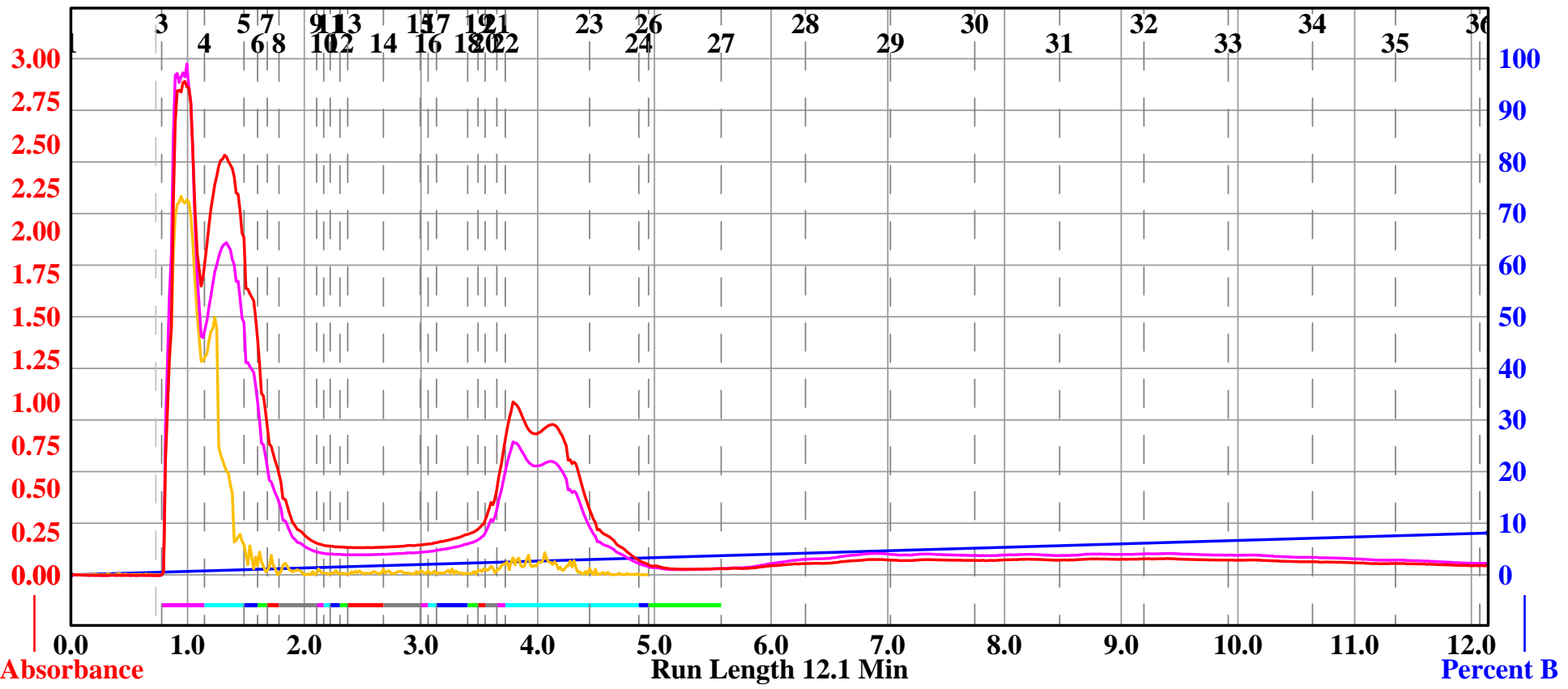

Rack A

\begin{tabular}{|c|c|c|c|}
\hline (71) & (72) & (13) & (14) \\
\hline (70) & (69) & (68) & (67) \\
\hline (61) & (62) & (63) & (64) \\
\hline (60) & (59) & (58) & (57) \\
\hline (51) & (52) & (53) & (54) \\
\hline (50) & (49) & (48) & (47) \\
\hline (41) & (42) & (43) & (44) \\
\hline (40) & (39) & (38) & (37) \\
\hline (31) & (32) & (33) & (34) \\
\hline (30) & (29) & (28) & (27) \\
\hline (21) & (22) & (23) & \\
\hline (20) & (19) & (18) & \\
\hline (1) & (12) & (13) & (14) \\
\hline (10) & (9) & (8) & (7) \\
\hline (1) & (2) & (3) & (4) \\
\hline
\end{tabular}

$16 \mathrm{~mm} \times 100 \mathrm{~mm}$ Tubes

\begin{tabular}{|c|c|c|c|}
\hline \multicolumn{2}{|c|}{ Peak \# } & Start Tube & End Tube \\
\hline \multicolumn{2}{|l|}{1} & A:3 & $A: 3$ \\
\hline \multicolumn{2}{|l|}{2} & A:4 & A:4 \\
\hline \multicolumn{2}{|l|}{3} & A:5 & A:5 \\
\hline \multicolumn{2}{|l|}{4} & A:6 & A:6 \\
\hline \multicolumn{2}{|l|}{5} & A:7 & A:7 \\
\hline \multicolumn{2}{|l|}{6} & A:8 & A:8 \\
\hline \multicolumn{2}{|l|}{7} & A:9 & A:9 \\
\hline \multicolumn{2}{|l|}{8} & A:10 & A:10 \\
\hline \multicolumn{2}{|l|}{9} & A:11 & A:11 \\
\hline \multicolumn{2}{|l|}{$\cdots$} & $\cdots$ & $\cdots$ \\
\hline Duration & $\% \mathrm{~B}$ & Solvent A & Solvent B \\
\hline 0.0 & 0.0 & A2 dichlorometha & B2 methanol \\
\hline 12.1 & 8.1 & A2 dichlorometha & B2 methanol \\
\hline & & & \\
\hline & & & \\
\hline & & & \\
\hline & & & \\
\hline
\end{tabular}


Figure S9. 3-\{6-[5-(4-Fluoro-phenyl)-2H-pyrazol-3-yl]-pyridin-2-yl\}-5,6-diphenyl-[1,2,4]triazine (10) Sample: VGB-B-225

4x Column: 2

RediSep Column: Silica 4g

Flow Rate: $18 \mathrm{ml} / \mathrm{min}$

Equilibration Volume: $33.6 \mathrm{ml}$

Initial Waste: $0.0 \mathrm{ml}$

Air Purge: 1.0 min

Solvent: $\mathrm{Al}_{\mathrm{CH}_{3} \mathrm{CN}}$
Peak Tube Volume: Max.

Non-Peak Tube Volume: Max.

Loading Type: Solid

Wavelength 1 (red): 254nm

Peak Width: $30 \mathrm{sec}$

Threshold: 0.20 AU

Wavelength 2 (purple): 280nm
All Wavelength (orange): 200-300nm

Peak Width: 30 sec

Threshold: 0.20 AU

Run Notes:

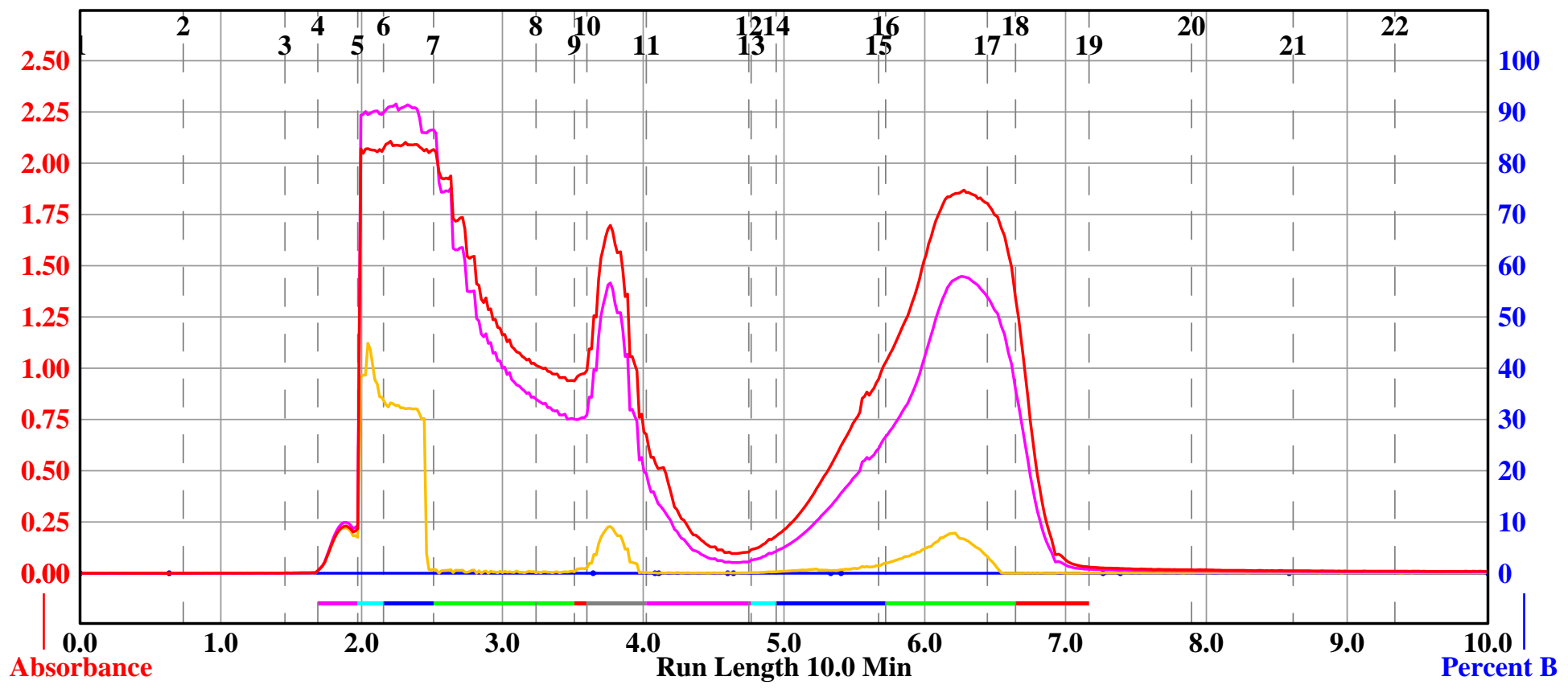

Rack A

\begin{tabular}{|c|c|c|c|}
\hline (1) & (72) & (13) & (74) \\
\hline (70) & (6) & (68) & (67) \\
\hline 61 & (62) & (63) & (64) \\
\hline (60) & (59) & (58) & (57) \\
\hline 51 & (52) & (33) & (54) \\
\hline (30) & (49) & (48) & (47) \\
\hline & (42) & (43) & (44) \\
\hline & (39) & (38) & (37) \\
\hline (31) & (32) & (33) & (34) \\
\hline & (29) & (28) & (27) \\
\hline & (22) & (23) & (24) \\
\hline & (19) & (18) & (1) \\
\hline & (12) & (13) & \\
\hline & (9) & (8) & 7 \\
\hline & (2) & (3) & (4) \\
\hline
\end{tabular}

$16 \mathrm{~mm} \times 100 \mathrm{~mm}$ Tubes

Peak \#

Start Tube

End Tube

\begin{tabular}{|c|c|c|c|}
\hline 1 & & A:4 & A:4 \\
\hline 2 & & A:5 & A:5 \\
\hline 3 & & A:6 & A:6 \\
\hline 4 & & A:7 & A:8 \\
\hline 5 & & A:9 & A:9 \\
\hline 6 & & A:10 & A:10 \\
\hline 7 & & A:11 & A:12 \\
\hline 8 & & A:13 & A:13 \\
\hline 9 & & A:14 & A:15 \\
\hline ... & & $\cdots$ & $\cdots$ \\
\hline Duration & $\% \mathbf{B}$ & Solvent A & Solvent B \\
\hline 0.0 & 0.0 & A1 hexane & B1 ethyl acetate \\
\hline 0.6 & 0.0 & A1 hexane & B1 ethyl acetate \\
\hline 3.0 & 0.0 & A1 hexane & B1 ethyl acetate \\
\hline 0.4 & 0.0 & A1 hexane & B1 ethyl acetate \\
\hline 0.0 & 0.0 & A1 hexane & B1 ethyl acetate \\
\hline 0.5 & 0.0 & A1 hexane & B1 ethyl acetate \\
\hline 0.0 & 0.0 & A1 hexane & B1 ethyl acetate \\
\hline 0.7 & 0.0 & A1 hexane & B1 ethyl acetate \\
\hline 0.1 & 0.0 & A1 hexane & B1 ethyl acetate \\
\hline 1.9 & 0.0 & A1 hexane & B1 ethyl acetate \\
\hline 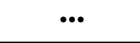 & $\ldots$ & ... & \\
\hline
\end{tabular}


Figure S10. 5,6-Diphenyl-3-\{6-[5-(4-trifluoromethyl-phenyl)-2H-pyrazol-3-yl]-pyridin-2-yl\}-[1,2,4]triazine (11) Sample: VGB-B-251

Rf 200

Peak Tube Volume: Max.

4x Column: 2

RediSep Column: Silica $4 \mathrm{~g}$

Flow Rate: $18 \mathrm{ml} / \mathrm{min}$

Equilibration Volume: $33.6 \mathrm{ml}$

Initial Waste: $0.0 \mathrm{ml}$

Air Purge: 0.5 min

Solvent: $\mathrm{Al}_{\mathrm{CH}_{3} \mathrm{CN}}$
Non-Peak Tube Volume: Max.

Loading Type: Solid

Wavelength 1 (red): 254nm

Peak Width: 30 sec

Threshold: 0.20 AU

Wavelength 2 (purple): 280nm
Wednesday 23 January 2019 06:00PM

All Wavelength (orange): 200-300nm

Peak Width: 30 sec

Threshold: 0.20 AU

Run Notes:

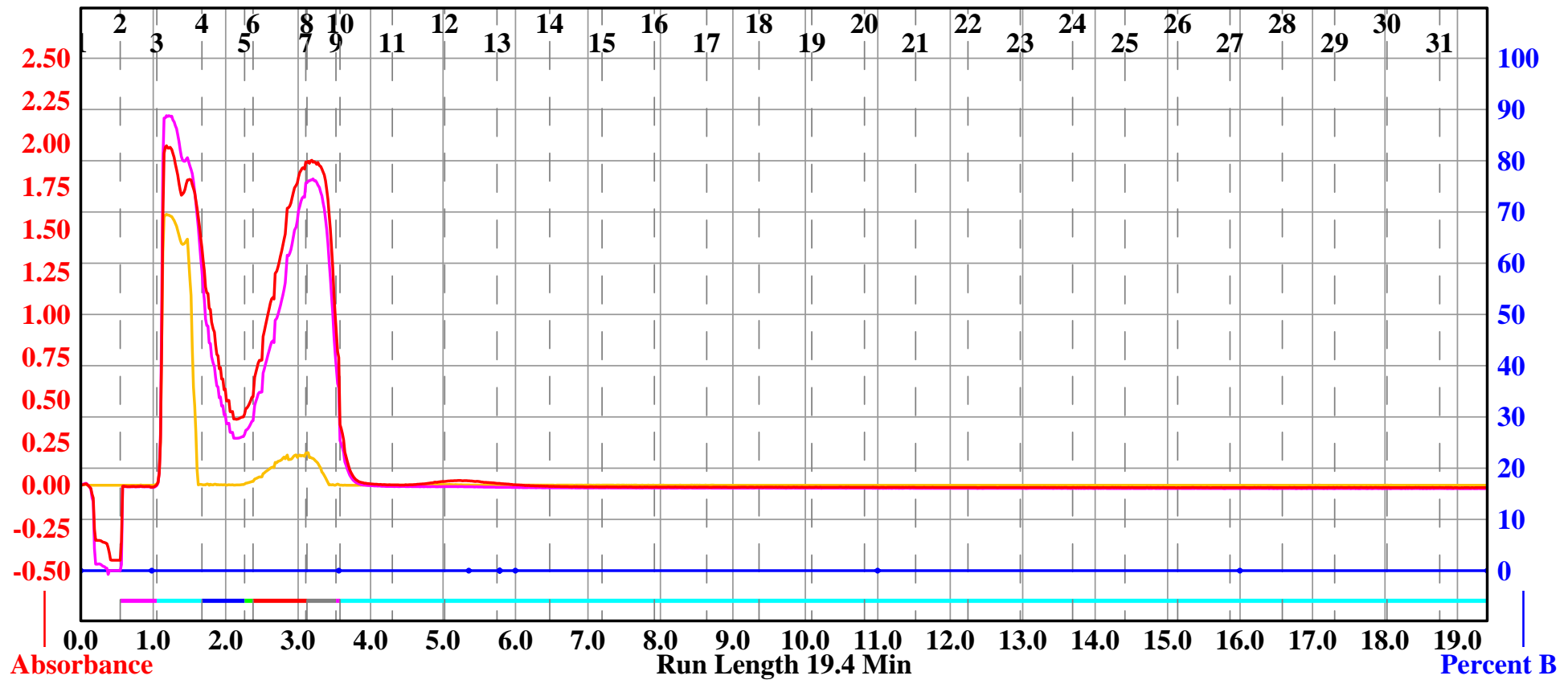

Rack A

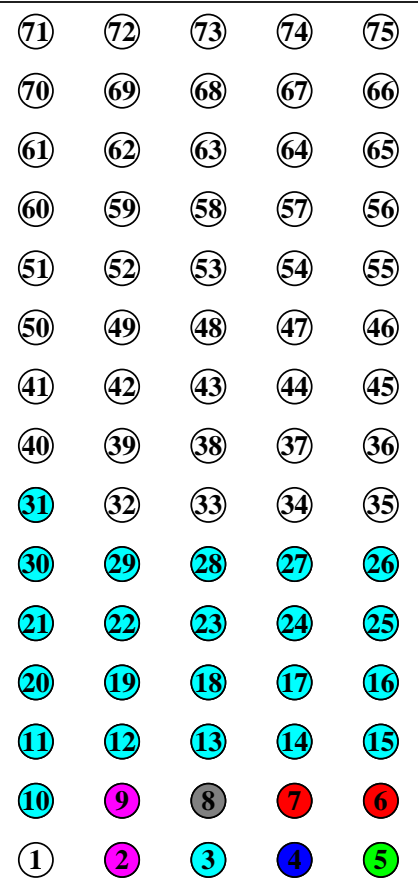

$16 \mathrm{~mm} \times 100 \mathrm{~mm}$ Tubes

Start Tube $\quad$ End Tube

\begin{tabular}{|c|c|c|c|}
\hline $\begin{array}{l}1 \\
2 \\
3 \\
4 \\
5 \\
6 \\
7 \\
8\end{array}$ & & $\begin{array}{c}\text { A:2 } \\
\text { A:3 } \\
\text { A:4 } \\
\text { A:5 } \\
\text { A:6 } \\
\text { A:8 } \\
\text { A:9 } \\
\text { A:10 }\end{array}$ & $\begin{array}{r}\text { A:2 } \\
\text { A:3 } \\
\text { A:4 } \\
\text { A:5 } \\
\text { A:7 } \\
\text { A:8 } \\
\text { A:9 } \\
\text { A:31 }\end{array}$ \\
\hline Duration & $\%$ B & Solvent A & Solvent B \\
\hline $\begin{array}{l}.0 \\
1.0 \\
2.6 \\
1.8 \\
0.4 \\
0.0 \\
0.2 \\
5.0 \\
5.0 \\
3.4\end{array}$ & $\begin{array}{l}\text { 0.0 } \\
\text { 0.0 } \\
\text { 0.0 } \\
\text { 0.0 } \\
\text { 0.0 } \\
\text { 0.0 } \\
\text { 0.0 } \\
\text { 0.0 } \\
\text { 0.0 }\end{array}$ & $\begin{array}{l}\text { A1 hexane } \\
\text { A1 hexane } \\
\text { A1 hexane } \\
\text { A1 hexane } \\
\text { A1 hexane } \\
\text { A1 hexane } \\
\text { A1 hexane } \\
\text { A1 hexane } \\
\text { A1 hexane } \\
\text { A1 hexane }\end{array}$ & $\begin{array}{l}\text { B1 ethyl acetate } \\
\text { B1 ethyl acetate } \\
\text { B1 ethyl acetate } \\
\text { B1 ethyl acetate } \\
\text { B1 ethyl acetate } \\
\text { B1 ethyl acetate } \\
\text { B1 ethyl acetate } \\
\text { B1 ethyl acetate } \\
\text { B1 ethyl acetate } \\
\text { B1 ethyl acetate }\end{array}$ \\
\hline
\end{tabular}


Figure S11. 3-\{6-[5-(4-Bromo-phenyl)-2H-pyrazol-3-yl]-pyridin-2-yl\}-5,6-diphenyl-[1,2,4]triazine (12)
Sf 200

4x Column: 2

RediSep Column: Silica 4g

Flow Rate: $18 \mathrm{ml} / \mathrm{min}$

Equilibration Volume: $33.6 \mathrm{ml}$

Initial Waste: $0.0 \mathrm{ml}$

Air Purge: $0.5 \mathrm{~min}$

Solvent: $\mathrm{A} 1 \mathrm{CH}_{3} \mathrm{CN}$
Peak Tube Volume: Max.

Non-Peak Tube Volume: Max.

Loading Type: Solid

Wavelength 1 (red): 254nm

Peak Width: $30 \mathrm{sec}$

Threshold: 0.20 AU

Wavelength 2 (purple): 280nm
All Wavelength (orange): $200-300 \mathrm{~nm}$

Peak Width: $30 \mathrm{sec}$

Threshold: 0.20 AU

Run Notes:

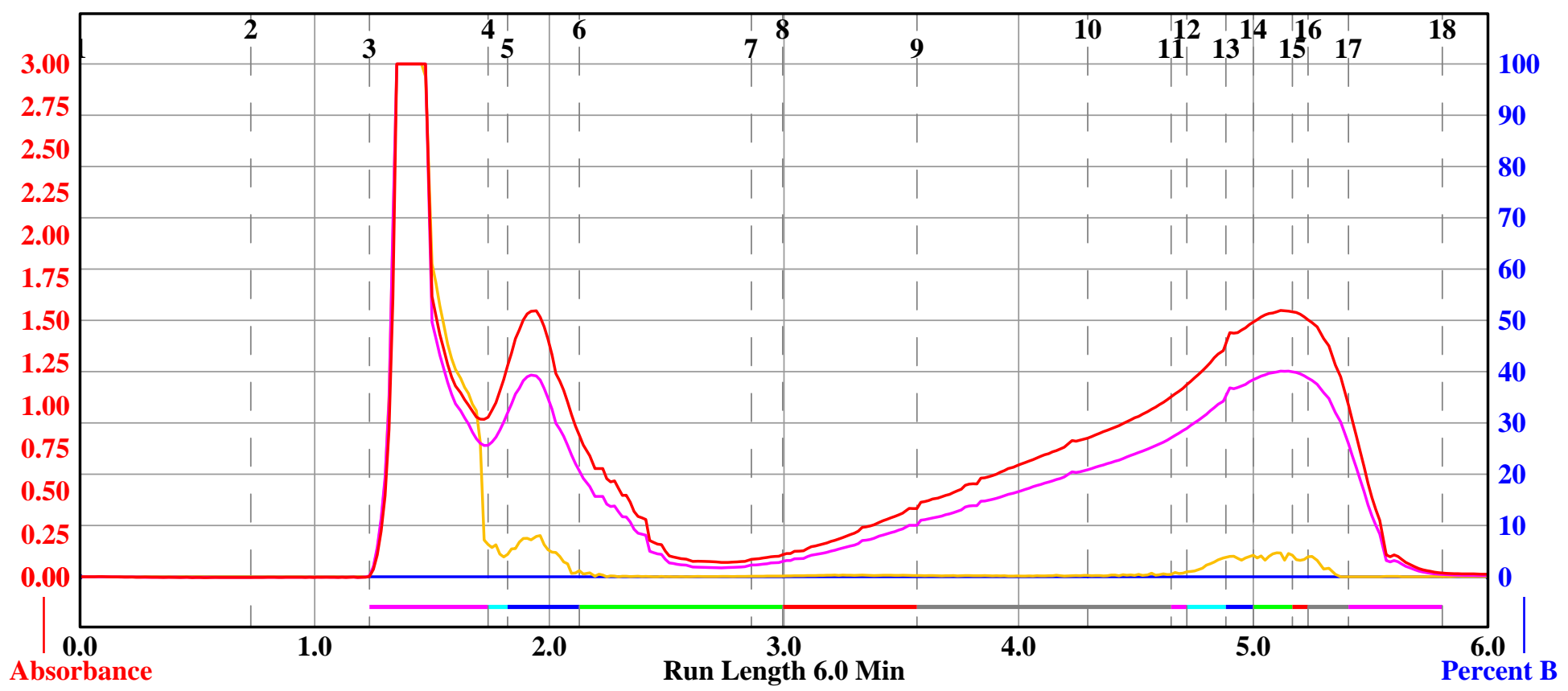

Rack A

\begin{tabular}{|c|c|c|c|}
\hline (71) & (72) & (13) & (74) \\
\hline (70) & (69) & (68) & (67) \\
\hline (61) & (62) & (63) & (64) \\
\hline (60) & (59) & (58) & (57) \\
\hline (51) & (52) & (53) & (54) \\
\hline (50) & (49) & (48) & (47) \\
\hline (41) & (42) & (43) & (44) \\
\hline (40) & (39) & (38) & (37) \\
\hline (31) & (32) & (33) & (34) \\
\hline (30) & (29) & (28) & (27) \\
\hline (21) & (22) & (23) & (24) \\
\hline (20) & (19) & (18) & (17) \\
\hline (11) & (12) & & (14) \\
\hline (10) & (9) & 8 & (7) \\
\hline (1) & (2) & (3) & (4) \\
\hline
\end{tabular}

$16 \mathrm{~mm} \times 100 \mathrm{~mm}$ Tubes

Peak \#

Start Tube

End Tube

\begin{tabular}{|c|c|c|c|}
\hline $\begin{array}{l}1 \\
2 \\
3 \\
3 \\
4 \\
5 \\
6 \\
7 \\
8 \\
8 \\
9 \\
\cdots\end{array}$ & & $\begin{array}{c}\text { A:3 } \\
\text { A:4 } \\
\text { A:5 } \\
\text { A:6 } \\
\text { A:8 } \\
\text { A:9 } \\
\text { A:11 } \\
\text { A:12 } \\
\text { A:13 } \\
\text {... }\end{array}$ & $\begin{array}{c}\text { A:3 } \\
\text { A:4 } \\
\text { A:5 } \\
\text { A:7 } \\
\text { A:8 } \\
\text { A:10 } \\
\text { A:11 } \\
\text { A:12 } \\
\text { A:13 } \\
\text {... }\end{array}$ \\
\hline Duration & $\%$ В & Solvent A & Solvent B \\
\hline $\begin{array}{l}0.0 \\
6.0\end{array}$ & $\begin{array}{l}0.0 \\
0.0\end{array}$ & $\begin{array}{l}\text { A1 hexane } \\
\text { A1 hexane }\end{array}$ & $\begin{array}{l}\text { B1 ethyl acetate } \\
\text { B1 ethyl acetate }\end{array}$ \\
\hline
\end{tabular}


Figure S12. (4-\{5-[6-(5,6-Diphenyl-[1,2,4]triazin-3-yl)-pyridin-2-yl]-1H-pyrazol-3-yl\}-phenyl)-dimethyl (13) Sample: VGB-C-17

Rf 200

4x Column: 1

RediSep Column: Silica 4g

Flow Rate: $18 \mathrm{ml} / \mathrm{min}$

Equilibration Volume: $33.6 \mathrm{ml}$

Initial Waste: $0.0 \mathrm{ml}$

Air Purge: 0.5 min

Solvent: $\mathrm{A} 1 \mathrm{CH}_{3} \mathrm{CN}$
Peak Tube Volume: Max.

Non-Peak Tube Volume: Max.

Loading Type: Solid

Wavelength 1 (red): 254nm

Peak Width: 30 sec

Threshold: $0.20 \mathrm{AU}$

Wavelength 2 (purple): 280nm
Tuesday 05 March 2019 10:24AM

All Wavelength (orange): 200-300nm

Peak Width: 30 sec

Threshold: 0.20 AU

Run Notes:

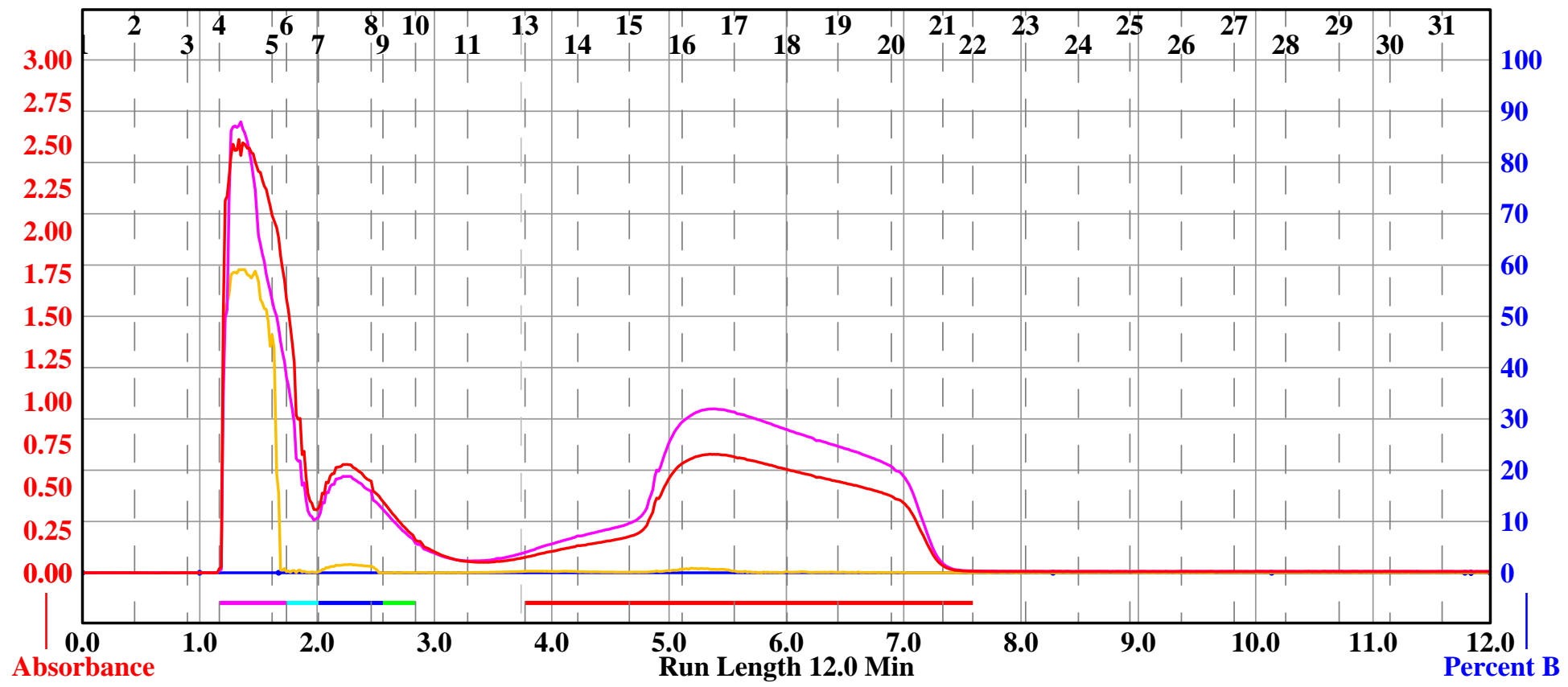

Rack A

Start Tube

End Tube

\begin{tabular}{|c|c|c|c|c|c|}
\hline & (107 & 106 & 105 & (104 & 10 \\
\hline & $\mathscr{B}$ & (9) & (10) & (1) & \\
\hline & (פ) & (4) & (2) & 22 & \\
\hline 35 & 8 & 87 & 88 & 89 & \\
\hline & $\$ 3$ & 82 & 81 & B0 & \\
\hline & 87 & 18 & (ש) & (17) & \\
\hline 12 & (1) & (10) & (6) & 68 & \\
\hline & (2) & (3) & (3) & 6 & \\
\hline (1) & 59 & 58 & 57 & b & \\
\hline 9 & (1) & (1) & 52 & 53 & \\
\hline 18 & (47) & (46) & 48 & (4) & \\
\hline 37 & B8 & (39) & (41) & (41) & \\
\hline ほf & BS & B & $\mathfrak{B}$ & (12) & \\
\hline & dd & 28 & $\mathfrak{B}$ & 29 & \\
\hline (24) & 23 & 22 & e & 20 & \\
\hline & $\mathbf{v}$ & (5) & 0 & 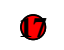 & \\
\hline C & (1) & (10) & (D) & & \\
\hline & (2) & (3) & (4) & (5) & \\
\hline
\end{tabular}

$13 \mathrm{~mm}$ x $100 \mathrm{~mm}$ Tubes

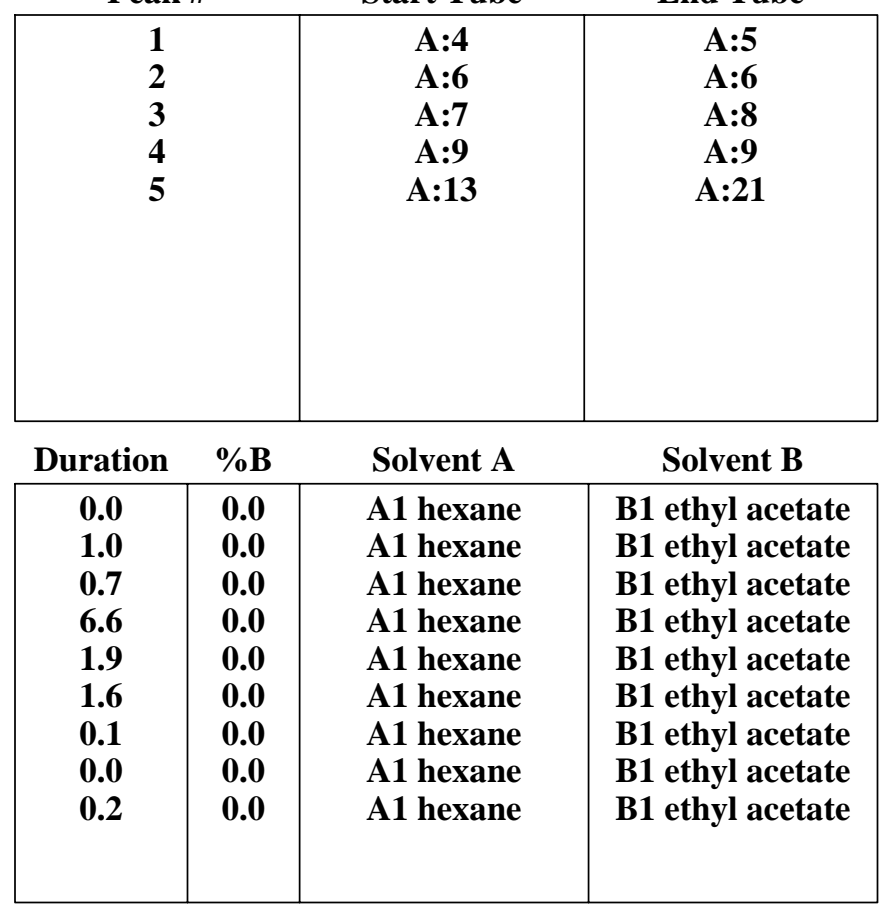


Figure S13. 3-\{6-[5-(4-Methoxy-phenyl)-2H-pyrazol-3-yl]-pyridin-2-yl\}-5,6-diphenyl-[1,2,4]triazine (14) Sample: VGB-B-203

4x Column: 2

RediSep Column: Silica $4 \mathrm{~g}$

Flow Rate: $18 \mathrm{ml} / \mathrm{min}$

Equilibration Volume: $33.6 \mathrm{ml}$

Initial Waste: $0.0 \mathrm{ml}$

Air Purge: 1.0 min

Solvent: $\mathrm{A} 1 \mathrm{CH}_{3} \mathrm{CN}$
Peak Tube Volume: Max.

Non-Peak Tube Volume: Max.

Loading Type: Solid

Wavelength 1 (red): 254nm

Peak Width: 30 sec

Threshold: $0.20 \mathrm{AU}$

Wavelength 2 (purple): 280nm
All Wavelength (orange): $200-300 \mathrm{~nm}$

Peak Width: 30 sec

Threshold: 0.20 AU

Run Notes:

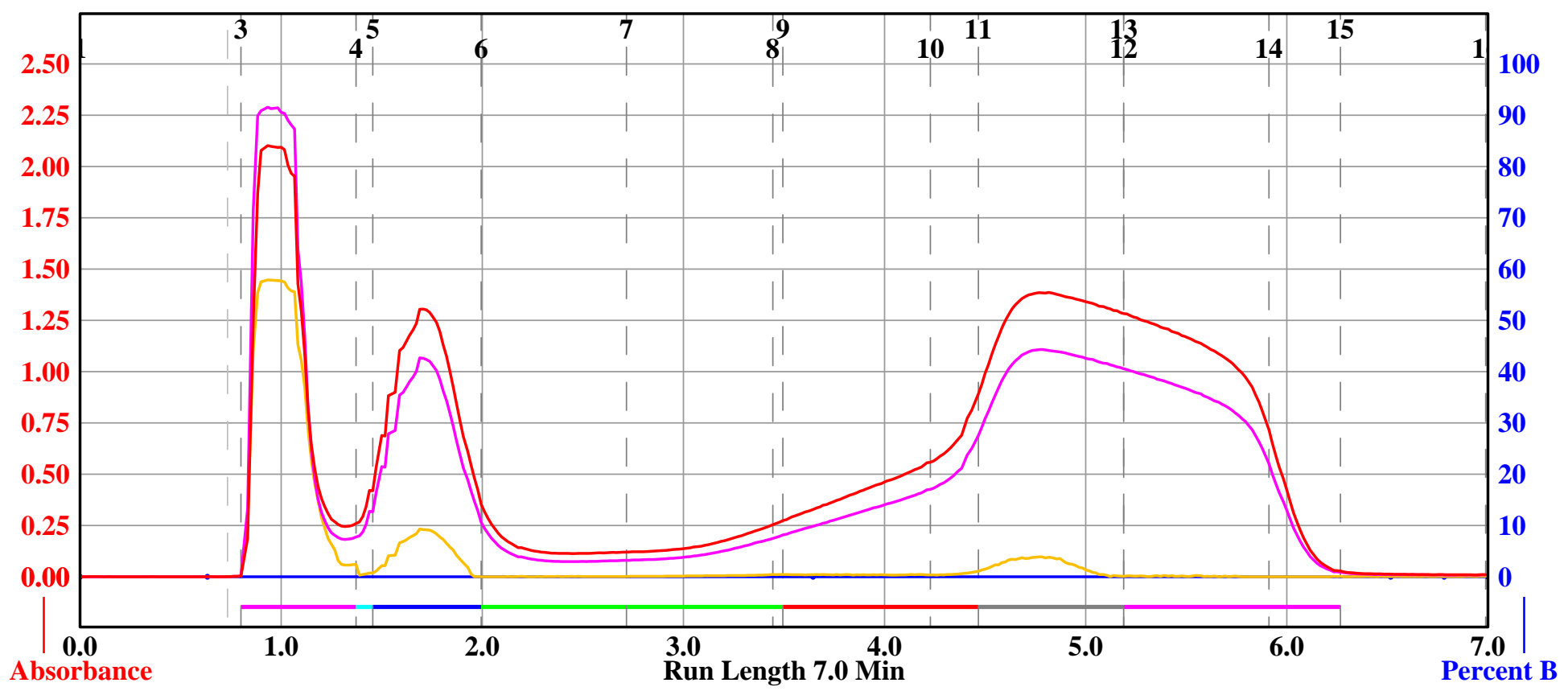

Rack A

\begin{tabular}{|c|c|c|c|c|}
\hline (71) & (72) & (73) & (74) & (15) \\
\hline (70) & (69) & (68) & (67) & (66) \\
\hline (61) & (62) & (6) & (64) & (65) \\
\hline (6) & (59) & (58) & (57) & (56) \\
\hline (51) & (52) & (53) & (54) & (55) \\
\hline (50) & (49) & (48) & (47) & (46) \\
\hline (41) & (42) & (43) & (44) & (45) \\
\hline (40) & (39) & (38) & (37) & (36) \\
\hline (31) & (32) & (33) & (34) & (35) \\
\hline (30) & (29) & (28) & (27) & (26) \\
\hline (21) & (22) & (23) & (24) & (25) \\
\hline 20 & (19) & (18) & (17) & (16) \\
\hline (11) & (12) & (13) & (14) & (15) \\
\hline & (9) & (8) & (7) & (6) \\
\hline (1) & (2) & (3) & (4) & \\
\hline
\end{tabular}

$16 \mathrm{~mm} \times 100 \mathrm{~mm}$ Tubes

Peak \#

Start Tube

End Tube

\begin{tabular}{|c|c|c|c|}
\hline $\begin{array}{l}1 \\
2 \\
3 \\
4 \\
5 \\
5 \\
6 \\
7\end{array}$ & & $\begin{array}{l}\text { A:3 } \\
\text { A:4 } \\
\text { A:5 } \\
\text { A:6 } \\
\text { A:9 } \\
\text { A:11 } \\
\text { A:13 }\end{array}$ & $\begin{array}{l}\text { A:3 } \\
\text { A:4 } \\
\text { A:5 } \\
\text { A:8 } \\
\text { A:10 } \\
\text { A:12 } \\
\text { A:14 }\end{array}$ \\
\hline Duration & $\%$ В & Solvent A & Solvent B \\
\hline $\begin{array}{l}0.0 \\
0.6 \\
3.0 \\
2.9 \\
0.3 \\
0.2\end{array}$ & $\begin{array}{l}0.0 \\
0.0 \\
0.0 \\
0.0 \\
\text { 0.0 } \\
\mathbf{0 . 0}\end{array}$ & $\begin{array}{l}\text { A1 hexane } \\
\text { A1 hexane } \\
\text { A1 hexane } \\
\text { A1 hexane } \\
\text { A1 hexane } \\
\text { A1 hexane }\end{array}$ & $\begin{array}{l}\text { B1 ethyl acetate } \\
\text { B1 ethyl acetate } \\
\text { B1 ethyl acetate } \\
\text { B1 ethyl acetate } \\
\text { B1 ethyl acetate } \\
\text { B1 ethyl acetate }\end{array}$ \\
\hline
\end{tabular}


Figure S14. 3-\{5-[6-(5,6-Diphenyl-[1,2,4]triazin-3-yl)-pyridin-2-yl]-1H-pyrazol-3-yl\}-benzoic methyl ester (15) Sample: VGB-B-215

4x Column: 2

RediSep Column: Silica 4g

Flow Rate: $18 \mathrm{ml} / \mathrm{min}$

Equilibration Volume: $33.6 \mathrm{ml}$

Initial Waste: $0.0 \mathrm{ml}$

Air Purge: $1.0 \mathrm{~min}$

Solvent: $\mathrm{A} 1 \mathrm{CH}_{3} \mathrm{CN}$
Peak Tube Volume: Max.

Non-Peak Tube Volume: Max.

Loading Type: Solid

Wavelength 1 (red): $254 \mathrm{~nm}$

Peak Width: 30 sec

Threshold: $0.20 \mathrm{AU}$

Wavelength 2 (purple): $280 \mathrm{~nm}$
Monday 14 January 2019 11:17AM

All Wavelength (orange): 200-300nm

Peak Width: 30 sec

Threshold: 0.20 AU

Run Notes:

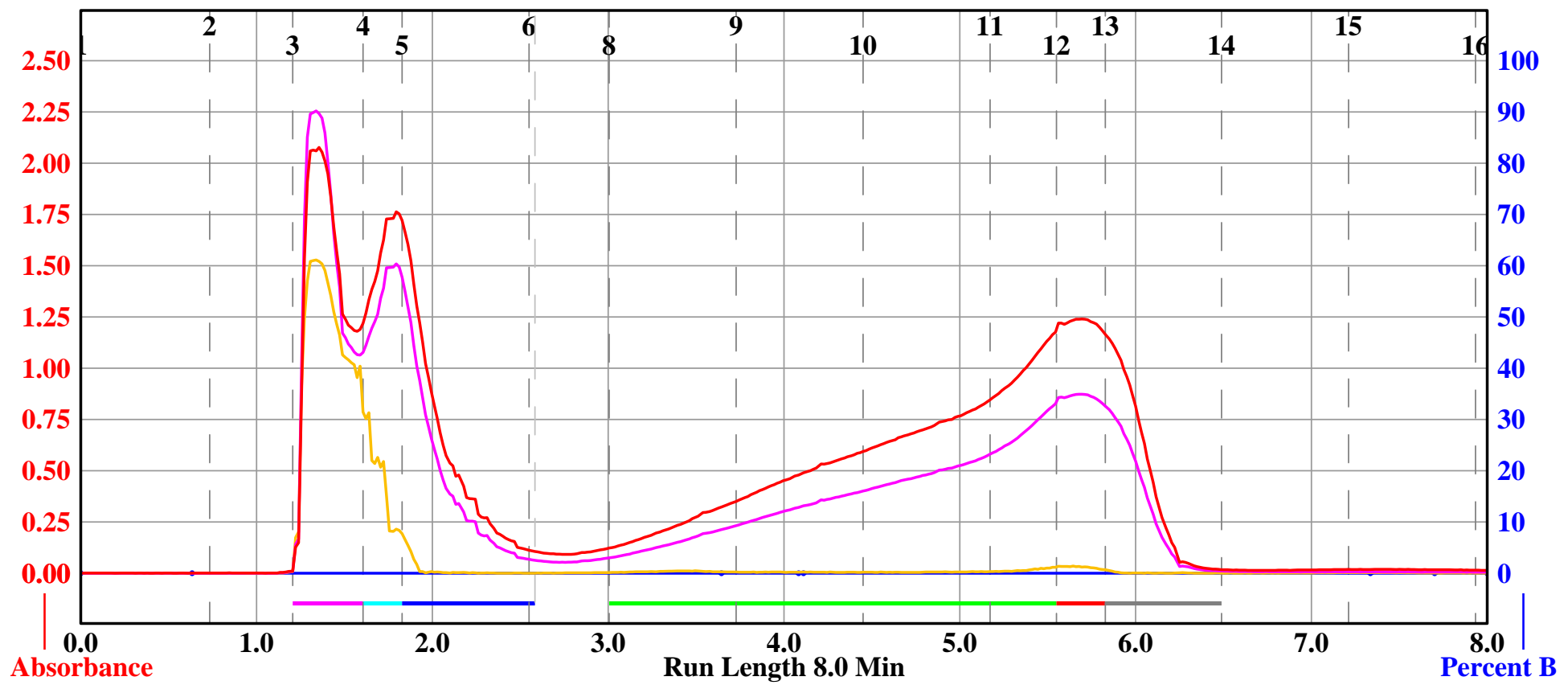

Rack A

Peak

Start Tube

End Tube

\begin{tabular}{|c|c|c|c|}
\hline (71) & (12) & (73) & (74) \\
\hline (71) & (9) & (88) & (67) \\
\hline (61) & (62) & (63) & (44) \\
\hline (60) & (59) & (88) & (57) \\
\hline (11) & (22) & (33) & (44) \\
\hline (50) & (49) & (48) & (47) \\
\hline (41) & (42) & (43) & (44) \\
\hline (40) & (39) & (38) & (37) \\
\hline (31) & (22) & (33) & (44) \\
\hline (30) & (29) & (28) & (27) \\
\hline (21) & (22) & (23) & (24) \\
\hline (20) & (19) & (18) & (17) \\
\hline (11) & (12) & (13) & (14) \\
\hline (10) & (9) & (8) & (7) \\
\hline (1) & (2) & (3) & (4) \\
\hline
\end{tabular}

$16 \mathrm{~mm} \times 100 \mathrm{~mm}$ Tubes

\begin{tabular}{|c|c|c|c|}
\hline $\begin{array}{l}1 \\
2 \\
3 \\
4 \\
5 \\
6\end{array}$ & & $\begin{array}{c}A: 3 \\
A: 4 \\
A: 5 \\
A: 8 \\
A: 12 \\
A: 13\end{array}$ & $\begin{array}{c}A: 3 \\
A: 4 \\
A: 6 \\
A: 11 \\
A: 12 \\
A: 13\end{array}$ \\
\hline Duration & $\%$ B & Solvent A & Solvent B \\
\hline $\begin{array}{l}0.0 \\
0.6 \\
3.0 \\
0.4 \\
0.0 \\
3.2 \\
0.4 \\
0.3\end{array}$ & $\begin{array}{l}\mathbf{0 . 0} \\
\mathbf{0 . 0} \\
\mathbf{0 . 0} \\
\mathbf{0 . 0} \\
\mathbf{0 . 0} \\
\mathbf{0 . 0} \\
\mathbf{0 . 0} \\
\mathbf{0 . 0}\end{array}$ & $\begin{array}{l}\text { A1 hexane } \\
\text { A1 hexane } \\
\text { A1 hexane } \\
\text { A1 hexane } \\
\text { A1 hexane } \\
\text { A1 hexane } \\
\text { A1 hexane } \\
\text { A1 hexane }\end{array}$ & $\begin{array}{l}\text { B1 ethyl acetate } \\
\text { B1 ethyl acetate } \\
\text { B1 ethyl acetate } \\
\text { B1 ethyl acetate } \\
\text { B1 ethyl acetate } \\
\text { B1 ethyl acetate } \\
\text { B1 ethyl acetate } \\
\text { B1 ethyl acetate }\end{array}$ \\
\hline
\end{tabular}


Figure S15. 3-\{6-[5-(2-lodo-phenyl)-2H-pyrazol-3-yl]-pyridin-2-yl\}-5,6-diphenyl-[1,2,4]triazine (16) Sample: VGB-B-249

4x Column: 2

RediSep Column: Silica 4g

Flow Rate: $18 \mathrm{ml} / \mathrm{min}$

Equilibration Volume: $33.6 \mathrm{ml}$

Initial Waste: $0.0 \mathrm{ml}$

Air Purge: 0.5 min

Solvent: $\mathrm{A} 1 \mathrm{CH}_{3} \mathrm{CN}$
Peak Tube Volume: Max.

Non-Peak Tube Volume: Max.

Loading Type: Solid

Wavelength 1 (red): 254nm

Peak Width: $30 \mathrm{sec}$

Threshold: 0.20 AU

Wavelength 2 (purple): 280nm
All Wavelength (orange): $200-300 \mathrm{~nm}$

Peak Width: 30 sec

Threshold: 0.20 AU

Run Notes:

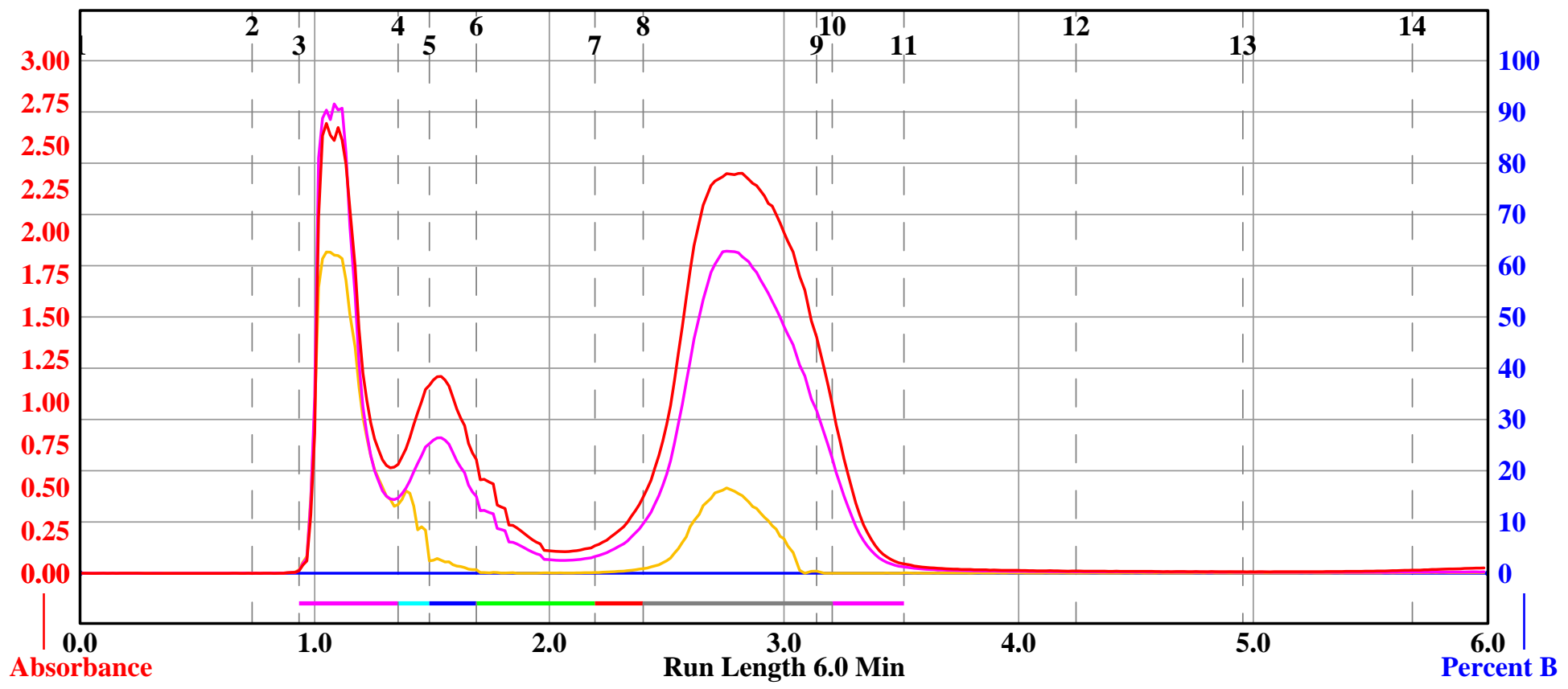

Rack A

\begin{tabular}{|c|c|c|c|}
\hline (11) & (72) & (73) & (74) \\
\hline (70) & (69) & (68) & (67) \\
\hline 61) & (62) & (63) & (64) \\
\hline (0) & (59) & (58) & (57) \\
\hline 51 & (52) & (53) & (54) \\
\hline 3 & (49) & (48) & (47) \\
\hline & (42) & (43) & (44) \\
\hline & (39) & (38) & (37) \\
\hline & (32) & (33) & (34) \\
\hline & (29) & (28) & (27) \\
\hline & (22) & (23) & (24) \\
\hline & (19) & (18) & (17) \\
\hline & (12) & (13) & (14) \\
\hline & (9) & (8) & (7) \\
\hline & (2) & (3) & (4) \\
\hline
\end{tabular}

$16 \mathrm{~mm}$ x $100 \mathrm{~mm}$ Tubes

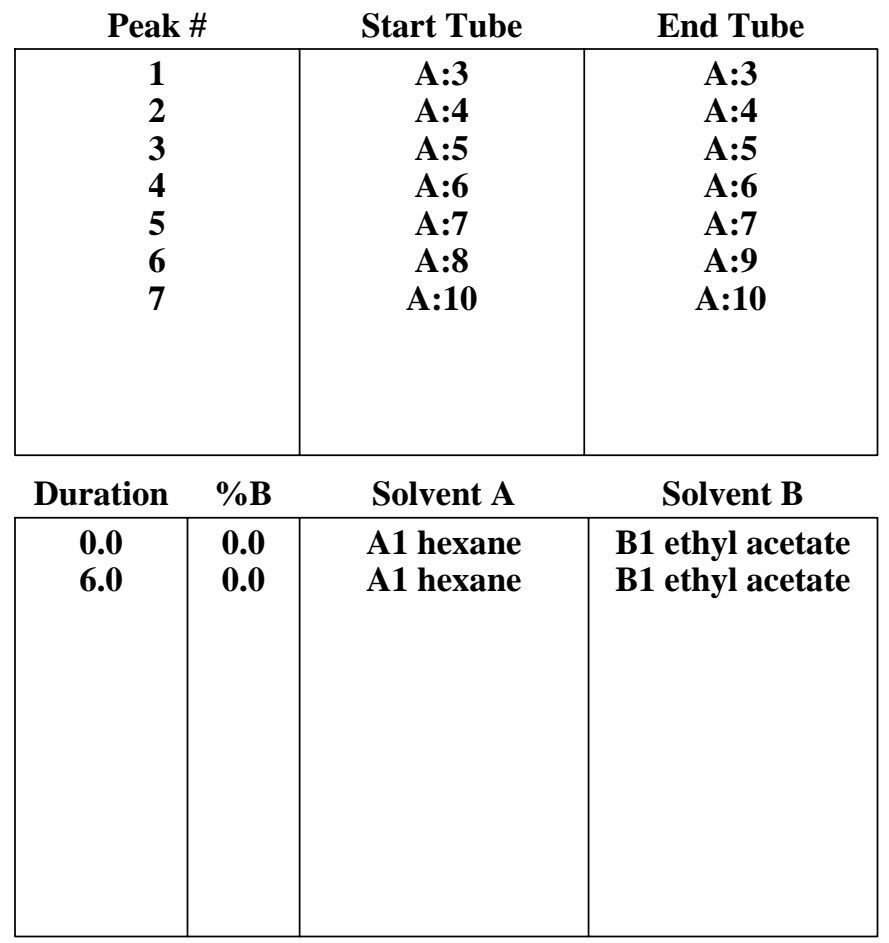


Figure S16. 5,6-Diphenyl-3-\{6-[5-(2,4,6-trifluoro-phenyl)-2H-pyrazol-3-yl]-pyridin-2-yl\}-[1,2,4]triazine (17) Sample: VGB-B-61

Wednesday 14 November 2018 08:24PM

4x Column: 3

RediSep Column: Silica 4g

Flow Rate: $18 \mathrm{ml} / \mathrm{min}$

Equilibration Volume: $33.6 \mathrm{ml}$

Initial Waste: $0.0 \mathrm{ml}$

Air Purge: $0.5 \mathrm{~min}$

Solvent: A2 dichloromethane

Solvent: B2 2-propanol
Peak Tube Volume: Max.

Non-Peak Tube Volume: Max.

Loading Type: Solid

Wavelength 1 (red): 254nm

Peak Width: $30 \mathrm{sec}$

Threshold: 0.20 AU

Wavelength 2 (purple): 280nm
All Wavelength (orange): 200-300nm

Peak Width: 30 sec

Threshold: 0.20 AU

Run Notes:

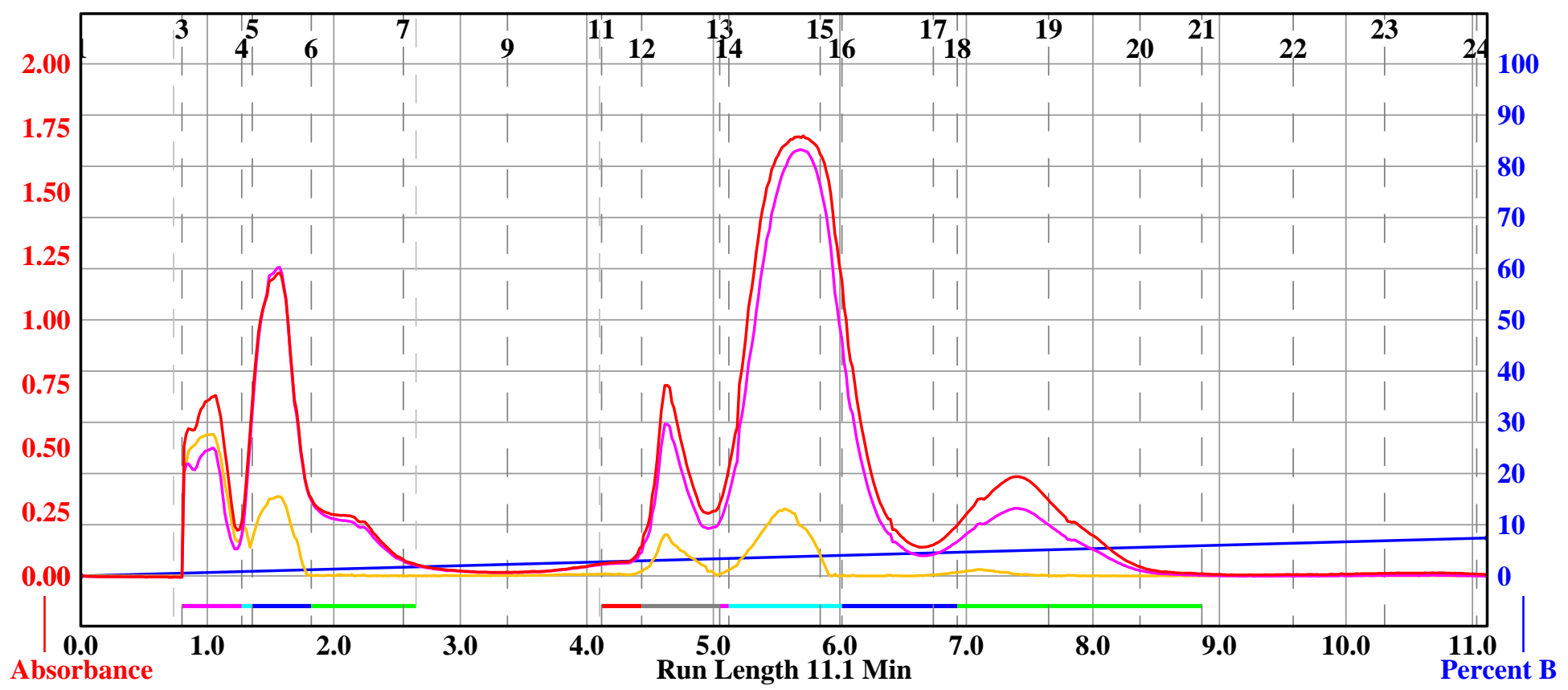

Rack A

\begin{tabular}{|c|c|c|c|}
\hline (71) & (72) & (13) & (74) \\
\hline (70) & (69) & (68) & (67) \\
\hline (61) & (62) & (63) & (64) \\
\hline (60) & (59) & (58) & (57) \\
\hline (51) & (52) & (53) & (54) \\
\hline (50) & (49) & (48) & (47) \\
\hline (41) & (42) & (43) & (44) \\
\hline (40) & (39) & (38) & (37) \\
\hline (31) & (32) & (33) & (34) \\
\hline (30) & (29) & (28) & (27) \\
\hline (21) & (22) & (23) & (24) \\
\hline (20) & (19) & (18) & (1) \\
\hline (1) & (12) & (13) & (14) \\
\hline (10) & (9) & (8) & (7) \\
\hline (1) & (2) & (3) & (4) \\
\hline
\end{tabular}

$16 \mathrm{~mm} \times 100 \mathrm{~mm}$ Tubes

Peak \#

Start Tube

End Tube

A:3
A:4
A:5
A:6
A:11
A:12
A:13
A:14
A:16
A:18

A:3

A:4

2

3

4

5

6

7

8

9

10

A:5

A:7

A:11

A:12

A:13

A:15

A:17

A:20

\begin{tabular}{|c|c|c|c|}
\hline Duration & \multicolumn{1}{c}{$\%$ B } & \multicolumn{1}{c|}{ Solvent A } & Solvent B \\
\hline $\mathbf{0 . 0}$ & $\mathbf{0 . 0}$ & A2 dichlorometha & B2 methanol \\
$\mathbf{0 . 0}$ & $\mathbf{0 . 0}$ & A2 dichlorometha & B2 methanol \\
11.1 & $\mathbf{7 . 4}$ & A2 dichlorometha & B2 methanol \\
& & & \\
& & & \\
& & & \\
& & & \\
& & & \\
\hline
\end{tabular}



Figure S17. 3-\{6-[5-(3,5-Di-tert-butyl-phenyl)-2H-pyrazol-3-yl]-pyridin-2-yl\}-5,6-diphenyl-[1,2,4]triazine
Sample: VGB-B-211
Rf 200

4x Column: 2

RediSep Column: Silica 4g

Flow Rate: $18 \mathrm{ml} / \mathrm{min}$

Equilibration Volume: $33.6 \mathrm{ml}$

Initial Waste: $0.0 \mathrm{ml}$

Air Purge: $1.0 \mathrm{~min}$

Solvent: $\mathrm{Al}_{\mathrm{CH}} \mathrm{CN}$
Peak Tube Volume: Max.

Non-Peak Tube Volume: Max.

Loading Type: Solid

Wavelength 1 (red): 254nm

Peak Width: 30 sec

Threshold: 0.20 AU

Wavelength 2 (purple): $280 \mathrm{~nm}$
All Wavelength (orange): 200-300nm

Peak Width: 30 sec

Threshold: 0.20 AU

Run Notes:

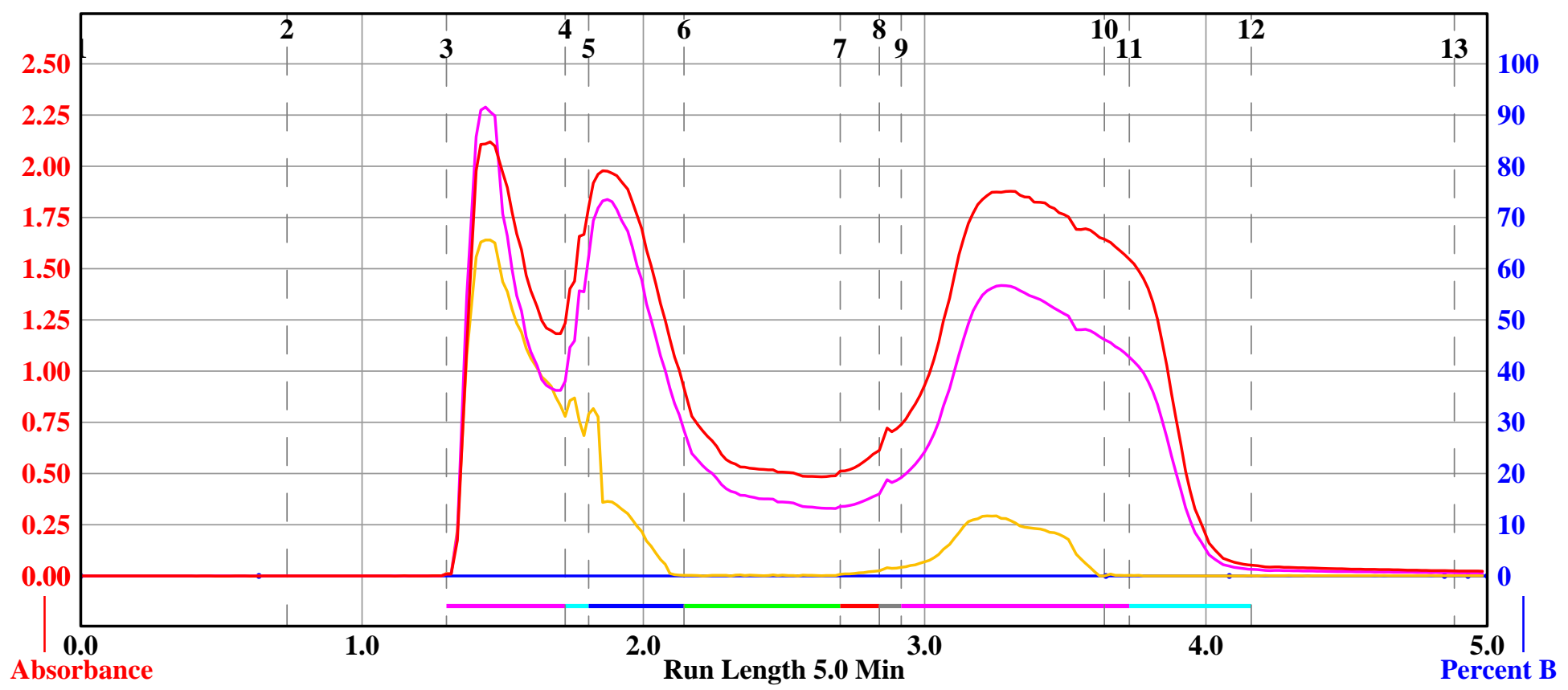

Rack A

Peak

Start Tube

End Tube

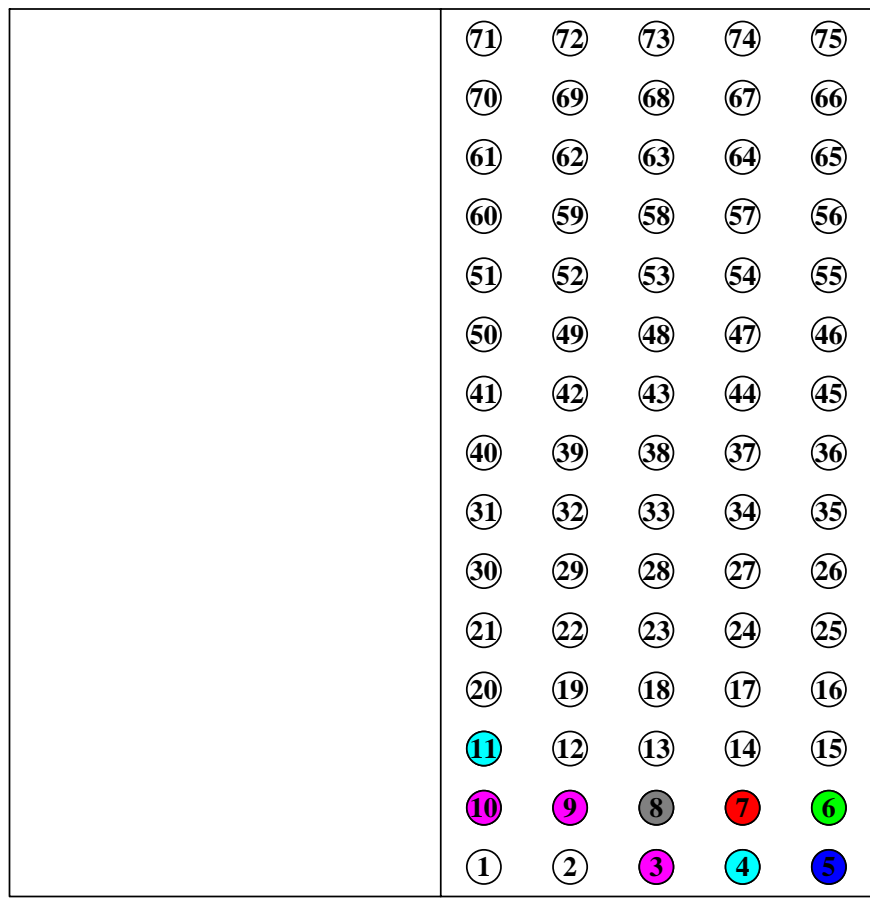

$16 \mathrm{~mm} \times 100 \mathrm{~mm}$ Tubes

\begin{tabular}{|c|c|c|c|}
\hline $\begin{array}{l}1 \\
2 \\
3 \\
4 \\
5 \\
6 \\
7 \\
8\end{array}$ & & $\begin{array}{c}A: 3 \\
A: 4 \\
A: 5 \\
A: 6 \\
A: 7 \\
A: 8 \\
A: 9 \\
A: 11\end{array}$ & $\begin{array}{c}A: 3 \\
A: 4 \\
A: 5 \\
A: 6 \\
A: 7 \\
A: 8 \\
A: 10 \\
A: 11\end{array}$ \\
\hline Duration & $\%$ B & Solvent A & Solvent B \\
\hline $\begin{array}{l}\text { 0.0 } \\
\text { 0.6 } \\
3.0 \\
0.4 \\
\text { 0.8 } \\
\text { 0.1 } \\
\text { 0.1 }\end{array}$ & $\begin{array}{l}\mathbf{0 . 0} \\
\mathbf{0 . 0} \\
\mathbf{0 . 0} \\
\mathbf{0 . 0} \\
\mathbf{0 . 0} \\
\mathbf{0 . 0} \\
\mathbf{0 . 0}\end{array}$ & $\begin{array}{l}\text { A1 hexane } \\
\text { A1 hexane } \\
\text { A1 hexane } \\
\text { A1 hexane } \\
\text { A1 hexane } \\
\text { A1 hexane } \\
\text { A1 hexane }\end{array}$ & $\begin{array}{l}\text { B1 ethyl acetate } \\
\text { B1 ethyl acetate } \\
\text { B1 ethyl acetate } \\
\text { B1 ethyl acetate } \\
\text { B1 ethyl acetate } \\
\text { B1 ethyl acetate } \\
\text { B1 ethyl acetate }\end{array}$ \\
\hline
\end{tabular}


Figure S18. 5,6-Diphenyl-3-[6-(5-pyridin-4-yl-2H-pyrazol-3-yl)-pyridin-2-yl]-[1,2,4]triazine (19) Sample: VGB-B-209

4x Column: 2

RediSep Column: Silica $4 \mathrm{~g}$

Flow Rate: $18 \mathrm{ml} / \mathrm{min}$

Equilibration Volume: $33.6 \mathrm{ml}$

Initial Waste: $0.0 \mathrm{ml}$

Air Purge: $1.0 \mathrm{~min}$

Solvent: $\mathrm{A} 1 \mathrm{CH}_{3} \mathrm{CN}$
Peak Tube Volume: Max.

Non-Peak Tube Volume: Max.

Loading Type: Solid

Wavelength 1 (red): $254 \mathrm{~nm}$

Peak Width: 30 sec

Threshold: $0.20 \mathrm{AU}$

Wavelength 2 (purple): $280 \mathrm{~nm}$
All Wavelength (orange): 200-300nm

Peak Width: 30 sec

Threshold: 0.20 AU

Run Notes:

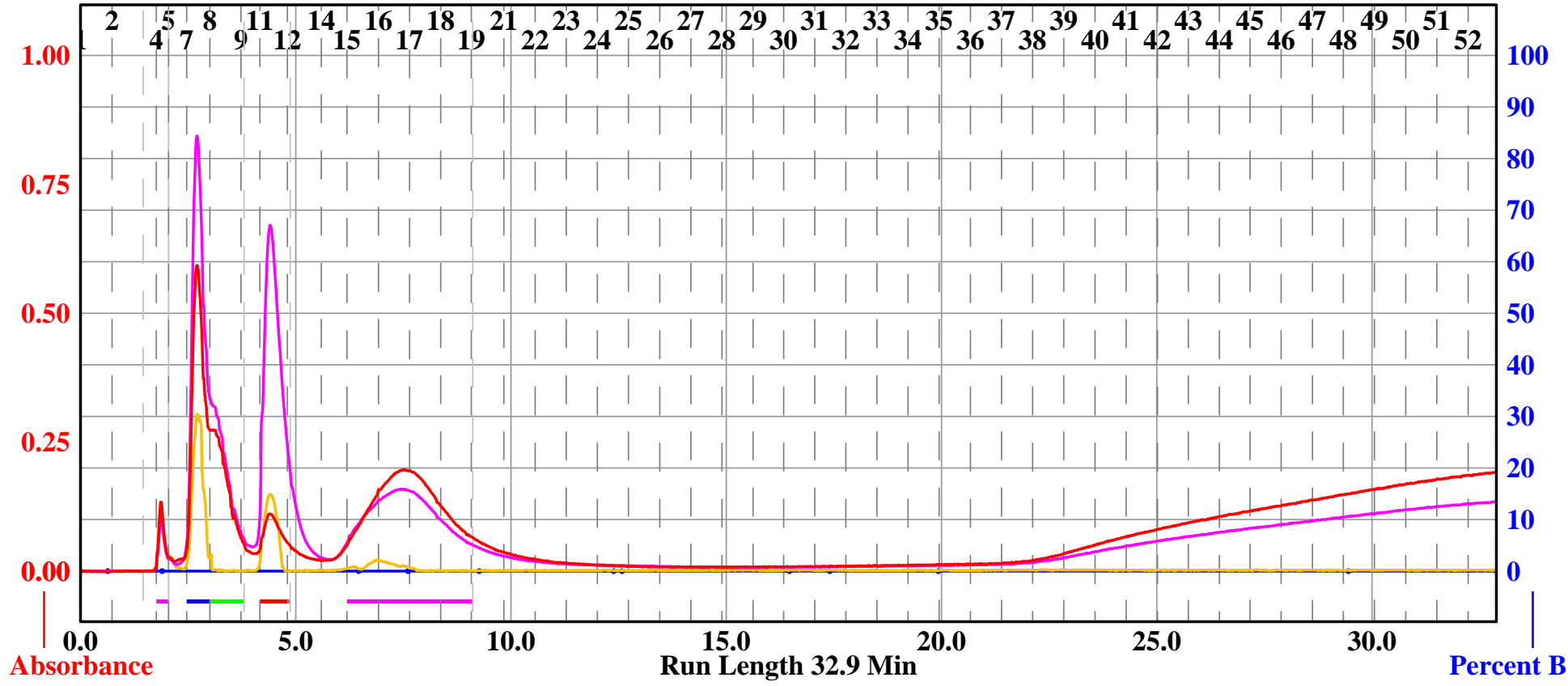

Rack A

\begin{tabular}{|c|c|c|c|}
\hline (71) & (72) & (73) & (14) \\
\hline (70) & (6) & (68) & (67) \\
\hline 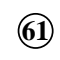 & (62) & (63) & (64) \\
\hline (60) & (59) & (58) & (57) \\
\hline ค & (52) & (33) & (54) \\
\hline 3 & (49) & (48) & (47) \\
\hline & (42) & (43) & (44) \\
\hline (4) & (39) & (38) & (37) \\
\hline (31) & (32) & (33) & (34) \\
\hline 2 & (29) & (28) & (27) \\
\hline & (22) & (23) & (24) \\
\hline & 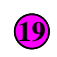 & (18) & (1) \\
\hline & & (13) & (14) \\
\hline & & (8) & \\
\hline & (2) & (3) & (4) \\
\hline
\end{tabular}

$16 \mathrm{~mm} \times 100 \mathrm{~mm}$ Tubes
Run Length 32.9 Min

Peak \#

Start Tube

End Tube

\begin{tabular}{|c|c|c|c|}
\hline $\begin{array}{l}1 \\
2 \\
3 \\
4 \\
5 \\
6 \\
7\end{array}$ & & $\begin{array}{c}A: 4 \\
A: 5 \\
A: 7 \\
A: 8 \\
A: 11 \\
A: 12 \\
A: 15\end{array}$ & $\begin{array}{c}A: 4 \\
A: 5 \\
A: 7 \\
A: 9 \\
A: 11 \\
A: 12 \\
A: 19\end{array}$ \\
\hline Duration & $\% \mathbf{B}$ & Solvent A & Solvent B \\
\hline 0.0 & 0.0 & A1 hexane & B1 ethyl acetate \\
\hline 0.6 & 0.0 & A1 hexane & B1 ethyl acetate \\
\hline 1.3 & 0.0 & A1 hexane & B1 ethyl acetate \\
\hline 4.6 & 0.0 & A1 hexane & B1 ethyl acetate \\
\hline 1.1 & 0.0 & A1 hexane & B1 ethyl acetate \\
\hline 1.7 & 0.0 & A1 hexane & B1 ethyl acetate \\
\hline 3.1 & 0.0 & A1 hexane & B1 ethyl acetate \\
\hline 0.2 & $\mathbf{0 . 0}$ & A1 hexane & B1 ethyl acetate \\
\hline 3.9 & 0.0 & A1 hexane & B1 ethyl acetate \\
\hline 0.9 & 0.0 & A1 hexane & B1 ethyl acetate \\
\hline$\cdots$ & $\cdots$ & ... & $\cdots$ \\
\hline
\end{tabular}


Figure S19. 5,6-Bis-(4-cyclopropyl-phenyl)-3-[6-(5-phenyl-2H-pyrazol-3-yl)-pyridin-2-yl]-[1,2,4]triazine (20) Sample: VGB-B-265

4x Column: 2

RediSep Column: Silica 4g

Flow Rate: $18 \mathrm{ml} / \mathrm{min}$

Equilibration Volume: $33.6 \mathrm{ml}$

Initial Waste: $0.0 \mathrm{ml}$

Air Purge: 0.5 min

Solvent: $\mathrm{A} 1 \mathrm{CH}_{3} \mathrm{CN}$
Peak Tube Volume: Max.

Non-Peak Tube Volume: Max.

Loading Type: Solid

Wavelength 1 (red): 254nm

Peak Width: $30 \mathrm{sec}$

Threshold: 0.20 AU

Wavelength 2 (purple): $280 \mathrm{~nm}$
Monday 28 January 2019 07:26PM

All Wavelength (orange): 200-300nm Peak Width: 30 sec

Threshold: 0.20 AU

Run Notes:

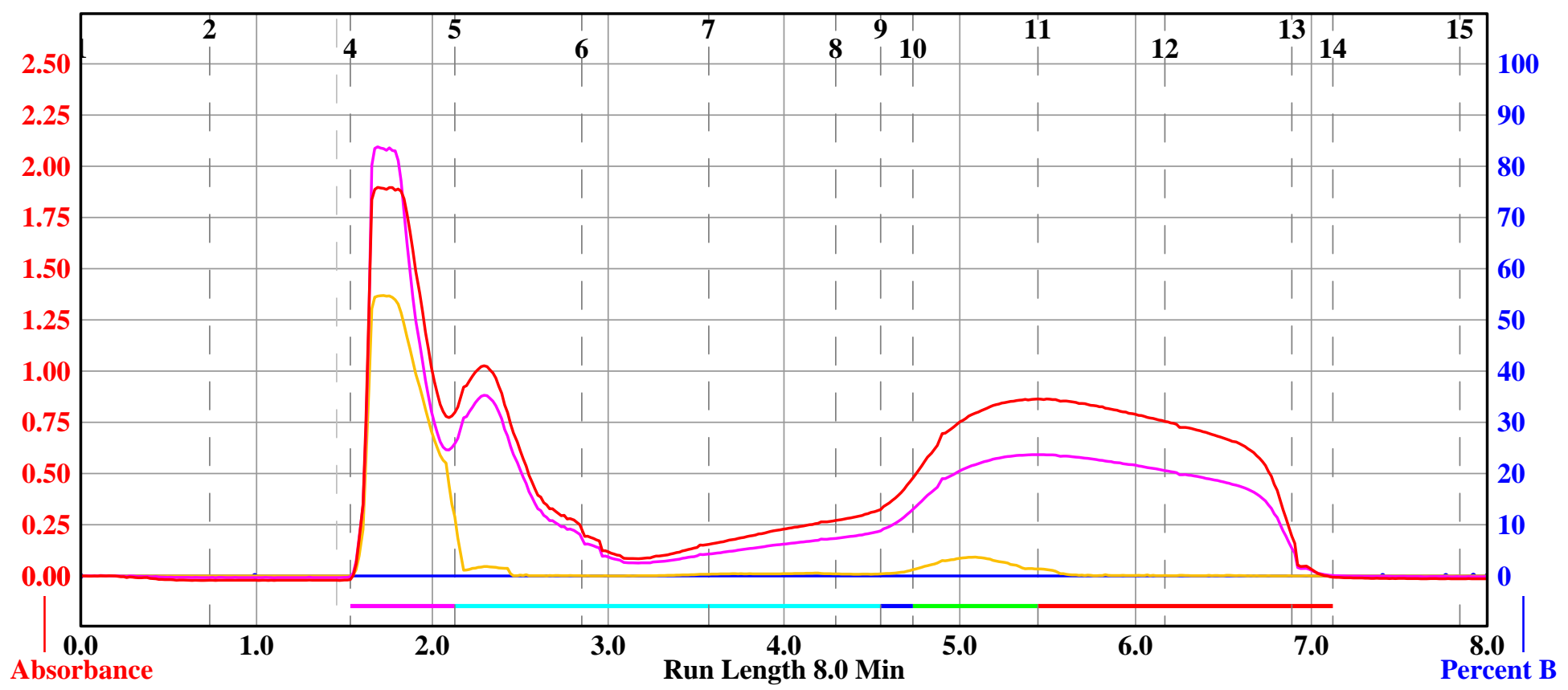

Rack A

\begin{tabular}{|c|c|c|c|}
\hline (71) & (72) & (13) & (74) \\
\hline (70) & (69) & (68) & (67) \\
\hline (61) & (62) & (63) & (64) \\
\hline (60) & (59) & (58) & (57) \\
\hline (51) & (52) & (53) & (54) \\
\hline 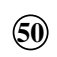 & (49) & (48) & (47) \\
\hline (41) & (42) & (43) & (44) \\
\hline$(\pi)$ & (39) & (38) & (37) \\
\hline (31) & (32) & (33) & (34) \\
\hline (30) & (29) & (28) & (27) \\
\hline ร & (22) & (23) & (24) \\
\hline (20) & (19) & (18) & (17) \\
\hline (11) & & (13) & (14) \\
\hline (10) & & 8 & (7) \\
\hline (1) & (2) & (3) & (4) \\
\hline
\end{tabular}

$16 \mathrm{~mm} \times 100 \mathrm{~mm}$ Tubes

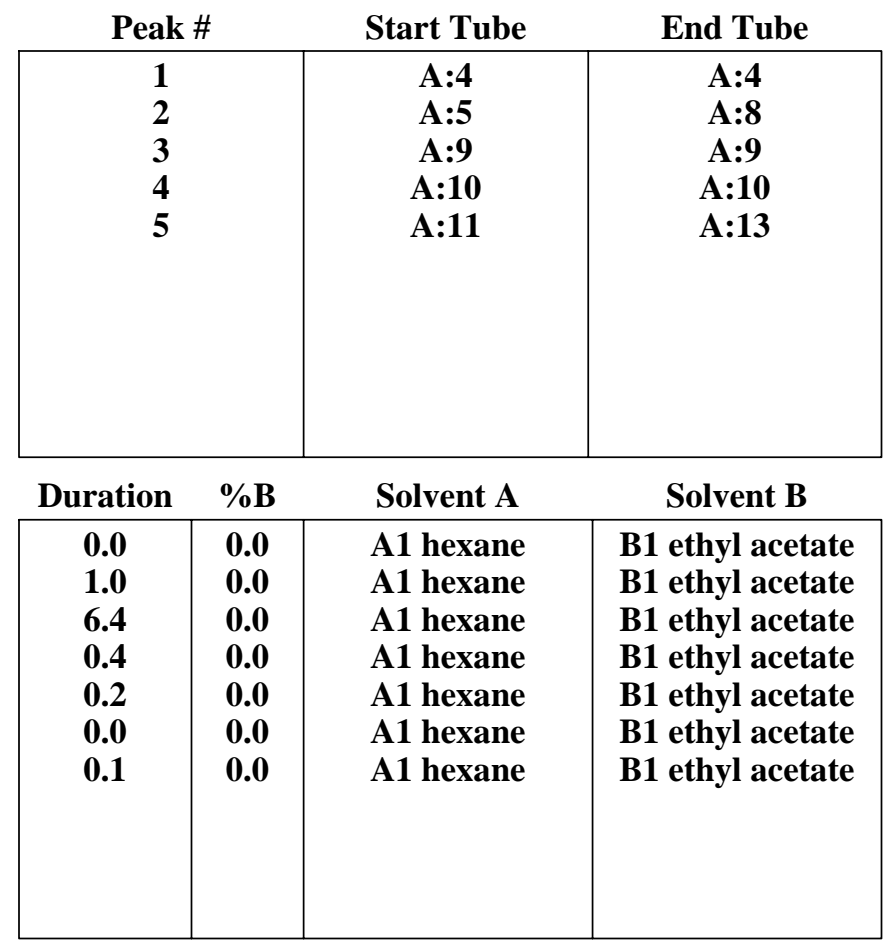


Figure S20. 5,6-Bis-(4-cyclopropyl-phenyl)-3-\{6-[5-(2-iodo-phenyl)-2H-pyrazol-3-y|]-pyridin-2-y|\}-triazine (21) Sample: VGB-B-269

Rf 200

4x Column: 2

RediSep Column: Silica $4 \mathrm{~g}$

Flow Rate: $18 \mathrm{ml} / \mathrm{min}$

Equilibration Volume: $33.6 \mathrm{ml}$

Initial Waste: $0.0 \mathrm{ml}$

Air Purge: $0.5 \mathrm{~min}$

Solvent: $\mathrm{A} 1 \mathrm{CH}_{3} \mathrm{CN}$
Peak Tube Volume: Max.

Non-Peak Tube Volume: Max.

Loading Type: Solid

Wavelength 1 (red): 254nm

Peak Width: 30 sec

Threshold: $0.20 \mathrm{AU}$

Wavelength 2 (purple): $280 \mathrm{~nm}$
Monday 28 January 2019 02:11PM

All Wavelength (orange): 200-300nm

Peak Width: 30 sec

Threshold: 0.20 AU

Run Notes:

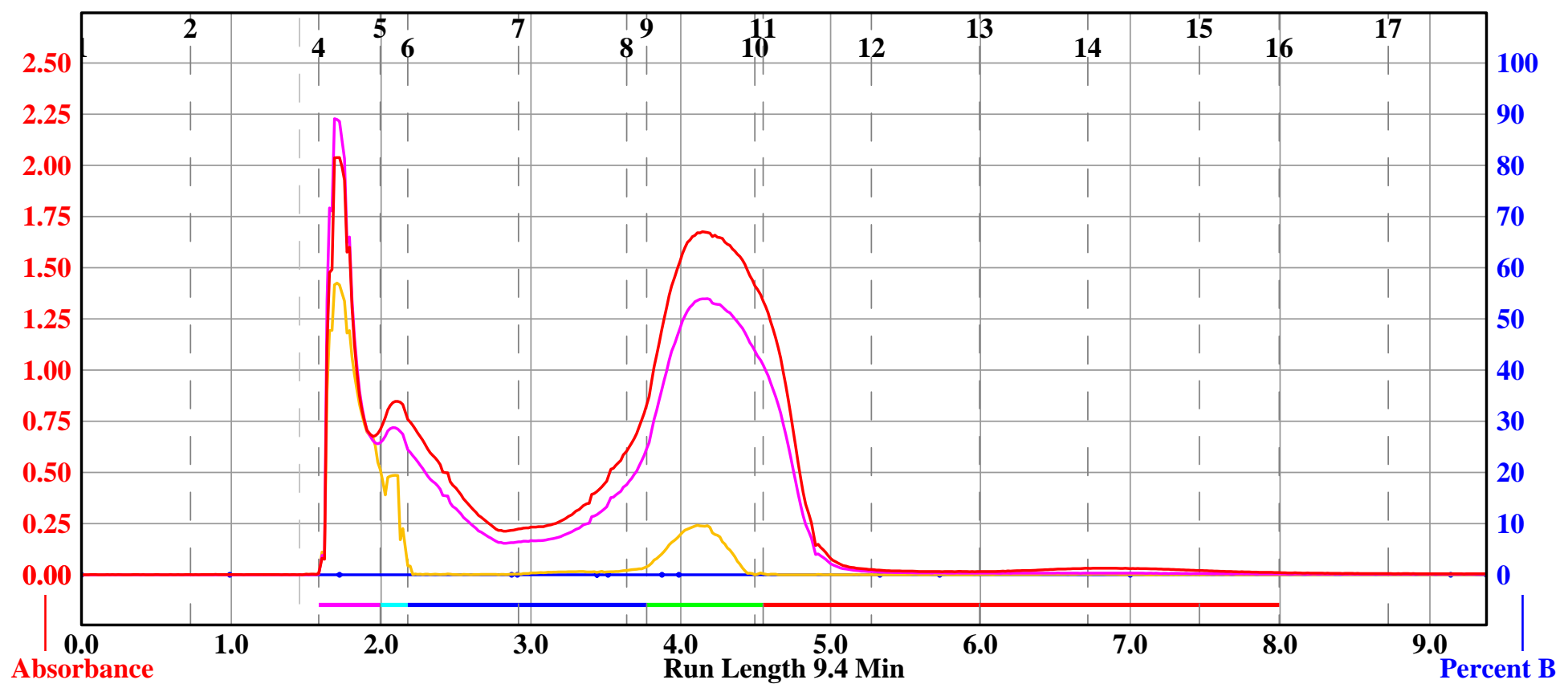

Rack A

\begin{tabular}{|c|c|c|c|}
\hline (71) & (72) & (13) & (14) \\
\hline (1) & (6) & (68) & (67) \\
\hline & (62) & (63) & (64) \\
\hline (60) & (99) & (58) & (57) \\
\hline 51 & (52) & (33) & (54) \\
\hline (30) & (49) & (48) & (47) \\
\hline & (42) & (43) & (44) \\
\hline & (39) & (38) & (37) \\
\hline (31) & (32) & (33) & (34) \\
\hline & (29) & (28) & (27) \\
\hline & (22) & (23) & (24) \\
\hline & (19) & (18) & (17) \\
\hline & (12) & & (1) \\
\hline & (9) & & \\
\hline & (2) & (3) & (4) \\
\hline
\end{tabular}

$16 \mathrm{~mm} \times 100 \mathrm{~mm}$ Tubes

\begin{tabular}{|c|c|c|c|}
\hline \multicolumn{2}{|c|}{ Peak \# } & Start Tube & End Tube \\
\hline $\begin{array}{l}\mathbf{1} \\
\mathbf{2} \\
\mathbf{3} \\
\mathbf{4} \\
\mathbf{5}\end{array}$ & & $\begin{array}{c}A: 4 \\
A: 5 \\
A: 6 \\
A: 9 \\
A: 11\end{array}$ & $\begin{array}{c}A: 4 \\
A: 5 \\
A: 8 \\
A: 10 \\
A: 15\end{array}$ \\
\hline Duration & $\%$ B & Solvent A & Solvent B \\
\hline 0.0 & 0.0 & A1 hexane & B1 ethyl acetate \\
\hline 1.0 & 0.0 & A1 hexane & B1 ethyl acetate \\
\hline 0.7 & 0.0 & A1 hexane & B1 ethyl acetate \\
\hline 1.1 & 0.0 & A1 hexane & B1 ethyl acetate \\
\hline 0.0 & $\mathbf{0 . 0}$ & A1 hexane & B1 ethyl acetate \\
\hline 0.5 & 0.0 & A1 hexane & B1 ethyl acetate \\
\hline 0.0 & 0.0 & A1 hexane & B1 ethyl acetate \\
\hline 0.1 & 0.0 & A1 hexane & B1 ethyl acetate \\
\hline 0.4 & 0.0 & A1 hexane & B1 ethyl acetate \\
\hline 0.1 & 0.0 & A1 hexane & B1 ethyl acetate \\
\hline$\cdots$ & $\cdots$ & $\ldots$ & $\ldots$ \\
\hline
\end{tabular}


Figure S21. 3-\{6-[5-(4-Bromo-phenyl)-2H-pyrazol-3-yl]-pyridin-2-yl\}-5,6-bis-(4-butyl-phenyl)-triazine (22) Sample: VGB-B-221

4x Column: 2

RediSep Column: Silica 4g

Flow Rate: $18 \mathrm{ml} / \mathrm{min}$

Equilibration Volume: $33.6 \mathrm{ml}$

Initial Waste: $0.0 \mathrm{ml}$

Air Purge: $1.0 \mathrm{~min}$

Solvent: $\mathrm{A} 1 \mathrm{CH}_{3} \mathrm{CN}$
Peak Tube Volume: Max.

Non-Peak Tube Volume: Max.

Loading Type: Solid

Wavelength 1 (red): 254nm

Peak Width: 30 sec

Threshold: 0.20 AU

Wavelength 2 (purple): 280nm
Tuesday 15 January 2019 04:55PM

All Wavelength (orange): 200-300nm

Peak Width: $30 \mathrm{sec}$

Threshold: 0.20 AU

Run Notes:

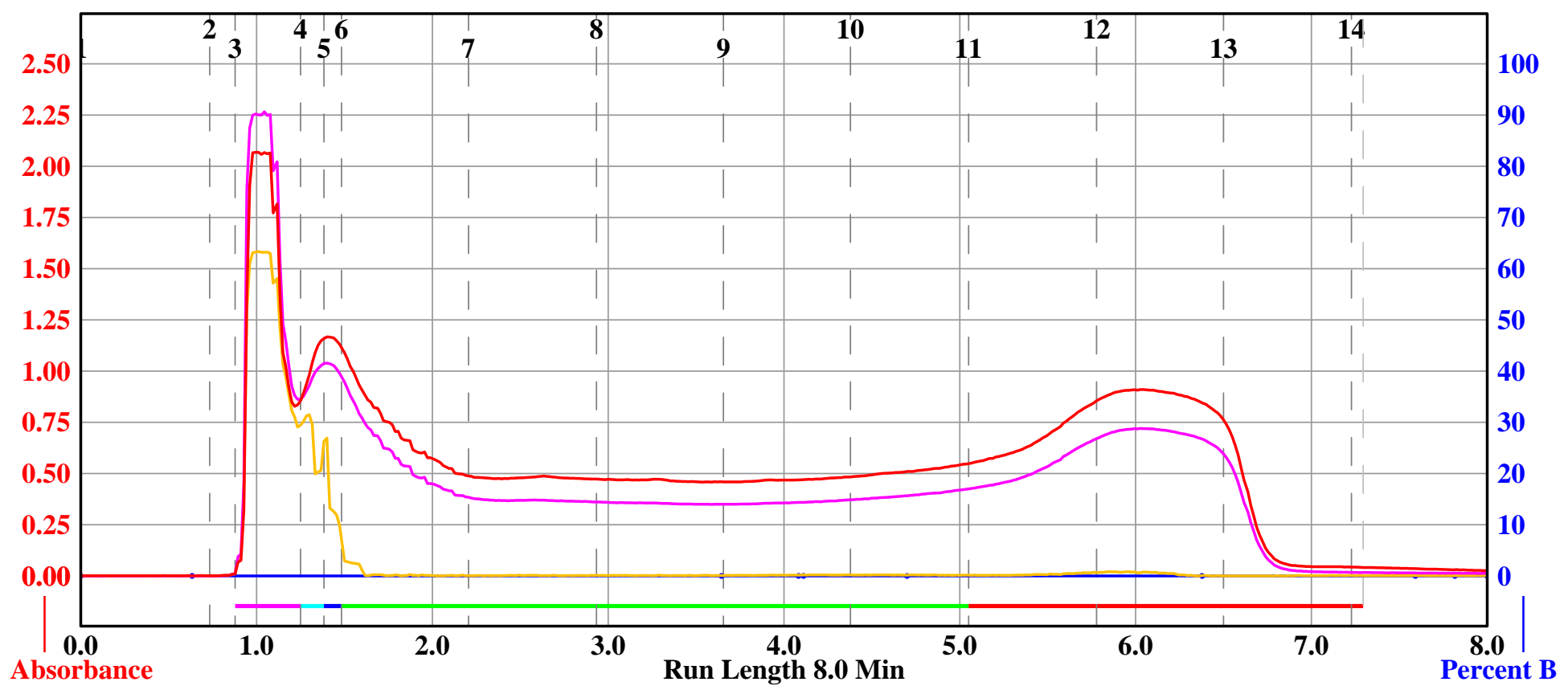

Rack A

\begin{tabular}{|c|c|c|c|c|}
\hline (71) & (72) & (13) & (74) & (75) \\
\hline (70) & (69) & (68) & (67) & (66) \\
\hline (61) & (62) & (63) & (64) & (65) \\
\hline (60) & (59) & (58) & (57) & 56 \\
\hline (51) & (52) & (33) & (54) & (55) \\
\hline (50) & (49) & (48) & (47) & (46) \\
\hline (41) & (42) & (43) & (44) & (45) \\
\hline (40) & (39) & (38) & (37) & (36) \\
\hline (31) & (32) & (33) & (34) & (35) \\
\hline (30) & (29) & (28) & (27) & (26) \\
\hline (21) & (22) & (23) & (24) & (25) \\
\hline (20) & (19) & (18) & (17) & (16) \\
\hline (11) & (12) & (13) & (14) & (15) \\
\hline (10) & (9) & (8) & (7) & (6) \\
\hline (1) & (2) & (3) & (4) & \\
\hline
\end{tabular}

$16 \mathrm{~mm} \times 100 \mathrm{~mm}$ Tubes

\begin{tabular}{|c|c|c|c|}
\hline \multicolumn{2}{|c|}{ Peak \# } & Start Tube & End Tube \\
\hline \multicolumn{2}{|c|}{$\begin{array}{l}1 \\
2 \\
3 \\
4 \\
5\end{array}$} & $\begin{array}{c}\text { A:3 } \\
\text { A:4 } \\
\text { A:5 } \\
\text { A:6 } \\
\text { A:11 }\end{array}$ & $\begin{array}{c}\text { A:3 } \\
\text { A:4 } \\
\text { A:5 } \\
\text { A:10 } \\
\text { A:14 }\end{array}$ \\
\hline Duration & $\% \mathrm{~B}$ & Solvent A & Solvent B \\
\hline 0.0 & 0.0 & A1 hexane & B1 ethyl acetate \\
\hline 0.6 & 0.0 & A1 hexane & B1 ethyl acetate \\
\hline 3.0 & $\mathbf{0 . 0}$ & A1 hexane & B1 ethyl acetate \\
\hline 0.4 & 0.0 & A1 hexane & B1 ethyl acetate \\
\hline 0.0 & 0.0 & A1 hexane & B1 ethyl acetate \\
\hline 0.6 & 0.0 & A1 hexane & B1 ethyl acetate \\
\hline 1.7 & 0.0 & A1 hexane & B1 ethyl acetate \\
\hline 1.2 & 0.0 & A1 hexane & B1 ethyl acetate \\
\hline 0.2 & 0.0 & A1 hexane & B1 ethyl acetate \\
\hline 0.2 & 0.0 & A1 hexane & B1 ethyl acetate \\
\hline
\end{tabular}


Figure S22. 3-\{6-[5-(4-Bromo-phenyl)-2H-pyrazol-3-yl]-pyridin-2-yl\}-5,6-bis-[4-(3,3-dimethyl-butyl)-phenyl]\{ł,2,4]triazine

4x Column: 2

RediSep Column: Silica 4g

Flow Rate: $18 \mathrm{ml} / \mathrm{min}$

Equilibration Volume: $33.6 \mathrm{ml}$

Initial Waste: $0.0 \mathrm{ml}$

Air Purge: $2.3 \mathrm{~min}$

Solvent: A2 dichloromethane

Solvent: B2 2-propanol

\section{Rf 200}

Peak Tube Volume: Max.

Non-Peak Tube Volume: Max.

Loading Type: Solid

Wavelength 1 (red): 254nm

Peak Width: $30 \mathrm{sec}$

Threshold: 0.20 AU

Wavelength 2 (purple): $280 \mathrm{~nm}$
Thursday 29 November 2018 07:32AM

All Wavelength (orange): $200-300 \mathrm{~nm}$

Peak Width: 30 sec

Threshold: 0.20 AU

Run Notes:

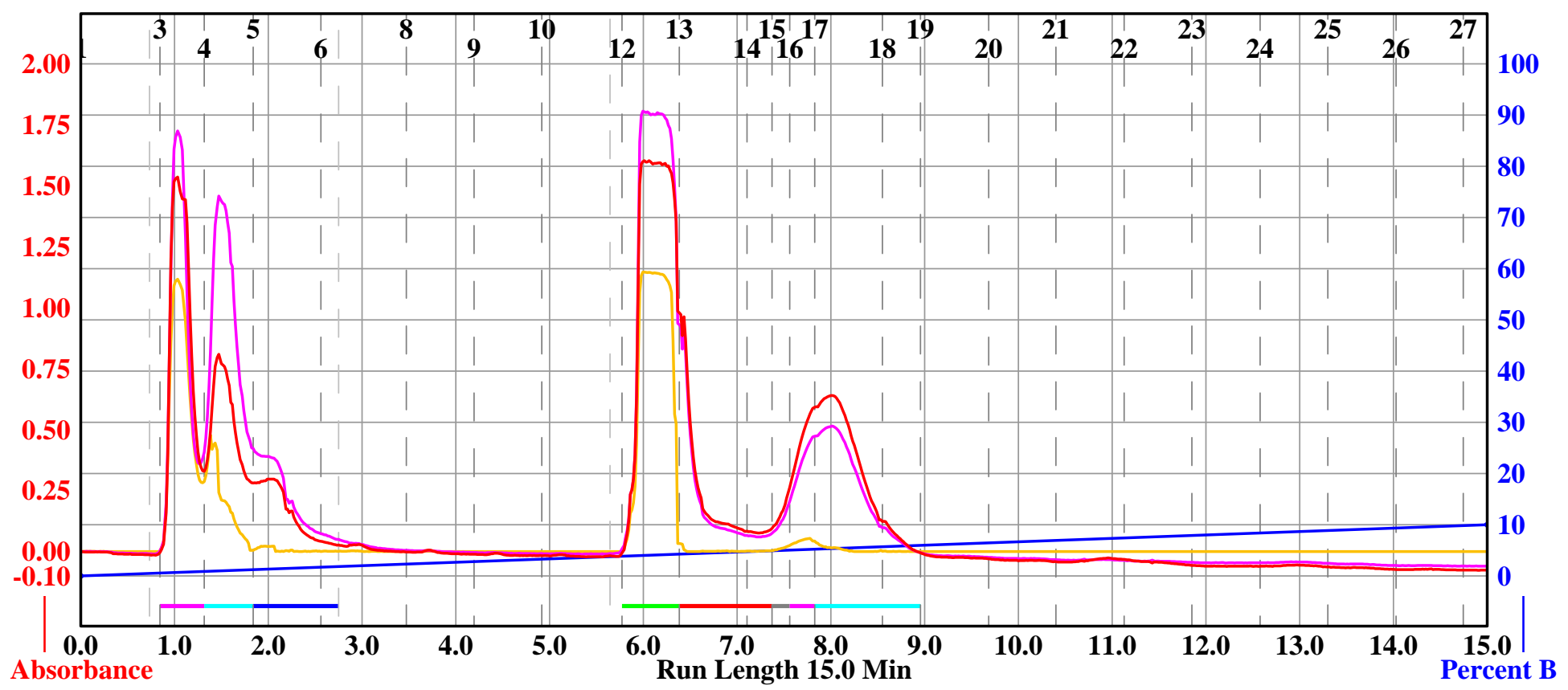

Rack A

\begin{tabular}{|c|c|c|c|c|}
\hline (71) & (72) & (73) & (74) & (75) \\
\hline (70) & (69) & 68 & (67) & (66) \\
\hline (61) & (62) & (63) & (64) & (A) \\
\hline (60) & (59) & (58) & (57) & \\
\hline (51) & (52) & (33) & (54) & \\
\hline (50) & (49) & (48) & (47) & \\
\hline (41) & (42) & (43) & (44) & \\
\hline (40) & (39) & (38) & (37) & \\
\hline (121) & (32) & (33) & (34) & \\
\hline (30) & (29) & (28) & (27) & \\
\hline (1) & (22) & (23) & (24) & \\
\hline (20) & (19) & (18) & (17) & \\
\hline (11) & (12) & (13) & (14) & \\
\hline (10) & (9) & (8) & (7) & \\
\hline (1) & (2) & (3) & (4) & \\
\hline
\end{tabular}

$16 \mathrm{~mm} \times 100 \mathrm{~mm}$ Tubes

\begin{tabular}{|c|c|c|c|}
\hline \multicolumn{2}{|c|}{ Peak \# } & Start Tube & End Tube \\
\hline $\begin{array}{l}1 \\
2 \\
3 \\
4 \\
5 \\
6 \\
7 \\
8\end{array}$ & & $\begin{array}{l}\text { A:3 } \\
\text { A:4 } \\
\text { A:5 } \\
\text { A:12 } \\
\text { A:13 } \\
\text { A:15 } \\
\text { A:16 } \\
\text { A:17 }\end{array}$ & $\begin{array}{l}\text { A:3 } \\
\text { A:4 } \\
\text { A:6 } \\
\text { A:12 } \\
\text { A:14 } \\
\text { A:15 } \\
\text { A:16 } \\
\text { A:18 }\end{array}$ \\
\hline Duration & $\%$ B & Solvent A & Solvent B \\
\hline $\begin{array}{c}0.0 \\
15.0\end{array}$ & $\begin{array}{c}0.0 \\
10.0\end{array}$ & $\begin{array}{l}\text { A2 dichlorometha } \\
\text { A2 dichlorometha }\end{array}$ & $\begin{array}{l}\text { B2 methanol } \\
\text { B2 methanol }\end{array}$ \\
\hline
\end{tabular}


Figure S23. 3-\{6-[5-(4-tert-Butyl-phenyl)-2H-pyrazol-3-yl]-pyridin-2-yl\}-5,6-bis-[4-(3,3-dimethyl-butyl)-

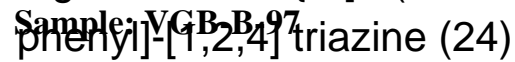

4x Column: 2

RediSep Column: Silica 4g

Flow Rate: $18 \mathrm{ml} / \mathrm{min}$

Equilibration Volume: $33.6 \mathrm{ml}$

Initial Waste: $0.0 \mathrm{ml}$

Air Purge: 2.5 min

Solvent: A2 dichloromethane

Solvent: B2 2-propanol

\section{Rf 200}

Peak Tube Volume: Max.

Non-Peak Tube Volume: Max.

Loading Type: Solid

Wavelength 1 (red): 254nm

Peak Width: $30 \mathrm{sec}$

Threshold: 0.20 AU

Wavelength 2 (purple): $280 \mathrm{~nm}$
Tuesday 27 November 2018 10:47AM

All Wavelength (orange): 200-300nm

Peak Width: 30 sec

Threshold: 0.20 AU

Run Notes:

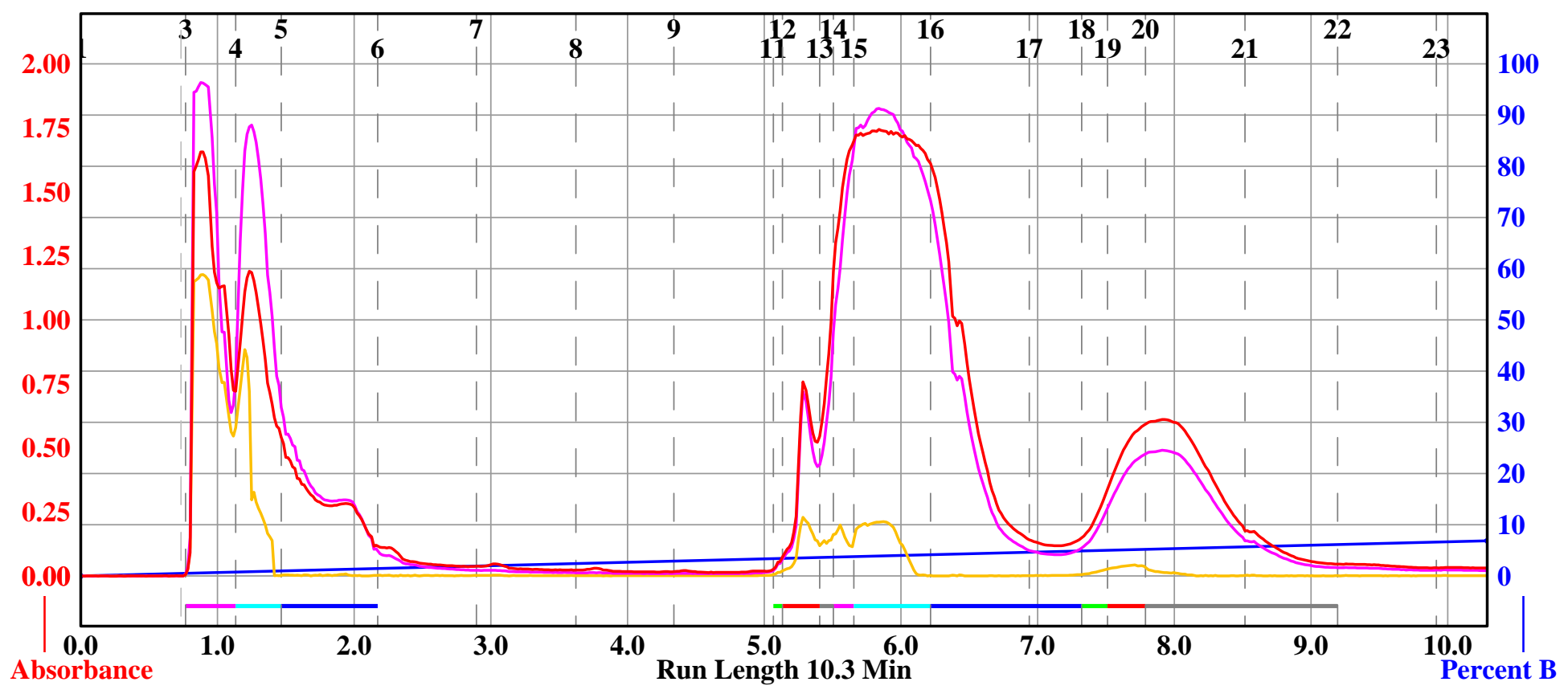

Rack A

\begin{tabular}{|c|c|c|c|c|}
\hline (11) & (12) & (13) & (14) & (75) \\
\hline (70) & (6) & (68) & (67) & 6 \\
\hline (11) & (12) & (3) & (64) & (65) \\
\hline (60) & (59) & (58) & (57) & 56 \\
\hline (11) & (52) & (53) & (54) & 53 \\
\hline (50) & (49) & (48) & (47) & (46 \\
\hline (41) & (42) & (43) & (44) & (45) \\
\hline (40) & (39) & (38) & (37) & 36 \\
\hline (31) & (32) & (33) & (34) & (35) \\
\hline (30) & (29) & (28) & (27) & (26) \\
\hline (21) & (22) & (23) & (24) & (25) \\
\hline (20) & (19) & (18) & (1) & 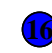 \\
\hline (11) & (12) & (13) & (14) & i \\
\hline (10) & (9) & (8) & (7) & (6) \\
\hline (1) & (2) & (3) & (4) & \\
\hline
\end{tabular}

$16 \mathrm{~mm} \times 100 \mathrm{~mm}$ Tubes

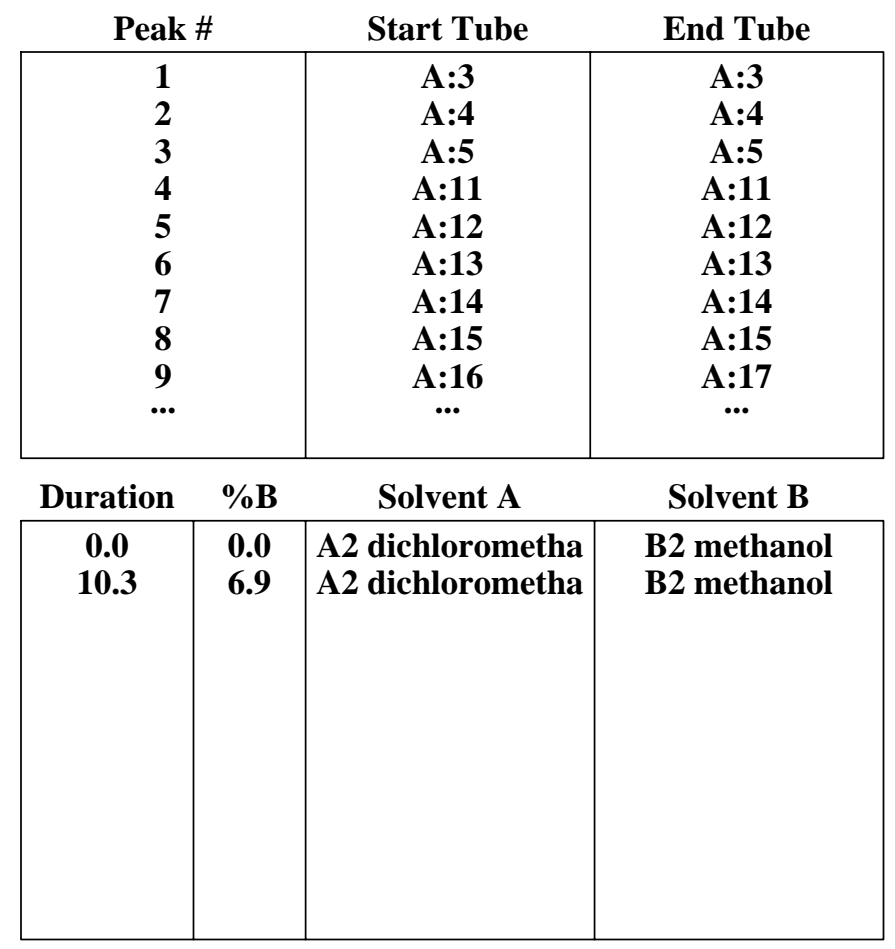


Figure S24. 5,6-Bis-(4-butyl-phenyl)-3-\{6-[5-(3,5-di-tert-butyl-phenyl)-2H-pyrazol-3-yl]-pyridin-2-yl\}-

$\{1,2,4$ ]triazine 2 (25)

4x Column: 2

RediSep Column: Silica 4g

Flow Rate: $18 \mathrm{ml} / \mathrm{min}$

Equilibration Volume: $33.6 \mathrm{ml}$

Initial Waste: $0.0 \mathrm{ml}$

Air Purge: $1.0 \mathrm{~min}$

Solvent: $\mathrm{A} 1 \mathrm{CH}_{3} \mathrm{CN}$

\section{Rf 200}

Peak Tube Volume: Max.

Non-Peak Tube Volume: Max.

Loading Type: Solid

Wavelength 1 (red): 254nm

Peak Width: $30 \mathrm{sec}$

Threshold: 0.20 AU

Wavelength 2 (purple): $280 \mathrm{~nm}$
Wednesday 16 January 2019 03:11PM

All Wavelength (orange): 200-300nm Peak Width: 30 sec

Threshold: 0.20 AU

Run Notes:

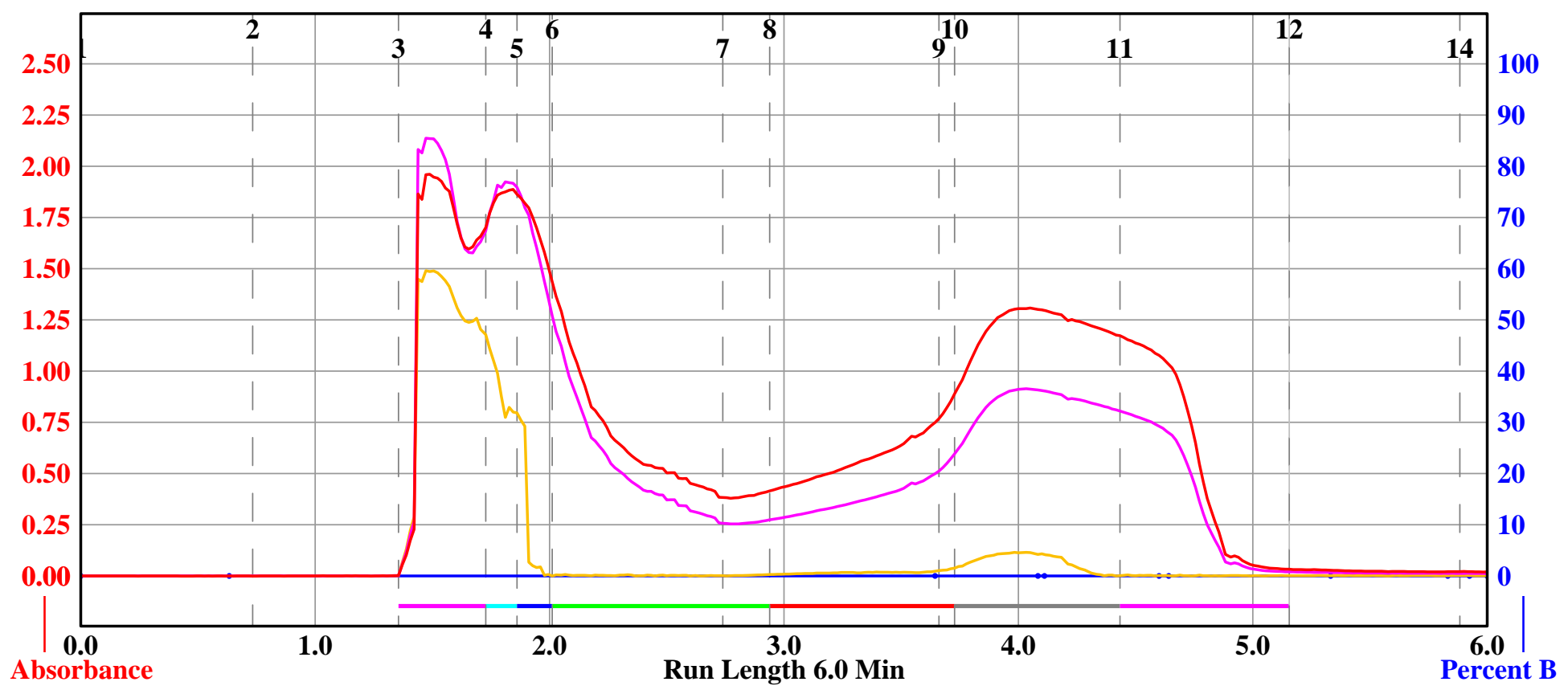

Rack A

\begin{tabular}{|c|c|c|c|}
\hline (71) & (72) & (73) & (74) \\
\hline (70) & (69) & (68) & (67) \\
\hline (61) & (62) & (63) & (64) \\
\hline (6) & (59) & (58) & (57) \\
\hline (51) & (52) & (53) & (54) \\
\hline (7) & (49) & (48) & (47) \\
\hline (41) & (42) & (43) & (44) \\
\hline (40) & (39) & (38) & (37) \\
\hline (31) & (32) & (33) & (34) \\
\hline (30) & (29) & (28) & (27) \\
\hline (21) & (22) & (23) & (24) \\
\hline (20) & (19) & (18) & (17) \\
\hline 111) & (12) & (13) & (14) \\
\hline 12 & (9) & (8) & (7) \\
\hline (1) & (2) & (3) & (4) \\
\hline
\end{tabular}

$16 \mathrm{~mm} \times 100 \mathrm{~mm}$ Tubes

\begin{tabular}{|c|c|c|c|}
\hline \multicolumn{2}{|c|}{ Peak \# } & Start Tube & End Tube \\
\hline $\begin{array}{l}1 \\
2 \\
3 \\
4 \\
5 \\
6 \\
7\end{array}$ & & $\begin{array}{l}A: 3 \\
A: 4 \\
A: 5 \\
A: 6 \\
A: 8 \\
A: 10 \\
A: 11\end{array}$ & $\begin{array}{c}A: 3 \\
A: 4 \\
A: 5 \\
A: 7 \\
A: 9 \\
A: 10 \\
A: 12\end{array}$ \\
\hline Duration & $\% \mathbf{B}$ & Solvent A & Solvent B \\
\hline 0.0 & 0.0 & A1 hexane & B1 ethyl acetate \\
\hline 0.6 & 0.0 & A1 hexane & B1 ethyl acetate \\
\hline 3.0 & $\mathbf{0 . 0}$ & A1 hexane & B1 ethyl acetate \\
\hline 0.4 & $\mathbf{0 . 0}$ & A1 hexane & B1 ethyl acetate \\
\hline 0.0 & $\mathbf{0 . 0}$ & A1 hexane & B1 ethyl acetate \\
\hline 0.5 & $\mathbf{0 . 0}$ & A1 hexane & B1 ethyl acetate \\
\hline 0.0 & $\mathbf{0 . 0}$ & A1 hexane & B1 ethyl acetate \\
\hline 0.7 & $\mathbf{0 . 0}$ & A1 hexane & B1 ethyl acetate \\
\hline 0.5 & $\mathbf{0 . 0}$ & A1 hexane & B1 ethyl acetate \\
\hline 0.1 & 0.0 & A1 hexane & B1 ethyl acetate \\
\hline 0.1 & $\mathbf{0 . 0}$ & A1 hexane & B1 ethyl acetate \\
\hline
\end{tabular}


Figure S25. 3-\{6-[5-(4-Bromo-phenyl)-2H-pyrazol-3-yl]-pyridin-2-yl\}-5,6-di-p-tolyl-[1,2,4]triazine (26) Sample: VGB-B-107

4x Column: 2

RediSep Column: Silica 4g

Flow Rate: $18 \mathrm{ml} / \mathrm{min}$

Equilibration Volume: $33.6 \mathrm{ml}$

Initial Waste: $0.0 \mathrm{ml}$

Air Purge: $0.5 \mathrm{~min}$

Solvent: A2 dichloromethane

Solvent: B2 2-propanol
Peak Tube Volume: Max.

Non-Peak Tube Volume: Max.

Loading Type: Solid

Wavelength 1 (red): 254nm

Peak Width: 30 sec

Threshold: $0.20 \mathrm{AU}$

Wavelength 2 (purple): 280nm
All Wavelength (orange): 200-300nm

Peak Width: 30 sec

Threshold: 0.20 AU

Run Notes:

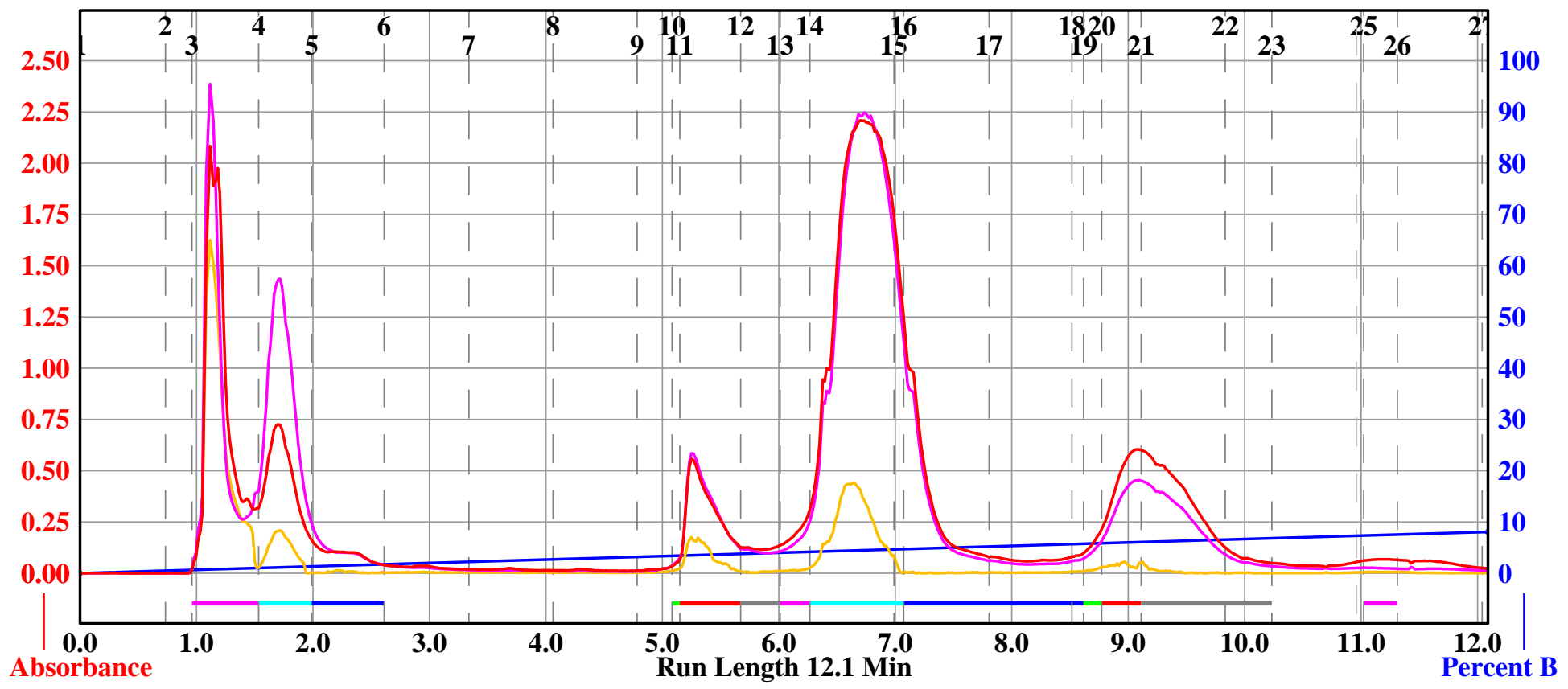

Rack A

\begin{tabular}{|c|c|c|c|}
\hline (71) & (72) & (73) & (74) \\
\hline (70) & (69) & (68) & (67) \\
\hline (61) & (62) & (63) & (64) \\
\hline (60) & (59) & (58) & (57) \\
\hline (51) & (52) & (53) & (54) \\
\hline 5 & (49) & (48) & (47) \\
\hline ) & (42) & (43) & (44) \\
\hline (4) & (39) & (38) & (37) \\
\hline 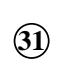 & (32) & (33) & (34) \\
\hline 3 & (29) & (28) & (27) \\
\hline & (22) & (23) & (24) \\
\hline & (19) & & \\
\hline & (12) & (13) & (14) \\
\hline 1 & (9) & (8) & (7) \\
\hline & (2) & (3) & (4) \\
\hline
\end{tabular}

$16 \mathrm{~mm} \times 100 \mathrm{~mm}$ Tubes

Peak \#

Start Tube

End Tube

\section{A:3}

A:4

A:5

A:10

A:11

A:12

A:13

A:14

A:16

...

\begin{tabular}{|c|c|c|c|}
\hline \multicolumn{1}{|c|}{ Duration } & \multicolumn{1}{c}{ \% B } & \multicolumn{1}{c|}{ Solvent A } & Solvent B \\
\hline $\mathbf{0 . 0}$ & $\mathbf{0 . 0}$ & A2 dichlorometha & B2 methanol \\
$\mathbf{1 2 . 1}$ & $\mathbf{8 . 1}$ & A2 dichlorometha & B2 methanol \\
& & & \\
& & & \\
& & & \\
& & & \\
& & & \\
\hline
\end{tabular}


Figure S26. 5,6-Bis-(4-fluoro-phenyl)-3-\{6-[5-(4-methoxy-phenyl)-2H-pyrazol-3-yl]-pyridin-2-yl\}-[1,2,4]triazine (29mp)ple: VGB-B-93

4x Column: 2

RediSep Column: Silica 4g

Flow Rate: $18 \mathrm{ml} / \mathrm{min}$

Equilibration Volume: $33.6 \mathrm{ml}$

Initial Waste: $0.0 \mathrm{ml}$

Air Purge: 2.5 min

Solvent: A2 dichloromethane

Solvent: B2 2-propanol

\section{Rf 200}

Peak Tube Volume: Max.

Non-Peak Tube Volume: Max.

Loading Type: Solid

Wavelength 1 (red): 254nm

Peak Width: $30 \mathrm{sec}$

Threshold: 0.20 AU

Wavelength 2 (purple): $280 \mathrm{~nm}$
Wednesday 28 November 2018 08:21AM

All Wavelength (orange): 200-300nm

Peak Width: 30 sec

Threshold: 0.20 AU

Run Notes:

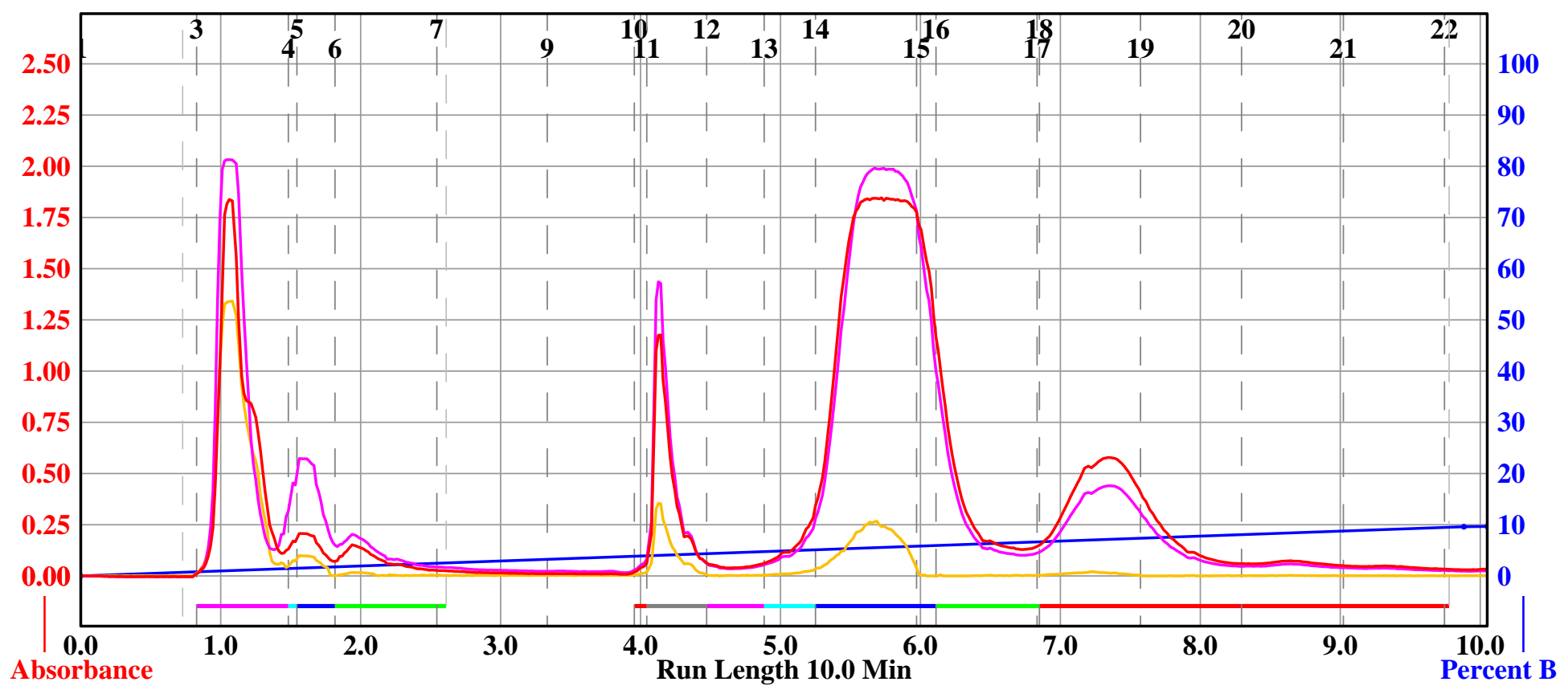

Rack A

\begin{tabular}{|c|c|c|c|c|}
\hline (71) & (12) & (13) & (44) & (75) \\
\hline (70) & (6) & (8) & (67) & (6) \\
\hline (11) & (62) & (63) & (64) & (65) \\
\hline (10) & (59) & (58) & (57) & (56) \\
\hline (11) & (52) & (53) & (54) & (55) \\
\hline (50) & (49) & (48) & (47) & (46) \\
\hline (411) & (42) & (43) & (44) & (45) \\
\hline (40) & (39) & (38) & (37) & (36) \\
\hline (11) & (32) & (33) & (34) & (35) \\
\hline (30) & (29) & (28) & (27) & (26) \\
\hline (21) & (22) & (23) & (24) & (25) \\
\hline (20) & (1) & (18) & (17) & (16) \\
\hline (11) & (12) & (13) & (e) & \\
\hline (1) & (9) & (8) & (7) & (6) \\
\hline (1) & (2) & (3) & (4) & \\
\hline
\end{tabular}

$16 \mathrm{~mm} \times 100 \mathrm{~mm}$ Tubes

\begin{tabular}{|c|c|c|c|}
\hline \multicolumn{2}{|c|}{ Peak \# } & Start Tube & End Tube \\
\hline \multicolumn{2}{|l|}{1} & A:3 & A:3 \\
\hline \multicolumn{2}{|l|}{2} & $A: 4$ & $A: 4$ \\
\hline \multicolumn{2}{|l|}{3} & A:5 & A:5 \\
\hline \multicolumn{2}{|l|}{4} & A:6 & A:7 \\
\hline \multicolumn{2}{|l|}{5} & $A: 10$ & $A: 10$ \\
\hline \multicolumn{2}{|l|}{6} & $A: 11$ & A:11 \\
\hline \multicolumn{2}{|l|}{7} & $A: 12$ & $A: 12$ \\
\hline \multicolumn{2}{|l|}{8} & $A: 13$ & $A: 13$ \\
\hline \multicolumn{2}{|l|}{9} & $A: 14$ & $A: 15$ \\
\hline \multicolumn{2}{|l|}{$\cdots$} & $\cdots$ & •. \\
\hline Duration & $\% \mathbf{B}$ & Solvent A & Solvent B \\
\hline 0.0 & 0.0 & A2 dichlorometha & B2 methanol \\
\hline 9.9 & 9.6 & A2 dichlorometha & B2 methanol \\
\hline 0.2 & 9.7 & A2 dichlorometha & B2 methanol \\
\hline & & & \\
\hline & & & \\
\hline & & & \\
\hline & & & \\
\hline
\end{tabular}


Figure S27. 5,6-Bis-(4-cyclopropyl-phenyl)-3-\{6-[5-(2,4,6-trifluoro-phenyl)-2H-pyrazol-3-yl]-pyridin-2-yl\}-

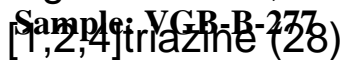

4x Column: 2

RediSep Column: Silica 4g

Flow Rate: $18 \mathrm{ml} / \mathrm{min}$

Equilibration Volume: $33.6 \mathrm{ml}$

Initial Waste: $0.0 \mathrm{ml}$

Air Purge: 0.5 min

Solvent: $\mathrm{A} 1 \mathrm{CH}_{3} \mathrm{CN}$

\section{Rf 200}

Peak Tube Volume: Max.

Non-Peak Tube Volume: Max.

Loading Type: Solid

Wavelength 1 (red): 254nm

Peak Width: $30 \mathrm{sec}$

Threshold: 0.20 AU

Wavelength 2 (purple): $280 \mathrm{~nm}$
Tuesday 29 January 2019 11:02AM

All Wavelength (orange): 200-300nm

Peak Width: 30 sec

Threshold: 0.20 AU

Run Notes:

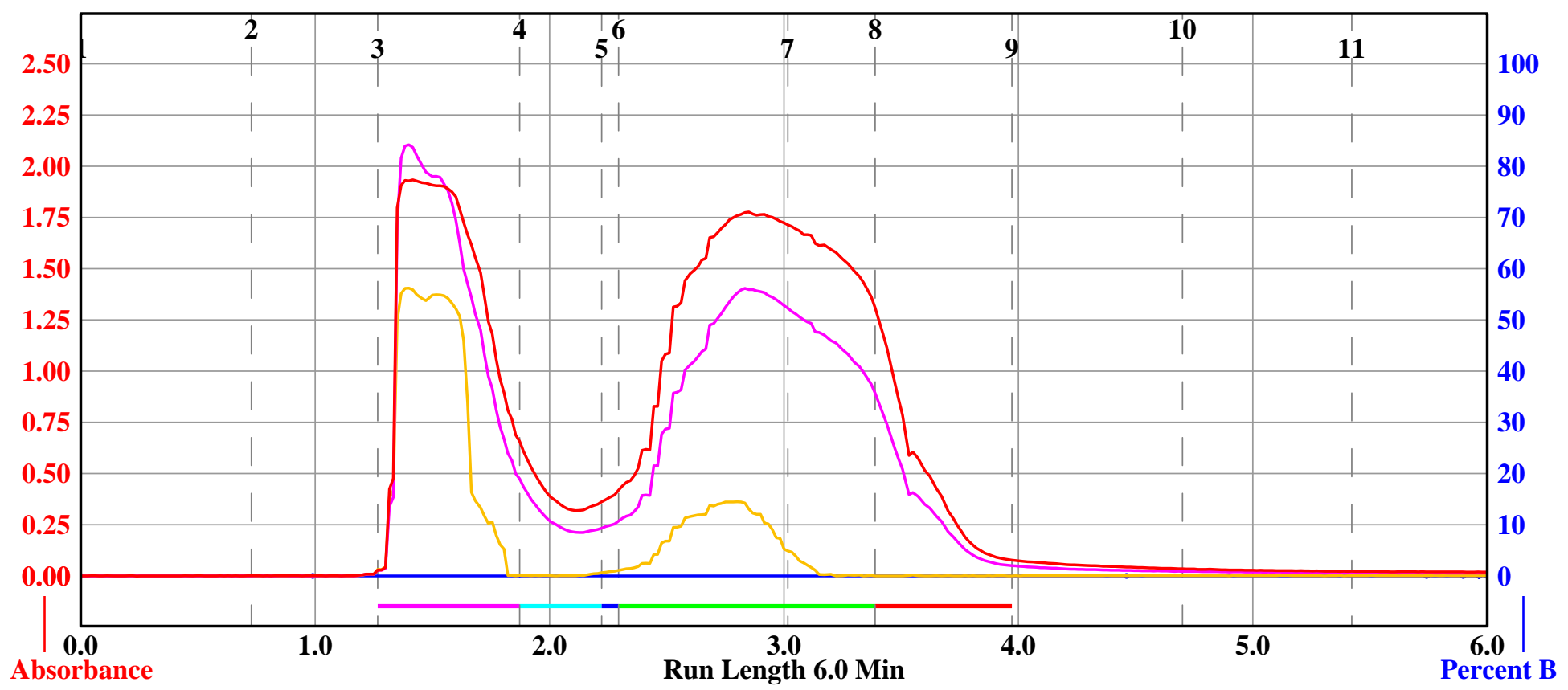

Rack A

\begin{tabular}{|c|c|c|c|}
\hline (71) & (72) & (73) & (74) \\
\hline (70) & (69) & (68) & (67) \\
\hline (61) & (62) & (63) & (64) \\
\hline (6) & (59) & (58) & (57) \\
\hline (51) & (52) & (53) & (54) \\
\hline (90) & (49) & (48) & (47) \\
\hline (41) & (42) & (43) & (44) \\
\hline (40) & (39) & (38) & (37) \\
\hline (31) & (32) & (33) & (34) \\
\hline 30 & (29) & (28) & (27) \\
\hline (21) & (22) & (23) & (24) \\
\hline (20) & (19) & (18) & (17) \\
\hline (11) & (12) & (13) & (14) \\
\hline (10) & (9) & (8) & (7) \\
\hline (1) & (2) & (3) & (4) \\
\hline
\end{tabular}

$16 \mathrm{~mm} \times 100 \mathrm{~mm}$ Tubes

\begin{tabular}{|c|c|c|c|}
\hline \multicolumn{2}{|c|}{ Peak \# } & Start Tube & End Tube \\
\hline $\begin{array}{l}1 \\
2 \\
3 \\
4 \\
5\end{array}$ & & $\begin{array}{l}A: 3 \\
A: 4 \\
A: 5 \\
A: 6 \\
A: 8\end{array}$ & $\begin{array}{l}A: 3 \\
A: 4 \\
A: 5 \\
A: 7 \\
A: 8\end{array}$ \\
\hline Duration & $\%$ B & Solvent A & Solvent B \\
\hline $\begin{array}{l}0.0 \\
1.0 \\
3.5 \\
1.3 \\
0.2 \\
0.1 \\
0.0 \\
0.0\end{array}$ & $\begin{array}{l}\mathbf{0 . 0} \\
\mathbf{0 . 0} \\
\mathbf{0 . 0} \\
\mathbf{0 . 0} \\
\mathbf{0 . 0} \\
\mathbf{0 . 0} \\
\mathbf{0 . 0} \\
\mathbf{0 . 0}\end{array}$ & $\begin{array}{l}\text { A1 hexane } \\
\text { A1 hexane } \\
\text { A1 hexane } \\
\text { A1 hexane } \\
\text { A1 hexane } \\
\text { A1 hexane } \\
\text { A1 hexane } \\
\text { A1 hexane }\end{array}$ & $\begin{array}{l}\text { B1 ethyl acetate } \\
\text { B1 ethyl acetate } \\
\text { B1 ethyl acetate } \\
\text { B1 ethyl acetate } \\
\text { B1 ethyl acetate } \\
\text { B1 ethyl acetate } \\
\text { B1 ethyl acetate } \\
\text { B1 ethyl acetate }\end{array}$ \\
\hline
\end{tabular}


Figure S28. 3-(6-\{5-[4-(4-tert-Butyl-phenylethynyl)-phenyl]-2H-pyrazol-3-y|\}-pyridin-2-yl)-5, 6-diphenylSample: VGB-C-5iliazine (29)

4x Column: 4

RediSep Column: Silica 4g

Flow Rate: $18 \mathrm{ml} / \mathrm{min}$

Equilibration Volume: $33.6 \mathrm{ml}$

Initial Waste: $0.0 \mathrm{ml}$

Air Purge: $0.5 \mathrm{~min}$

Solvent: A1 hexane

Solvent: B1 ethyl acetate
Peak Tube Volume: Max.

Non-Peak Tube Volume: Max.

Loading Type: Solid

Wavelength 1 (red): $254 \mathrm{~nm}$

Peak Width: 30 sec

Threshold: 0.20 AU

Wavelength 2 (purple): $280 \mathrm{~nm}$

Run Notes:

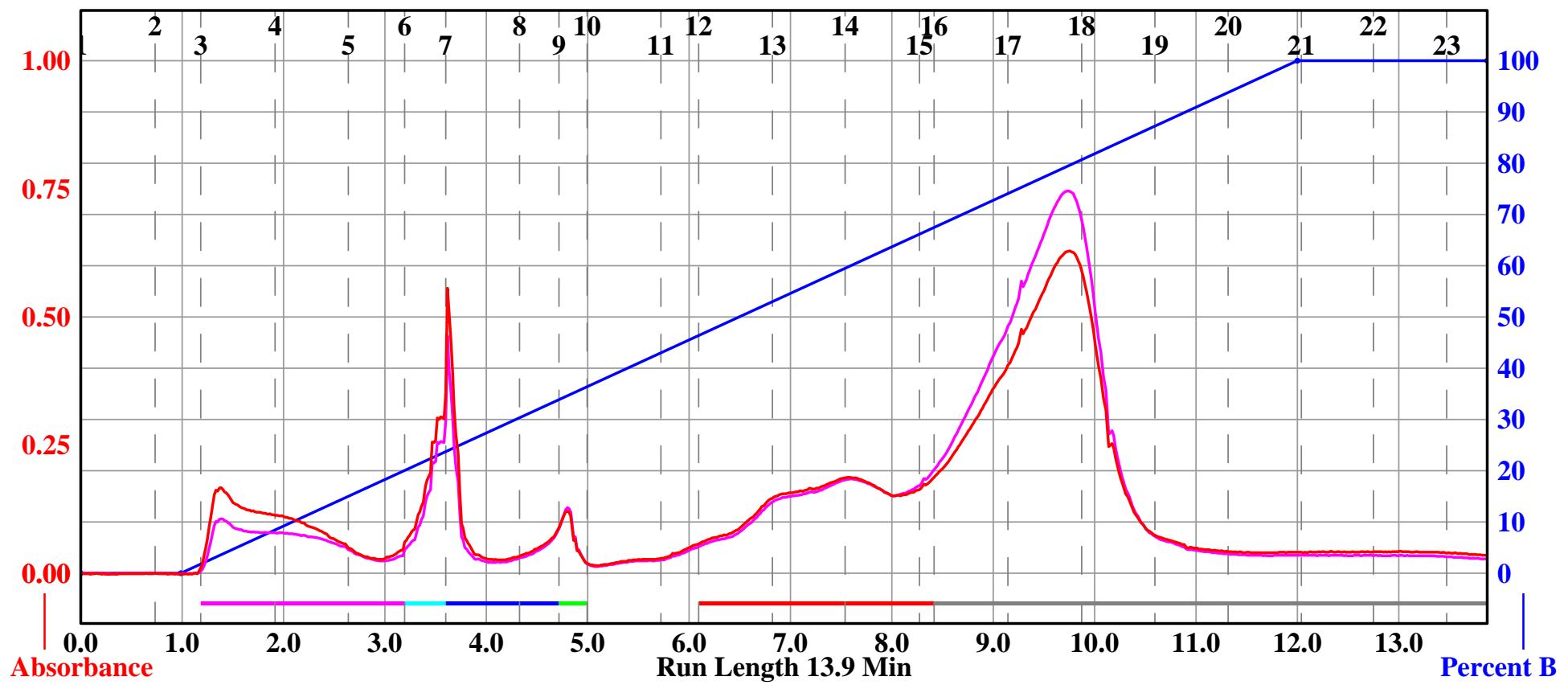

Rack A

\begin{tabular}{|c|c|c|c|}
\hline (71) & (72) & (13) & (14) \\
\hline (70) & (69) & (68) & (67) \\
\hline (61) & (62) & (63) & (64) \\
\hline (60) & (59) & (58) & (57) \\
\hline (51) & (52) & (53) & (54) \\
\hline (50) & (49) & (48) & (47) \\
\hline (41) & (42) & (43) & (44) \\
\hline (40) & (39) & (38) & (37) \\
\hline (3. & (32) & (33) & (34) \\
\hline (30) & (29) & (28) & (27) \\
\hline (21) & (22) & (23) & (24) \\
\hline (20) & (19) & (18) & (17) \\
\hline (111) & (12) & (13) & (14) \\
\hline (10) & (9) & & \\
\hline (1) & (2) & (3) & (4) \\
\hline
\end{tabular}

Peak \#

Start Tube

End Tube

\begin{tabular}{|c|c|c|c|}
\hline $\begin{array}{l}1 \\
2 \\
3 \\
4 \\
5 \\
6\end{array}$ & & $\begin{array}{c}\text { A:3 } \\
\text { A:6 } \\
\text { A:7 } \\
\text { A:9 } \\
\text { A:12 } \\
\text { A:16 }\end{array}$ & $\begin{array}{r}\text { A:5 } \\
\text { A:6 } \\
\text { A:8 } \\
\text { A:9 } \\
\text { A:15 } \\
\text { A:23 }\end{array}$ \\
\hline Duration & $\% \mathbf{B}$ & Solvent A & Solvent B \\
\hline $\begin{array}{c}0.0 \\
1.0 \\
11.0 \\
1.9\end{array}$ & $\begin{array}{c}0.0 \\
0.0 \\
100.0 \\
100.0\end{array}$ & $\begin{array}{l}\text { A1 hexane } \\
\text { A1 hexane } \\
\text { A1 hexane } \\
\text { A1 hexane }\end{array}$ & $\begin{array}{l}\text { B1 ethyl acetate } \\
\text { B1 ethyl acetate } \\
\text { B1 ethyl acetate } \\
\text { B1 ethyl acetate }\end{array}$ \\
\hline
\end{tabular}




\section{MS Data Review Active Chromatogram and Spectrum Plots - 4/2/2019 4:19 PM}

File: c:Ittulcarricklgirildatal121318l19 80h2o_20acn 100acn 33min curve 0_2ml 121218 12-13-2018 10-14-30 am.xms Sample: 19

Scan Range: 1 - 3256 Time Range: 0.03 - $29.00 \mathrm{~min} . \quad$ Date: 12/13/2018 10:14 AM

Operator: GM

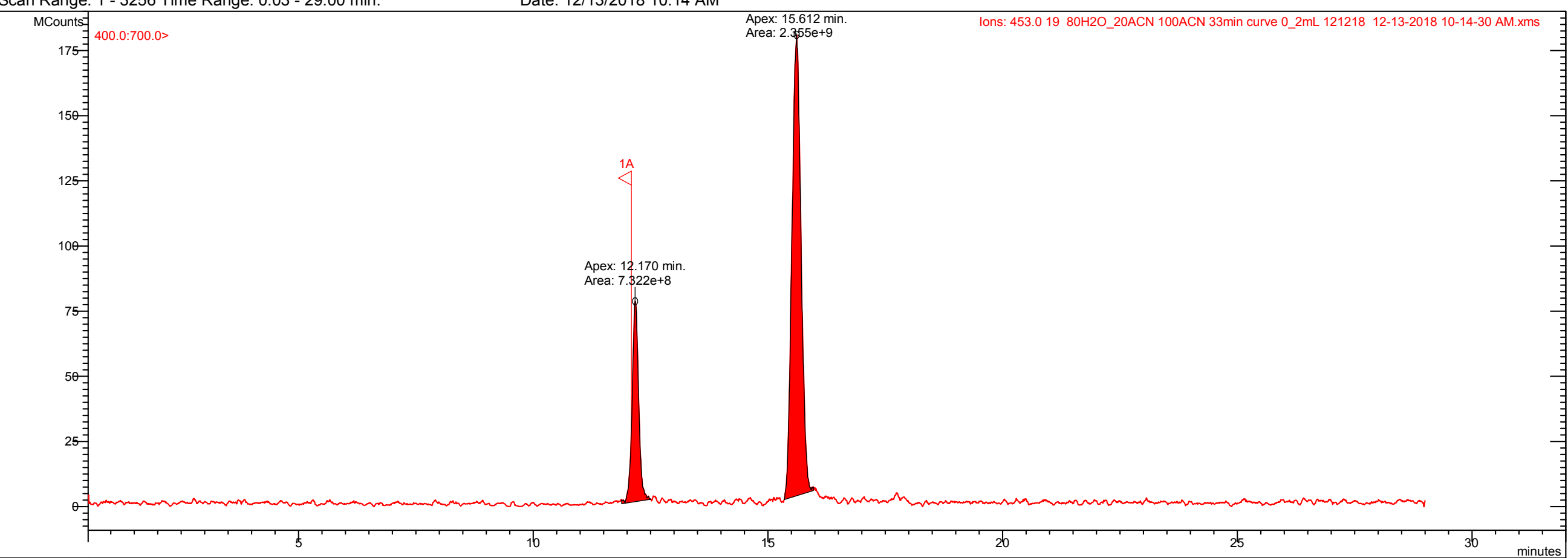

BP: 453.2 (2.285e+7=100\%), 19 80h20 20acn 100acn 33min curve 0 2 $2 \mathrm{ml} 121218 \quad 12$ -

$12.091 \mathrm{~min}$, Scan: $1334,400.0: 700.0>$, Ion: NA, RIC: $8.860 \mathrm{e}+7, \mathrm{BC}$

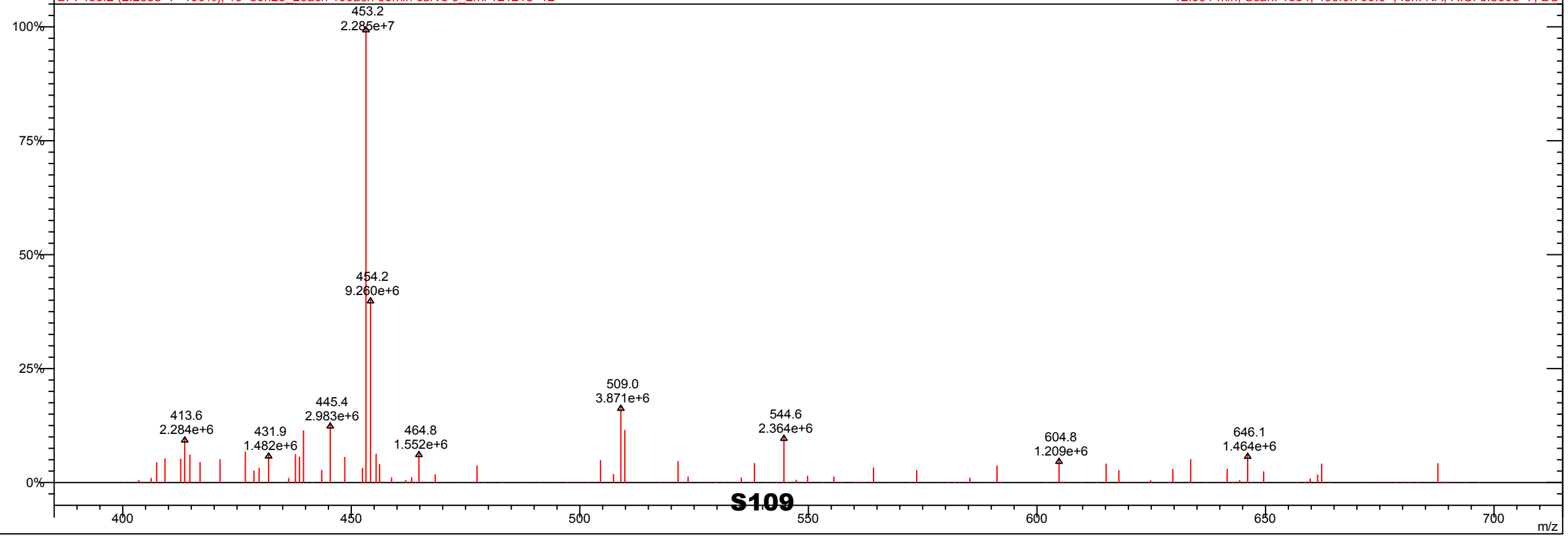




\section{Print Date: 26 Apr 2019 15:58:18 Figure S30. 5,6-Diphenyl-3-[6-(5-p-tolyl-2H-pyrazol-3-yl)-pyridin-2-yl]-[1,2,4]triazine (2)}

\section{MS Data Review Active Chromatogram and Spectrum Plots - 4/26/2019 3:57 PM}

File: c:Ittulcarricklgirildatal042519lc-31 80h2o_20acn 100acn 45min 2min_ramp 0_2ml 121218 4-25-2019 9-54-46 am.xms Sample: C-31

Operator: GM

Scan Range: 1 - 3794 Time Range: 0.04 - 41.00 min.

Date: 4/25/2019 9:54 AM

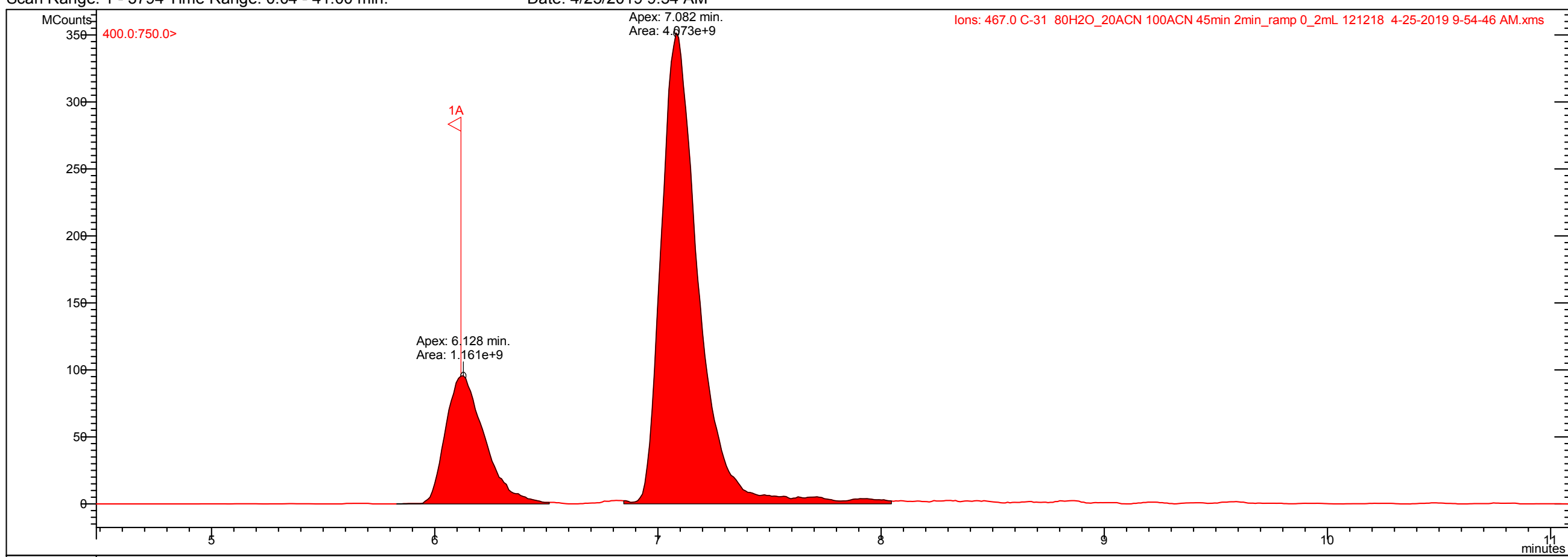

SPectrum $1 \mathrm{~A}$
BP: $467.2(1.083 \mathrm{e}+8=100 \%)$, c-31 $80 \mathrm{~h} 20 \quad 20 \mathrm{acn}$ 100acn $45 \mathrm{~min} 2 \mathrm{~min}$ ramp $0.2 \mathrm{ml} 12121$

6.117 min, Scan: $527,400.0: 750.0>$, Ion: NA, RIC: $1.537 \mathrm{e}+8, \mathrm{BC}$

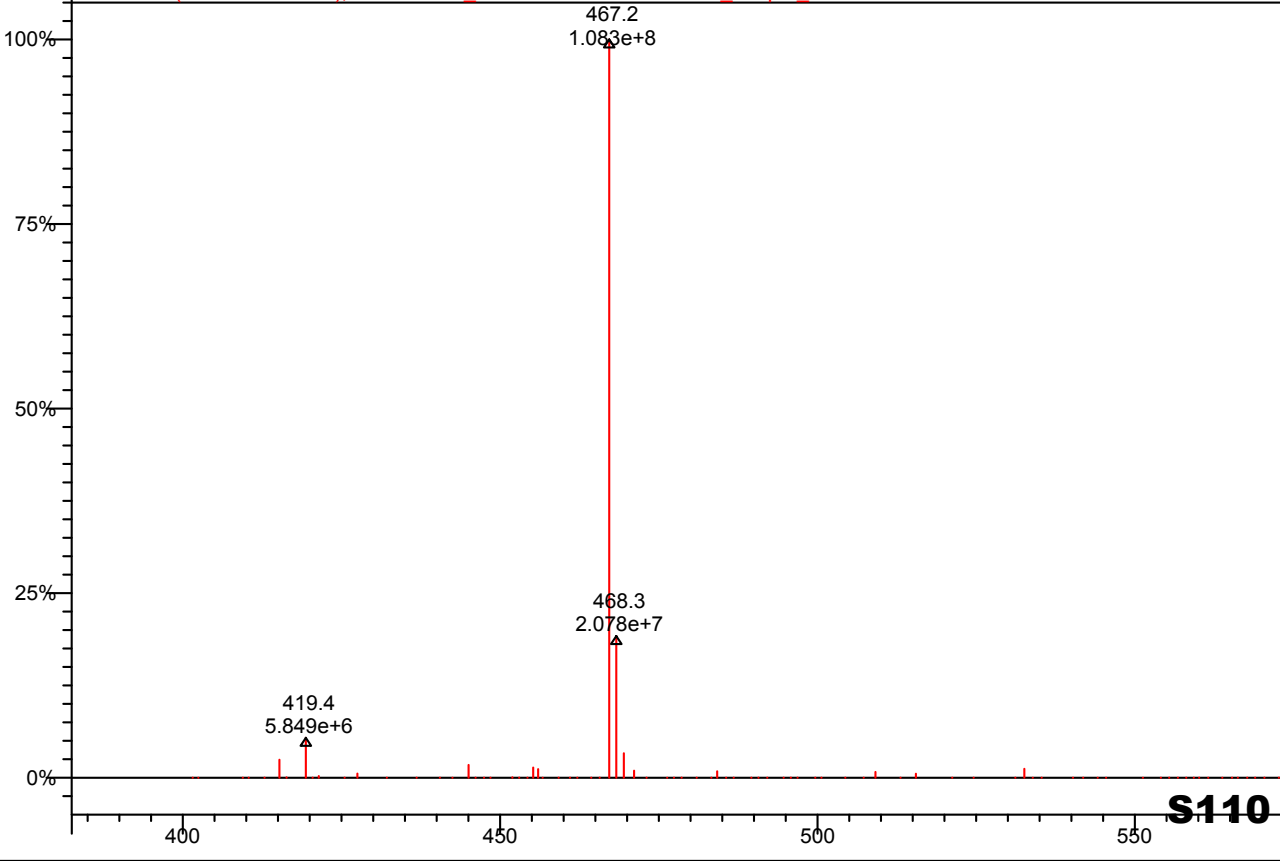

S110 


\section{MS Data Review Active Chromatogram and Spectrum Plots - 5/1/2019 10:14 AM}

File: c:Ittulcarricklgirildatal041819lb-225 80h2o_20acn 100acn 45min 2min_ramp 0_2ml 121218 4-18-2019 10-16-12 am.xms Sample: B-225

Scan Range: 1 - 3656 Time Range: 0.03 - 37.42 min. Date: 4/18/2019 10:16 AM Operator: GM

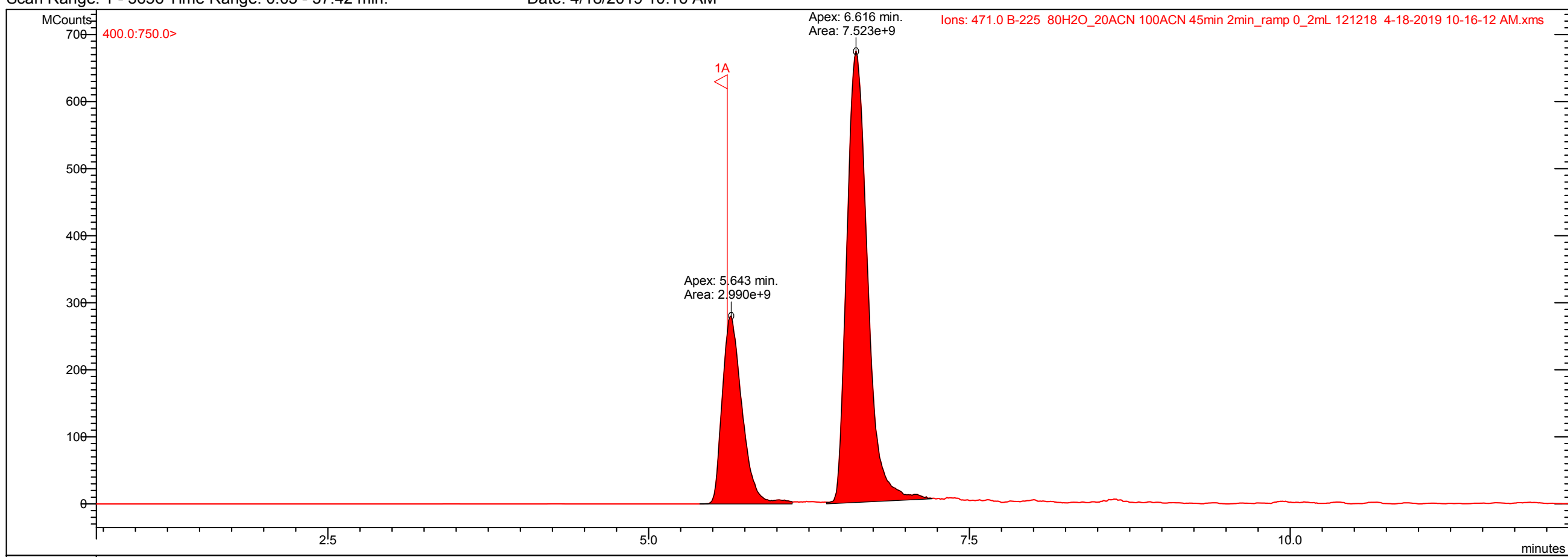

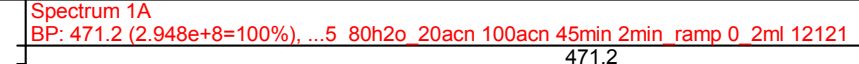

5.613 min, Scan: $505,400.0: 750.0>$, Ion: NA, RIC: $4.235 \mathrm{e}+8$, BC

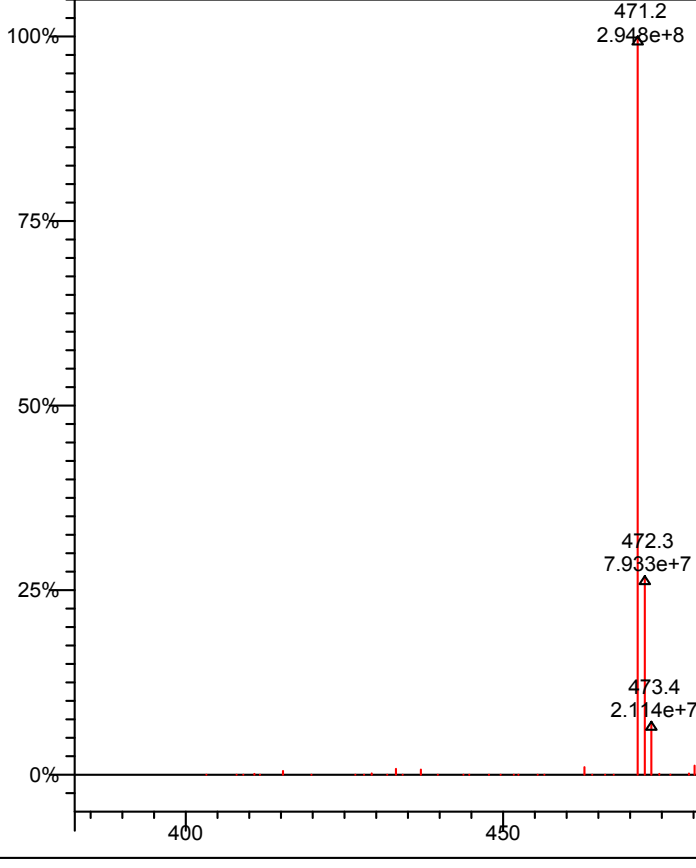

471.2
$2.948 \mathrm{e}+8$

472.3
$7.933 \mathrm{e}+7$

3.4
$1 \mathrm{e}+7$ 
Print Date: 02 Apr 2019 15:42:17 Figure S32. 5,6-Diphenyl-3-\{6-[5-(4-trifluoromethyl-phenyl)-2H-pyrazol-3-yl]-pyridin-2-yl\}-[1,2,4]triazine (11)

\section{MS Data Review Active Chromatogram and Spectrum Plots - 4/2/2019 3:41 PM}

File: c:lttulcarricklgirildatal020119lb251 80h2o_20acn 100acn 37min curve 0_2ml 121218 2-1-2019 1-20-07 pm.xms Sample: B251

Operator: GM

Scan Range: 1 - 3514 Time Range: 0.03 - $32.01 \mathrm{~min}$

Date: 2/1/2019 1:20 PM

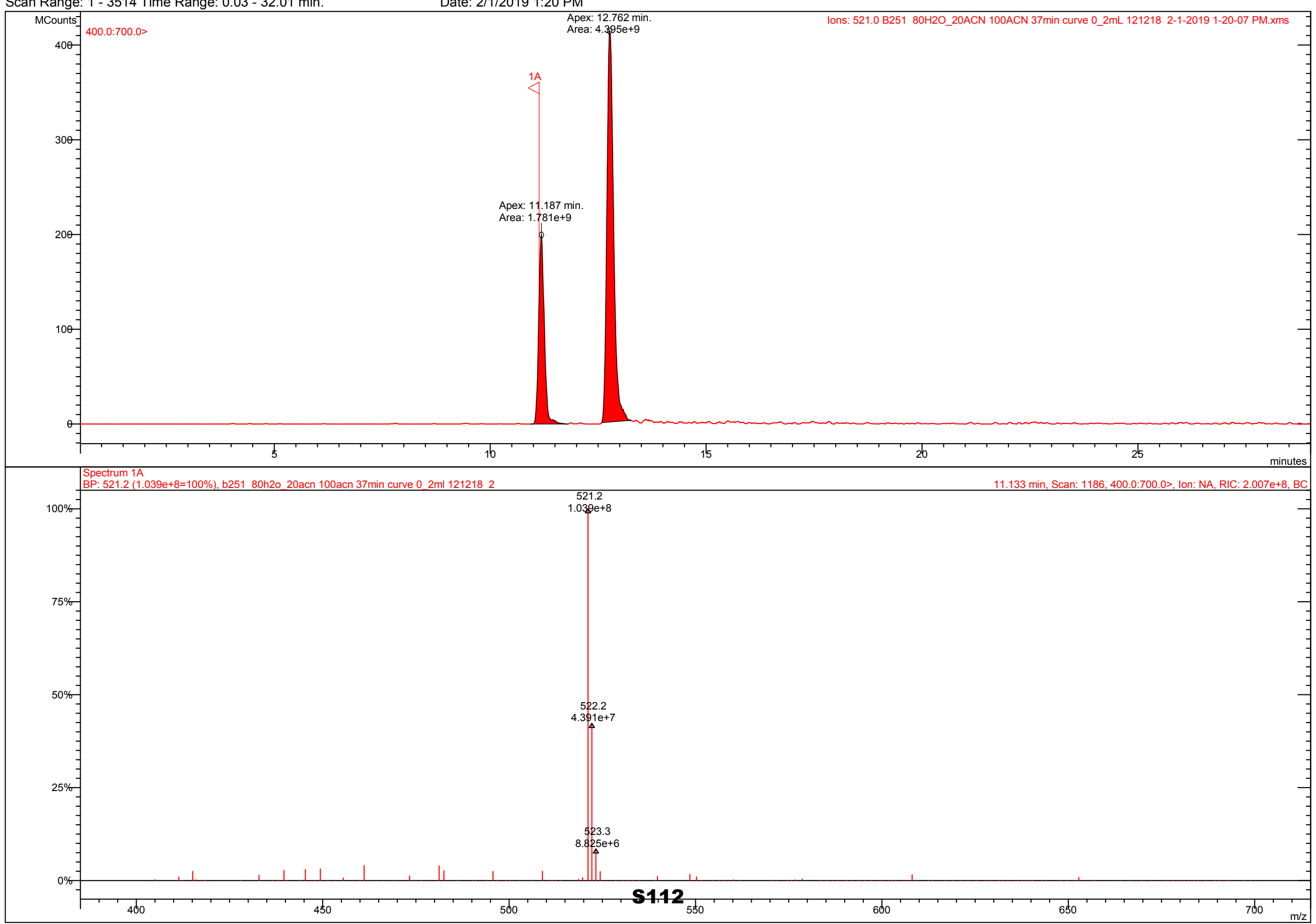




\section{Print Date: 12 Apr 2019 11:21:17 Figure S33. 3-\{6-[5-(4-Bromo-phenyl)-2H-pyrazol-3-yl]-pyridin-2-yl\}-5,6-diphenyl-[1,2,4]triazine (12)}

\section{MS Data Review Active Chromatogram and Spectrum Plots - 4/12/2019 11:20 AM}

File: c:Ittulcarricklgirildatal0405191040819lb-243 80h2o_20acn 100acn 45min 2min_ramp 0_2ml 121218 4-9-2019 9-58-56 am.xms Sample: B-243 Operator: GM

Scan Range: 1 - 4080 Time Range: 0.03 - 41.00 min._Date: 4/9/2019 9:58 AM

Sample Notes: Pure sample
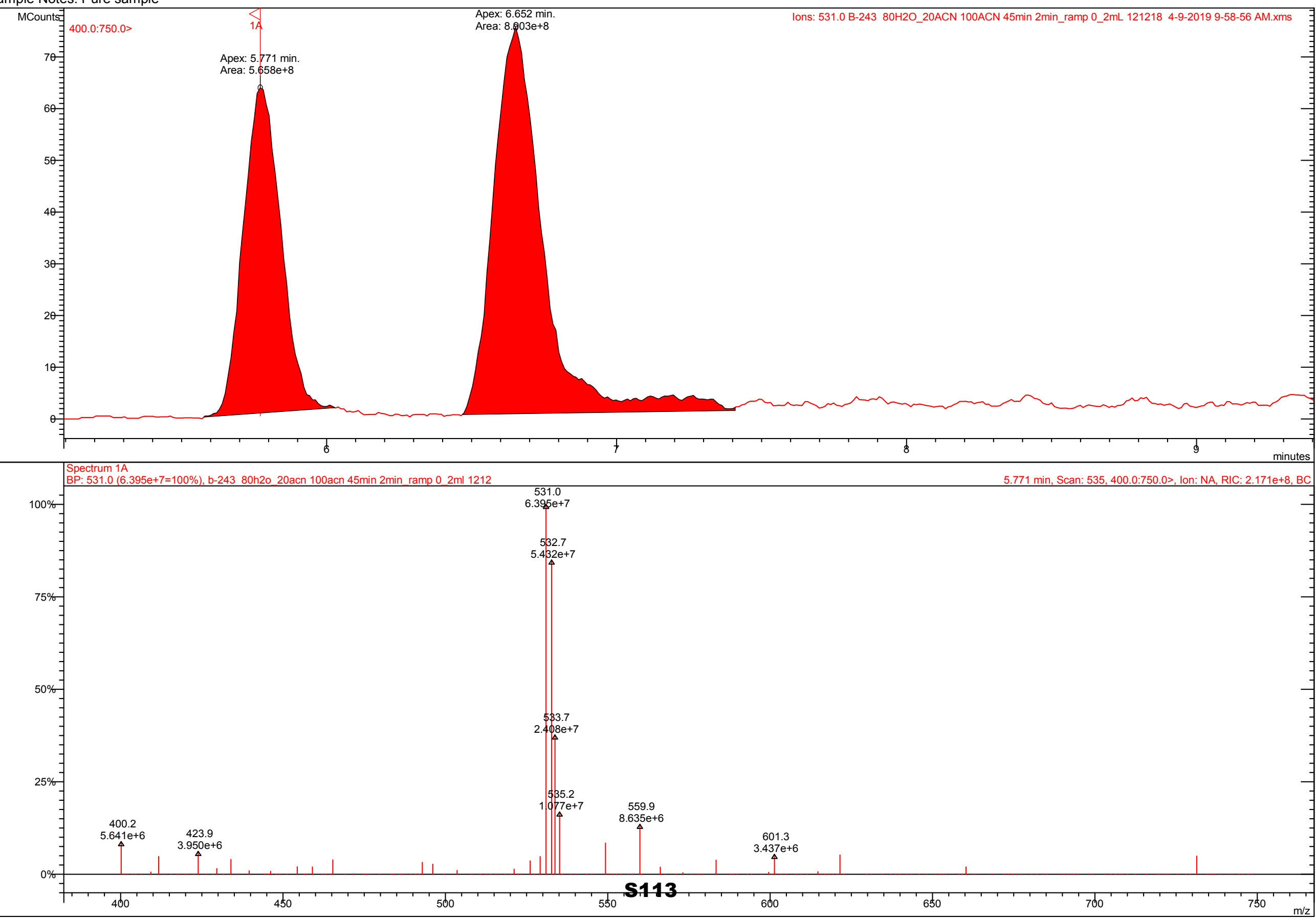


\section{Print Date: 12 Apr 2019 11:57:12 Figure S34. (4-\{5-[6-(5,6-Diphenyl-[1,2,4]triazin-3-yl)-pyridin-2-yl]-1H-pyrazol-3-yl\}-phenyl)-dimethylamine (13)}

\section{MS Data Review Active Chromatogram and Spectrum Plots - 4/12/2019 11:56 AM}

File: c:Ittulcarricklgirildatal040519l040819lc-17 80h2o_20acn 100acn 45min 2min_ramp 0_2ml 121218 4-9-2019 8-24-53 am.xms

Sample: C-17

Operator: GM

Scan Range: 1 - 4040 Time Range: 0.03 - 41.00 min _ Date: 4/9/2019 8:24 AM

Sample Notes: Pure sample

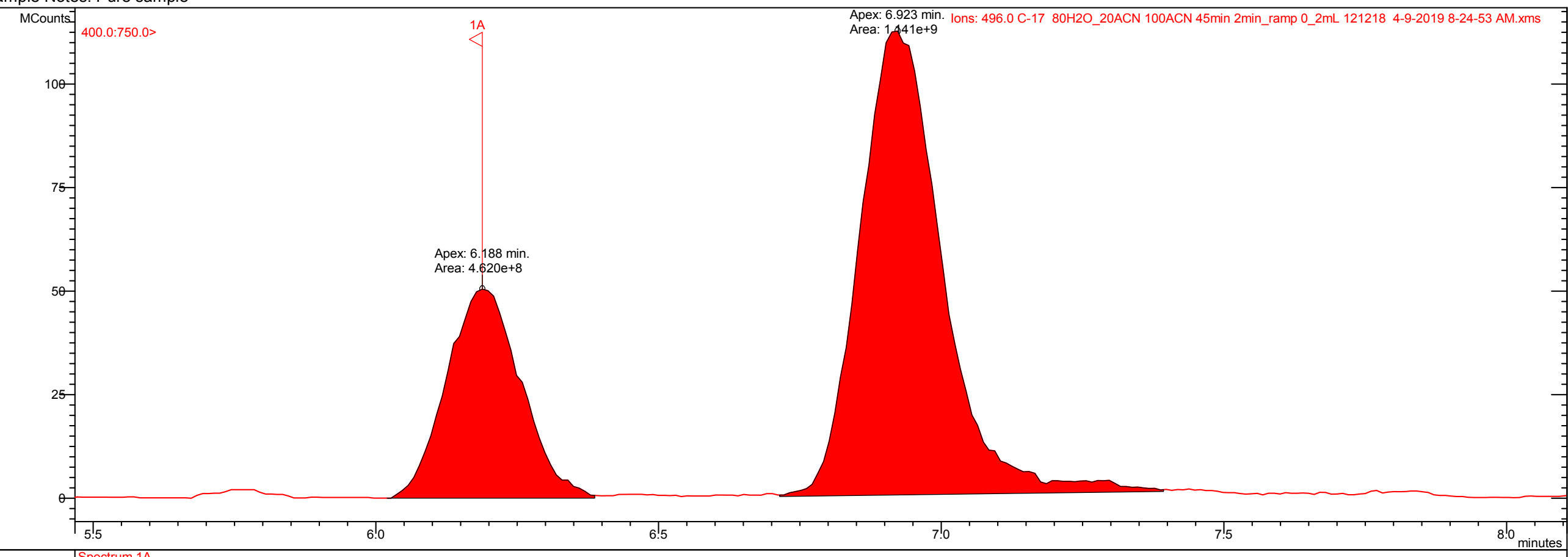

BP: 496.2 (7.109e+7=100\%), c-17 80h20 20acn 100acn 45min 2min ramp 0 $2 \mathrm{ml} 12121$

6.188 min, Scan: $567,400.0: 750.0>$, Ion: NA, RIC: $1.687 \mathrm{e}+8, \mathrm{BC}$

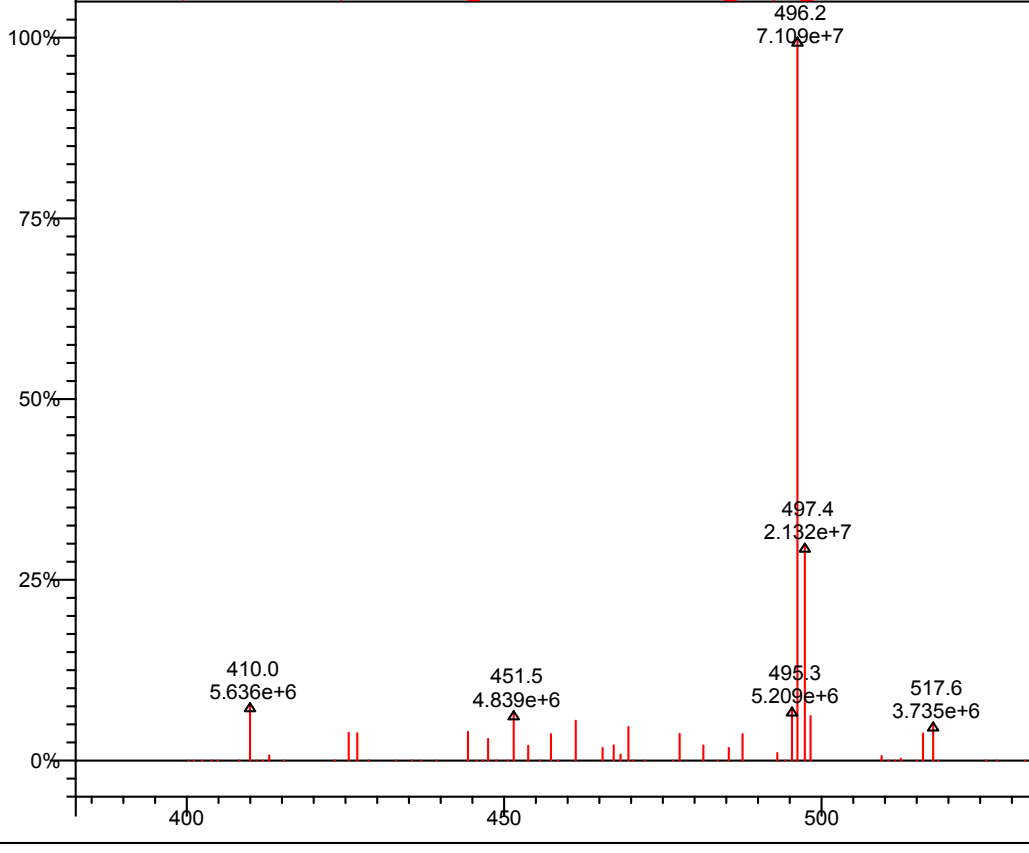

$\mathbf{S 1 1 4}$


Print Date: 03 Apr 2019 11:20:13 Figure S35 3-\{6-[5-(4-Methoxy-phenyl)-2H-pyrazol-3-yl]-pyridin-2-yl\}-5,6-diphenyl-[1,2,4]triazine (14)

\section{MS Data Review Active Chromatogram and Spectrum Plots - 4/3/2019 11:19 AM}

File: c:Ittulcarricklgirildatal020119lb203 80h2o_20acn 100acn 37min curve 0_2ml 121218 2-1-2019 11-01-16 am.xms Sample: B203 Operator: GM

Scan Range: 1 - 3542 Time Range: 0.02 - 32.00 min.

Date: 2/1/2019 11:01 AM

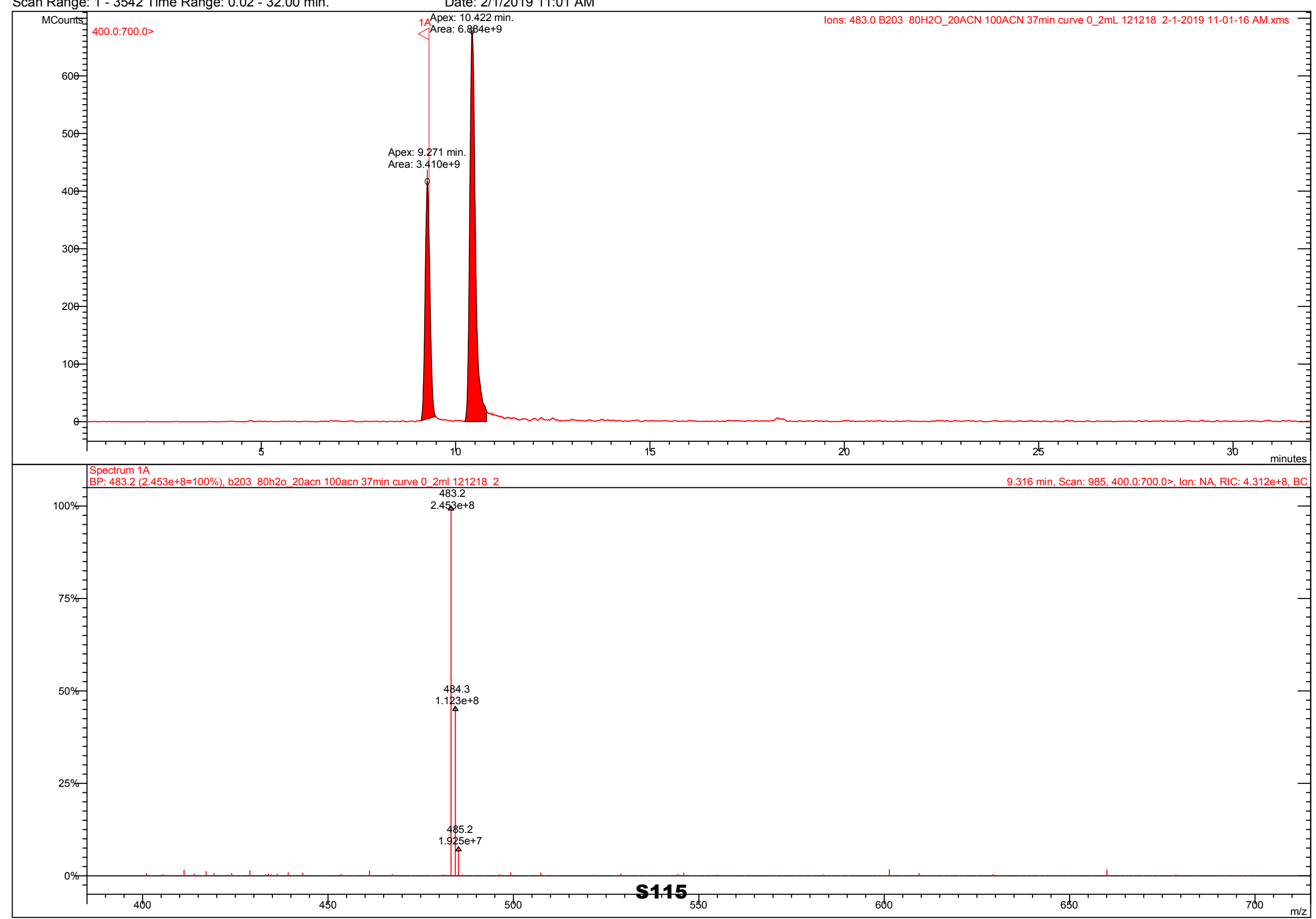


Print Date: 02 Apr 2019 15:32:40 Figure S36. 3-\{5-[6-(5,6-Diphenyl-[1,2,4]triazin-3-yl)-pyridin-2-yl]-1H-pyrazol-3-yl\}-benzoic acid methyl ester (15)

\section{MS Data Review Active Chromatogram and Spectrum Plots - 4/2/2019 3:31 PM}

File: c:Ittulcarricklgirildatal011519l215 80h2o_20acn 100acn 33min curve 0_2ml 121218 1-15-2019 4-17-20 pm.xms Sample: 215

Date: 1/15/2019 4:17 PM

Scan Range: 1 - 2804 Time Range: 0.04 - 29.00 min.

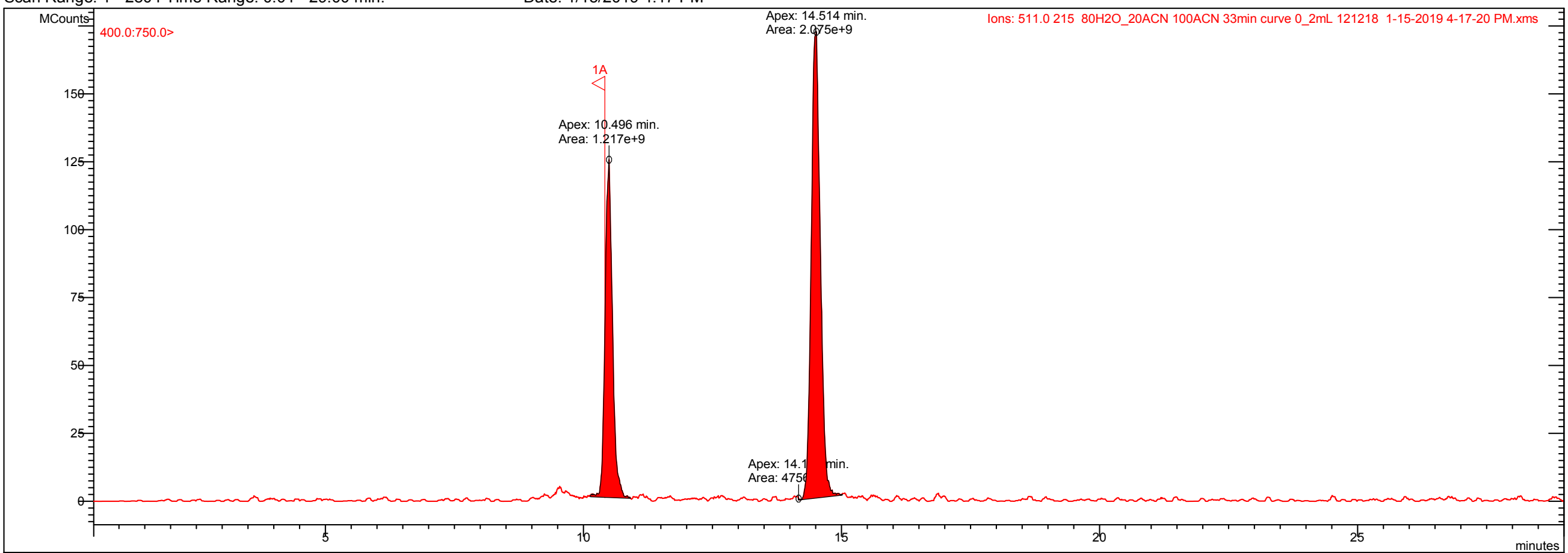

\begin{tabular}{l} 
Spectrum 1A \\
BP: $511.2(6.865 \mathrm{e}+7=100 \%), 215$ \\
\hline
\end{tabular} 10.414 min, Scan: $979,400.0: 750.0>$, Ion: NA, RIC: $2.298 \mathrm{e}+8, \mathrm{BC}$

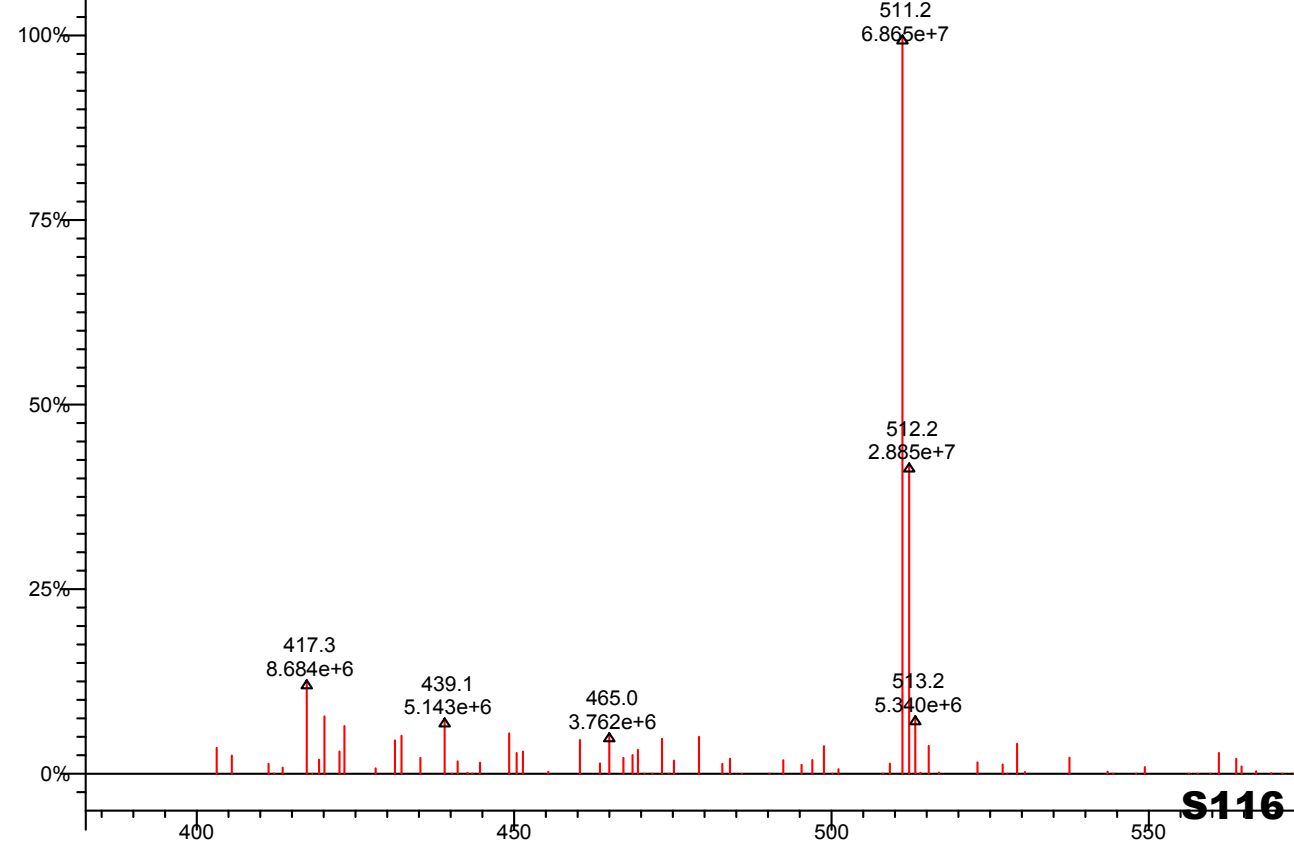


Print Date: 02 Apr 2019 15:39:38 Figure S37. 3-\{6-[5-(2-lodo-phenyl)-2H-pyrazol-3-yl]-pyridin-2-yl\}-5,6-diphenyl-[1,2,4]triazine (16)

\section{MS Data Review Active Chromatogram and Spectrum Plots - 4/2/2019 3:38 PM}

File: c:Ittulcarricklgirildatal020119lb249 80h2o_20acn 100acn 37min curve 0_2ml 121218 2-1-2019 12-41-05 pm.xms Sample: B249

Scan Range: 1 - 3486 Time Range: 0.03 - $32.01 \mathrm{~min} . \quad$ Date: 2/1/2019 12:41 PM Operator: GM
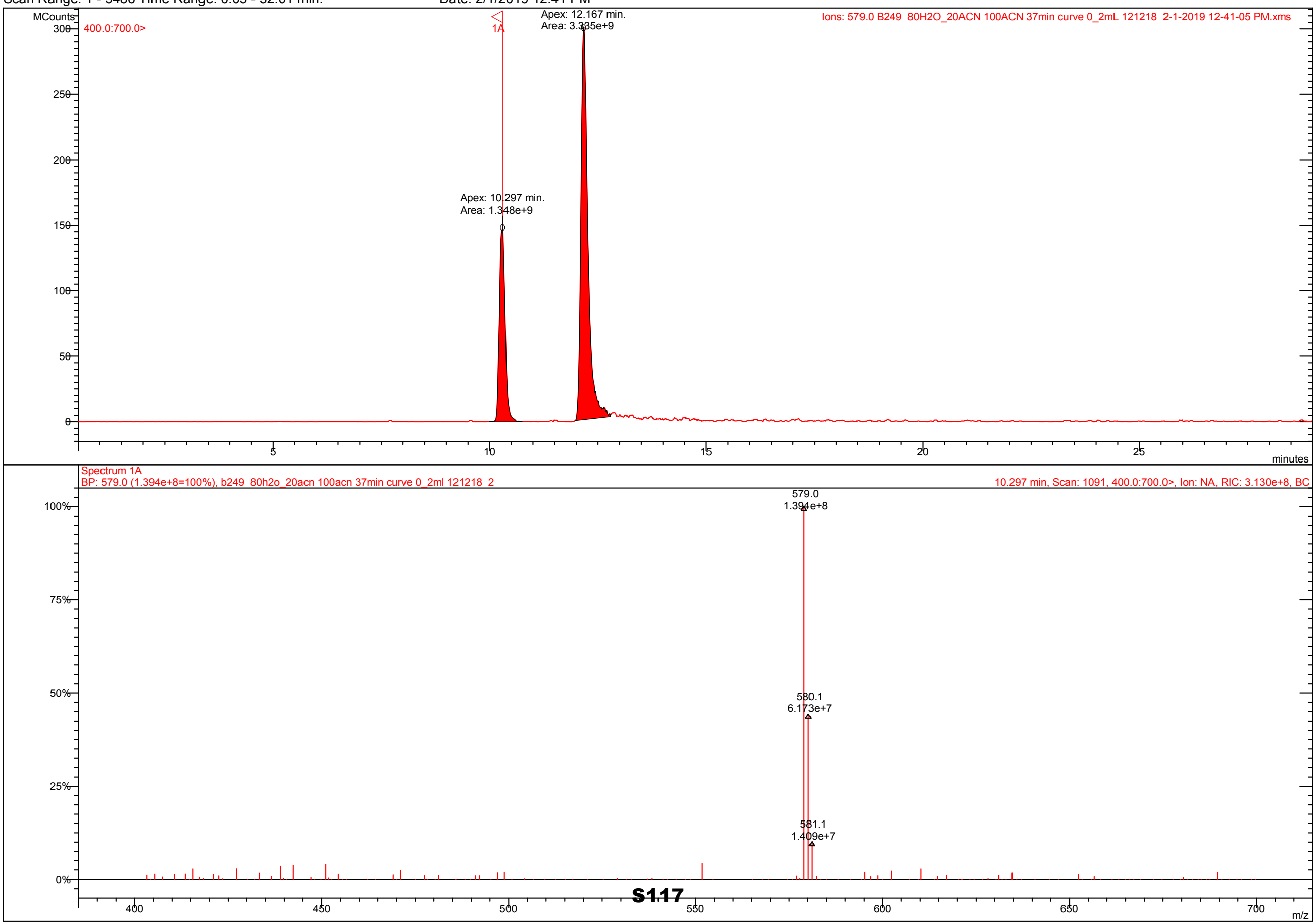
Print Date: 02 Apr 2019 19:59:17 Figure S38. 5,6-Diphenyl-3-\{6-[5-(2,4,6-trifluoro-phenyl)-2H-pyrazol-3-yl]-pyridin-2-yl\}-[1,2,4]triazine (17)

\section{MS Data Review Active Chromatogram and Spectrum Plots - 4/2/2019 7:58 PM}

File: c:Ittulcarricklgirildatal121218161 80h2o_20acn 100acn 37min curve 0_2ml 121218 12-12-2018 8-57-52 am.xms Sample: 61

Operator: GM

Scan Range: 1 - 3550 Time Range: 0.02 - 32.00 min

Date: 12/12/2018 8:57 AM

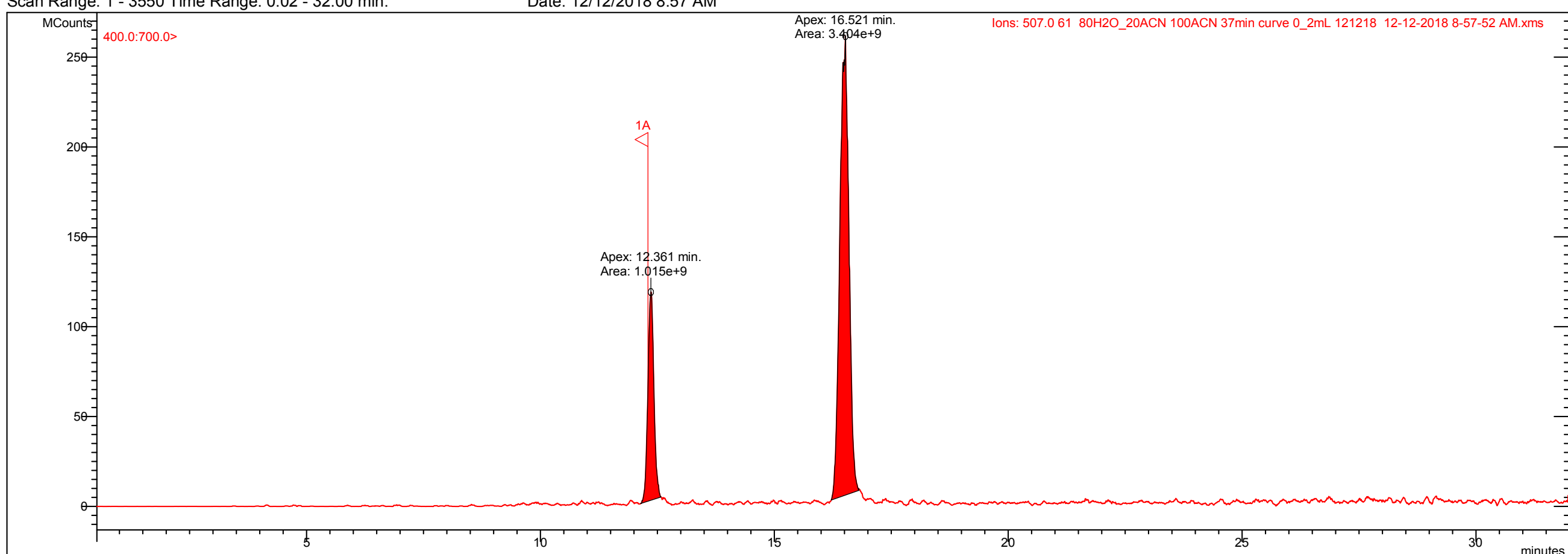

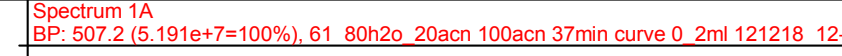

20

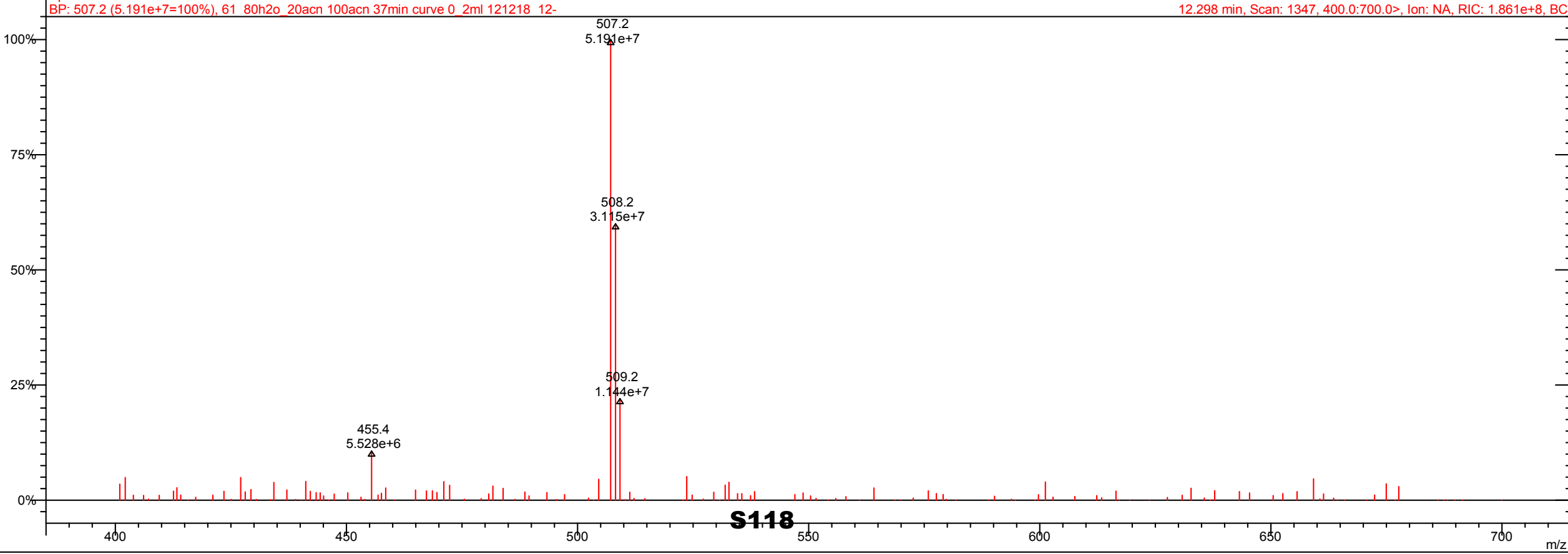




\section{Print Date: 12 Apr 2019 11:14:26 Figure S39. 3-\{6-[5-(3,5-Di-tert-butyl-phenyl)-2H-pyrazol-3-yl]-pyridin-2-yl\}-5,6-diphenyl-[1,2,4]triazine (18)}

\section{MS Data Review Active Chromatogram and Spectrum Plots - 4/12/2019 11:13 AM}

File: c:Ittulcarricklgirildatal0405191040819lb-211 80h2o_20acn 100acn 45min 2min_ramp 0_2ml 121218 4-9-2019 9-11-53 am.xms Sample: B-211 Operator: GM

Scan Range: 1 - 4090 Time Range: 0.03 - 40.99 min. Date: 4/9/2019 9:11 AM

Sample Notes: Pure sample
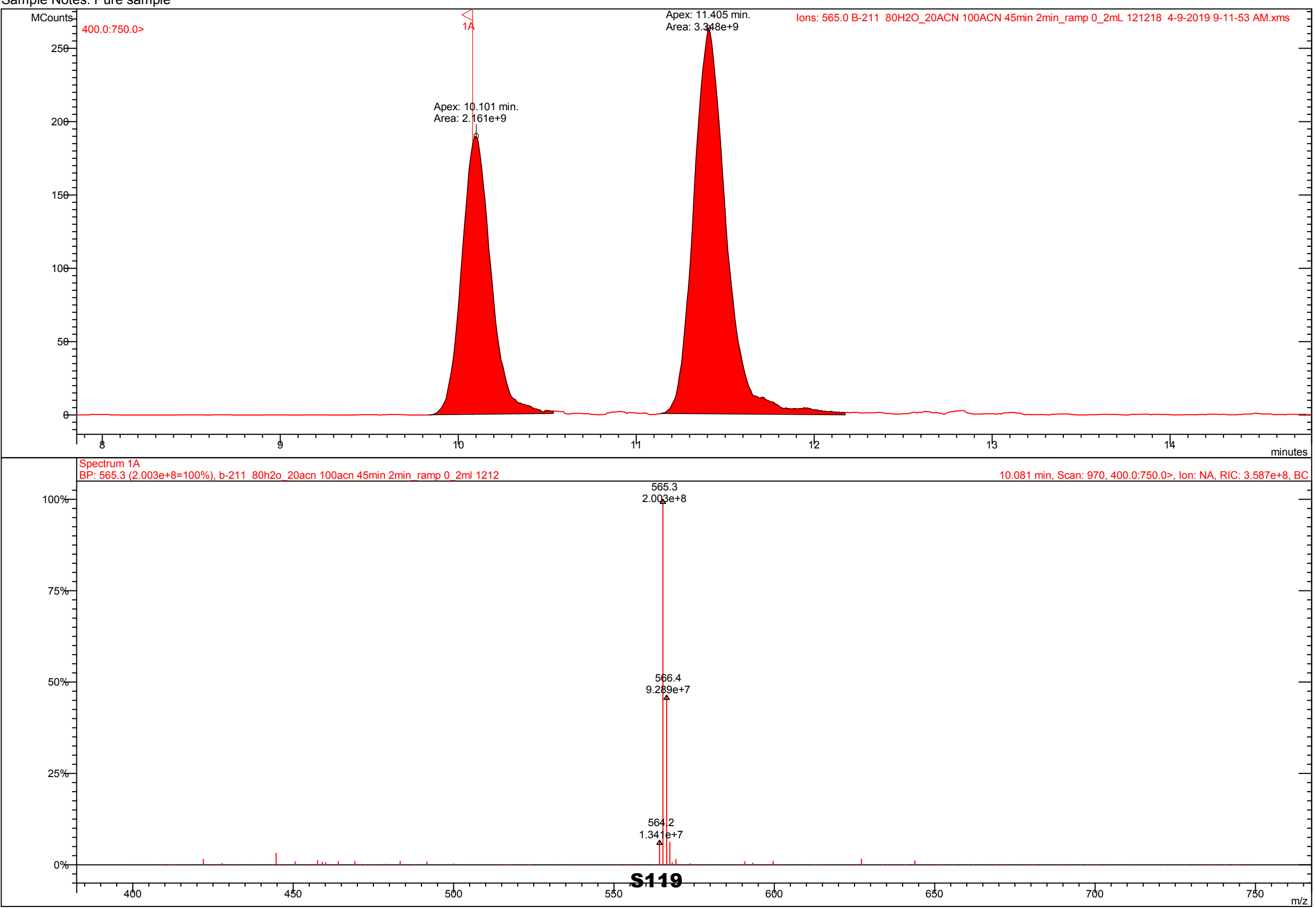
Print Date: 03 Apr 2019 11:23:14 Figure S40. 5,6-Diphenyl-3-[6-(5-pyridin-4-yl-2H-pyrazol-3-yl)-pyridin-2-yl]-[1,2,4]triazine (19)

\section{MS Data Review Active Chromatogram and Spectrum Plots - 4/3/2019 11:22 AM}

File: c:Ittulcarricklgirildatal0115191209 80h2o_20acn 100acn 33min curve 0_2ml 121218 1-15-2019 2-32-12 pm.xms Sample: 209 Operator: GM

Scan Range: 1 - 2864 Time Range: 0.03 - $29.01 \mathrm{~min} . \quad$ Date: 1/15/2019 2:32 PM

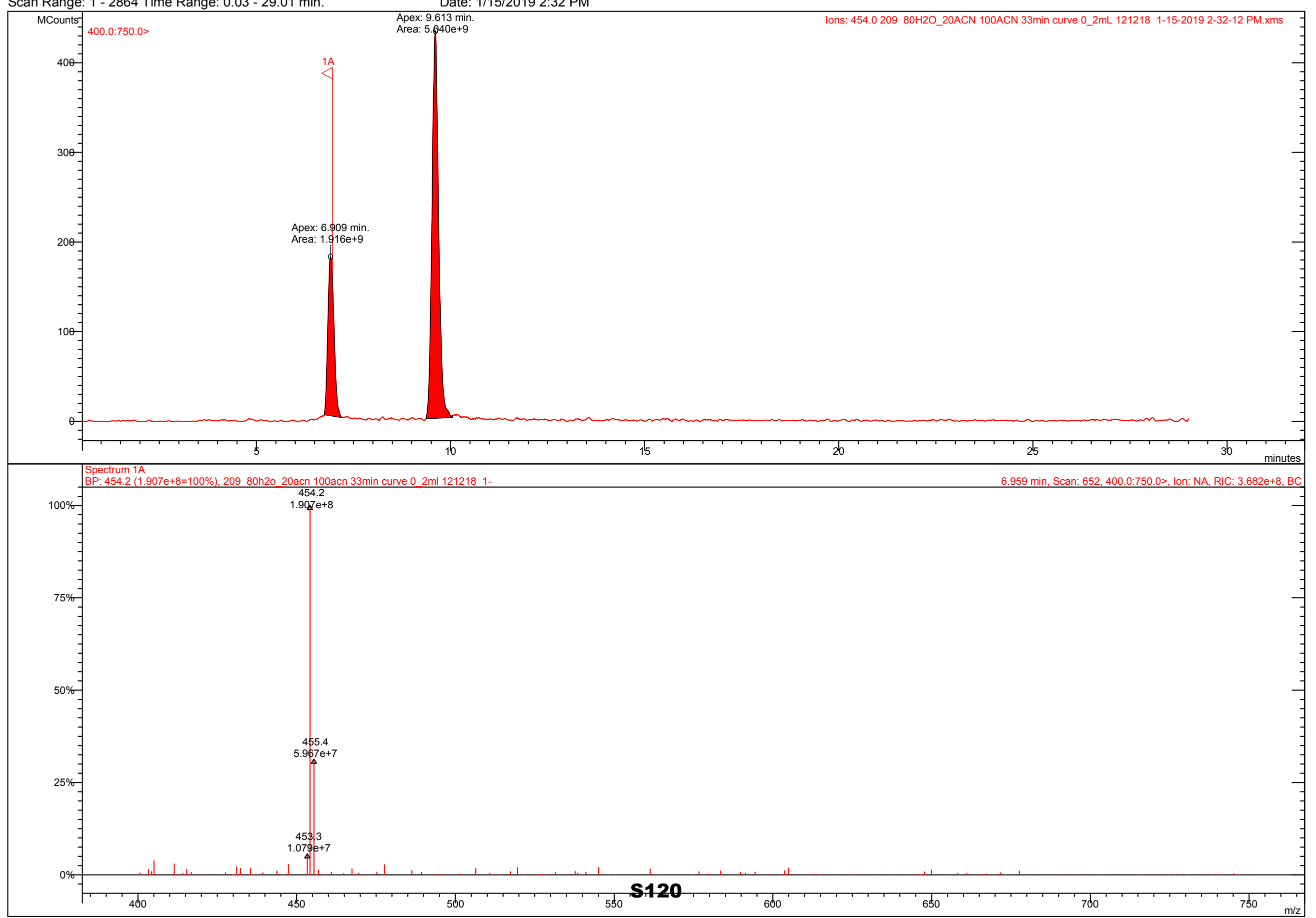


Print Date: 02 Apr 2019 15:50:41 Figure S41. 5,6-Bis-(4-cyclopropyl-phenyl)-3-[6-(5-phenyl-2H-pyrazol-3-yl)-pyridin-2-yl]-[1,2,4]triazine (20)

\section{MS Data Review Active Chromatogram and Spectrum Plots - 4/2/2019 3:50 PM}

File: c:Ittulcarricklgirildatal020119lb265 80h2o_20acn 100acn 37min curve 0_2ml 121218 2-1-2019 3-56-13 pm.xms Sample: B265

Operator: GM

Scan Range: 1 - 3492 Time Range: 0.03 - 32.00 min

Date: 2/1/2019 3:56 PM

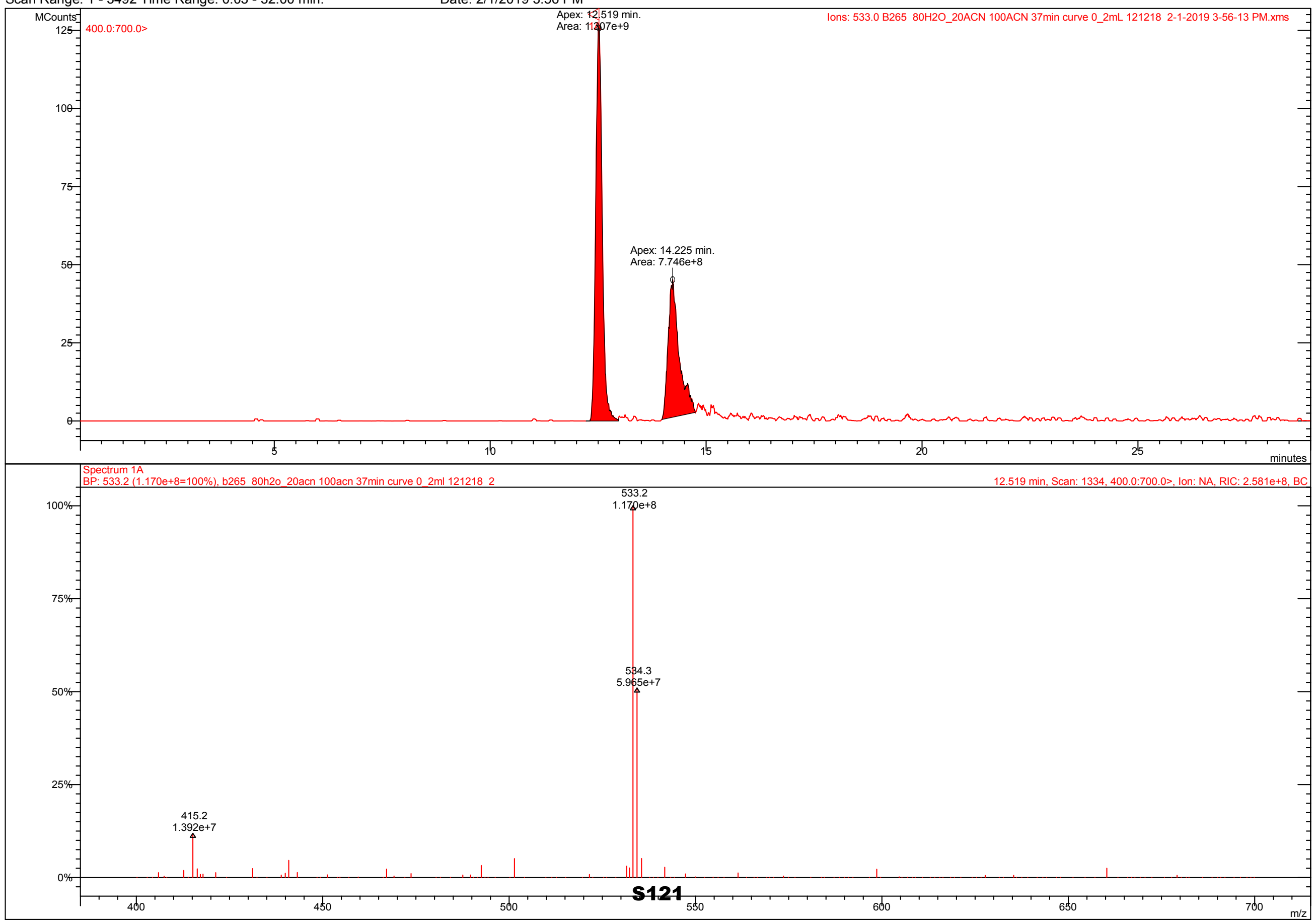


Print Date: 18 Apr 2019 10:26:32 Figure S42. 5,6-Bis-(4-cyclopropyl-phenyl)-3-\{6-[5-(2-iodo-phenyl)-2H-pyrazol-3-yl]-pyridin-2-yl\}-[1,2,4] triazine (21)

\section{MS Data Review Active Chromatogram and Spectrum Plots - 4/18/2019 10:26 AM}

File: c:Ittulcarricklgirildatal0411519lb-269 80h2o_20acn 100acn 45min 2min_ramp 0_2ml 121218 4-17-2019 9-57-28 am.xms

Sample: B-269

Date: 4/17/2019 9:57 AM

\begin{tabular}{rr} 
Scan Range: 1 - 3992 Time Range: $0.02-41.00 \mathrm{min.}$ & Date: 4/17/2019 9:57 AM \\
\hline MCountsł & Apex: $9.121 \mathrm{~min}$.
\end{tabular}
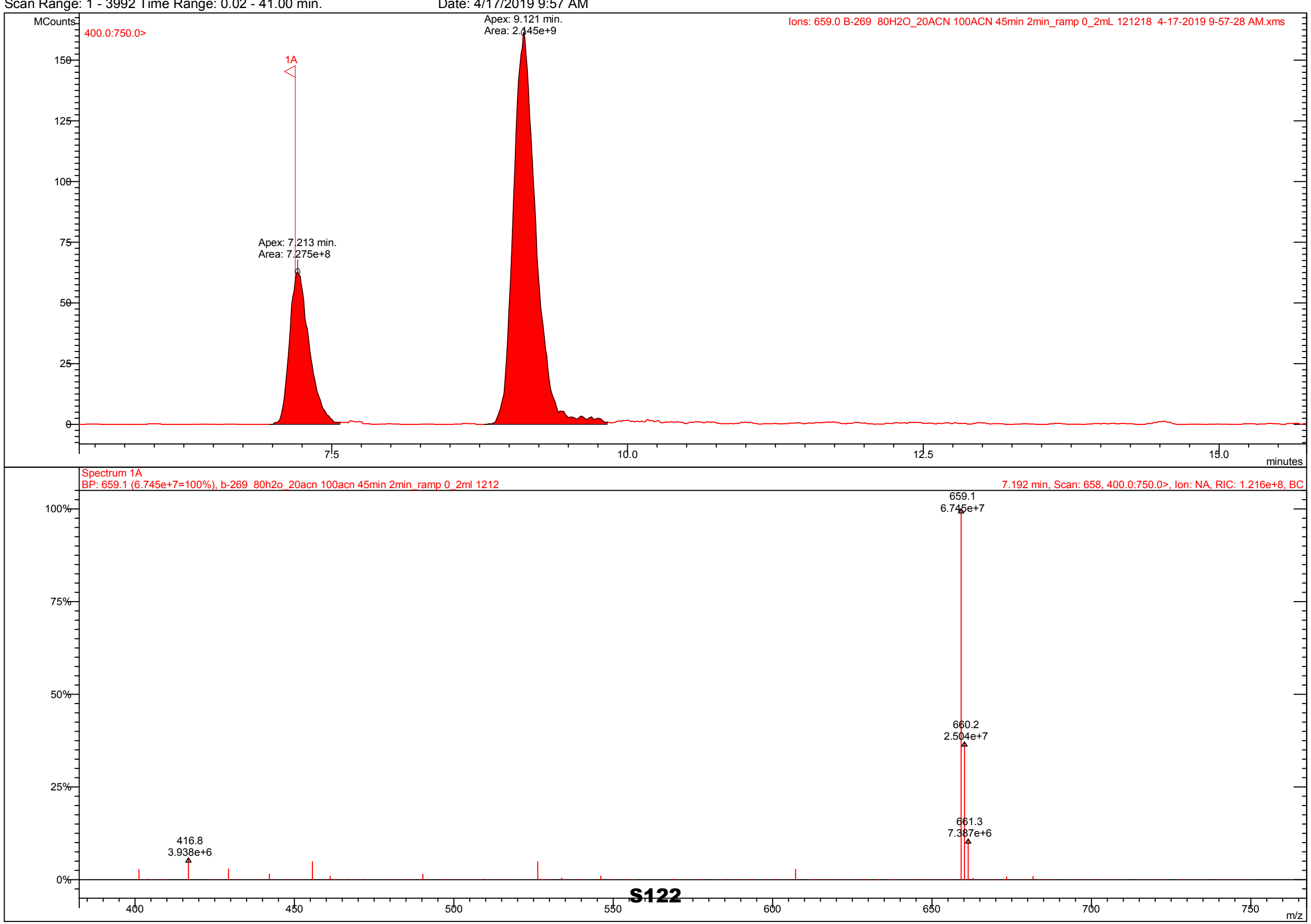
Print Date: 05 Apr 2019 10:52:18 Figure S43. 3-\{6-[5-(4-Bromo-phenyl)-2H-pyrazol-3-yl]-pyridin-2-yl\}-5,6-bis-(4-butyl-phenyl)-[1,2,4]triazine (22)

\section{MS Data Review Active Chromatogram and Spectrum Plots - 4/5/2019 10:51 AM}

File: c:Ittulcarricklgirildatal011819l221 80h2o_20acn 100acn 55min 2min_ramp 0_2ml 121218 1-18-2019 10-30-53 am.xms Sample: 221

Scan Range: 1 - 5326 Time Range: 0.04 - $55.01 \mathrm{~min}$ Date: 1/18/2019 10:30 AM Operator: GM

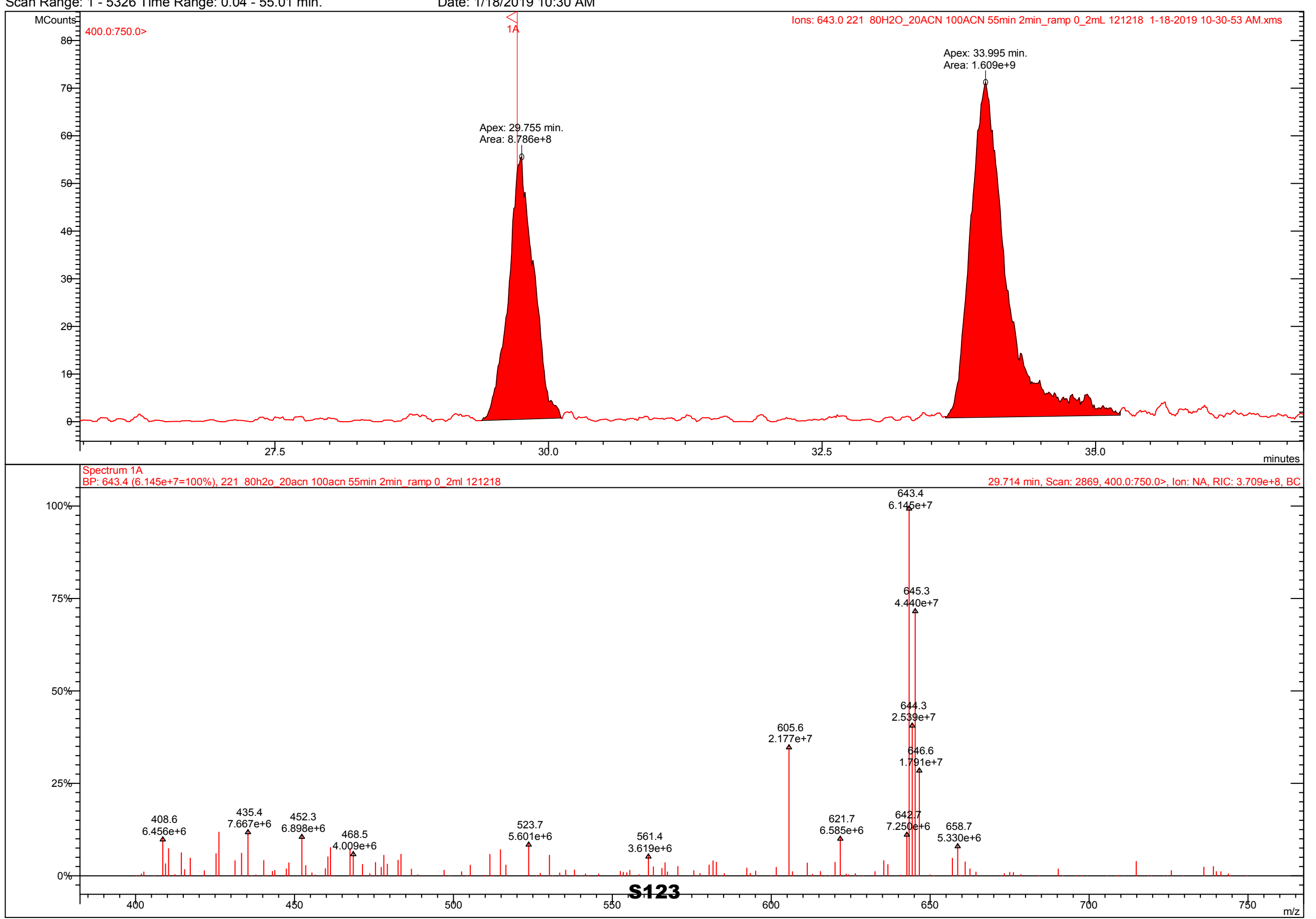


Print Date: 26 Apr 2019 15:49:48 Figure S44. 3-\{6-[5-(4-Bromo-phenyl)-2H-pyrazol-3-yl]-pyridin-2-yl\}-5,6-bis-[4-(3,3-dimethyl-butyl)-phenyl]-[1,2,4]triazine (23)

\section{MS Data Review Active Chromatogram and Spectrum Plots - 4/26/2019 3:48 PM}

File: c:Ittulcarricklgirildatal042519lb-99 80h2o_20acn 100acn 45min 2min_ramp 0_2ml 121218 4-25-2019 11-28-49 am.xms Sample: B-99

Date: 4/25/2019 11:28 AM

Scan Range: 1 - 3812 Time Range: 0.04 - $41.00 \mathrm{~min}$.

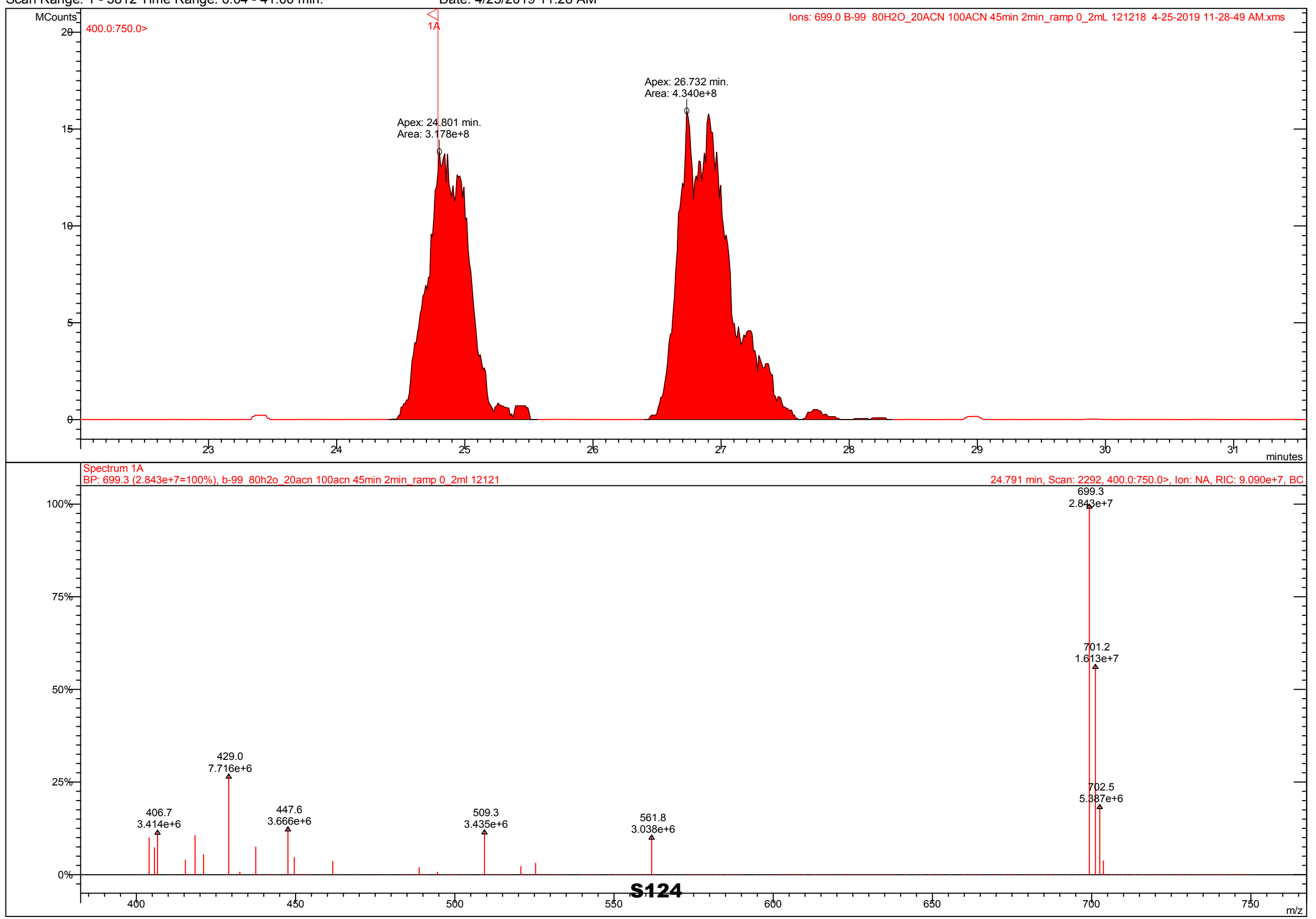




\section{MS Data Review Active Chromatogram and Spectrum Plots - 4/26/2019 3:52 PM}

File: c:Ittulcarricklgirildatal042519lb-97 80h2o_20acn 100acn 45min 2min_ramp 0_2ml 121218 4-25-2019 10-41-47 am.xms Sample: B-97

Scan Range: 1 - 3818 Time Range: 0.02 - $41.01 \mathrm{~min} . \quad$ Date: 4/25/2019 10:41 AM Operator: GM

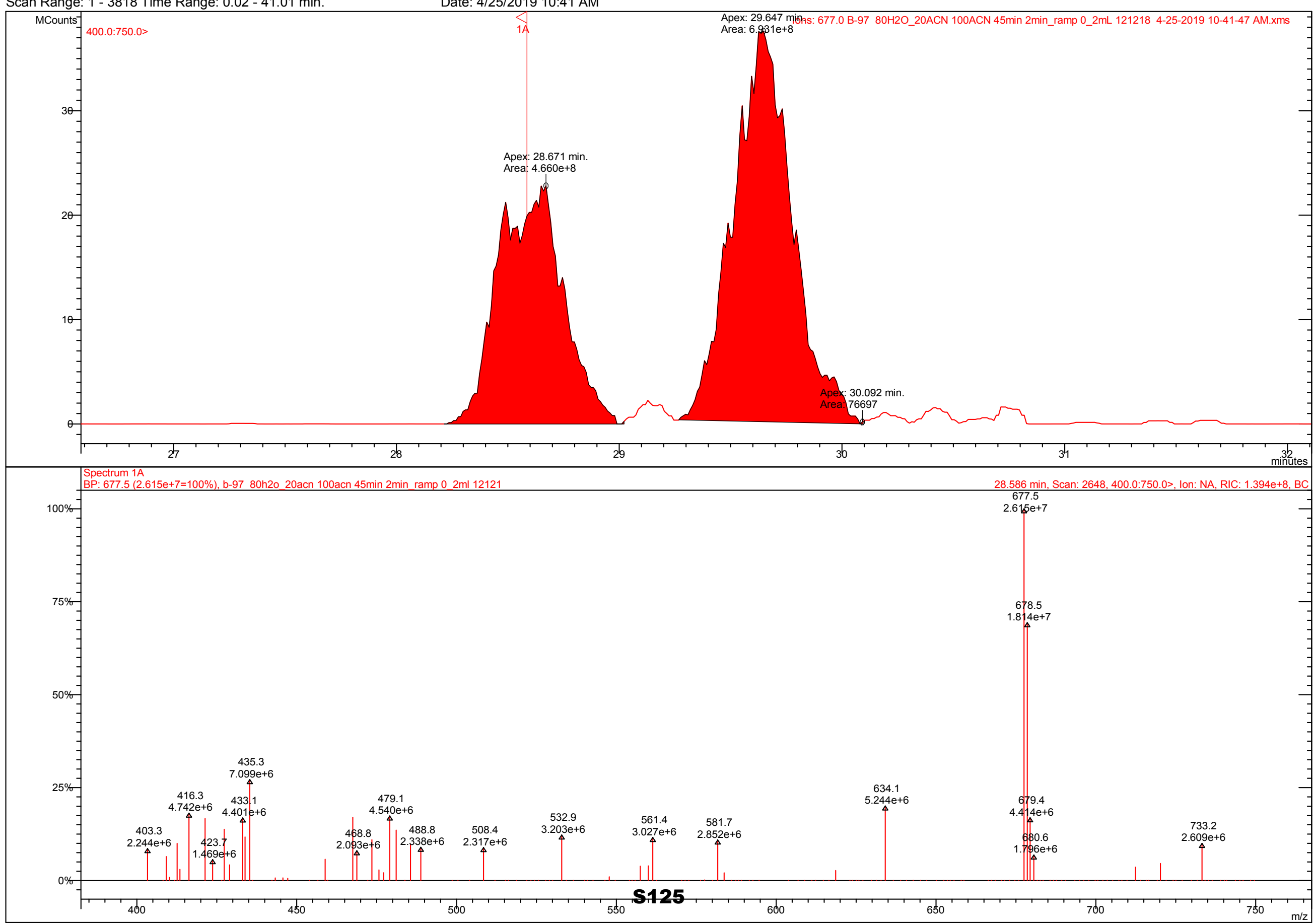


Print Date: 26 Apr 2019 16:00:37 Figure S46. 5,6-Bis-(4-butyl-phenyl)-3-\{6-[5-(3,5-di-tert-butyl-phenyl)-2H-pyrazol-3-yl]-pyridin-2-yl\}-[1,2,4]triazine (25)

\section{MS Data Review Active Chromatogram and Spectrum Plots - 4/26/2019 4:00 PM}

File: c:Ittulcarricklgirildatal042519lb-223 80h2o_20acn 100acn 45min 2min_ramp 0_2ml 121218 4-25-2019 1-49-56 pm.xms Sample: B-223 Operator: GM

Scan Range: 1 - 3826 Time Range: 0.03 - 40.99 min. Date: 4/25/2019 1:49 PM

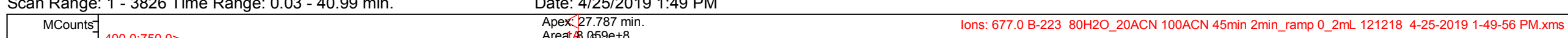

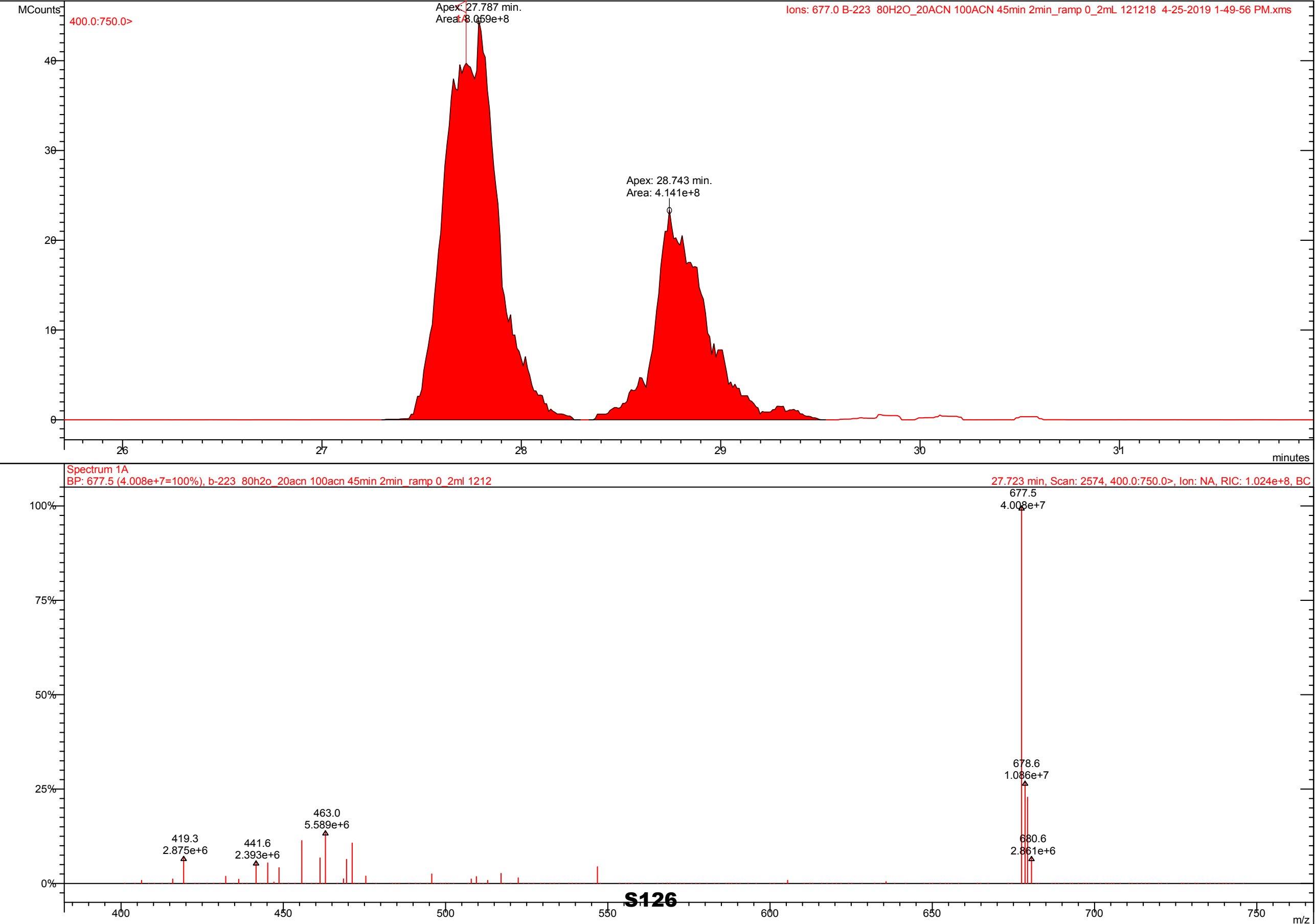


Print Date: 02 Apr 2019 16:23:39 Figure S47. 3-\{6-[5-(4-Bromo-phenyl)-2H-pyrazol-3-yl]-pyridin-2-yl\}-5,6-di-p-tolyl-[1,2,4]triazine (26)

\section{MS Data Review Active Chromatogram and Spectrum Plots - 4/2/2019 4:23 PM}

File: c:Ittulcarricklgirildatal121318l107 80h2o_20acn 100acn 33min curve 0_2ml 121218 12-13-2018 1-45-48 pm.xms Sample: 107

Operator: GM

Scan Range: 1 - 2778 Time Range: 0.03 - 28.99 min

Date: 12/13/2018 1:45 PM

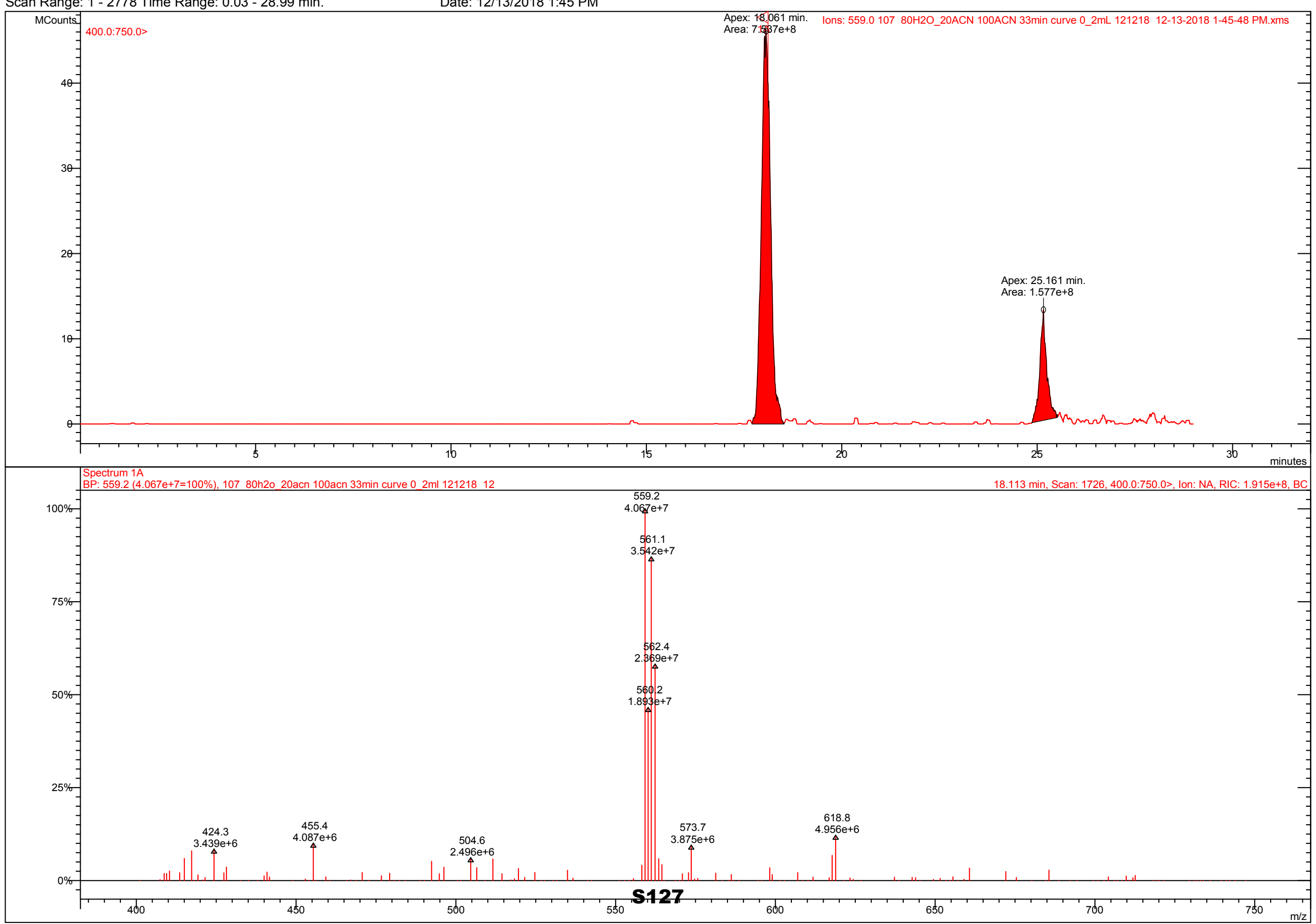


Print Date: 18 Apr 2019 10:29:56 Figure S48. 5,6-Bis-(4-fluoro-phenyl)-3-\{6-[5-(4-methoxy-phenyl)-2H-pyrazol-3-yl]-pyridin-2-yl\}-[1,2,4]triazine (27)

\section{MS Data Review Active Chromatogram and Spectrum Plots - 4/18/2019 10:29 AM}

File: c:Ittulcarricklgirildatal0411519lb-93 80h2o_20acn 100acn 45min 2min_ramp 0_2ml 121218 4-17-2019 8-23-26 am.xms Sample: B-93 Operator: GM

Scan Range: 1 - 3944 Time Range: $0.04-41.00 \mathrm{~min}$

Date: $4 / 17 / 2019$ 8:23 AM

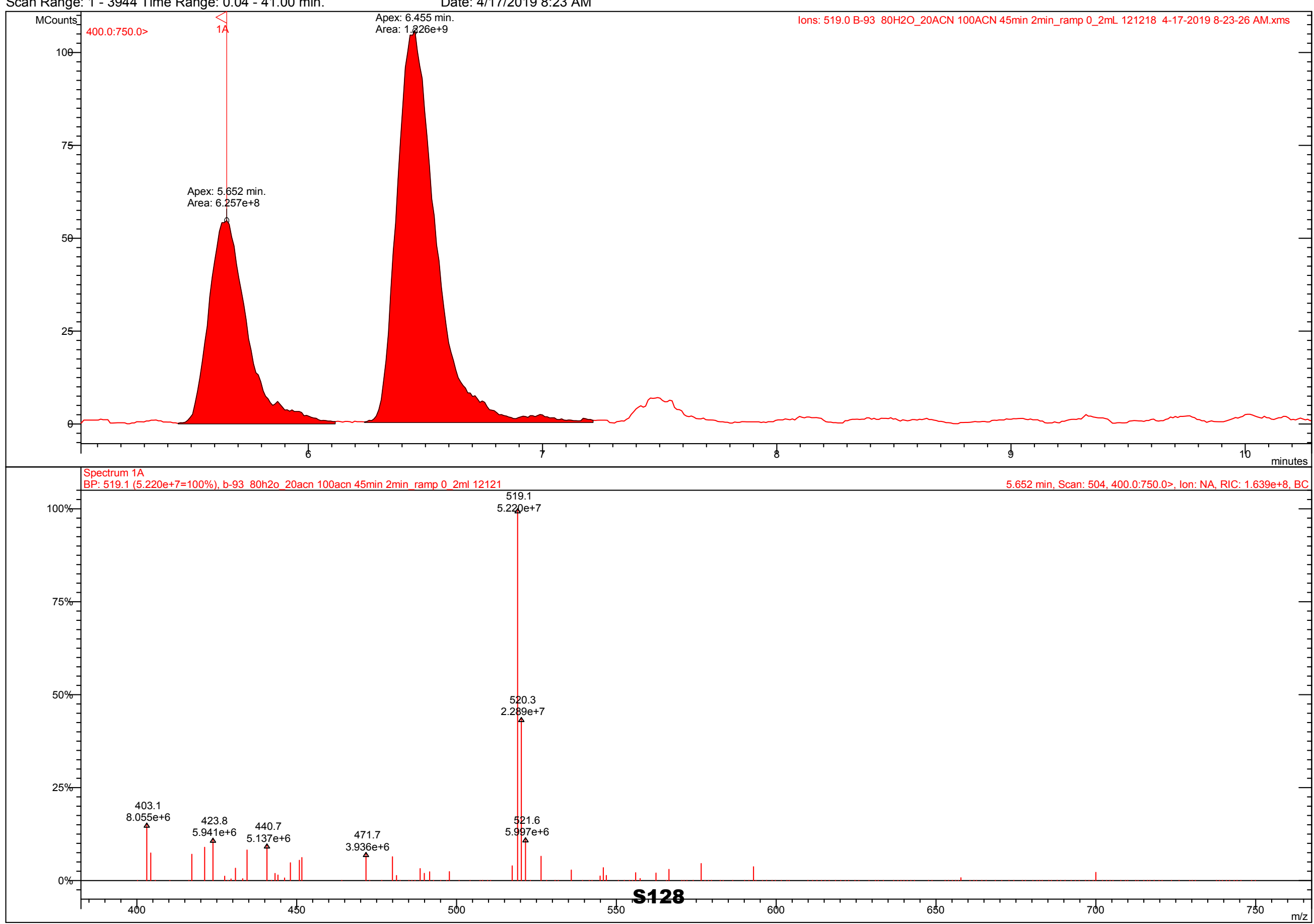


Print Date: 18 Apr 2019 10:23:05 Figure S49. 5,6-Bis-(4-cyclopropyl-phenyl)-3-\{6-[5-(2,4,6-trifluoro-phenyl)-2H-pyrazol-3-yl]-pyridin-2-yl\}-[1,2,4]triazine (28)

\section{MS Data Review Active Chromatogram and Spectrum Plots - 4/18/2019 10:22 AM}

File: c:Ittulcarricklgirildatal0411519lb-277 80h2o_20acn 100acn 45min 2min_ramp 0_2ml 121218 4-17-2019 10-44-31 am.xms Sample: B-277

Operator: GM

Scan Range: 1 - 3968 Time Range: 0.04 - $41.01 \mathrm{~min}$.

Date: 4/17/2019 10:44 AM

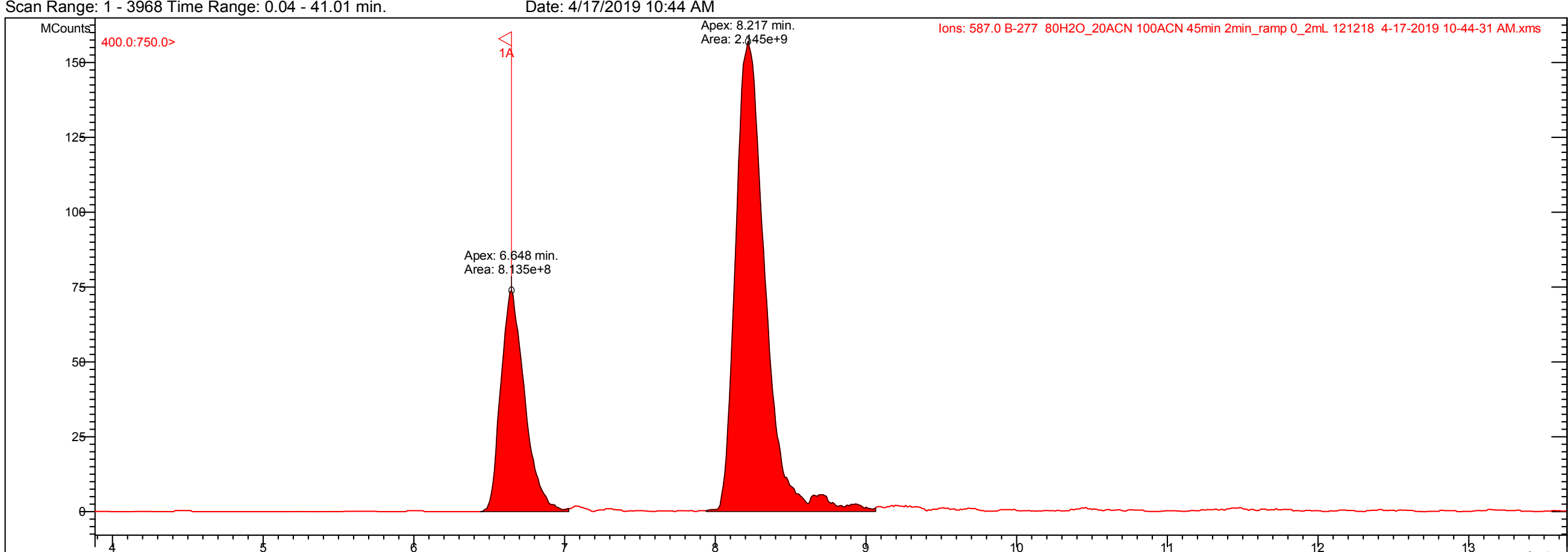

Spectrum $1 \mathrm{~A}$
$\mathrm{BP}: 587.3(6.495 \mathrm{e}+7=100 \%), \ldots .780 \mathrm{~h} 20 \quad 20 \mathrm{acn} 100 \mathrm{acn} 45 \mathrm{~min} 2 \mathrm{~min}$ ramp $02 \mathrm{ml} 12121$

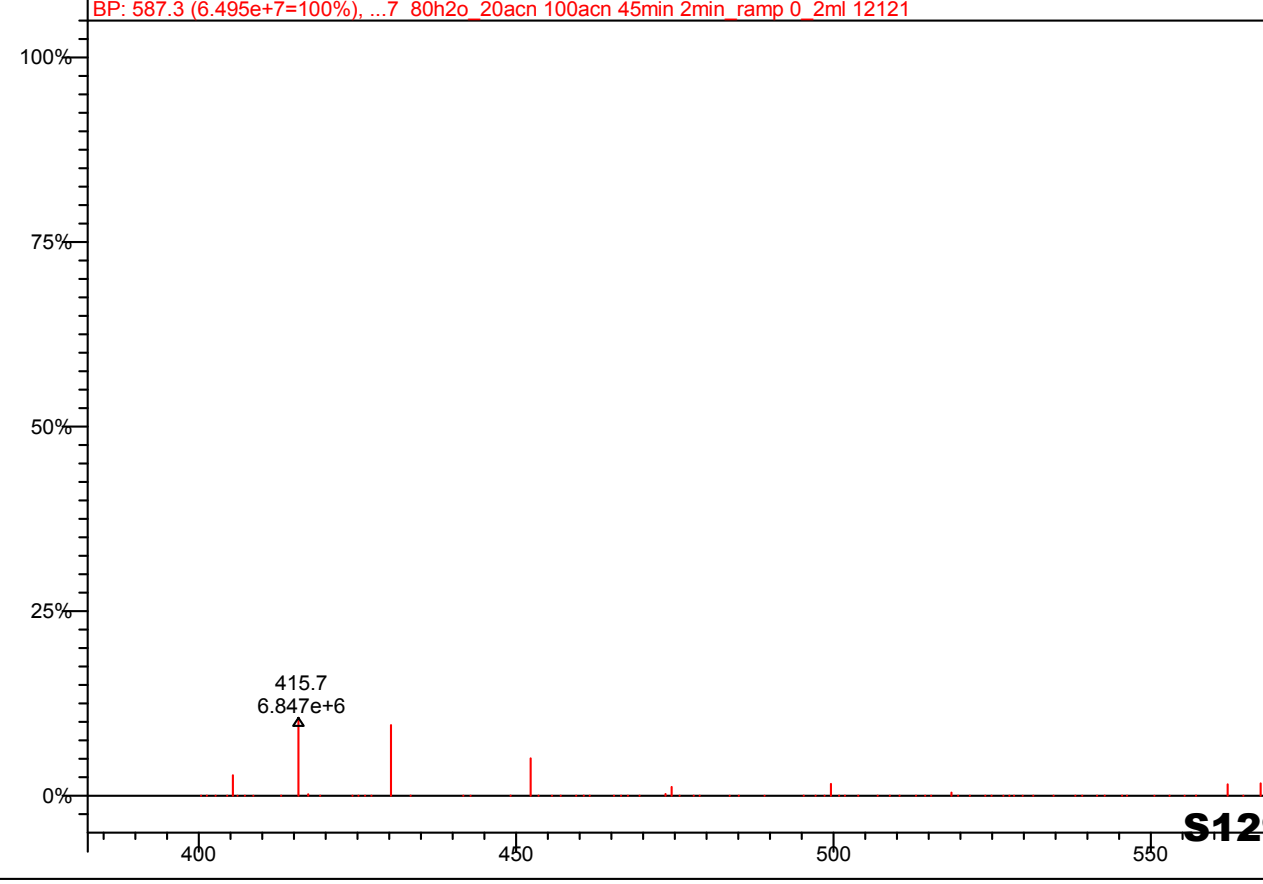

587.3

$6.648 \mathrm{~min}$, Scan: $600,400.0: 750.0>$, Ion: NA, RIC: $1.148 \mathrm{e}+8, \mathrm{BC}$ 

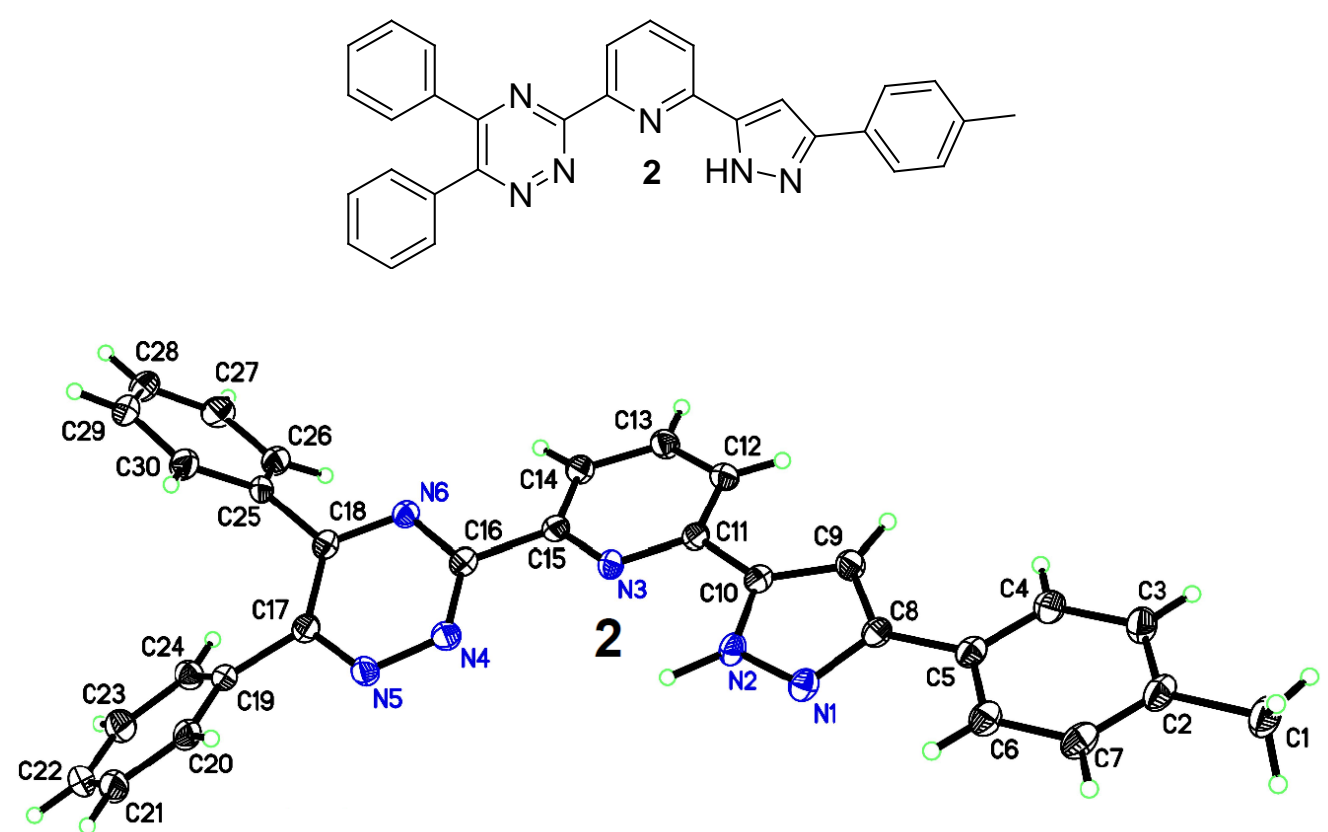

X-ray Structure Report

\author{
for \\ Jesse Carrick \\ Dept. of Chemistry, Tennessee \\ Tech jcarrick@tntech.edu
}




\section{$\underline{\text { DISCUSSION }}$}

The compound, 5,6-diphenyl-3-(6-(3-(p-tolyl)-1H-pyrazol-5-yl)pyridin-2-yl)-1,2,4-triazine, crystallizes as yellow rod-like crystals from an ethanol / THF solution. There are four molecules of the compound, and associated ethanol and THF of crystallization in the unit cell of the primitive, centrosymmetric, monoclinic space group $\mathrm{P} 21 / \mathrm{n}$.

The connectivity of the compound is as proposed (see Figure). The molecules do not display any unexpected derived metrics (see Tables of Bond Distances and Angles). The mean plane of the tolyl ring forms an angle of $7.85(8)^{\circ}$ with respect to the mean plane of the pyrazole ring. The pyridine ring is also nearly co-planar with the pyrazole ring $\left(7.40(7)^{\circ}\right)$ while the triazine ring is synclinial with the pyradine ring at $18.40(6)^{\circ}$. The phenyl rings form periplanar angles of $38.50(5)$ and $56.48(4)^{\circ}$ with respect to the triazine ring mean plane. The triazine shows some distortion about the ring with the largest deviation from the mean plane being $0.052 \AA$ for C11.

There is residual electron density within the lattice located near the THF of crystallization that could not be reliably modeled as any particular solvent. This residual electron density was accounted for using the SQUEEZE routine within PLATON [5]. The contributions from two symmetric void spaces of $252 \AA^{3}$ accounting for 57 electrons each were accounted for.

The hydroxyl and pyrazole $\mathrm{NH}$ hydrogen atoms were freely refined and form discrete hydrogen bonded molecules within the unit cell. Hydrogen bonding does not extend beyond the standard unit. Details of the contacts are presented in the Table of Hydrogen Bonds.

\section{CRYSTAL SUMMARY}

Crystal data for $\mathrm{C}_{36} \mathrm{H}_{36} \mathrm{~N}_{6} \mathrm{O}_{2} ; \mathrm{Mr}_{\mathrm{r}}=584.71$; Monoclinic; space group $\mathrm{P} 21 / \mathrm{n} ; a=11.0293(11) \AA$ $=30.286(3) \AA ; c=11.1297(11) \AA ; \alpha=90^{\circ} ; \beta=114.053(2)^{\circ} ; \gamma=90^{\circ} ; \mathrm{V}=3394.9(6) \AA^{3} ; \mathrm{Z}=4 ; \mathrm{T}$ $=120(2) \mathrm{K} ; \lambda(\mathrm{Mo}-\mathrm{K} \alpha)=0.71073 \AA ; \mu(\mathrm{Mo}-\mathrm{K} \alpha)=0.073 \mathrm{~mm}^{-1} ; \mathrm{d}_{\text {calc }}=1.144 \mathrm{~g} . \mathrm{cm}^{-3} ; 68338$ reflections collected; 8442 unique $\left(\mathrm{R}_{\text {int }}=0.0428\right)$; giving $\mathrm{R}_{1}=0.0493$, $\mathrm{wR}_{2}=0.1239$ for 6964 data with $[\mathrm{I}>2 \sigma(\mathrm{I})]$ and $\mathrm{R}_{1}=0.0606, \mathrm{wR}_{2}=0.1328$ for all 8442 data. Residual electron density $\left(\mathrm{e}^{-} . \AA^{-3}\right)$ $\max / \min : 0.550 /-0.470$.

An arbitrary sphere of data was collected on a yellow rod-like crystal, having approximate dimensions of $0.675 \times 0.406 \times 0.324 \mathrm{~mm}$, on a Bruker APEX-II diffractometer using a combination of $\omega$ - and $\varphi$-scans of $0.5^{\circ}$ [1]. Data were corrected for absorption and polarization effects and analyzed for space group determination [2]. The structure was solved by dual-space methods and expanded routinely [3]. The model was refined by full-matrix least-squares analysis of $\mathrm{F}^{2}$ against all reflections [4]. All non-hydrogen atoms were refined with anisotropic atomic displacement parameters. Unless otherwise noted, hydrogen atoms were included in calculated positions. Atomic displacement parameters for the hydrogens were tied to the equivalent isotropic displacement parameter of the atom to which they are bonded $\left(U_{\text {iso }}(H)=1.5 U_{\text {eq }}(C)\right.$ for methyl, $1.2 U_{\text {eq }}(\mathrm{C})$ for all others). 


\section{REFERENCES}

[1] APEX-3. Bruker AXS. Madison, Wisconsin, USA. 2016.

[2] L. Krause, R. Herbst-Irmer, G. M. Sheldrick, \& D. Stalke. J. Appl. Cryst. 2015 48, 3.

[3] G. M. Sheldrick. Acta Cryst., 2015, A71, 3.

[4] G. M. Sheldrick. Acta Cryst., 2015, C71, 3.

[5] Spek, A. L. Acta Cryst., 2009, D65, 148. 
Table S1. Crystal data and structure refinement for VGB-B-279.

Identification code

Empirical formula

Formula weight

Temperature

Wavelength

Crystal system

Space group

Unit cell dimensions

Volume

Z

Density (calculated)

Absorption coefficient $(\mu)$

$\mathrm{F}(000)$

Crystal color, habit

Crystal size

$\theta$ range for data collection

Index ranges

Reflections collected

Independent reflections

Completeness to $\theta=25.242^{\circ}$

Absorption correction

Max. and min. transmission

Refinement method

Data / restraints / parameters

Goodness-of-fit on $\mathrm{F}^{2}$

Final $R$ indices $[\mathrm{I}>2 \sigma(\mathrm{I})]$

$\mathrm{R}$ indices (all data)

Extinction coefficient

Largest diff. peak and hole
VGB-B-279

$\mathrm{C}_{36} \mathrm{H}_{36} \mathrm{~N}_{6} \mathrm{O}_{2}$

584.71

120(2) K

$0.71073 \AA$

Monoclinic

$\mathrm{P} 21 / \mathrm{n}$

$a=11.0293(11) \AA \quad \alpha=90^{\circ}$

$b=30.286(3) \AA \quad \beta=114.053(2)^{\circ}$

$c=11.1297(11) \AA \quad \gamma=90^{\circ}$

3394.9(6) $\AA^{3}$

4

1.144 g.cm ${ }^{-3}$

$0.073 \mathrm{~mm}^{-1}$

1240

yellow, rod

$0.675 \times 0.406 \times 0.324 \mathrm{~mm}^{3}$

2.114 to $28.320^{\circ}$

$-14 \leq \mathrm{h} \leq 14,-40 \leq \mathrm{k} \leq 40,-14 \leq 1 \leq 14$

68338

$8442\left[\mathrm{R}_{\text {int }}=0.0428\right]$

$100.0 \%$

Numerical

1.0000 and 0.9462

Full-matrix least-squares on $\mathrm{F}^{2}$

8442 / 0 / 407

1.032

$\mathrm{R}_{1}=0.0493, \mathrm{wR}_{2}=0.1239$

$\mathrm{R}_{1}=0.0606, \mathrm{wR}_{2}=0.1328$

$\mathrm{n} / \mathrm{a}$

0.550 and $-0.470 \mathrm{e}^{-} . \AA^{-3}$ 
Table S2. Atomic coordinates and equivalent isotropic displacement parameters $\left(\AA^{2}\right)$ for VGB-B-279.

$\mathrm{U}(\mathrm{eq})$ is defined as one third of the trace of the orthogonalized $\mathrm{U}_{\mathrm{ij}}$ tensor.

\begin{tabular}{|c|c|c|c|c|}
\hline & $\mathrm{x}$ & $\mathrm{y}$ & $\mathrm{z}$ & $\mathrm{U}(\mathrm{eq})$ \\
\hline $\mathrm{N}(1)$ & $0.66736(11)$ & $0.16861(4)$ & $-0.06793(10)$ & $0.021(1)$ \\
\hline $\mathrm{N}(2)$ & $0.71433(11)$ & $0.16647(4)$ & $0.06458(10)$ & $0.020(1)$ \\
\hline $\mathrm{N}(3)$ & $0.82929(10)$ & $0.17143(3)$ & $0.33364(10)$ & $0.019(1)$ \\
\hline $\mathrm{N}(4)$ & $0.74319(11)$ & $0.11195(4)$ & $0.46488(10)$ & $0.022(1)$ \\
\hline $\mathrm{N}(5)$ & $0.69995(11)$ & $0.08292(4)$ & $0.52962(11)$ & $0.022(1)$ \\
\hline $\mathrm{N}(6)$ & $0.91363(10)$ & $0.13612(4)$ & $0.66688(10)$ & $0.020(1)$ \\
\hline $\mathrm{C}(1)$ & $0.72798(12)$ & $0.20379(4)$ & $-0.09201(12)$ & $0.020(1)$ \\
\hline $\mathrm{C}(2)$ & $0.81296(13)$ & $0.22434(4)$ & $0.02593(12)$ & $0.021(1)$ \\
\hline C(3) & $0.80112(12)$ & $0.19938(4)$ & $0.12479(12)$ & $0.019(1)$ \\
\hline C(4) & $0.85813(12)$ & $0.20408(4)$ & $0.26782(12)$ & $0.019(1)$ \\
\hline$C(5)$ & $0.93320(13)$ & $0.24104(4)$ & $0.32966(13)$ & $0.022(1)$ \\
\hline C(6) & 0.98187(13) & $0.24397(4)$ & $0.46524(13)$ & $0.024(1)$ \\
\hline C(7) & $0.95545(13)$ & $0.21016(4)$ & $0.53487(12)$ & $0.022(1)$ \\
\hline $\mathrm{C}(8)$ & $0.87801(12)$ & $0.17485(4)$ & $0.46498(12)$ & $0.019(1)$ \\
\hline C(9) & $0.84215(12)$ & $0.13900(4)$ & $0.53606(12)$ & $0.019(1)$ \\
\hline$C(10)$ & $0.76139(12)$ & $0.07992(4)$ & $0.66085(12)$ & $0.019(1)$ \\
\hline $\mathrm{C}(11)$ & $0.87785(12)$ & $0.10517(4)$ & $0.73005(12)$ & $0.019(1)$ \\
\hline $\mathrm{C}(12)$ & $0.69988(12)$ & $0.21643(4)$ & $-0.22850(12)$ & $0.020(1)$ \\
\hline C(13) & $0.74686(14)$ & $0.25611(5)$ & $-0.25659(13)$ & $0.024(1)$ \\
\hline $\mathrm{C}(14)$ & $0.71901(14)$ & $0.26791(5)$ & $-0.38553(14)$ & $0.026(1)$ \\
\hline$C(15)$ & $0.64205(13)$ & $0.24100(5)$ & $-0.49037(13)$ & $0.025(1)$ \\
\hline$C(16)$ & $0.59431(14)$ & $0.20148(5)$ & $-0.46231(13)$ & $0.025(1)$ \\
\hline $\mathrm{C}(17)$ & $0.62315(13)$ & $0.18917(5)$ & $-0.33354(13)$ & $0.023(1)$ \\
\hline C(18) & $0.60969(15)$ & $0.25472(5)$ & $-0.63014(14)$ & 0.031(1) \\
\hline C(19) & $0.69602(12)$ & $0.05044(4)$ & $0.72298(12)$ & $0.020(1)$ \\
\hline$C(20)$ & $0.63209(14)$ & $0.01215(4)$ & $0.65783(13)$ & $0.025(1)$ \\
\hline $\mathrm{C}(21)$ & $0.56113(15)$ & $-0.01378(5)$ & $0.70966(14)$ & $0.031(1)$ \\
\hline $\mathrm{C}(22)$ & $0.55287(14)$ & $-0.00176(5)$ & $0.82596(15)$ & $0.032(1)$ \\
\hline C(23) & $0.61564(14)$ & $0.03633(5)$ & $0.89118(15)$ & $0.029(1)$ \\
\hline$C(24)$ & $0.68742(13)$ & $0.06233(4)$ & $0.84031(13)$ & $0.024(1)$ \\
\hline$C(25)$ & $0.96527(12)$ & $0.09820(4)$ & $0.87088(12)$ & $0.020(1)$ \\
\hline$C(26)$ & $0.99624(13)$ & $0.13315(4)$ & $0.95948(13)$ & $0.023(1)$ \\
\hline $\mathrm{C}(27)$ & $1.07815(14)$ & $0.12619(5)$ & $1.09134(13)$ & $0.028(1)$ \\
\hline C(28) & $1.13322(14)$ & $0.08497(5)$ & $1.13324(14)$ & $0.031(1)$ \\
\hline C(29) & $1.10521(14)$ & $0.05037(5)$ & $1.04504(15)$ & $0.032(1)$ \\
\hline C(30) & $1.01929(14)$ & $0.05655(4)$ & $0.91433(14)$ & $0.026(1)$ \\
\hline $\mathrm{O}(2 \mathrm{~S})$ & $1.30468(13)$ & $0.13756(5)$ & $0.44095(13)$ & $0.047(1)$ \\
\hline C(3S) & $1.19804(19)$ & $0.15034(6)$ & $0.4727(2)$ & $0.046(1)$ \\
\hline $\mathrm{C}(4 \mathrm{~S})$ & $1.2377(3)$ & $0.14083(10)$ & $0.6142(2)$ & $0.070(1)$ \\
\hline
\end{tabular}




\begin{tabular}{|c|c|c|c|c|}
\hline$C(5 S)$ & $1.34424(19)$ & $0.10556(7)$ & $0.6482(2)$ & $0.049(1)$ \\
\hline $\mathrm{C}(6 \mathrm{~S})$ & 1.36029(19) & $0.09928(7)$ & $0.51954(19)$ & $0.049(1)$ \\
\hline $\mathrm{O}(1 \mathrm{~S})$ & $0.59261(10)$ & $0.10664(3)$ & $0.17800(10)$ & $0.028(1)$ \\
\hline $\mathrm{C}(1 \mathrm{~S})$ & $0.46675(15)$ & $0.12703(5)$ & $0.14789(15)$ & $0.032(1)$ \\
\hline $\mathrm{C}(2 \mathrm{~S})$ & $0.38187(16)$ & $0.12093(6)$ & $0.00341(15)$ & $0.036(1)$ \\
\hline $\mathrm{H}(2 \mathrm{~N})$ & $0.6832(19)$ & $0.1451(7)$ & $0.1041(19)$ & $0.042(5)$ \\
\hline $\mathrm{H}(2)$ & 0.86548 & 0.24922 & 0.03530 & 0.025 \\
\hline $\mathrm{H}(5)$ & 0.95012 & 0.26322 & 0.28076 & 0.026 \\
\hline $\mathrm{H}(6)$ & 1.03167 & 0.26832 & 0.50906 & 0.028 \\
\hline $\mathrm{H}(7)$ & 0.98869 & 0.21103 & 0.62622 & 0.027 \\
\hline $\mathrm{H}(13)$ & 0.79734 & 0.27483 & -0.18825 & 0.029 \\
\hline $\mathrm{H}(14)$ & 0.75236 & 0.29429 & -0.40232 & 0.032 \\
\hline $\mathrm{H}(16)$ & 0.54237 & 0.18309 & -0.53093 & 0.030 \\
\hline $\mathrm{H}(17)$ & 0.59114 & 0.16251 & -0.31680 & 0.028 \\
\hline $\mathrm{H}(18 \mathrm{~A})$ & 0.59719 & 0.22891 & -0.68397 & 0.046 \\
\hline $\mathrm{H}(18 \mathrm{~B})$ & 0.68146 & 0.27198 & -0.63302 & 0.046 \\
\hline $\mathrm{H}(18 \mathrm{C})$ & 0.52981 & 0.27201 & -0.66254 & 0.046 \\
\hline $\mathrm{H}(20)$ & 0.63705 & 0.00397 & 0.57942 & 0.030 \\
\hline $\mathrm{H}(21)$ & 0.51904 & -0.03930 & 0.66602 & 0.037 \\
\hline $\mathrm{H}(22)$ & 0.50532 & -0.01918 & 0.86046 & 0.038 \\
\hline $\mathrm{H}(23)$ & 0.60964 & 0.04448 & 0.96914 & 0.035 \\
\hline $\mathrm{H}(24)$ & 0.72981 & 0.08771 & 0.88464 & 0.028 \\
\hline $\mathrm{H}(26)$ & 0.96220 & 0.16114 & 0.93056 & 0.027 \\
\hline $\mathrm{H}(27)$ & 1.09591 & 0.14923 & 1.15134 & 0.034 \\
\hline $\mathrm{H}(28)$ & 1.18935 & 0.08053 & 1.22115 & 0.037 \\
\hline H(29) & 1.14400 & 0.02289 & 1.07333 & 0.038 \\
\hline $\mathrm{H}(30)$ & 0.99776 & 0.03297 & 0.85581 & 0.032 \\
\hline H(3SA) & 1.11862 & 0.13390 & 0.41998 & 0.055 \\
\hline H(3SB) & 1.17972 & 0.18158 & 0.45562 & 0.055 \\
\hline $\mathrm{H}(4 \mathrm{SA})$ & 1.16249 & 0.13017 & 0.62995 & 0.084 \\
\hline $\mathrm{H}(4 \mathrm{SB})$ & 1.27240 & 0.16716 & 0.66667 & 0.084 \\
\hline $\mathrm{H}(5 \mathrm{SA})$ & 1.31600 & 0.07840 & 0.67526 & 0.058 \\
\hline $\mathrm{H}(5 \mathrm{SB})$ & 1.42656 & 0.11541 & 0.71792 & 0.058 \\
\hline H(6SA) & 1.45344 & 0.09638 & 0.53646 & 0.059 \\
\hline $\mathrm{H}(6 \mathrm{SB})$ & 1.31400 & 0.07289 & 0.47466 & 0.059 \\
\hline $\mathrm{H}(1 \mathrm{OA})$ & $0.640(2)$ & $0.1105(7)$ & $0.261(2)$ & $0.046(6)$ \\
\hline $\mathrm{H}(1 \mathrm{SA})$ & 0.47868 & 0.15825 & 0.16895 & 0.039 \\
\hline $\mathrm{H}(1 \mathrm{SB})$ & 0.42395 & 0.11364 & 0.19965 & 0.039 \\
\hline $\mathrm{H}(2 \mathrm{SA})$ & 0.42407 & 0.13474 & -0.04714 & 0.055 \\
\hline $\mathrm{H}(2 \mathrm{SB})$ & 0.29650 & 0.13418 & -0.01798 & 0.055 \\
\hline $\mathrm{H}(2 \mathrm{SC})$ & 0.37097 & 0.08997 & -0.01674 & 0.055 \\
\hline
\end{tabular}


Table S3. Anisotropic displacement parameters $\left(\AA^{2}\right)$ for VGB-B-279.

The anisotropic displacement factor exponent takes the form:

$-2 \pi^{2}\left[h^{2} a^{2} U_{11}+\ldots+2 h k a * b * U_{12}\right]$

$$
\begin{array}{llllll}
\mathrm{U}_{11} & \mathrm{U}_{22} & \mathrm{U}_{33} & \mathrm{U}_{23} & \mathrm{U}_{13} & \mathrm{U}_{12}
\end{array}
$$

\begin{tabular}{|c|c|c|c|c|c|c|}
\hline 1) & (5) & 5) & (5) & (4) & (4) & 4) \\
\hline (2) & $0.0240(5)$ & $0.0200(5)$ & $0.0154(5)$ & $0.0010(4)$ & $0.0063(4)$ & $-0.0006(4)$ \\
\hline (3) & $0190(5)$ & 0202(5) & $0.0165(5)$ & $0.0006(4)$ & $0.0060(4)$ & (1) \\
\hline (4) & $227(5)$ & 24(5) & $0.0182(5)$ & 0001(4) & 0064(4) & $0035(4)$ \\
\hline (5) & (5) & 5) & 0.01 & $.0016(4)$ & $067(4)$ & -0.0030( \\
\hline (6) & 191(5) & 0207(5) & & & $0.0056(4)$ & $-c$ \\
\hline & 6) & $0208(6)$ & 0.01 & -0.00 & & \\
\hline 2) & & & & $-0.0001(5)$ & & \\
\hline 3) & $0183(6)$ & $03(6)$ & $32(6)$ & $0.0005(4)$ & & $0.0006(4)$ \\
\hline (4) & $0164(5)$ & $.0221(6)$ & $0.0176(6)$ & $0.0014(5)$ & 0064(5) & $0.0005(4$ \\
\hline (5) & 6) & 6) & 0.0 & & 0.0088(5) & $-0.0038(5)$ \\
\hline 6) & & 6) & & 5) & & \\
\hline & & 5) & & -0.0 & & \\
\hline (8) & 6) & 6) & & & (5) & 01(4) \\
\hline (9) & & & & & & 9(4) \\
\hline (10) & & ) & & & & (4) \\
\hline 11) & & ) & & 4) & (5) & 6(4) \\
\hline & & & & & & \\
\hline (13) & & & & & 87(5) & 08(5) \\
\hline & & 7) & & & & \\
\hline r & & 7) & & & (5) & (5) \\
\hline (16 & & & & -0.0 & & 7(5) \\
\hline (17) & (6) & 0.0 & & -0.0 & 82(5) & 024(5) \\
\hline C(18) & & & & & & \\
\hline 1) & & ) & & & (5) & (4) \\
\hline$(2$ & & )) & & 0.0 & $0.0024(5)$ & $6(5)$ \\
\hline C(21) & & 7) & & & $0.0022(6)$ & $0(5)$ \\
\hline $\mathrm{C}(22)$ & & & & & 099(6) & $38(6)$ \\
\hline & & & & & & \\
\hline & & ) & & & (5) & $5(5)$ \\
\hline C(25) & & & & & $0.0057(5)$ & $13(4)$ \\
\hline $\mathrm{C}(26)$ & (6) & 0.02 & 0.02 & 0.00 & 0.0071(5) & $0.0000(5)$ \\
\hline C(27) & & & & & 0.0056(5) & 61(6) \\
\hline & & & & & & \\
\hline & & & & & $0.0033(6)$ & 30 \\
\hline C(30) & & 0.0 & & & 0.0051(5) & $-0.0006(5)$ \\
\hline $\mathrm{O}(2 \mathrm{~S})$ & 0399(7) & $0.0569(8)$ & $0.0431(7)$ & $0.0012(6)$ & $0.0175(6)$ & $0.0072(6)$ \\
\hline & 0423(9) & (10) & 11) & & $0.0233(9)$ & \\
\hline & 0685(15) & 0972(19) & $0.0517(13)$ & ) & 0021501 & \\
\hline
\end{tabular}


$\begin{array}{lllllll}\mathrm{C}(5 \mathrm{~S}) & 0.0380(9) & 0.0540(11) & 0.0493(11) & 0.0107(9) & 0.0129(8) & 0.0074(8)\end{array}$

$\begin{array}{llllllll}\mathrm{C}(6 \mathrm{~S}) & 0.0365(9) & 0.0518(11) & 0.0482(10) & -0.0113(8) & 0.0060(8) & 0.0127(8)\end{array}$

$\begin{array}{lllllll}\mathrm{O}(1 \mathrm{~S}) & 0.0258(5) & 0.0299(5) & 0.0222(5) & 0.0016(4) & 0.0049(4) & -0.0048(4)\end{array}$

$\begin{array}{lllllll}\mathrm{C}(1 \mathrm{~S}) & 0.0317(7) & 0.0371(8) & 0.0268(7) & 0.0004(6) & 0.0101(6) & -0.0005(6)\end{array}$

$\begin{array}{lllllll}\mathrm{C}(2 \mathrm{~S}) & 0.0284(7) & 0.0460(9) & 0.0290(8) & 0.0023(7) & 0.0059(6) & 0.0004(7)\end{array}$ 
Table S4. Bond lengths [Å] for VGB-B-279.

$\begin{array}{llll}\text { atom-atom } & \text { distance } & \text { atom-atom } & \text { distance } \\ \mathrm{N}(1)-\mathrm{C}(1) & & & \\ \mathrm{N}(2)-\mathrm{C}(3) & 1.3419(17) & \mathrm{N}(1)-\mathrm{N}(2) & 1.3508(15) \\ \mathrm{N}(3)-\mathrm{C}(4) & 1.3548(16) & \mathrm{N}(3)-\mathrm{C}(8) & 1.3401(16) \\ \mathrm{N}(4)-\mathrm{N}(5) & 1.3437(16) & \mathrm{N}(4)-\mathrm{C}(9) & 1.3361(16) \\ \mathrm{N}(6)-\mathrm{C}(11) & 1.3414(15) & \mathrm{N}(5)-\mathrm{C}(10) & 1.3394(16) \\ \mathrm{C}(1)-\mathrm{C}(2) & 1.3248(16) & \mathrm{N}(6)-\mathrm{C}(9) & 1.3466(16) \\ \mathrm{C}(2)-\mathrm{C}(3) & 1.4093(17) & \mathrm{C}(1)-\mathrm{C}(12) & 1.4719(17) \\ \mathrm{C}(4)-\mathrm{C}(5) & 1.3843(17) & \mathrm{C}(3)-\mathrm{C}(4) & 1.4608(17) \\ \mathrm{C}(6)-\mathrm{C}(7) & 1.3956(18) & \mathrm{C}(5)-\mathrm{C}(6) & 1.3830(18) \\ \mathrm{C}(8)-\mathrm{C}(9) & 1.3851(18) & \mathrm{C}(7)-\mathrm{C}(8) & 1.3916(18) \\ \mathrm{C}(10)-\mathrm{C}(19) & 1.4887(17) & \mathrm{C}(10)-\mathrm{C}(11) & 1.4217(17) \\ \mathrm{C}(12)-\mathrm{C}(13) & 1.4836(17) & \mathrm{C}(11)-\mathrm{C}(25) & 1.4829(17) \\ \mathrm{C}(13)-\mathrm{C}(14) & 1.3937(19) & \mathrm{C}(12)-\mathrm{C}(17) & 1.3993(18) \\ \mathrm{C}(15)-\mathrm{C}(16) & 1.3864(19) & \mathrm{C}(14)-\mathrm{C}(15) & 1.392(2) \\ \mathrm{C}(16)-\mathrm{C}(17) & 1.393(2) & \mathrm{C}(15)-\mathrm{C}(18) & 1.5057(18) \\ \mathrm{C}(19)-\mathrm{C}(20) & 1.3866(18) & \mathrm{C}(19)-\mathrm{C}(24) & 1.3946(18) \\ \mathrm{C}(21)-\mathrm{C}(22) & 1.3969(18) & \mathrm{C}(20)-\mathrm{C}(21) & 1.3893(19) \\ \mathrm{C}(23)-\mathrm{C}(24) & 1.383(2) & \mathrm{C}(22)-\mathrm{C}(23) & 1.388(2) \\ \mathrm{C}(25)-\mathrm{C}(30) & 1.3902(19) & \mathrm{C}(25)-\mathrm{C}(26) & 1.3910(18) \\ \mathrm{C}(27)-\mathrm{C}(28) & 1.3946(18) & \mathrm{C}(26)-\mathrm{C}(27) & 1.3905(19) \\ \mathrm{C}(29)-\mathrm{C}(30) & 1.383(2) & \mathrm{C}(28)-\mathrm{C}(29) & 1.382(2) \\ \mathrm{O}(2 \mathrm{~S})-\mathrm{C}(6 \mathrm{~S}) & 1.388(2) & \mathrm{O}(2 \mathrm{~S})-\mathrm{C}(3 \mathrm{~S}) & 1.413(2) \\ \mathrm{C}(4 \mathrm{~S})-\mathrm{C}(5 S) & 1.431(2) & \mathrm{C}(3 \mathrm{~S})-\mathrm{C}(4 \mathrm{~S}) & 1.481(3) \\ \mathrm{O}(1 \mathrm{~S})-\mathrm{C}(1 \mathrm{~S}) & 1.517(3) & \mathrm{C}(5 \mathrm{~S})-\mathrm{C}(6 \mathrm{~S}) & 1.525(3) \\ & 1.4288(19) & \mathrm{C}(1 \mathrm{~S})-\mathrm{C}(2 \mathrm{~S}) & 1.506(2)\end{array}$

Symmetry transformations used to generate equivalent atoms: 
Table S5. Bond angles [ $\left.{ }^{\circ}\right]$ for VGB-B-279.

\begin{tabular}{|c|c|c|c|}
\hline atom-atom-atom & angle & atom-atom-atom & angle \\
\hline $\mathrm{C}(1)-\mathrm{N}(1)-\mathrm{N}(2)$ & 104.80(10) & $\mathrm{N}(1)-\mathrm{N}(2)-\mathrm{C}(3)$ & $112.58(10)$ \\
\hline $\mathrm{C}(8)-\mathrm{N}(3)-\mathrm{C}(4)$ & 117.32(11) & $\mathrm{C}(9)-\mathrm{N}(4)-\mathrm{N}(5)$ & $117.82(11)$ \\
\hline $\mathrm{C}(10)-\mathrm{N}(5)-\mathrm{N}(4)$ & $119.85(11)$ & $\mathrm{C}(11)-\mathrm{N}(6)-\mathrm{C}(9)$ & $116.73(11)$ \\
\hline $\mathrm{N}(1)-\mathrm{C}(1)-\mathrm{C}(2)$ & $111.16(11)$ & $\mathrm{N}(1)-\mathrm{C}(1)-\mathrm{C}(12)$ & $120.02(11)$ \\
\hline $\mathrm{C}(2)-\mathrm{C}(1)-\mathrm{C}(12)$ & $128.82(12)$ & $\mathrm{C}(3)-\mathrm{C}(2)-\mathrm{C}(1)$ & 104.88(11) \\
\hline $\mathrm{N}(2)-\mathrm{C}(3)-\mathrm{C}(2)$ & 106.57(11) & $\mathrm{N}(2)-\mathrm{C}(3)-\mathrm{C}(4)$ & 120.94(11) \\
\hline $\mathrm{C}(2)-\mathrm{C}(3)-\mathrm{C}(4)$ & $132.44(12)$ & $\mathrm{N}(3)-\mathrm{C}(4)-\mathrm{C}(5)$ & $123.10(11)$ \\
\hline $\mathrm{N}(3)-\mathrm{C}(4)-\mathrm{C}(3)$ & 115.47(11) & $C(5)-C(4)-C(3)$ & $121.39(11)$ \\
\hline$C(6)-C(5)-C(4)$ & $118.55(12)$ & $C(5)-C(6)-C(7)$ & $119.10(12)$ \\
\hline$C(6)-C(7)-C(8)$ & $118.46(12)$ & $\mathrm{N}(3)-\mathrm{C}(8)-\mathrm{C}(7)$ & $123.43(11)$ \\
\hline $\mathrm{N}(3)-\mathrm{C}(8)-\mathrm{C}(9)$ & 116.61(11) & $\mathrm{C}(7)-\mathrm{C}(8)-\mathrm{C}(9)$ & 119.93(11) \\
\hline $\mathrm{N}(4)-\mathrm{C}(9)-\mathrm{N}(6)$ & 125.33(11) & $\mathrm{N}(4)-\mathrm{C}(9)-\mathrm{C}(8)$ & $117.51(11)$ \\
\hline $\mathrm{N}(6)-\mathrm{C}(9)-\mathrm{C}(8)$ & $117.16(11)$ & $N(5)-C(10)-C(11)$ & 119.86(11) \\
\hline $\mathrm{N}(5)-\mathrm{C}(10)-\mathrm{C}(19)$ & $115.03(11)$ & $\mathrm{C}(11)-\mathrm{C}(10)-\mathrm{C}(19)$ & $125.07(11)$ \\
\hline $\mathrm{N}(6)-\mathrm{C}(11)-\mathrm{C}(10)$ & 119.47(11) & $\mathrm{N}(6)-\mathrm{C}(11)-\mathrm{C}(25)$ & $117.10(11)$ \\
\hline$C(10)-C(11)-C(25)$ & $123.41(11)$ & $\mathrm{C}(13)-\mathrm{C}(12)-\mathrm{C}(17)$ & $118.35(12)$ \\
\hline $\mathrm{C}(13)-\mathrm{C}(12)-\mathrm{C}(1)$ & $120.98(12)$ & $\mathrm{C}(17)-\mathrm{C}(12)-\mathrm{C}(1)$ & $120.67(12)$ \\
\hline $\mathrm{C}(14)-\mathrm{C}(13)-\mathrm{C}(12)$ & $120.58(13)$ & C(13)-C(14)-C(15) & $121.25(13)$ \\
\hline $\mathrm{C}(14)-\mathrm{C}(15)-\mathrm{C}(16)$ & $118.16(12)$ & C(14)-C(15)-C(18) & 120.74(13) \\
\hline $\mathrm{C}(16)-\mathrm{C}(15)-\mathrm{C}(18)$ & 121.10(13) & $\mathrm{C}(17)-\mathrm{C}(16)-\mathrm{C}(15)$ & 120.94(13) \\
\hline $\mathrm{C}(16)-\mathrm{C}(17)-\mathrm{C}(12)$ & $120.72(13)$ & C(24)-C(19)-C(20) & 119.19(12) \\
\hline $\mathrm{C}(24)-\mathrm{C}(19)-\mathrm{C}(10)$ & $120.84(11)$ & C(20)-C(19)-C(10) & 119.74(12) \\
\hline C(21)-C(20)-C(19) & $120.29(13)$ & C(22)-C(21)-C(20) & $120.20(13)$ \\
\hline $\mathrm{C}(21)-\mathrm{C}(22)-\mathrm{C}(23)$ & 119.94(13) & C(22)-C(23)-C(24) & $120.20(14)$ \\
\hline $\mathrm{C}(23)-\mathrm{C}(24)-\mathrm{C}(19)$ & 120.17(13) & $\mathrm{C}(26)-\mathrm{C}(25)-\mathrm{C}(30)$ & 119.71(12) \\
\hline $\mathrm{C}(26)-\mathrm{C}(25)-\mathrm{C}(11)$ & $120.47(11)$ & $\mathrm{C}(30)-\mathrm{C}(25)-\mathrm{C}(11)$ & 119.81(12) \\
\hline $\mathrm{C}(27)-\mathrm{C}(26)-\mathrm{C}(25)$ & 119.94(13) & C(28)-C(27)-C(26) & 119.98(13) \\
\hline $\mathrm{C}(29)-\mathrm{C}(28)-\mathrm{C}(27)$ & $120.30(13)$ & C(28)-C(29)-C(30) & 120.11(13) \\
\hline $\mathrm{C}(29)-\mathrm{C}(30)-\mathrm{C}(25)$ & 119.89(13) & $\mathrm{C}(3 \mathrm{~S})-\mathrm{O}(2 \mathrm{~S})-\mathrm{C}(6 \mathrm{~S})$ & $105.20(14)$ \\
\hline $\mathrm{O}(2 \mathrm{~S})-\mathrm{C}(3 \mathrm{~S})-\mathrm{C}(4 \mathrm{~S})$ & 107.32(16) & $\mathrm{C}(3 \mathrm{~S})-\mathrm{C}(4 \mathrm{~S})-\mathrm{C}(5 \mathrm{~S})$ & $105.37(17)$ \\
\hline $\mathrm{C}(4 \mathrm{~S})-\mathrm{C}(5 \mathrm{~S})-\mathrm{C}(6 \mathrm{~S})$ & 103.20(15) & $\mathrm{O}(2 \mathrm{~S})-\mathrm{C}(6 \mathrm{~S})-\mathrm{C}(5 \mathrm{~S})$ & $106.73(14)$ \\
\hline $\mathrm{O}(1 \mathrm{~S})-\mathrm{C}(1 \mathrm{~S})-\mathrm{C}(2 \mathrm{~S})$ & 108.60(13) & & \\
\hline
\end{tabular}

Symmetry transformations used to generate equivalent atoms: 
Table S6. Torsion angles $\left[{ }^{\circ}\right]$ for VGB-B-279.

\begin{tabular}{|c|c|c|c|}
\hline atom-atom-atom-atom & angle & atom-atom-atom-atom & angle \\
\hline $\mathrm{C}(1)-\mathrm{N}(1)-\mathrm{N}(2)-\mathrm{C}(3)$ & $0.77(14)$ & $\mathrm{C}(9)-\mathrm{N}(4)-\mathrm{N}(5)-\mathrm{C}(10)$ & $-4.18(18)$ \\
\hline $\mathrm{N}(2)-\mathrm{N}(1)-\mathrm{C}(1)-\mathrm{C}(2)$ & $-0.56(14)$ & $\mathrm{N}(2)-\mathrm{N}(1)-\mathrm{C}(1)-\mathrm{C}(12)$ & $-179.80(11)$ \\
\hline $\mathrm{N}(1)-\mathrm{C}(1)-\mathrm{C}(2)-\mathrm{C}(3)$ & $0.18(14)$ & $\mathrm{C}(12)-\mathrm{C}(1)-\mathrm{C}(2)-\mathrm{C}(3)$ & 179.34(12) \\
\hline $\mathrm{N}(1)-\mathrm{N}(2)-\mathrm{C}(3)-\mathrm{C}(2)$ & $-0.67(14)$ & $\mathrm{N}(1)-\mathrm{N}(2)-\mathrm{C}(3)-\mathrm{C}(4)$ & $177.02(11)$ \\
\hline $\mathrm{C}(1)-\mathrm{C}(2)-\mathrm{C}(3)-\mathrm{N}(2)$ & $0.28(14)$ & $\mathrm{C}(1)-\mathrm{C}(2)-\mathrm{C}(3)-\mathrm{C}(4)$ & $-177.03(13)$ \\
\hline $\mathrm{C}(8)-\mathrm{N}(3)-\mathrm{C}(4)-\mathrm{C}(5)$ & $-1.25(18)$ & $\mathrm{C}(8)-\mathrm{N}(3)-\mathrm{C}(4)-\mathrm{C}(3)$ & $-178.99(11)$ \\
\hline $\mathrm{N}(2)-\mathrm{C}(3)-\mathrm{C}(4)-\mathrm{N}(3)$ & $6.54(17)$ & $\mathrm{C}(2)-\mathrm{C}(3)-\mathrm{C}(4)-\mathrm{N}(3)$ & $-176.46(13)$ \\
\hline $\mathrm{N}(2)-\mathrm{C}(3)-\mathrm{C}(4)-\mathrm{C}(5)$ & $-171.24(12)$ & $\mathrm{C}(2)-\mathrm{C}(3)-\mathrm{C}(4)-\mathrm{C}(5)$ & $5.8(2)$ \\
\hline $\mathrm{N}(3)-\mathrm{C}(4)-\mathrm{C}(5)-\mathrm{C}(6)$ & $0.94(19)$ & $\mathrm{C}(3)-\mathrm{C}(4)-\mathrm{C}(5)-\mathrm{C}(6)$ & $178.54(12)$ \\
\hline $\mathrm{C}(4)-\mathrm{C}(5)-\mathrm{C}(6)-\mathrm{C}(7)$ & $0.5(2)$ & $C(5)-C(6)-C(7)-C(8)$ & $-1.4(2)$ \\
\hline $\mathrm{C}(4)-\mathrm{N}(3)-\mathrm{C}(8)-\mathrm{C}(7)$ & $0.19(18)$ & $\mathrm{C}(4)-\mathrm{N}(3)-\mathrm{C}(8)-\mathrm{C}(9)$ & $178.44(11)$ \\
\hline $\mathrm{C}(6)-\mathrm{C}(7)-\mathrm{C}(8)-\mathrm{N}(3)$ & $1.1(2)$ & $\mathrm{C}(6)-\mathrm{C}(7)-\mathrm{C}(8)-\mathrm{C}(9)$ & $-177.04(12)$ \\
\hline $\mathrm{N}(5)-\mathrm{N}(4)-\mathrm{C}(9)-\mathrm{N}(6)$ & 8.66(19) & $\mathrm{N}(5)-\mathrm{N}(4)-\mathrm{C}(9)-\mathrm{C}(8)$ & $-172.34(11)$ \\
\hline $\mathrm{C}(11)-\mathrm{N}(6)-\mathrm{C}(9)-\mathrm{N}(4)$ & $-3.44(19)$ & $\mathrm{C}(11)-\mathrm{N}(6)-\mathrm{C}(9)-\mathrm{C}(8)$ & $177.56(11)$ \\
\hline $\mathrm{N}(3)-\mathrm{C}(8)-\mathrm{C}(9)-\mathrm{N}(4)$ & $-14.26(17)$ & $\mathrm{C}(7)-\mathrm{C}(8)-\mathrm{C}(9)-\mathrm{N}(4)$ & $164.05(12)$ \\
\hline $\mathrm{N}(3)-\mathrm{C}(8)-\mathrm{C}(9)-\mathrm{N}(6)$ & 164.81(11) & $\mathrm{C}(7)-\mathrm{C}(8)-\mathrm{C}(9)-\mathrm{N}(6)$ & $-16.87(17)$ \\
\hline $\mathrm{N}(4)-\mathrm{N}(5)-\mathrm{C}(10)-\mathrm{C}(11)$ & $-4.53(18)$ & $\mathrm{N}(4)-\mathrm{N}(5)-\mathrm{C}(10)-\mathrm{C}(19)$ & $173.42(11)$ \\
\hline $\mathrm{C}(9)-\mathrm{N}(6)-\mathrm{C}(11)-\mathrm{C}(10)$ & $-5.73(17)$ & $\mathrm{C}(9)-\mathrm{N}(6)-\mathrm{C}(11)-\mathrm{C}(25)$ & $172.94(11)$ \\
\hline $\mathrm{N}(5)-\mathrm{C}(10)-\mathrm{C}(11)-\mathrm{N}(6)$ & $9.78(18)$ & $\mathrm{C}(19)-\mathrm{C}(10)-\mathrm{C}(11)-\mathrm{N}(6)$ & $-167.96(12)$ \\
\hline $\mathrm{N}(5)-\mathrm{C}(10)-\mathrm{C}(11)-\mathrm{C}(25)$ & $-168.81(12)$ & $\mathrm{C}(19)-\mathrm{C}(10)-\mathrm{C}(11)-\mathrm{C}(25)$ & $13.46(19)$ \\
\hline $\mathrm{N}(1)-\mathrm{C}(1)-\mathrm{C}(12)-\mathrm{C}(13)$ & 170.97(12) & $\mathrm{C}(2)-\mathrm{C}(1)-\mathrm{C}(12)-\mathrm{C}(13)$ & $-8.1(2)$ \\
\hline $\mathrm{N}(1)-\mathrm{C}(1)-\mathrm{C}(12)-\mathrm{C}(17)$ & $-7.89(18)$ & $\mathrm{C}(2)-\mathrm{C}(1)-\mathrm{C}(12)-\mathrm{C}(17)$ & $173.02(13)$ \\
\hline $\mathrm{C}(17)-\mathrm{C}(12)-\mathrm{C}(13)-\mathrm{C}(14)$ & $-0.6(2)$ & $\mathrm{C}(1)-\mathrm{C}(12)-\mathrm{C}(13)-\mathrm{C}(14)$ & $-179.49(12)$ \\
\hline C(12)-C(13)-C(14)-C(15) & $1.0(2)$ & $C(13)-C(14)-C(15)-C(16)$ & $-0.5(2)$ \\
\hline $\mathrm{C}(13)-\mathrm{C}(14)-\mathrm{C}(15)-\mathrm{C}(18)$ & $178.48(13)$ & $\mathrm{C}(14)-\mathrm{C}(15)-\mathrm{C}(16)-\mathrm{C}(17)$ & $-0.3(2)$ \\
\hline $\mathrm{C}(18)-\mathrm{C}(15)-\mathrm{C}(16)-\mathrm{C}(17)$ & $-179.31(12)$ & $\mathrm{C}(15)-\mathrm{C}(16)-\mathrm{C}(17)-\mathrm{C}(12)$ & $0.7(2)$ \\
\hline $\mathrm{C}(13)-\mathrm{C}(12)-\mathrm{C}(17)-\mathrm{C}(16)$ & $-0.23(19)$ & $\mathrm{C}(1)-\mathrm{C}(12)-\mathrm{C}(17)-\mathrm{C}(16)$ & $178.66(12)$ \\
\hline $\mathrm{N}(5)-\mathrm{C}(10)-\mathrm{C}(19)-\mathrm{C}(24)$ & $-139.25(13)$ & $\mathrm{C}(11)-\mathrm{C}(10)-\mathrm{C}(19)-\mathrm{C}(24)$ & 38.58(19) \\
\hline $\mathrm{N}(5)-\mathrm{C}(10)-\mathrm{C}(19)-\mathrm{C}(20)$ & $35.14(17)$ & $\mathrm{C}(11)-\mathrm{C}(10)-\mathrm{C}(19)-\mathrm{C}(20)$ & $-147.03(13)$ \\
\hline $\mathrm{C}(24)-\mathrm{C}(19)-\mathrm{C}(20)-\mathrm{C}(21)$ & $-0.1(2)$ & $\mathrm{C}(10)-\mathrm{C}(19)-\mathrm{C}(20)-\mathrm{C}(21)$ & $-174.63(12)$ \\
\hline C(19)-C(20)-C(21)-C(22) & $0.2(2)$ & $\mathrm{C}(20)-\mathrm{C}(21)-\mathrm{C}(22)-\mathrm{C}(23)$ & $0.0(2)$ \\
\hline C(21)-C(22)-C(23)-C(24) & $-0.4(2)$ & $C(22)-C(23)-C(24)-C(19)$ & $0.4(2)$ \\
\hline C(20)-C(19)-C(24)-C(23) & $-0.19(19)$ & $\mathrm{C}(10)-\mathrm{C}(19)-\mathrm{C}(24)-\mathrm{C}(23)$ & $174.23(12)$ \\
\hline $\mathrm{N}(6)-\mathrm{C}(11)-\mathrm{C}(25)-\mathrm{C}(26)$ & $55.60(17)$ & $\mathrm{C}(10)-\mathrm{C}(11)-\mathrm{C}(25)-\mathrm{C}(26)$ & $-125.79(14)$ \\
\hline $\mathrm{N}(6)-\mathrm{C}(11)-\mathrm{C}(25)-\mathrm{C}(30)$ & $-122.94(14)$ & $\mathrm{C}(10)-\mathrm{C}(11)-\mathrm{C}(25)-\mathrm{C}(30)$ & $55.67(18)$ \\
\hline$C(30)-C(25)-C(26)-C(27)$ & $-1.6(2)$ & $C(11)-C(25)-C(26)-C(27)$ & $179.90(12)$ \\
\hline $\mathrm{C}(25)-\mathrm{C}(26)-\mathrm{C}(27)-\mathrm{C}(28)$ & $2.7(2)$ & $\mathrm{C}(26)-\mathrm{C}(27)-\mathrm{C}(28)-\mathrm{C}(29)$ & $-1.2(2)$ \\
\hline $\mathrm{C}(27)-\mathrm{C}(28)-\mathrm{C}(29)-\mathrm{C}(30)$ & $-1.4(2)$ & $\mathrm{C}(28)-\mathrm{C}(29)-\mathrm{C}(30)-\mathrm{C}(25)$ & $2.5(2)$ \\
\hline $\mathrm{C}(26)-\mathrm{C}(25)-\mathrm{C}(30)-\mathrm{C}(29)$ & $-1.0(2)$ & $\mathrm{C}(11)-\mathrm{C}(25)-\mathrm{C}(30)-\mathrm{C}(29)$ & $177.50(13)$ \\
\hline $\mathrm{C}(6 \mathrm{~S})-\mathrm{O}(2 \mathrm{~S})-\mathrm{C}(3 \mathrm{~S})-\mathrm{C}(4 \mathrm{~S})$ & $35.2(2)$ & $\mathrm{O}(2 \mathrm{~S})-\mathrm{C}(3 \mathrm{~S})-\mathrm{C}(4 \mathrm{~S})-\mathrm{C}(5 \mathrm{~S})$ & $-22.8(3)$ \\
\hline $\mathrm{C}(3 \mathrm{~S})-\mathrm{C}(4 \mathrm{~S})-\mathrm{C}(5 \mathrm{~S})-\mathrm{C}(6 \mathrm{~S})$ & $2.3(3)$ & $\mathrm{C}(3 \mathrm{~S})-\mathrm{O}(2 \mathrm{~S})-\mathrm{C}(6 \mathrm{~S})-\mathrm{C}(5 \mathrm{~S})$ & $-33.38(19)$ \\
\hline $\mathrm{C}(4 \mathrm{~S})-\mathrm{C}(5 \mathrm{~S})-\mathrm{C}(6 \mathrm{~S})-\mathrm{O}(2 \mathrm{~S})$ & $18.6(2)$ & & \\
\hline
\end{tabular}


Table S7. Hydrogen bonds for VGB-B-279 [ $\AA \AA$ and $\left.{ }^{\circ}\right]$.

D-H...A

$\mathrm{N}(2)-\mathrm{H}(2 \mathrm{~N}) \ldots \mathrm{O}(1 \mathrm{~S})$

$\mathrm{O}(1 \mathrm{~S})-\mathrm{H}(1 \mathrm{OA}) \ldots \mathrm{N}(3)$

$\mathrm{O}(1 \mathrm{~S})-\mathrm{H}(1 \mathrm{OA}) \ldots \mathrm{N}(4)$
$\mathrm{d}(\mathrm{D}-\mathrm{H})$

$0.92(2)$

$0.86(2)$

$0.86(2)$
d(H...A)

$1.92(2)$

$2.65(2)$

$2.08(2)$
d(D...A) $\quad<$ (DHA)

2.8392(15) 170.5(18)

3.1590(15) 118.7(16)

2.9421(15) 172.2(19) 


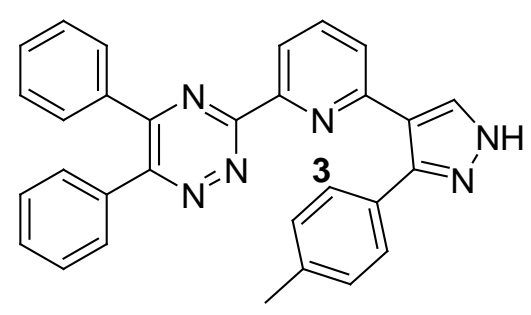

Sample: VGB-B-137(5)

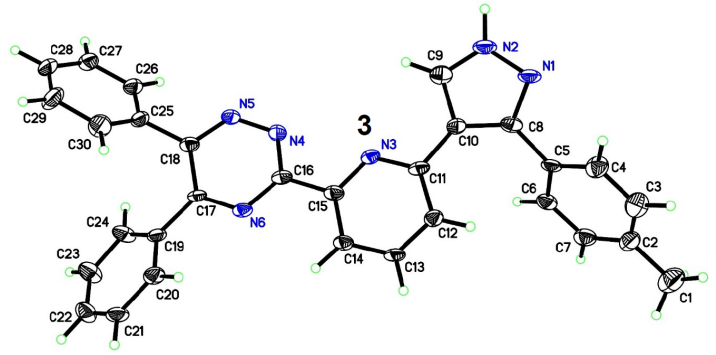

X-ray Structure Report

for

Jesse Carrick

Dept. of Chemistry, Tennessee Tech

jcarrick@tntech.edu

Prof. J. Carrick

Wednesday, April 17, 2019 


\section{$\underline{\text { DISCUSSION }}$}

The compound, 5,6-diphenyl-3-(6-(3-(p-tolyl)-1H-pyrazol-4-yl)pyridin-2-yl)-1,2,4-triazine, crystallizes as colorless tablet-like crystals. There are two molecules of the compound in the unit cell of the primitive, centrosymmetric, triclinic space group P-1.

The structure of the compound is different from that predicted (see Figure). The pyrazole moiety is bonded to the tolyl group and the pyridine at the $\mathrm{C} 1 / \mathrm{C} 2$ positions rather than $\mathrm{C} 1 / \mathrm{C} 3$ positions as proposed. The planes of the core three rings are not co-planar. The plane of the pyrazole rings forms an angle of $20.9(3)^{\circ}$ to the mean plane of the pyridine ring, and the triazine ring forms an angle of $9.7(3)^{\circ}$ also to the plane of the pyridine ring. The atoms forming the triazine ring are also distorted from a planar arrangement, with the largest deviation from the mean plane of N4, N5, N6 C9, C10, C11 being 0.0852(3) ^ for C10 (see listing files for details). The tolyl group is oriented in a periplanar fashion with respect to the pyrazole ring $\left(52.7(2)^{\circ}\right)$ and the phenyl groups are at less of an inclination, with respect to the triazine ring (43.6(3) and 39.1(2) respectively).

The pyrazole nitrogen, N2, forms a bifurcated hydrogen bond with N4 and N5 of a neighboring, inversion-related, molecule (see Table of Hydrogen Bonds for details). This results in a hydrogenbonded dimer of molecules within the lattice.

The structure was modeled as a twin, with an approximately 0.82:0.18 major:minor domain ratio. The twin relationship ship is a $180^{\circ}$ rotation about the reciprocal $c$-axis.

The N1-C1-C12 angle deviates slightly from an expected ideal $120^{\circ}\left(116.3(5)^{\circ}\right.$, see Table of Bond Angles). All other bond distances and angles are as expected.

\section{CRYSTAL SUMMARY}

Crystal data for $\mathrm{C}_{30} \mathrm{H}_{22} \mathrm{~N}_{6} ; \mathrm{M}_{\mathrm{r}}=466.53$; Triclinic; space group P-1; $a=9.117(10) \AA \AA$ 10.951(13) $\AA ; c=13.219(15) \AA ; \alpha=82.747(16)^{\circ} ; \beta=71.248(16)^{\circ} ; \gamma=74.802(14)^{\circ} ; \mathrm{V}=1205(2)$ $\AA^{3} ; \mathrm{Z}=2 ; \mathrm{T}=120(2) \mathrm{K} ; \lambda(\mathrm{Mo}-\mathrm{K} \alpha)=0.71073 \AA ; \mu(\mathrm{Mo}-\mathrm{K} \alpha)=0.079 \mathrm{~mm}^{-1} ; \mathrm{d}_{\mathrm{calc}}=1.286 \mathrm{~g}^{\circ} \mathrm{cm}^{-3}$; 5470 reflections collected; 5470 unique ( $\mathrm{R}_{\text {int }}=$ ?); giving $\mathrm{R}_{1}=0.0949, \mathrm{wR}_{2}=0.2212$ for 3266 data with $[\mathrm{I}>2 \sigma(\mathrm{I})]$ and $\mathrm{R}_{1}=0.1649, \mathrm{wR}_{2}=0.2621$ for all 5470 data. Residual electron density $\left(\mathrm{e}^{-} . \AA^{-3}\right)$ $\max / \min : 0.454 /-0.344$.

An arbitrary sphere of data was collected on a colorless tablet-like crystal, having approximate dimensions of $0.296 \times 0.242 \times 0.087 \mathrm{~mm}$, on a Bruker APEX-II diffractometer using a combination of $\omega$ - and $\varphi$-scans of $0.5^{\circ}$ [1]. Data were corrected for absorption and polarization effects and analyzed for space group determination [2]. The structure was solved by dual-space methods and expanded routinely [3]. The model was refined by full-matrix least-squares analysis of $\mathrm{F}^{2}$ against all reflections [4]. All non-hydrogen atoms were refined with anisotropic atomic displacement parameters. Unless otherwise noted, hydrogen atoms were included in calculated positions. Atomic displacement parameters for the hydrogens were tied to the equivalent isotropic displacement parameter of the atom to which they are bonded $\left(U_{\text {iso }}(\mathrm{H})=1.5 U_{\text {eq }}(\mathrm{C})\right.$ for methyl, $1.2 U_{\text {eq }}(\mathrm{C})$ for all others). 


\section{REFERENCES}

[1] APEX-3. Bruker AXS. Madison, Wisconsin, USA. 2016.

[2] L. Krause, R. Herbst-Irmer, G. M. Sheldrick, \& D. Stalke. J. Appl. Cryst. 2015 48, 3.

[3] G. M. Sheldrick. Acta Cryst., 2015, A71, 3.

[4] G. M. Sheldrick. Acta Cryst., 2015, C71, 3. 
Table S8. Crystal data and structure refinement for VGB-B-137(5).

\begin{tabular}{|c|c|c|}
\hline Identification code & \multicolumn{2}{|l|}{ vgbb137t5 } \\
\hline Empirical formula & \multicolumn{2}{|l|}{$\mathrm{C}_{30} \mathrm{H}_{22} \mathrm{~N}_{6}$} \\
\hline Formula weight & \multicolumn{2}{|l|}{466.53} \\
\hline Temperature & \multicolumn{2}{|l|}{$120(2) \mathrm{K}$} \\
\hline Wavelength & \multicolumn{2}{|l|}{$0.71073 \AA$} \\
\hline Crystal system & \multicolumn{2}{|l|}{ Triclinic } \\
\hline Space group & \multicolumn{2}{|l|}{ P-1 } \\
\hline \multirow[t]{3}{*}{ Unit cell dimensions } & $a=9.117(10) \AA$ & $\alpha=82.747(16)^{\circ}$ \\
\hline & $b=10.951(13) \AA$ & $\beta=71.248(16)^{\circ}$ \\
\hline & $c=13.219(15) \AA$ & $\gamma=74.802(14)^{\circ}$ \\
\hline Volume & \multicolumn{2}{|l|}{$1205(2) \AA^{3}$} \\
\hline $\mathrm{Z}$ & \multicolumn{2}{|l|}{2} \\
\hline Density (calculated) & \multicolumn{2}{|l|}{1.286 g.cm $\mathrm{cm}^{-3}$} \\
\hline Absorption coefficient $(\mu)$ & \multicolumn{2}{|l|}{$0.079 \mathrm{~mm}^{-1}$} \\
\hline $\mathrm{F}(000)$ & \multicolumn{2}{|l|}{488} \\
\hline Crystal color, habit & \multicolumn{2}{|l|}{ colorless, tablet } \\
\hline Crystal size & \multicolumn{2}{|c|}{$0.296 \times 0.242 \times 0.087 \mathrm{~mm}^{3}$} \\
\hline$\theta$ range for data collection & \multicolumn{2}{|l|}{1.629 to $27.367^{\circ}$} \\
\hline Index ranges & \multicolumn{2}{|c|}{$-10 \leq \mathrm{h} \leq 11,-13 \leq \mathrm{k} \leq 14,0 \leq 1 \leq 17$} \\
\hline Reflections collected & \multicolumn{2}{|l|}{5470} \\
\hline Independent reflections & \multicolumn{2}{|l|}{$5470\left[\mathrm{R}_{\text {int }}=\right.$ ?] } \\
\hline Completeness to $\theta=25.242^{\circ}$ & \multicolumn{2}{|l|}{$99.3 \%$} \\
\hline Absorption correction & \multicolumn{2}{|c|}{ Semi-empirical from equivalents } \\
\hline Max. and min. transmission & \multicolumn{2}{|l|}{0.7455 and 0.5974} \\
\hline Refinement method & \multicolumn{2}{|c|}{ Full-matrix least-squares on $\mathrm{F}^{2}$} \\
\hline Data / restraints / parameters & \multicolumn{2}{|l|}{5470 / 0 / 331} \\
\hline Goodness-of-fit on $\mathrm{F}^{2}$ & \multicolumn{2}{|l|}{1.027} \\
\hline Final $R$ indices $[\mathrm{I}>2 \sigma(\mathrm{I})]$ & \multicolumn{2}{|c|}{$\mathrm{R}_{1}=0.0949, \mathrm{wR}_{2}=0.2212$} \\
\hline $\mathrm{R}$ indices (all data) & \multicolumn{2}{|c|}{$\mathrm{R}_{1}=0.1649, \mathrm{wR}_{2}=0.2621$} \\
\hline Extinction coefficient & \multicolumn{2}{|l|}{$\mathrm{n} / \mathrm{a}$} \\
\hline Largest diff. peak and hole & \multicolumn{2}{|c|}{0.454 and $-0.344 \mathrm{e}^{-} . \AA^{-3}$} \\
\hline
\end{tabular}


Table S9. Atomic coordinates and equivalent isotropic displacement parameters $\left(\AA^{2}\right)$ for VGB-B-137(5).

$\mathrm{U}(\mathrm{eq})$ is defined as one third of the trace of the orthogonalized $\mathrm{U}_{\mathrm{ij}}$ tensor.

\begin{tabular}{|c|c|c|c|c|}
\hline & $\mathrm{x}$ & $\mathrm{y}$ & $\mathrm{z}$ & $\mathrm{U}(\mathrm{eq})$ \\
\hline $\mathrm{N}(1)$ & $1.2294(6)$ & $0.1630(4)$ & $0.6887(4)$ & $0.036(1)$ \\
\hline $\mathrm{N}(2)$ & $1.2258(6)$ & $0.2568(4)$ & $0.6103(4)$ & $0.036(1)$ \\
\hline $\mathrm{N}(3)$ & $0.8211(5)$ & $0.2494(4)$ & $0.5335(3)$ & $0.026(1)$ \\
\hline $\mathrm{N}(4)$ & $0.6756(5)$ & $0.4518(4)$ & $0.4281(3)$ & $0.027(1)$ \\
\hline $\mathrm{N}(5)$ & $0.6295(5)$ & $0.5413(4)$ & $0.3580(3)$ & $0.029(1)$ \\
\hline $\mathrm{N}(6)$ & $0.5320(5)$ & $0.3206(4)$ & $0.3857(3)$ & $0.026(1)$ \\
\hline $\mathrm{C}(1)$ & $1.1054(6)$ & $0.1131(5)$ & $0.6933(4)$ & $0.029(1)$ \\
\hline $\mathrm{C}(2)$ & $1.0240(6)$ & $0.1763(4)$ & $0.6200(4)$ & $0.028(1)$ \\
\hline C(3) & $1.1066(7)$ & $0.2699(5)$ & $0.5682(4)$ & $0.031(1)$ \\
\hline C(4) & $0.8916(6)$ & $0.1536(4)$ & $0.5887(4)$ & $0.024(1)$ \\
\hline$C(5)$ & $0.8484(6)$ & $0.0355(5)$ & $0.6096(4)$ & $0.028(1)$ \\
\hline C(6) & $0.7290(6)$ & $0.0196(5)$ & $0.5717(4)$ & $0.028(1)$ \\
\hline C(7) & $0.6560(6)$ & $0.1177(4)$ & $0.5130(4)$ & $0.026(1)$ \\
\hline $\mathrm{C}(8)$ & $0.7063(6)$ & $0.2317(5)$ & $0.4952(4)$ & $0.025(1)$ \\
\hline C(9) & $0.6366(6)$ & $0.3391(5)$ & $0.4324(4)$ & $0.026(1)$ \\
\hline$C(10)$ & $0.5429(6)$ & $0.5239(4)$ & $0.2982(4)$ & $0.027(1)$ \\
\hline $\mathrm{C}(11)$ & $0.4764(6)$ & $0.4145(4)$ & $0.3237(4)$ & $0.024(1)$ \\
\hline $\mathrm{C}(12)$ & $1.0725(6)$ & $0.0098(5)$ & $0.7776(4)$ & $0.027(1)$ \\
\hline C(13) & $0.9218(7)$ & $0.0196(5)$ & $0.8532(4)$ & $0.033(1)$ \\
\hline $\mathrm{C}(14)$ & $0.8954(7)$ & $-0.0743(5)$ & $0.9343(4)$ & $0.035(1)$ \\
\hline$C(15)$ & $1.0136(8)$ & $-0.1794(5)$ & $0.9434(4)$ & $0.039(1)$ \\
\hline$C(16)$ & $1.1653(8)$ & $-0.1897(6)$ & $0.8666(5)$ & $0.046(2)$ \\
\hline $\mathrm{C}(17)$ & $1.1926(8)$ & $-0.0960(5)$ & $0.7853(5)$ & $0.040(1)$ \\
\hline C(18) & $0.9850(10)$ & $-0.2832(6)$ & $1.0313(5)$ & $0.055(2)$ \\
\hline C(19) & $0.5255(7)$ & $0.6198(5)$ & $0.2103(4)$ & $0.029(1)$ \\
\hline$C(20)$ & $0.5439(9)$ & $0.5811(6)$ & $0.1091(5)$ & $0.053(2)$ \\
\hline $\mathrm{C}(21)$ & $0.5415(12)$ & $0.6670(7)$ & $0.0240(5)$ & $0.072(3)$ \\
\hline $\mathrm{C}(22)$ & $0.5152(9)$ & $0.7975(6)$ & $0.0388(5)$ & $0.051(2)$ \\
\hline C(23) & $0.4994(7)$ & $0.8367(5)$ & $0.1380(5)$ & $0.039(1)$ \\
\hline$C(24)$ & $0.5084(6)$ & $0.7487(5)$ & $0.2237(4)$ & $0.029(1)$ \\
\hline$C(25)$ & $0.3425(6)$ & $0.4008(5)$ & $0.2874(4)$ & $0.029(1)$ \\
\hline$C(26)$ & $0.3396(7)$ & $0.2799(5)$ & $0.2635(4)$ & $0.033(1)$ \\
\hline $\mathrm{C}(27)$ & $0.2100(7)$ & $0.2641(5)$ & $0.2352(5)$ & $0.037(1)$ \\
\hline C(28) & $0.0837(8)$ & $0.3670(6)$ & $0.2317(4)$ & $0.040(1)$ \\
\hline C(29) & $0.0843(8)$ & $0.4866(6)$ & $0.2568(5)$ & $0.043(2)$ \\
\hline C(30) & $0.2146(7)$ & $0.5040(5)$ & $0.2845(5)$ & $0.036(1)$ \\
\hline $\mathrm{H}(2 \mathrm{~N})$ & $1.295(8)$ & $0.313(6)$ & $0.599(5)$ & $0.043(17)$ \\
\hline $\mathrm{H}(3)$ & 1.08259 & 0.33043 & 0.51419 & 0.038 \\
\hline $\mathrm{H}(5)$ & 0.89982 & -0.03139 & 0.64867 & 0.034 \\
\hline
\end{tabular}




$\begin{array}{lrrrr}\mathrm{H}(6) & 0.69707 & -0.05837 & 0.58587 & 0.034 \\ \mathrm{H}(7) & 0.57519 & 0.10793 & 0.48603 & 0.031 \\ \mathrm{H}(13) & 0.83721 & 0.09050 & 0.84924 & 0.040 \\ \mathrm{H}(14) & 0.79242 & -0.06540 & 0.98486 & 0.042 \\ \mathrm{H}(16) & 1.24954 & -0.26100 & 0.87031 & 0.055 \\ \mathrm{H}(17) & 1.29531 & -0.10483 & 0.73434 & 0.047 \\ \mathrm{H}(18 \mathrm{~A}) & 1.08737 & -0.33984 & 1.03256 & 0.082 \\ \mathrm{H}(18 \mathrm{~B}) & 0.91801 & -0.33174 & 1.01703 & 0.082 \\ \mathrm{H}(18 \mathrm{C}) & 0.93120 & -0.24450 & 1.10064 & 0.082 \\ \mathrm{H}(20) & 0.55834 & 0.49386 & 0.09906 & 0.064 \\ \mathrm{H}(21) & 0.55751 & 0.63871 & -0.04469 & 0.086 \\ \mathrm{H}(22) & 0.50834 & 0.85775 & -0.01891 & 0.061 \\ \mathrm{H}(23) & 0.48224 & 0.92432 & 0.14809 & 0.047 \\ \mathrm{H}(24) & 0.50306 & 0.77587 & 0.29056 & 0.035 \\ \mathrm{H}(26) & 0.42495 & 0.20924 & 0.26656 & 0.039 \\ \mathrm{H}(27) & 0.20852 & 0.18269 & 0.21834 & 0.044 \\ \mathrm{H}(28) & -0.00322 & 0.35558 & 0.21211 & 0.048 \\ \mathrm{H}(29) & -0.00281 & 0.55631 & 0.25537 & 0.052 \\ \mathrm{H}(30) & 0.21558 & 0.58569 & 0.30107 & 0.044\end{array}$


Table S10. Anisotropic displacement parameters $\left(\AA^{2}\right)$ for VGB-B-137(5).

The anisotropic displacement factor exponent takes the form:

$-2 \pi^{2}\left[h^{2} a^{* 2} U_{11}+\ldots+2 h k a * b * U_{12}\right]$

\begin{tabular}{|c|c|c|c|c|c|c|}
\hline & $\mathrm{U}_{11}$ & $\mathrm{U}_{22}$ & $\mathrm{U}_{33}$ & $\mathrm{U}_{23}$ & $\mathrm{U}_{13}$ & $\mathrm{U}_{12}$ \\
\hline $\mathrm{N}(1)$ & $0.037(3)$ & $0.029(2)$ & $0.043(3)$ & $-0.004(2)$ & $-0.009(2)$ & $-0.013(2)$ \\
\hline$N(2)$ & $0.035(3)$ & $0.031(2)$ & $0.042(3)$ & $-0.003(2)$ & $-0.007(2)$ & $-0.016(2)$ \\
\hline$N(3)$ & $0.032(2)$ & $0.023(2)$ & $0.021(2)$ & $-0.0041(17)$ & $-0.0020(18)$ & $-0.0108(1$ \\
\hline$N(4)$ & $0.035(3)$ & $0.0196(19)$ & $0.029(2)$ & $0.0035(17)$ & $-0.0102(19)$ & $-0.0140(18$ \\
\hline$N(5)$ & $0.034(3)$ & $0.026(2)$ & $0.029(2)$ & $-0.0035(18)$ & $-0.0046(19)$ & $-0.0164(19)$ \\
\hline$N(6)$ & $0.031(2)$ & $0.023(2)$ & $0.023(2)$ & $-0.0092(17)$ & $0.0007(18)$ & $-0.0106(18$ \\
\hline$C(1)$ & $0.031(3)$ & $0.024(2)$ & $0.034(3)$ & $-0.008(2)$ & $-0.005(2)$ & $-0.013(2)$ \\
\hline$C(2)$ & $0.030(3)$ & $0.021(2)$ & $0.033(3)$ & $-0.006(2)$ & $-0.002(2)$ & $-0.012(2)$ \\
\hline (3) & $0.035(3)$ & $0.025(3)$ & $0.033(3)$ & $-0.008(2)$ & $-0.005(2)$ & $-0.009(2)$ \\
\hline (4) & .025(3) & $0.024(2)$ & $0.022(2)$ & $-0.0073(19)$ & $0.000(2)$ & $-0.013(2)$ \\
\hline (5) & .037(3) & $.020(2)$ & $0.024(2)$ & $-0.006(2)$ & $-0.002(2)$ & $-0.008(2)$ \\
\hline C(6) & 033(3) & 0.02 & 0.02 & -0.00 & -0.00 & $-0.016(2)$ \\
\hline C(7) & $0.036(3)$ & 0.023 & $0.024(2)$ & -0.00 & -0.00 & -0.0 \\
\hline C(8) & $.030(3)$ & $0.024(2)$ & $0.022(2)$ & $-0.0052(19)$ & $-0.002(2)$ & $-0.012(2)$ \\
\hline C(9) & .033(3) & 0.02 & $0.021(2)$ & -0.00 & $0.004(2)$ & $-0.019(2)$ \\
\hline (10) & 031(3) & 0.02 & $0.026(2)$ & -0.00 & -0.00 & -0.0 \\
\hline C(11) & $0.028(3)$ & $0.018(2)$ & $0.025(2)$ & -0.00 & $0.001(2)$ & -0.0 \\
\hline$C(12)$ & $0.036(3)$ & $0.026(2)$ & $0.026(3)$ & $-0.002(2)$ & $-0.013(2)$ & $-0.014(2)$ \\
\hline C(13) & $0.030(3)$ & $0.032(3)$ & $0.040(3)$ & -0.0 & -0.0 & -0.0 \\
\hline C(14) & 03 & .03 & 0.03 & -0.00 & -0.00 & $-0.019(3)$ \\
\hline C(15) & 0.05 & 0.03 & $0.030(3)$ & 0.00 & -0.01 & $-0.025(3)$ \\
\hline$C(16)$ & $0.050(4)$ & $0.037(3)$ & $0.043(3)$ & $-0.006(3)$ & $-0.007(3)$ & $-0.007(3)$ \\
\hline C(17) & $0.045(4)$ & $0.039(3)$ & $0.034(3)$ & -0.00 & $-0.006(3)$ & $-0.015(3)$ \\
\hline C(18) & 007 & 4) & 0.02 & -0.00 & -0.01 & -0.0 \\
\hline C(19) & 0.03 & 0.02 & $0.029(3)$ & 0.00 & $-0.007(2)$ & $-0.022(2)$ \\
\hline C(20) & $0.095(6)$ & $0.042(3)$ & $0.040(3)$ & 0.00 & $-0.025(4)$ & $-0.041(4)$ \\
\hline$C(21)$ & $0.147(8)$ & $0.061(4)$ & $0.035(4)$ & $0.015(3)$ & $-0.031(4)$ & $-0.075(5)$ \\
\hline$C(22)$ & $0.082(5)$ & 0046 (2) & $0.037(3)$ & $0.013(3)$ & $-0.019(3)$ & $-0.043(4)$ \\
\hline C(23) & תכ0 ח & (20 & 0.04 & -0.00 & -0.00 & -0.0 \\
\hline$C(24)$ & 0.03 & 0.02 & $0.027(3)$ & -0.0 & -0.0 & -0.0 \\
\hline$C(25)$ & $0.035(3)$ & $0.025(3)$ & $0.029(3)$ & $-0.004(2)$ & $-0.003(2)$ & $-0.018(2)$ \\
\hline C(26) & $0.047(3)$ & $0.028(3)$ & $0.028(3)$ & $-0.003(2)$ & $-0.007(2)$ & $-0.021(3)$ \\
\hline C(27) & $047(4)$ & 0.03 & $0.037(3)$ & $-0.006(2)$ & $-0.007(3)$ & $-0.025(3)$ \\
\hline C(28 & & & & $-0.008(3)$ & $-0.010(3)$ & $-0.024(3)$ \\
\hline C(29) & $0.045(4)$ & $0.039(3)$ & $0.052(4)$ & $-0.009(3)$ & $-0.019(3)$ & $-0.008(3)$ \\
\hline C(30) & $0.039(3)$ & $0.034(3)$ & $0.044(3)$ & $-0.007(2)$ & $-0.016(3)$ & $-0.014(3)$ \\
\hline
\end{tabular}


Table S11. Bond lengths [Å] for VGB-B-137(5).

$\begin{array}{llll}\text { atom-atom } & \text { distance } & \text { atom-atom } & \text { distance } \\ \mathrm{N}(1)-\mathrm{C}(1) & & & \\ \mathrm{N}(2)-\mathrm{C}(3) & 1.360(7) & \mathrm{N}(1)-\mathrm{N}(2) & 1.366(7) \\ \mathrm{N}(3)-\mathrm{C}(4) & 1.341(8) & \mathrm{N}(2)-\mathrm{H}(2 \mathrm{~N}) & 0.96(6) \\ \mathrm{N}(4)-\mathrm{N}(5) & 1.342(7) & \mathrm{N}(3)-\mathrm{C}(8) & 1.365(7) \\ \mathrm{N}(5)-\mathrm{C}(10) & 1.342(6) & \mathrm{N}(4)-\mathrm{C}(9) & 1.361(6) \\ \mathrm{N}(6)-\mathrm{C}(9) & 1.343(7) & \mathrm{N}(6)-\mathrm{C}(11) & 1.329(7) \\ \mathrm{C}(1)-\mathrm{C}(12) & 1.358(7) & \mathrm{C}(1)-\mathrm{C}(2) & 1.412(8) \\ \mathrm{C}(2)-\mathrm{C}(4) & 1.503(7) & \mathrm{C}(2)-\mathrm{C}(3) & 1.408(7) \\ \mathrm{C}(4)-\mathrm{C}(5) & 1.481(7) & \mathrm{C}(3)-\mathrm{H}(3) & 0.9500 \\ \mathrm{C}(5)-\mathrm{H}(5) & 1.418(7) & \mathrm{C}(5)-\mathrm{C}(6) & 1.392(8) \\ \mathrm{C}(6)-\mathrm{H}(6) & 0.9500 & \mathrm{C}(6)-\mathrm{C}(7) & 1.395(7) \\ \mathrm{C}(7)-\mathrm{H}(7) & 0.9500 & \mathrm{C}(7)-\mathrm{C}(8) & 1.407(7) \\ \mathrm{C}(10)-\mathrm{C}(11) & 0.9500 & \mathrm{C}(8)-\mathrm{C}(9) & 1.476(7) \\ \mathrm{C}(11)-\mathrm{C}(25) & 1.437(6) & \mathrm{C}(10)-\mathrm{C}(19) & 1.483(7) \\ \mathrm{C}(12)-\mathrm{C}(13) & 1.494(8) & \mathrm{C}(12)-\mathrm{C}(17) & 1.388(8) \\ \mathrm{C}(13)-\mathrm{H}(13) & 1.402(8) & \mathrm{C}(13)-\mathrm{C}(14) & 1.396(8) \\ \mathrm{C}(14)-\mathrm{H}(14) & 0.9500 & \mathrm{C}(14)-\mathrm{C}(15) & 1.378(9) \\ \mathrm{C}(15)-\mathrm{C}(18) & 0.9500 & \mathrm{C}(15)-\mathrm{C}(16) & 1.415(9) \\ \mathrm{C}(16)-\mathrm{H}(16) & 1.528(8) & \mathrm{C}(16)-\mathrm{C}(17) & 1.398(9) \\ \mathrm{C}(18)-\mathrm{H}(18 \mathrm{~A}) & 0.9500 & \mathrm{C}(17)-\mathrm{H}(17) & 0.9500 \\ \mathrm{C}(18)-\mathrm{H}(18 \mathrm{C}) & 0.9800 & \mathrm{C}(18)-\mathrm{H}(18 \mathrm{~B}) & 0.9800 \\ \mathrm{C}(19)-\mathrm{C}(24) & 0.9800 & \mathrm{C}(19)-\mathrm{C}(20) & 1.400(8) \\ \mathrm{C}(20)-\mathrm{H}(20) & 1.406(7) & \mathrm{C}(20)-\mathrm{C}(21) & 1.375(9) \\ \mathrm{C}(21)-\mathrm{H}(21) & 0.9500 & \mathrm{C}(21)-\mathrm{C}(22) & 1.414(9) \\ \mathrm{C}(22)-\mathrm{H}(22) & 0.9500 & \mathrm{C}(22)-\mathrm{C}(23) & 1.383(9) \\ \mathrm{C}(23)-\mathrm{H}(23) & 0.9500 & \mathrm{C}(23)-\mathrm{C}(24) & 1.402(8) \\ \mathrm{C}(25)-\mathrm{C}(30) & 0.9500 & \mathrm{C}(24)-\mathrm{H}(24) & 0.9500 \\ \mathrm{C}(26)-\mathrm{C}(27) & 1.402(8) & \mathrm{C}(25)-\mathrm{C}(26) & 1.409(7) \\ \mathrm{C}(27)-\mathrm{C}(28) & 1.405(8) & \mathrm{C}(26)-\mathrm{H}(26) & 0.9500 \\ \mathrm{C}(28)-\mathrm{C}(29) & 1.392(9) & \mathrm{C}(27)-\mathrm{H}(27) & 0.9500 \\ \mathrm{C}(29)-\mathrm{C}(30) & 1.393(8) & \mathrm{C}(28)-\mathrm{H}(28) & 0.9500 \\ \mathrm{C}(30)-\mathrm{H}(30) & 1.414(8) & \mathrm{C}(29)-\mathrm{H}(29) & 0.9500 \\ & 0.9500 & & \end{array}$

Symmetry transformations used to generate equivalent atoms: 
Table S12. Bond angles $\left[^{\circ}\right]$ for VGB-B-137(5).

\begin{tabular}{|c|c|c|c|}
\hline atom-atom-atom & angle & atom-atom-atom & angle \\
\hline $\mathrm{C}(1)-\mathrm{N}(1)-\mathrm{N}(2)$ & $103.4(4)$ & $\mathrm{C}(3)-\mathrm{N}(2)-\mathrm{N}(1)$ & $113.9(5)$ \\
\hline $\mathrm{C}(3)-\mathrm{N}(2)-\mathrm{H}(2 \mathrm{~N})$ & $127(4)$ & $\mathrm{N}(1)-\mathrm{N}(2)-\mathrm{H}(2 \mathrm{~N})$ & $119(4)$ \\
\hline C(4)-N(3)-C(8) & $118.8(4)$ & $\mathrm{N}(5)-\mathrm{N}(4)-\mathrm{C}(9)$ & $116.6(4)$ \\
\hline $\mathrm{N}(4)-\mathrm{N}(5)-\mathrm{C}(10)$ & $122.3(4)$ & $\mathrm{C}(11)-\mathrm{N}(6)-\mathrm{C}(9)$ & $117.9(4)$ \\
\hline $\mathrm{N}(1)-\mathrm{C}(1)-\mathrm{C}(2)$ & $111.8(4)$ & $\mathrm{N}(1)-\mathrm{C}(1)-\mathrm{C}(12)$ & $116.3(5)$ \\
\hline $\mathrm{C}(2)-\mathrm{C}(1)-\mathrm{C}(12)$ & $131.7(5)$ & $\mathrm{C}(3)-\mathrm{C}(2)-\mathrm{C}(1)$ & $104.4(4)$ \\
\hline$C(3)-C(2)-C(4)$ & $122.5(5)$ & $C(1)-C(2)-C(4)$ & $132.9(4)$ \\
\hline $\mathrm{N}(2)-\mathrm{C}(3)-\mathrm{C}(2)$ & $106.5(5)$ & $\mathrm{N}(2)-\mathrm{C}(3)-\mathrm{H}(3)$ & 126.8 \\
\hline $\mathrm{C}(2)-\mathrm{C}(3)-\mathrm{H}(3)$ & 126.8 & $\mathrm{~N}(3)-\mathrm{C}(4)-\mathrm{C}(5)$ & $121.9(4)$ \\
\hline $\mathrm{N}(3)-\mathrm{C}(4)-\mathrm{C}(2)$ & $115.7(4)$ & $\mathrm{C}(5)-\mathrm{C}(4)-\mathrm{C}(2)$ & $122.3(5)$ \\
\hline $\mathrm{C}(6)-\mathrm{C}(5)-\mathrm{C}(4)$ & $118.6(5)$ & $\mathrm{C}(6)-\mathrm{C}(5)-\mathrm{H}(5)$ & 120.7 \\
\hline $\mathrm{C}(4)-\mathrm{C}(5)-\mathrm{H}(5)$ & 120.7 & $\mathrm{C}(5)-\mathrm{C}(6)-\mathrm{C}(7)$ & $120.2(5)$ \\
\hline $\mathrm{C}(5)-\mathrm{C}(6)-\mathrm{H}(6)$ & 119.9 & $\mathrm{C}(7)-\mathrm{C}(6)-\mathrm{H}(6)$ & 119.9 \\
\hline $\mathrm{C}(6)-\mathrm{C}(7)-\mathrm{C}(8)$ & $117.7(5)$ & $\mathrm{C}(6)-\mathrm{C}(7)-\mathrm{H}(7)$ & 121.1 \\
\hline $\mathrm{C}(8)-\mathrm{C}(7)-\mathrm{H}(7)$ & 121.1 & $\mathrm{~N}(3)-\mathrm{C}(8)-\mathrm{C}(7)$ & $122.7(5)$ \\
\hline $\mathrm{N}(3)-\mathrm{C}(8)-\mathrm{C}(9)$ & $116.4(4)$ & $\mathrm{C}(7)-\mathrm{C}(8)-\mathrm{C}(9)$ & $120.9(5)$ \\
\hline $\mathrm{N}(6)-\mathrm{C}(9)-\mathrm{N}(4)$ & $123.9(5)$ & N(6)-C(9)-C(8) & $117.7(4)$ \\
\hline N(4)-C(9)-C(8) & $118.4(4)$ & $\mathrm{N}(5)-\mathrm{C}(10)-\mathrm{C}(11)$ & $117.7(5)$ \\
\hline $\mathrm{N}(5)-\mathrm{C}(10)-\mathrm{C}(19)$ & $116.7(4)$ & $\mathrm{C}(11)-\mathrm{C}(10)-\mathrm{C}(19)$ & $125.6(5)$ \\
\hline $\mathrm{N}(6)-\mathrm{C}(11)-\mathrm{C}(10)$ & 119.2(5) & $\mathrm{N}(6)-\mathrm{C}(11)-\mathrm{C}(25)$ & $117.0(4)$ \\
\hline $\mathrm{C}(10)-\mathrm{C}(11)-\mathrm{C}(25)$ & $123.7(5)$ & $\mathrm{C}(17)-\mathrm{C}(12)-\mathrm{C}(13)$ & 118.1(5) \\
\hline $\mathrm{C}(17)-\mathrm{C}(12)-\mathrm{C}(1)$ & $120.8(5)$ & $\mathrm{C}(13)-\mathrm{C}(12)-\mathrm{C}(1)$ & $121.1(5)$ \\
\hline $\mathrm{C}(14)-\mathrm{C}(13)-\mathrm{C}(12)$ & $120.4(5)$ & $\mathrm{C}(14)-\mathrm{C}(13)-\mathrm{H}(13)$ & 119.8 \\
\hline $\mathrm{C}(12)-\mathrm{C}(13)-\mathrm{H}(13)$ & 119.8 & $\mathrm{C}(15)-\mathrm{C}(14)-\mathrm{C}(13)$ & $122.2(5)$ \\
\hline $\mathrm{C}(15)-\mathrm{C}(14)-\mathrm{H}(14)$ & 118.9 & $\mathrm{C}(13)-\mathrm{C}(14)-\mathrm{H}(14)$ & 118.9 \\
\hline $\mathrm{C}(14)-\mathrm{C}(15)-\mathrm{C}(16)$ & $117.3(5)$ & $\mathrm{C}(14)-\mathrm{C}(15)-\mathrm{C}(18)$ & $122.5(6)$ \\
\hline $\mathrm{C}(16)-\mathrm{C}(15)-\mathrm{C}(18)$ & $120.2(6)$ & $\mathrm{C}(17)-\mathrm{C}(16)-\mathrm{C}(15)$ & $120.7(6)$ \\
\hline $\mathrm{C}(17)-\mathrm{C}(16)-\mathrm{H}(16)$ & 119.6 & $\mathrm{C}(15)-\mathrm{C}(16)-\mathrm{H}(16)$ & 119.6 \\
\hline $\mathrm{C}(12)-\mathrm{C}(17)-\mathrm{C}(16)$ & $121.2(6)$ & $\mathrm{C}(12)-\mathrm{C}(17)-\mathrm{H}(17)$ & 119.4 \\
\hline $\mathrm{C}(16)-\mathrm{C}(17)-\mathrm{H}(17)$ & 119.4 & $\mathrm{C}(15)-\mathrm{C}(18)-\mathrm{H}(18 \mathrm{~A})$ & 109.5 \\
\hline $\mathrm{C}(15)-\mathrm{C}(18)-\mathrm{H}(18 \mathrm{~B})$ & 109.5 & $\mathrm{H}(18 \mathrm{~A})-\mathrm{C}(18)-\mathrm{H}(18 \mathrm{~B})$ & 109.5 \\
\hline $\mathrm{C}(15)-\mathrm{C}(18)-\mathrm{H}(18 \mathrm{C})$ & 109.5 & H(18A)-C(18)-H(18C) & 109.5 \\
\hline $\mathrm{H}(18 \mathrm{~B})-\mathrm{C}(18)-\mathrm{H}(18 \mathrm{C})$ & 109.5 & $\mathrm{C}(20)-\mathrm{C}(19)-\mathrm{C}(24)$ & $119.0(5)$ \\
\hline$C(20)-C(19)-C(10)$ & $120.0(5)$ & C(24)-C(19)-C(10) & $120.7(5)$ \\
\hline $\mathrm{C}(21)-\mathrm{C}(20)-\mathrm{C}(19)$ & $121.2(6)$ & $\mathrm{C}(21)-\mathrm{C}(20)-\mathrm{H}(20)$ & 119.4 \\
\hline $\mathrm{C}(19)-\mathrm{C}(20)-\mathrm{H}(20)$ & 119.4 & $\mathrm{C}(20)-\mathrm{C}(21)-\mathrm{C}(22)$ & 119.8(6) \\
\hline $\mathrm{C}(20)-\mathrm{C}(21)-\mathrm{H}(21)$ & 120.1 & $\mathrm{C}(22)-\mathrm{C}(21)-\mathrm{H}(21)$ & 120.1 \\
\hline $\mathrm{C}(23)-\mathrm{C}(22)-\mathrm{C}(21)$ & $119.4(6)$ & $\mathrm{C}(23)-\mathrm{C}(22)-\mathrm{H}(22)$ & 120.3 \\
\hline $\mathrm{C}(21)-\mathrm{C}(22)-\mathrm{H}(22)$ & 120.3 & $\mathrm{C}(22)-\mathrm{C}(23)-\mathrm{C}(24)$ & $120.7(5)$ \\
\hline $\mathrm{C}(22)-\mathrm{C}(23)-\mathrm{H}(23)$ & 119.6 & $\mathrm{C}(24)-\mathrm{C}(23)-\mathrm{H}(23)$ & 119.6 \\
\hline $\mathrm{C}(23)-\mathrm{C}(24)-\mathrm{C}(19)$ & 119.6(5) & $\mathrm{C}(23)-\mathrm{C}(24)-\mathrm{H}(24)$ & 120.2 \\
\hline $\mathrm{C}(19)-\mathrm{C}(24)-\mathrm{H}(24)$ & 120.2 & $\mathrm{C}(30)-\mathrm{C}(25)-\mathrm{C}(26)$ & $119.6(5)$ \\
\hline
\end{tabular}




$\begin{array}{llll}\mathrm{C}(30)-\mathrm{C}(25)-\mathrm{C}(11) & 121.2(4) & \mathrm{C}(26)-\mathrm{C}(25)-\mathrm{C}(11) & 119.0(5) \\ \mathrm{C}(27)-\mathrm{C}(26)-\mathrm{C}(25) & 119.8(5) & \mathrm{C}(27)-\mathrm{C}(26)-\mathrm{H}(26) & 120.1 \\ \mathrm{C}(25)-\mathrm{C}(26)-\mathrm{H}(26) & 120.1 & \mathrm{C}(28)-\mathrm{C}(27)-\mathrm{C}(26) & 120.4(5) \\ \mathrm{C}(28)-\mathrm{C}(27)-\mathrm{H}(27) & 119.8 & \mathrm{C}(26)-\mathrm{C}(27)-\mathrm{H}(27) & 119.8 \\ \mathrm{C}(27)-\mathrm{C}(28)-\mathrm{C}(29) & 120.3(5) & \mathrm{C}(27)-\mathrm{C}(28)-\mathrm{H}(28) & 119.9 \\ \mathrm{C}(29)-\mathrm{C}(28)-\mathrm{H}(28) & 119.9 & \mathrm{C}(28)-\mathrm{C}(29)-\mathrm{C}(30) & 119.9(6) \\ \mathrm{C}(28)-\mathrm{C}(29)-\mathrm{H}(29) & 120.0 & \mathrm{C}(30)-\mathrm{C}(29)-\mathrm{H}(29) & 120.0 \\ \mathrm{C}(25)-\mathrm{C}(30)-\mathrm{C}(29) & 120.0(5) & \mathrm{C}(25)-\mathrm{C}(30)-\mathrm{H}(30) & 120.0 \\ \mathrm{C}(29)-\mathrm{C}(30)-\mathrm{H}(30) & 120.0 & & \end{array}$

Symmetry transformations used to generate equivalent atoms: 
Table S13. Torsion angles [ $\left.{ }^{\circ}\right]$ for VGB-B-137(5).

\begin{tabular}{|c|c|c|c|}
\hline atom-atom-atom-atom & angle & atom-atom-atom-atom & angle \\
\hline $\mathrm{C}(1)-\mathrm{N}(1)-\mathrm{N}(2)-\mathrm{C}(3)$ & $1.4(6)$ & $\mathrm{C}(9)-\mathrm{N}(4)-\mathrm{N}(5)-\mathrm{C}(10)$ & $2.9(7)$ \\
\hline $\mathrm{N}(2)-\mathrm{N}(1)-\mathrm{C}(1)-\mathrm{C}(2)$ & $-0.9(6)$ & $\mathrm{N}(2)-\mathrm{N}(1)-\mathrm{C}(1)-\mathrm{C}(12)$ & $-177.0(4)$ \\
\hline N(1)-C(1)-C(2)-C(3) & $0.2(6)$ & $C(12)-C(1)-C(2)-C(3)$ & 175.5(5) \\
\hline N(1)-C(1)-C(2)-C(4) & 174.9(5) & $\mathrm{C}(12)-\mathrm{C}(1)-\mathrm{C}(2)-\mathrm{C}(4)$ & $-9.8(9)$ \\
\hline $\mathrm{N}(1)-\mathrm{N}(2)-\mathrm{C}(3)-\mathrm{C}(2)$ & $-1.3(6)$ & $\mathrm{C}(1)-\mathrm{C}(2)-\mathrm{C}(3)-\mathrm{N}(2)$ & $0.6(5)$ \\
\hline $\mathrm{C}(4)-\mathrm{C}(2)-\mathrm{C}(3)-\mathrm{N}(2)$ & $-174.8(5)$ & $\mathrm{C}(8)-\mathrm{N}(3)-\mathrm{C}(4)-\mathrm{C}(5)$ & $-1.0(7)$ \\
\hline $\mathrm{C}(8)-\mathrm{N}(3)-\mathrm{C}(4)-\mathrm{C}(2)$ & 175.2(4) & $\mathrm{C}(3)-\mathrm{C}(2)-\mathrm{C}(4)-\mathrm{N}(3)$ & $-20.3(7)$ \\
\hline $\mathrm{C}(1)-\mathrm{C}(2)-\mathrm{C}(4)-\mathrm{N}(3)$ & 165.7(5) & $C(3)-C(2)-C(4)-C(5)$ & 155.8(5) \\
\hline$C(1)-C(2)-C(4)-C(5)$ & $-18.1(8)$ & $\mathrm{N}(3)-\mathrm{C}(4)-\mathrm{C}(5)-\mathrm{C}(6)$ & $-0.2(7)$ \\
\hline$C(2)-C(4)-C(5)-C(6)$ & $-176.1(5)$ & $C(4)-C(5)-C(6)-C(7)$ & $1.0(7)$ \\
\hline$C(5)-C(6)-C(7)-C(8)$ & $-0.7(7)$ & $\mathrm{C}(4)-\mathrm{N}(3)-\mathrm{C}(8)-\mathrm{C}(7)$ & $1.3(7)$ \\
\hline $\mathrm{C}(4)-\mathrm{N}(3)-\mathrm{C}(8)-\mathrm{C}(9)$ & $-178.2(4)$ & $\mathrm{C}(6)-\mathrm{C}(7)-\mathrm{C}(8)-\mathrm{N}(3)$ & $-0.5(7)$ \\
\hline $\mathrm{C}(6)-\mathrm{C}(7)-\mathrm{C}(8)-\mathrm{C}(9)$ & 179.0(4) & $\mathrm{C}(11)-\mathrm{N}(6)-\mathrm{C}(9)-\mathrm{N}(4)$ & $6.7(7)$ \\
\hline C(11)-N(6)-C(9)-C(8) & $-175.9(4)$ & N(5)-N(4)-C(9)-N(6) & $-12.2(7)$ \\
\hline $\mathrm{N}(5)-\mathrm{N}(4)-\mathrm{C}(9)-\mathrm{C}(8)$ & $170.4(4)$ & $\mathrm{N}(3)-\mathrm{C}(8)-\mathrm{C}(9)-\mathrm{N}(6)$ & $174.5(4)$ \\
\hline $\mathrm{C}(7)-\mathrm{C}(8)-\mathrm{C}(9)-\mathrm{N}(6)$ & $-5.1(7)$ & $\mathrm{N}(3)-\mathrm{C}(8)-\mathrm{C}(9)-\mathrm{N}(4)$ & $-8.0(6)$ \\
\hline C(7)-C(8)-C(9)-N(4) & $172.4(5)$ & $\mathrm{N}(4)-\mathrm{N}(5)-\mathrm{C}(10)-\mathrm{C}(11)$ & $10.5(7)$ \\
\hline $\mathrm{N}(4)-\mathrm{N}(5)-\mathrm{C}(10)-\mathrm{C}(19)$ & $-169.0(4)$ & $\mathrm{C}(9)-\mathrm{N}(6)-\mathrm{C}(11)-\mathrm{C}(10)$ & $7.6(6)$ \\
\hline $\mathrm{C}(9)-\mathrm{N}(6)-\mathrm{C}(11)-\mathrm{C}(25)$ & $-170.4(4)$ & $\mathrm{N}(5)-\mathrm{C}(10)-\mathrm{C}(11)-\mathrm{N}(6)$ & $-16.0(7)$ \\
\hline $\mathrm{C}(19)-\mathrm{C}(10)-\mathrm{C}(11)-\mathrm{N}(6)$ & $163.4(5)$ & N(5)-C(10)-C(11)-C(25) & $161.8(5)$ \\
\hline $\mathrm{C}(19)-\mathrm{C}(10)-\mathrm{C}(11)-\mathrm{C}(25)$ & $-18.7(8)$ & $\mathrm{N}(1)-\mathrm{C}(1)-\mathrm{C}(12)-\mathrm{C}(17)$ & $-53.1(7)$ \\
\hline$C(2)-C(1)-C(12)-C(17)$ & 131.7(6) & $\mathrm{N}(1)-\mathrm{C}(1)-\mathrm{C}(12)-\mathrm{C}(13)$ & 124.3(5) \\
\hline$C(2)-C(1)-C(12)-C(13)$ & $-50.8(8)$ & $\mathrm{C}(17)-\mathrm{C}(12)-\mathrm{C}(13)-\mathrm{C}(14)$ & $0.7(7)$ \\
\hline $\mathrm{C}(1)-\mathrm{C}(12)-\mathrm{C}(13)-\mathrm{C}(14)$ & $-176.8(5)$ & $\mathrm{C}(12)-\mathrm{C}(13)-\mathrm{C}(14)-\mathrm{C}(15)$ & $-0.3(8)$ \\
\hline $\mathrm{C}(13)-\mathrm{C}(14)-\mathrm{C}(15)-\mathrm{C}(16)$ & $-0.1(8)$ & $C(13)-C(14)-C(15)-C(18)$ & $-179.4(5)$ \\
\hline $\mathrm{C}(14)-\mathrm{C}(15)-\mathrm{C}(16)-\mathrm{C}(17)$ & $0.2(9)$ & $\mathrm{C}(18)-\mathrm{C}(15)-\mathrm{C}(16)-\mathrm{C}(17)$ & 179.5(6) \\
\hline $\mathrm{C}(13)-\mathrm{C}(12)-\mathrm{C}(17)-\mathrm{C}(16)$ & $-0.7(8)$ & $C(1)-C(12)-C(17)-C(16)$ & 176.8(5) \\
\hline $\mathrm{C}(15)-\mathrm{C}(16)-\mathrm{C}(17)-\mathrm{C}(12)$ & $0.2(9)$ & N(5)-C(10)-C(19)-C(20) & $135.4(6)$ \\
\hline $\mathrm{C}(11)-\mathrm{C}(10)-\mathrm{C}(19)-\mathrm{C}(20)$ & $-44.1(8)$ & N(5)-C(10)-C(19)-C(24) & $-37.4(7)$ \\
\hline $\mathrm{C}(11)-\mathrm{C}(10)-\mathrm{C}(19)-\mathrm{C}(24)$ & 143.1(5) & $C(24)-C(19)-C(20)-C(21)$ & $-1.7(10)$ \\
\hline$C(10)-C(19)-C(20)-C(21)$ & $-174.6(7)$ & $\mathrm{C}(19)-\mathrm{C}(20)-\mathrm{C}(21)-\mathrm{C}(22)$ & $-2.0(13)$ \\
\hline $\mathrm{C}(20)-\mathrm{C}(21)-\mathrm{C}(22)-\mathrm{C}(23)$ & $3.0(12)$ & $C(21)-C(22)-C(23)-C(24)$ & $-0.3(10)$ \\
\hline $\mathrm{C}(22)-\mathrm{C}(23)-\mathrm{C}(24)-\mathrm{C}(19)$ & $-3.3(9)$ & $\mathrm{C}(20)-\mathrm{C}(19)-\mathrm{C}(24)-\mathrm{C}(23)$ & $4.3(8)$ \\
\hline $\mathrm{C}(10)-\mathrm{C}(19)-\mathrm{C}(24)-\mathrm{C}(23)$ & 177.1(5) & N(6)-C(11)-C(25)-C(30) & 139.1(5) \\
\hline $\mathrm{C}(10)-\mathrm{C}(11)-\mathrm{C}(25)-\mathrm{C}(30)$ & $-38.8(8)$ & N(6)-C(11)-C(25)-C(26) & $-36.1(7)$ \\
\hline $\mathrm{C}(10)-\mathrm{C}(11)-\mathrm{C}(25)-\mathrm{C}(26)$ & $146.0(5)$ & $C(30)-C(25)-C(26)-C(27)$ & $1.1(8)$ \\
\hline $\mathrm{C}(11)-\mathrm{C}(25)-\mathrm{C}(26)-\mathrm{C}(27)$ & 176.4(5) & $\mathrm{C}(25)-\mathrm{C}(26)-\mathrm{C}(27)-\mathrm{C}(28)$ & $-0.7(8)$ \\
\hline $\mathrm{C}(26)-\mathrm{C}(27)-\mathrm{C}(28)-\mathrm{C}(29)$ & $-0.3(9)$ & $C(27)-C(28)-C(29)-C(30)$ & $0.9(9)$ \\
\hline$C(26)-C(25)-C(30)-C(29)$ & $-0.5(8)$ & $\mathrm{C}(11)-\mathrm{C}(25)-\mathrm{C}(30)-\mathrm{C}(29)$ & $-175.7(5)$ \\
\hline $\mathrm{C}(28)-\mathrm{C}(29)-\mathrm{C}(30)-\mathrm{C}(25)$ & $-0.5(9)$ & & \\
\hline
\end{tabular}

Symmetry transformations used to generate equivalent atoms: 
Table S14. Hydrogen bonds for VGB-B-137(5) [Å and $\left.{ }^{\circ}\right]$.

D-H...A

$\mathrm{d}(\mathrm{D}-\mathrm{H})$

$\mathrm{d}(\mathrm{H} . . . \mathrm{A})$

d(D...A)

$<$ (DHA)

$\mathrm{N}(2)-\mathrm{H}(2 \mathrm{~N}) \ldots \mathrm{N}(4) \# 1$

$0.96(6)$

2.62(6)

$3.472(7)$

147(5)

$\mathrm{N}(2)-\mathrm{H}(2 \mathrm{~N}) \ldots \mathrm{N}(5) \# 1$

$0.96(6)$

2.09(6)

2.988(7)

153(5)

Symmetry transformations used to generate equivalent atoms:

$\# 1-x+2,-y+1,-z+1$ 


\section{Computational Data Supporting Information}

Table S15. Electronic Energies at B3LYP/DZVP2 Level.

*All structures are minima with zero imaginary frequencies.

\begin{tabular}{|c|c|c|c|}
\hline Molecule & $\Delta \mathbf{H}_{(\mathbf{O K})}$ & $\Delta \mathbf{H}_{(298 K)}$ & $\Delta \mathbf{G}_{(298 K)}$ \\
\hline 1 & -1065.560381 & -1065.539124 & -1065.611882 \\
\hline$p-\mathrm{Me}-\mathrm{C}_{6} \mathrm{H}_{4}-\mathrm{CH}=\mathrm{N}-\mathrm{NH}-\mathrm{Ts}$ & -1239.185087 & -1239.164575 & -1239.238310 \\
\hline 2 Major & -1484.751287 & -1484.721551 & -1484.816778 \\
\hline 2 Tautomer & -1484.738609 & -1484.708686 & -1484.805336 \\
\hline 3 Minor & -1484.73755 & -1484.70787 & -1484.80205 \\
\hline --Me- $\mathrm{C}_{6} \mathrm{H}_{4}-\mathrm{SO}_{2} \mathrm{H}$ & -820.092847 & -820.083247 & -820.127464 \\
\hline
\end{tabular}


Table S16. Bond Lengths ( $\AA$ ) for Structure 2.

\begin{tabular}{|c|c|c|}
\hline Atom - Atom & Calculated & Experimental \\
\hline$C(55)-C(52)$ & 1.506 & 1.513 \\
\hline$C(52)-C(50)$ & 1.392 & 1.402 \\
\hline$C(50)-C(47)$ & 1.386 & 1.399 \\
\hline$C(47)-C(45)$ & 1.393 & 1.404 \\
\hline$C(45)-C(46)$ & 1.399 & 1.408 \\
\hline$C(46)-C(48)$ & 1.387 & 1.394 \\
\hline$C(48)-C(52)$ & 1.393 & 1.407 \\
\hline$C(45)-C(14)$ & 1.472 & 1.474 \\
\hline $\mathrm{C}(14)-\mathrm{C}(13)$ & 1.409 & 1.421 \\
\hline$C(13)-C(12)$ & 1.384 & 1.391 \\
\hline $\mathrm{C}(12)-\mathrm{N}(20)$ & 1.355 & 1.363 \\
\hline $\mathrm{N}(20)-\mathrm{N}(22)$ & 1.351 & 1.343 \\
\hline $\mathrm{N}(22)-\mathrm{C}(14)$ & 1.342 & 1.349 \\
\hline $\mathrm{C}(12)-\mathrm{C}(5)$ & 1.461 & 1.465 \\
\hline$C(5)-C(4)$ & 1.396 & 1.406 \\
\hline$C(4)-C(3)$ & 1.383 & 1.394 \\
\hline$C(3)-C(2)$ & 1.385 & 1.398 \\
\hline$C(2)-C(1)$ & 1.392 & 1.403 \\
\hline $\mathrm{C}(1)-\mathrm{N}(19)$ & 1.340 & 1.339 \\
\hline $\mathrm{N}(19)-\mathrm{C}(5)$ & 1.344 & 1.343 \\
\hline $\mathrm{C}(1)-\mathrm{C}(9)$ & 1.489 & 1.494 \\
\hline $\mathrm{C}(9)-\mathrm{N}(16)$ & 1.347 & 1.341 \\
\hline $\mathrm{N}(16)-\mathrm{C}(10)$ & 1.325 & 1.335 \\
\hline $\mathrm{C}(10)-\mathrm{C}(11)$ & 1.422 & 1.426 \\
\hline $\mathrm{C}(11)-\mathrm{N}(18)$ & 1.339 & 1.346 \\
\hline $\mathrm{N}(18)-\mathrm{N}(17)$ & 1.342 & 1.326 \\
\hline $\mathrm{N}(17)-\mathrm{C}(9)$ & 1.336 & 1.347 \\
\hline$C(10)-C(23)$ & 1.487 & 1.483 \\
\hline$C(23)-C(25)$ & 1.391 & 1.406 \\
\hline$C(25)-C(28)$ & 1.391 & 1.398 \\
\hline$C(28)-C(30)$ & 1.383 & 1.401 \\
\hline$C(30)-C(26)$ & 1.382 & 1.401 \\
\hline$C(26)-C(24)$ & 1.388 & 1.397 \\
\hline$C(24)-C(23)$ & 1.395 & 1.407 \\
\hline$C(11)-C(34)$ & 1.484 & 1.485 \\
\hline$C(34)-C(36)$ & 1.395 & 1.407 \\
\hline$C(36)-C(39)$ & 1.390 & 1.397 \\
\hline $\mathrm{C}(39)-\mathrm{C}(41)$ & 1.388 & 1.401 \\
\hline$C(41)-C(37)$ & 1.382 & 1.401 \\
\hline $\mathrm{C}(37)-\mathrm{C}(35)$ & 1.389 & 1.398 \\
\hline$C(35)-C(34)$ & 1.397 & 1.407 \\
\hline
\end{tabular}




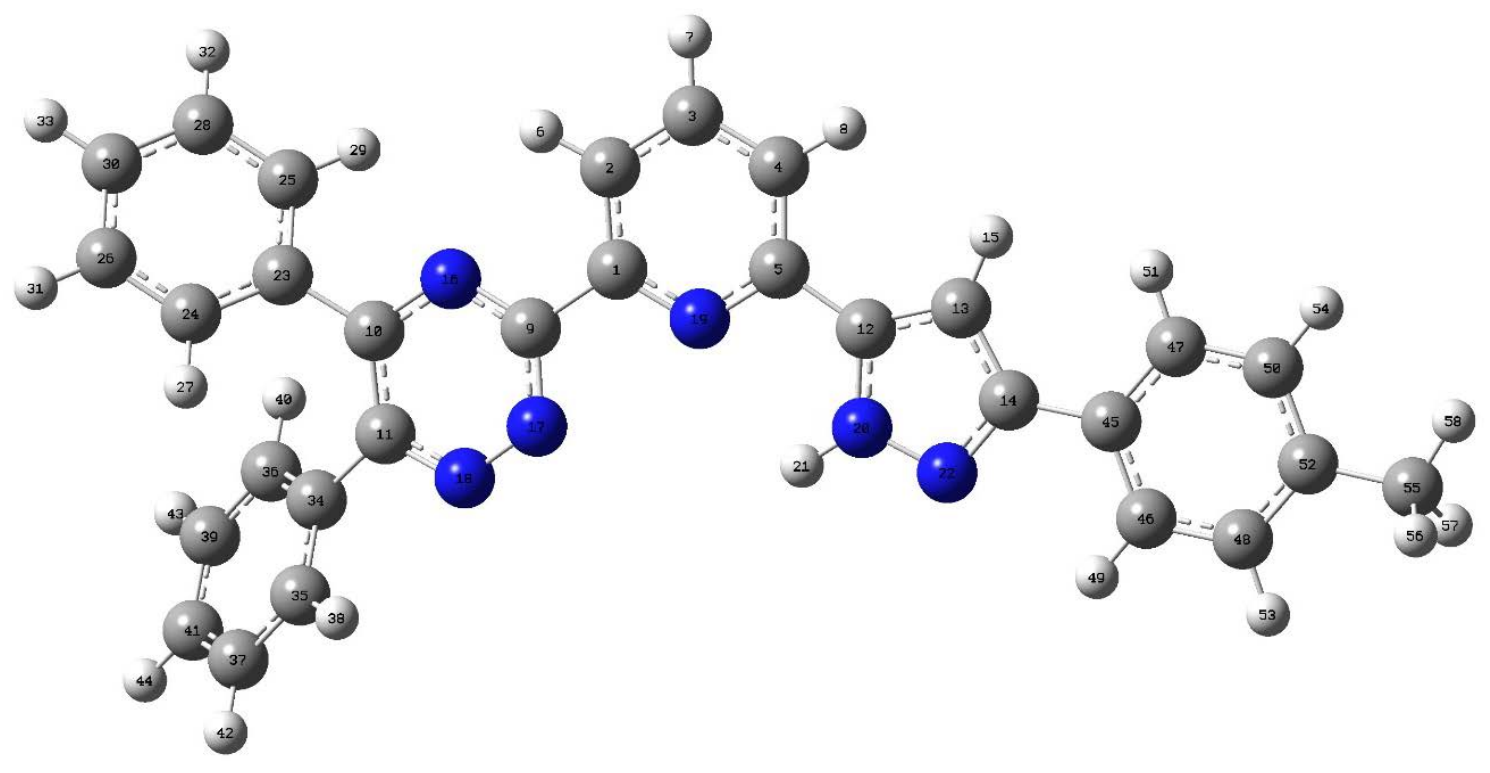


Table S17. XYZ B3LYP/DZVP2 optimized

\section{geometries 1}

01

$\begin{array}{llll}\text { C } & 5.086215 & -0.335829 & 0.106672\end{array}$

$\begin{array}{llll}\text { C } & 2.880404 & 0.280682 & -0.105084\end{array}$

$\begin{array}{llll}\text { C } & 3.209932 & 1.646390 & -0.127732\end{array}$

$\begin{array}{llll}\text { C } & 4.555039 & 2.008963 & -0.031281\end{array}$

$\begin{array}{llll}\mathrm{H} & 2.423855 & 2.384663 & -0.223928\end{array}$

$\begin{array}{llll}\mathrm{H} & 4.849169 & 3.054346 & -0.051302\end{array}$

$\begin{array}{llll}\text { C } & 6.059753 & -1.392717 & 0.238765\end{array}$

$\begin{array}{llll}\text { C } & 6.900877 & -2.261424 & 0.351272\end{array}$

H $\quad \begin{array}{llll}7.626189 & -3.038392 & 0.448423\end{array}$

$\begin{array}{llll}\text { C } & 1.450243 & -0.140026 & -0.212069\end{array}$

$\begin{array}{llll}\text { C } & -0.766942 & 0.431967 & -0.086647\end{array}$

$\begin{array}{llll}C & -1.062538 & -0.959071 & -0.174389\end{array}$

$\begin{array}{llll}\mathrm{N} & -0.071537 & -1.821716 & -0.470371\end{array}$

N $\quad \begin{array}{llll}1.190041 & -1.421591 & -0.534574\end{array}$

$\begin{array}{llll}\mathrm{N} & 0.514439 & 0.805608 & -0.044104\end{array}$

$\begin{array}{llll}\text { C } & -2.393583 & -1.579895 & 0.045543\end{array}$

$\begin{array}{llll}\text { C } & -2.779007 & -2.691341 & -0.726057\end{array}$

$\begin{array}{llll}\text { C } & -3.251717 & -1.122911 & 1.062767\end{array}$

$\begin{array}{llll}\text { C } & -4.010038 & -3.314748 & -0.503073\end{array}$

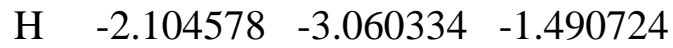

$\begin{array}{llll}\text { C } & -4.475534 & -1.757090 & 1.292854\end{array}$

$\begin{array}{llll}\mathrm{H} & -2.960081 & -0.281257 & 1.681581\end{array}$

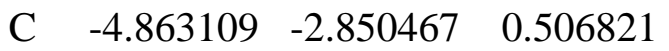

H $\quad-4.301240 \quad-4.166118-1.111286$

$\begin{array}{llll}\mathrm{H} & -5.125072 & -1.402000 & 2.087492\end{array}$

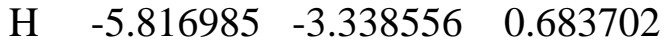

$\begin{array}{llll}\text { C } & -1.772261 & 1.527549 & -0.092437\end{array}$

$\begin{array}{llll}\text { C } & -2.927140 & 1.459924 & -0.893019\end{array}$

$\begin{array}{llll}C & -1.530316 & 2.688389 & 0.664025\end{array}$

$\begin{array}{llll}\text { C } & -3.824472 & 2.530525 & -0.927426\end{array}$

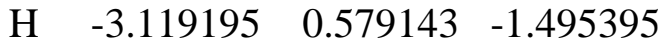

$\begin{array}{llll}\text { C } & -2.439682 & 3.749537 & 0.643856\end{array}$

$\begin{array}{llll}\mathrm{H} & -0.629846 & 2.746561 & 1.265748\end{array}$

$\begin{array}{llll}\text { C } & -3.589209 & 3.675130 & -0.153952\end{array}$

$\begin{array}{llll}\mathrm{H} & -4.706661 & 2.473189 & -1.558048\end{array}$

$\begin{array}{llll}\mathrm{H} & -2.251325 & 4.634795 & 1.244159\end{array}$

H $\quad-4.293287 \quad 4.501691 \quad-0.175414$

$\begin{array}{llll}\mathrm{N} & 3.794914 & -0.693131 & 0.009953\end{array}$

$\begin{array}{llll}\text { C } & 5.516538 & 1.005409 & 0.087621\end{array}$

H $\quad 6.5733521 .234389 \quad 0.166476$ 


$\begin{array}{cccc}\boldsymbol{p}-\mathbf{M e}-\mathbf{C}_{6} \mathbf{H}_{\mathbf{4}-\mathbf{C H}} \mathbf{N}-\mathbf{N H}-\mathbf{T s} & \\ \text { 0 1 } & & & \\ \mathrm{C} & 3.001046 & -2.030841 & 0.806999 \\ \mathrm{C} & 2.367655 & -0.805232 & 1.041123 \\ \mathrm{C} & 2.623264 & 0.259761 & 0.175810 \\ \mathrm{C} & 3.504387 & 0.129478 & -0.903721 \\ \mathrm{C} & 4.126633 & -1.100644 & -1.119255 \\ \mathrm{C} & 3.885743 & -2.197536 & -0.269911 \\ \mathrm{H} & 2.807611 & -2.865135 & 1.475255 \\ \mathrm{H} & 1.693081 & -0.671154 & 1.879302 \\ \mathrm{H} & 3.702635 & 0.977528 & -1.550368 \\ \mathrm{H} & 4.814499 & -1.210400 & -1.953389 \\ \mathrm{C} & 4.584376 & -3.517547 & -0.509898 \\ \mathrm{H} & 4.488716 & -3.829152 & -1.554768 \\ \mathrm{H} & 5.654898 & -3.435704 & -0.290227 \\ \mathrm{H} & 4.171289 & -4.307351 & 0.121858 \\ \mathrm{~S} & 1.832903 & 1.836392 & 0.472082 \\ \mathrm{O} & 2.660970 & 2.924513 & -0.065560 \\ \mathrm{O} & 1.350042 & 1.857858 & 1.855708 \\ \mathrm{~N} & 0.516858 & 1.793858 & -0.646711 \\ \mathrm{H} & 0.287550 & 2.759831 & -0.906696 \\ \mathrm{~N} & -0.531384 & 0.980907 & -0.262606 \\ \mathrm{C} & -1.702469 & 1.294908 & -0.691743 \\ \mathrm{H} & -1.861559 & 2.196685 & -1.297161 \\ \mathrm{C} & -2.888528 & 0.477262 & -0.411665 \\ \mathrm{C} & -2.821084 & -0.710031 & 0.347190 \\ \mathrm{C} & -4.137480 & 0.886578 & -0.907816 \\ \mathrm{C} & -3.972277 & -1.452699 & 0.593307 \\ \mathrm{H} & -1.860147 & -1.031488 & 0.734294 \\ \mathrm{C} & -5.290113 & 0.133280 & -0.655720 \\ \mathrm{H} & -4.209829 & 1.799123 & -1.494565 \\ \mathrm{C} & -5.228761 & -1.047031 & 0.097701 \\ \mathrm{H} & -3.902769 & -2.364865 & 1.181056 \\ \mathrm{H} & -6.245726 & 0.468996 & -1.049137 \\ \mathrm{C} & -6.467432 & -1.868449 & 0.378982 \\ \mathrm{H} & -7.352263 & -1.425181 & -0.084436 \\ \mathrm{H} & -6.358760 & -2.888792 & -0.004861 \\ \mathrm{H} & -6.651840 & -1.945075 & 1.456278\end{array}$

2 major

01
C $\quad-0.315749 \quad-1.426749 \quad-0.078756$
$\begin{array}{llll}\text { C } & -0.432660 & -2.822104 & 0.012401\end{array}$
$\begin{array}{llll}\text { C } & 0.738944 & -3.580121 & 0.100642\end{array}$
C $\quad 1.973281-2.931443 \quad 0.093958$
$\begin{array}{llll}\text { C } & 1.989600 & -1.528460 & -0.001535\end{array}$ 


\begin{tabular}{|c|c|c|c|}
\hline & 13196 & -3.280275 & \\
\hline & 0.688483 & -4.662878 & 959 \\
\hline & 2.900315 & -3.490010 & \\
\hline & -1.538826 & -0.573784 & \\
\hline & -3.823698 & -0.413670 & -0 \\
\hline & -3.663036 & 1.001569 & -0 \\
\hline & 3.234988 & -0.756429 & -0.017717 \\
\hline & 4.582365 & -1.092075 & 0.0 \\
\hline & 5.281896 & 0.1 & \\
\hline & 4.996896 & -2.083579 & 0 . \\
\hline & -2.728418 & -1.1 & \\
\hline & -1.374804 & 0.732885 & -0 . \\
\hline & -2.4 & 1.5 & \\
\hline & 0.865865 & -0.797944 & 5883 \\
\hline & 3.1 & 0.6 & \\
\hline & 2.35 & 1.1 & -0 . \\
\hline & 4.4 & 1.1 & -0 . \\
\hline & -5.1 & -1.1 & \\
\hline & -6.188012 & -0.692088 & -0. \\
\hline & -5.273 & -2.3 & \\
\hline & -7.377874 & -1.4 & -0 \\
\hline & -6.08 & 0.2 & -1 . \\
\hline C & -6.472 & -3.0 & 26 \\
\hline & -4.4 & -2.6 & \\
\hline C & -7.527 & -2.5 & -0 . \\
\hline & -8.187827 & -1.08 & -1 \\
\hline $\mathrm{H}$ & -6.582 & -3.9 & 357 \\
\hline & -8.457407 & -3.1 & -0.251064 \\
\hline $\mathrm{C}$ & -4.73 & 2.0 & 194 \\
\hline & -4.737451 & 3.192 & -0.672274 \\
\hline $\mathrm{C}$ & -5.708322 & 1.83 & 1.085366 \\
\hline $\mathrm{C}$ & -5.712228 & 4.170 & -0.452475 \\
\hline $\mathrm{H}$ & -3.969988 & 3.33 & -1.424107 \\
\hline $\mathrm{C}$ & -6.672759 & 2.8 & 753 \\
\hline $\mathrm{H}$ & -5.7071 & 0.9 & 1.693067 \\
\hline C & -6.683393 & 3.98 & 0.540615 \\
\hline & -5.710822 & 5.077 & -1.049983 \\
\hline $\mathrm{H}$ & -7.413333 & 2.675879 & 2.094344 \\
\hline $\mathrm{H}$ & -7.437183 & 4.748772 & 0.715197 \\
\hline C & 6.736659 & 0.379723 & 0.015740 \\
\hline C & 7.253206 & 1.687353 & -0.066103 \\
\hline U & 7.647650 & -0.682797 & 0.128842 \\
\hline C & 8.628669 & 1.914903 & -0.035464 \\
\hline F & 6.561419 & 2.517948 & -0.153590 \\
\hline C & 9.026940 & -0.447824 & 0.159178 \\
\hline & 7.286169 & -1.704493 & 0.19399 \\
\hline
\end{tabular}




$\begin{array}{cccc}\mathrm{C} & 9.544777 & 0.852589 & 0.077582 \\ \mathrm{H} & 9.000386 & 2.935003 & -0.100214 \\ \mathrm{H} & 9.708560 & -1.289971 & 0.247509 \\ \mathrm{C} & 11.034982 & 1.113808 & 0.107355 \\ \mathrm{H} & 11.369702 & 1.600586 & -0.815640 \\ \mathrm{H} & 11.304516 & 1.774611 & 0.938760 \\ \mathrm{H} & 11.598445 & 0.183911 & 0.220123\end{array}$

\section{2 major(tautomer)}

01

$\begin{array}{llll}\text { C } & -0.320485 & -1.277430 & -0.253061\end{array}$

C $\quad-0.369334-2.680782 \quad-0.233595$

$\begin{array}{llll}\text { C } & 0.838107 & -3.377038 & -0.155622\end{array}$

$\begin{array}{llll}\text { C } & 2.030756 & -2.656416 & -0.093286\end{array}$

C $\quad \begin{array}{llll}\text { C } & 1.979016 & -1.249222 & -0.110435\end{array}$

$\mathrm{H} \quad-1.324090 \quad-3.188739 \quad-0.283468$

H $\quad 0.849825 \quad-4.463175 \quad-0.143361$

H $\quad 2.981223 \quad-3.172864 \quad-0.022521$

$\begin{array}{llll}\text { C } & -1.591509 & -0.496586 & -0.346513\end{array}$

$\begin{array}{llll}\text { C } & -3.869038 & -0.432871 & -0.078157\end{array}$

$\begin{array}{llll}\text { C } & -3.788377 & 0.974006 & -0.281395\end{array}$

$\begin{array}{llll}\text { C } & 3.225089 & -0.455557 & -0.030876\end{array}$

C $\quad 4.566820 \quad-0.929300 \quad-0.054560$

$\begin{array}{llll}\text { C } & 5.366986 & 0.200193 & 0.061877\end{array}$

$\mathrm{H} \quad 4.915334 \quad-1.948538 \quad-0.117790$

$\mathrm{N} \quad-2.733796 \quad-1.135916-0.055355$

$\begin{array}{llll}\mathrm{N} & -1.523416 & 0.777686 & -0.781568\end{array}$

$\begin{array}{llll}\mathrm{N} & -2.625600 & 1.508020 & -0.702578\end{array}$

$\begin{array}{llll}\mathrm{N} & 0.819713 & -0.579287 & -0.192918\end{array}$

$\begin{array}{llll}\mathrm{N} & 3.191599 & 0.881606 & 0.086164\end{array}$

N $\quad 4.486724 \quad 1.2476390 .150035$

C $\quad-5.128133 \quad-1.212707 \quad 0.059252$

$\begin{array}{llll}\text { C } & -6.276149 & -0.893703 & -0.688734\end{array}$

$\begin{array}{llll}\text { C } & -5.152216 & -2.337649 & 0.903213\end{array}$

C $\quad-7.426359-1.680587 \quad-0.585601$

$\mathrm{H} \quad-6.268156 \quad-0.040246 \quad-1.357367$

C $\quad-6.310163 \quad-3.111681 \quad 1.019837$

H $\quad-4.259417 \quad-2.593896 \quad 1.463126$

$\begin{array}{llll}\text { C } & -7.451179 & -2.786946 & 0.274121\end{array}$

H $\quad-8.302996 \quad-1.432301-1.176532$

$\mathrm{H} \quad-6.322519 \quad-3.969244 \quad 1.686207$

$\begin{array}{llll}\mathrm{H} & -8.349474 & -3.391346 & 0.359197\end{array}$

$\begin{array}{llll}\text { C } & -4.887110 & 1.949256 & -0.062387\end{array}$

$\begin{array}{llll}\text { C } & -5.014482 & 3.058358 & -0.918468\end{array}$

$\begin{array}{llll}\text { C } & -5.764516 & 1.825797 & 1.030641\end{array}$

C $\quad-6.016414 \quad 4.008661 \quad-0.701653$ 


$\begin{array}{cccc}\mathrm{H} & -4.319613 & 3.167761 & -1.743644 \\ \mathrm{C} & -6.756713 & 2.784564 & 1.252992 \\ \mathrm{H} & -5.666026 & 0.988571 & 1.713115 \\ \mathrm{C} & -6.891674 & 3.875851 & 0.384415 \\ \mathrm{H} & -6.110792 & 4.855805 & -1.374792 \\ \mathrm{H} & -7.421780 & 2.683475 & 2.105641 \\ \mathrm{H} & -7.666672 & 4.617092 & 0.555867 \\ \mathrm{C} & 6.825098 & 0.366686 & 0.097593 \\ \mathrm{C} & 7.428068 & 1.450667 & 0.759602 \\ \mathrm{C} & 7.664936 & -0.568307 & -0.538948 \\ \mathrm{C} & 8.819338 & 1.598429 & 0.776187 \\ \mathrm{H} & 6.816103 & 2.170850 & 1.295683 \\ \mathrm{C} & 9.051193 & -0.418909 & -0.511262 \\ \mathrm{H} & 7.224375 & -1.403592 & -1.074364 \\ \mathrm{C} & 9.657505 & 0.668910 & 0.143185 \\ \mathrm{H} & 9.257365 & 2.443509 & 1.300586 \\ \mathrm{H} & 9.675058 & -1.153504 & -1.014661 \\ \mathrm{C} & 11.162440 & 0.823759 & 0.157154 \\ \mathrm{H} & 11.555809 & 0.941892 & -0.858659 \\ \mathrm{H} & 11.465678 & 1.697895 & 0.738671 \\ \mathrm{H} & 11.646511 & -0.056987 & 0.592823 \\ \mathrm{H} & 4.708694 & 2.232668 & 0.176689\end{array}$

\section{3}

01

$\begin{array}{rrrr}\mathrm{C} & -0.783152 & 1.921143 & 0.051896 \\ \mathrm{C} & -0.621997 & 3.186466 & 0.631694 \\ \mathrm{C} & -1.770191 & 3.945288 & 0.876247 \\ \mathrm{C} & -3.014735 & 3.421993 & 0.530978 \\ \mathrm{C} & -3.081586 & 2.148198 & -0.076992 \\ \mathrm{H} & 0.368926 & 3.545525 & 0.877898 \\ \mathrm{H} & -1.694714 & 4.924723 & 1.340274 \\ \mathrm{H} & -3.924947 & 3.970871 & 0.746325 \\ \mathrm{C} & 0.408424 & 1.053984 & -0.196800 \\ \mathrm{C} & 2.677078 & 0.754038 & -0.040747 \\ \mathrm{C} & 2.464360 & -0.595592 & -0.446108 \\ \mathrm{C} & -4.390028 & 1.611311 & -0.505547 \\ \mathrm{C} & -5.401204 & 2.412788 & -1.026226 \\ \mathrm{H} & -5.415301 & 3.465528 & -1.269335 \\ \mathrm{~N} & 1.613784 & 1.540810 & 0.136700 \\ \mathrm{~N} & 0.206216 & -0.144493 & -0.777481 \\ \mathrm{~N} & 1.238795 & -0.971838 & -0.856442 \\ \mathrm{~N} & -1.977129 & 1.423727 & -0.302898 \\ \mathrm{~N} & -6.231843 & 0.318186 & -0.910388 \\ \mathrm{C} & 4.006616 & 1.390395 & 0.158731 \\ \mathrm{C} & 5.089668 & 1.109642 & -0.693923\end{array}$




$\begin{array}{lrrr}\mathrm{C} & 4.166807 & 2.346639 & 1.177402 \\ \mathrm{C} & 6.310055 & 1.768677 & -0.523348 \\ \mathrm{H} & 4.977029 & 0.387590 & -1.494851 \\ \mathrm{C} & 5.394685 & 2.988873 & 1.359653 \\ \mathrm{H} & 3.325102 & 2.576082 & 1.821741 \\ \mathrm{C} & 6.470206 & 2.703729 & 0.507994 \\ \mathrm{H} & 7.136029 & 1.554018 & -1.194842 \\ \mathrm{H} & 5.512400 & 3.714037 & 2.159509 \\ \mathrm{H} & 7.422720 & 3.207267 & 0.644177 \\ \mathrm{C} & 3.485166 & -1.674886 & -0.444557 \\ \mathrm{C} & 3.477723 & -2.638423 & -1.469573 \\ \mathrm{C} & 4.418333 & -1.796968 & 0.601416 \\ \mathrm{C} & 4.402700 & -3.686470 & -1.462812 \\ \mathrm{H} & 2.740757 & -2.559378 & -2.261084 \\ \mathrm{C} & 5.332619 & -2.853851 & 0.613210 \\ \mathrm{H} & 4.423880 & -1.075198 & 1.410946 \\ \mathrm{C} & 5.333629 & -3.798556 & -0.421736 \\ \mathrm{H} & 4.393640 & -4.418827 & -2.264759 \\ \mathrm{H} & 6.041499 & -2.943396 & 1.431076 \\ \mathrm{H} & 6.048690 & -4.615865 & -0.413565 \\ \mathrm{~N} & -6.466419 & 1.605542 & -1.250782 \\ \mathrm{C} & -4.967759 & 0.289786 & -0.454616 \\ \mathrm{H} & -7.352613 & 1.847557 & -1.669046 \\ \mathrm{C} & -4.452462 & -1.000532 & 0.061747 \\ \mathrm{C} & -3.126027 & -1.424727 & -0.117314 \\ \mathrm{C} & -5.349043 & -1.870530 & 0.715649 \\ \mathrm{C} & -2.714524 & -2.679058 & 0.350613 \\ \mathrm{H} & -2.412395 & -0.782728 & -0.617524 \\ \mathrm{C} & -4.927590 & -3.115171 & 1.182422 \\ \mathrm{H} & -6.379172 & -1.558988 & 0.851920 \\ \mathrm{C} & -3.598990 & -3.544665 & 1.008064 \\ \mathrm{H} & -1.683189 & -2.982438 & 0.190278 \\ \mathrm{H} & -5.638833 & -3.763823 & 1.689063 \\ \mathrm{C} & -3.149700 & -4.897268 & 1.517868 \\ \mathrm{H} & -2.102437 & -5.085560 & 1.267735 \\ \mathrm{H} & -3.253705 & -4.964195 & 2.607000 \\ \mathrm{H} & -3.751689 & -5.703850 & 1.084404\end{array}$

\section{$\mathrm{C}_{5} \mathrm{NH}_{4}-\mathrm{CCH}$ (A)}

01
$\begin{array}{llll}\text { C } & 0.000000 & 0.556056 & 0.000000\end{array}$
C $\quad-1.200698 \quad-0.179776 \quad 0.000000$
C $\quad-1.132456-1.574342 \quad 0.000000$
$\begin{array}{llll}\text { C } & 0.127454 & -2.183593 & 0.000000\end{array}$
C $\quad 1.262215 \quad-1.362114 \quad 0.000000$
$\mathrm{H} \quad-2.151480 \quad 0.341486 \quad 0.000000$ 


$\begin{array}{rrrr}\mathrm{H} & -2.039953 & -2.171040 & 0.000000 \\ \mathrm{H} & 0.232391 & -3.263820 & 0.000000 \\ \mathrm{H} & 2.257410 & -1.803229 & 0.000000 \\ \mathrm{~N} & 1.218487 & -0.024623 & 0.000000 \\ \mathrm{C} & -0.047564 & 1.998096 & 0.000000 \\ \mathrm{C} & -0.119233 & 3.210940 & 0.000000 \\ \mathrm{H} & -0.166081 & 4.277357 & 0.000000\end{array}$

\begin{tabular}{crrr}
\multicolumn{5}{c}{$\boldsymbol{p}-\mathbf{M e}-\mathbf{C}_{6} \mathbf{H}_{5}-\mathbf{C C H}$} \\
0 1 & & & \\
$\mathrm{C}$ & 1.109687 & 0.001102 & 0.000107 \\
$\mathrm{C}$ & 0.388583 & -1.211180 & -0.000803 \\
$\mathrm{C}$ & -1.006290 & -1.202126 & -0.002435 \\
$\mathrm{C}$ & -1.728912 & 0.004796 & -0.002648 \\
$\mathrm{C}$ & -1.004837 & 1.207724 & -0.002425 \\
$\mathrm{H}$ & 0.931042 & -2.150978 & -0.001344 \\
$\mathrm{H}$ & -1.544045 & -2.147216 & -0.004246 \\
$\mathrm{H}$ & -1.540838 & 2.152968 & -0.004131 \\
$\mathrm{C}$ & 2.545657 & -0.001268 & 0.000990 \\
$\mathrm{C}$ & 3.761997 & -0.003990 & 0.002017 \\
$\mathrm{H}$ & 4.829028 & -0.007273 & 0.003070 \\
$\mathrm{C}$ & 0.392720 & 1.213309 & -0.000712 \\
$\mathrm{C}$ & -3.242249 & -0.003055 & 0.003743 \\
$\mathrm{H}$ & -3.629291 & -0.426558 & 0.937471 \\
$\mathrm{H}$ & -3.643914 & 1.007570 & -0.100126 \\
$\mathrm{H}$ & -3.637712 & -0.612029 & -0.816533 \\
$\mathrm{H}$ & 0.937590 & 2.151637 & -0.001164
\end{tabular}

\section{Product (A)}

01

$\begin{array}{lrrr}\text { C } & -5.168736 & -0.749004 & 0.000047 \\ \mathrm{C} & -5.728849 & 0.533763 & 0.000020 \\ \mathrm{C} & -4.859920 & 1.632166 & -0.000027 \\ \mathrm{C} & -3.483702 & 1.403415 & -0.000041 \\ \mathrm{C} & -3.017403 & 0.077501 & -0.000002 \\ \mathrm{H} & -6.806002 & 0.663402 & 0.000034 \\ \mathrm{H} & -5.248558 & 2.646328 & -0.000055 \\ \mathrm{H} & -2.782065 & 2.230289 & -0.000086 \\ \mathrm{C} & -1.587476 & -0.243681 & -0.000006 \\ \mathrm{C} & -0.427730 & 0.524145 & 0.000005 \\ \mathrm{C} & 0.643596 & -0.409602 & -0.000010 \\ \mathrm{H} & -0.366265 & 1.601292 & 0.000046 \\ \mathrm{~N} & -3.852437 & -0.983897 & 0.000038 \\ \mathrm{~N} & -1.168469 & -1.541218 & -0.000036 \\ \mathrm{H} & -1.777564 & -2.348732 & -0.000036 \\ \mathrm{~N} & 0.169507 & -1.672065 & -0.000036\end{array}$




$\begin{array}{lccc}\mathrm{H} & -5.811646 & -1.627118 & 0.000079 \\ \mathrm{C} & 2.094308 & -0.145937 & -0.000003 \\ \mathrm{C} & 3.017483 & -1.209454 & -0.000005 \\ \mathrm{C} & 2.598464 & 1.164551 & 0.000011 \\ \mathrm{C} & 4.390100 & -0.963400 & 0.000005 \\ \mathrm{H} & 2.642694 & -2.227132 & -0.000008 \\ \mathrm{C} & 3.977158 & 1.404956 & 0.000022 \\ \mathrm{H} & 1.916739 & 2.009531 & 0.000020 \\ \mathrm{C} & 4.899154 & 0.348610 & 0.000015 \\ \mathrm{H} & 5.080973 & -1.803403 & 0.000004 \\ \mathrm{H} & 4.338802 & 2.430014 & 0.000036 \\ \mathrm{C} & 6.391209 & 0.601351 & 0.000003 \\ \mathrm{H} & 6.867419 & 0.158528 & 0.881931 \\ \mathrm{H} & 6.867310 & 0.159088 & -0.882267 \\ \mathrm{H} & 6.612021 & 1.671892 & 0.000324\end{array}$

